\title{
Next Generation Solvent Development for Caustic-Side Solvent Extraction of Cesium
}

\section{June 2013}

Prepared by

Bruce A. Moyer, Joseph F. Birdwell, Jr., Lætitia H. Delmau, Nathan C. Duncan, Dale D. Ensor, Talon G. Hill, Denise L. Lee, Benjamin D. Roach, Frederick V. Sloop, Jr., Erica L. Stoner, and Neil J. Williams 


\section{DOCUMENT AVAILABILITY}

Reports produced after January 1, 1996, are generally available free via the U.S. Department of Energy (DOE) Information Bridge.

Web site http://www.osti.gov/bridge

Reports produced before January 1, 1996, may be purchased by members of the public from the following source.

National Technical Information Service

5285 Port Royal Road

Springfield, VA 22161

Telephone 703-605-6000 (1-800-553-6847)

TDD 703-487-4639

Fax 703-605-6900

E-mail info@ntis.gov

Web site http://www.ntis.gov/support/ordernowabout.htm

Reports are available to DOE employees, DOE contractors, Energy Technology Data Exchange (ETDE) representatives, and International Nuclear Information System (INIS) representatives from the following source.

Office of Scientific and Technical Information

P.O. Box 62

Oak Ridge, TN 37831

Telephone 865-576-8401

Fax 865-576-5728

E-mail reports@osti.gov

Web site http://www.osti.gov/contact.html

This report was prepared as an account of work sponsored by an agency of the United States Government. Neither the United States Government nor any agency thereof, nor any of their employees, makes any warranty, express or implied, or assumes any legal liability or responsibility for the accuracy, completeness, or usefulness of any information, apparatus, product, or process disclosed, or represents that its use would not infringe privately owned rights. Reference herein to any specific commercial product, process, or service by trade name, trademark, manufacturer, or otherwise, does not necessarily constitute or imply its endorsement, recommendation, or favoring by the United States Government or any agency thereof. The views and opinions of authors expressed herein do not necessarily state or reflect those of the United States Government or any agency thereof. 
ORNL/TM-2013/224 REV1

Next-Generation CSSX Program

\title{
NEXT-GENERATION SOLVENT DEVELOPMENT FOR CAUSTIC- SIDE SOLVENT EXTRACTION OF CESIUM
}

\author{
Bruce A. Moyer, Joseph F. Birdwell, Jr., Lætitia H. Delmau, Nathan C. Duncan, \\ Dale D. Ensor, Talon G. Hill, Denise L. Lee, Benjamin D. Roach, \\ Frederick V. Sloop, Jr., Erica L. Stoner, and Neil J. Williams
}

Date Published: June 2013

This report was sponsored by and prepared for the U.S. Department of Energy, Savannah River Site. Revision 0 will undergo external review, possibly resulting in revisions to be issued as Revision 1 . The final revision will be issued for public release within one year. Consequently, all versions prior to public release should be treated as limited distribution for internal use by sponsors and program participants.

Prepared by

OAK RIDGE NATIONAL LABORATORY

Oak Ridge, Tennessee 37831-6283

managed by

UT-BATTELLE, LLC

for the

U.S. DEPARTMENT OF ENERGY

under contract DE-AC05-00OR22725 



\section{CONTENTS}

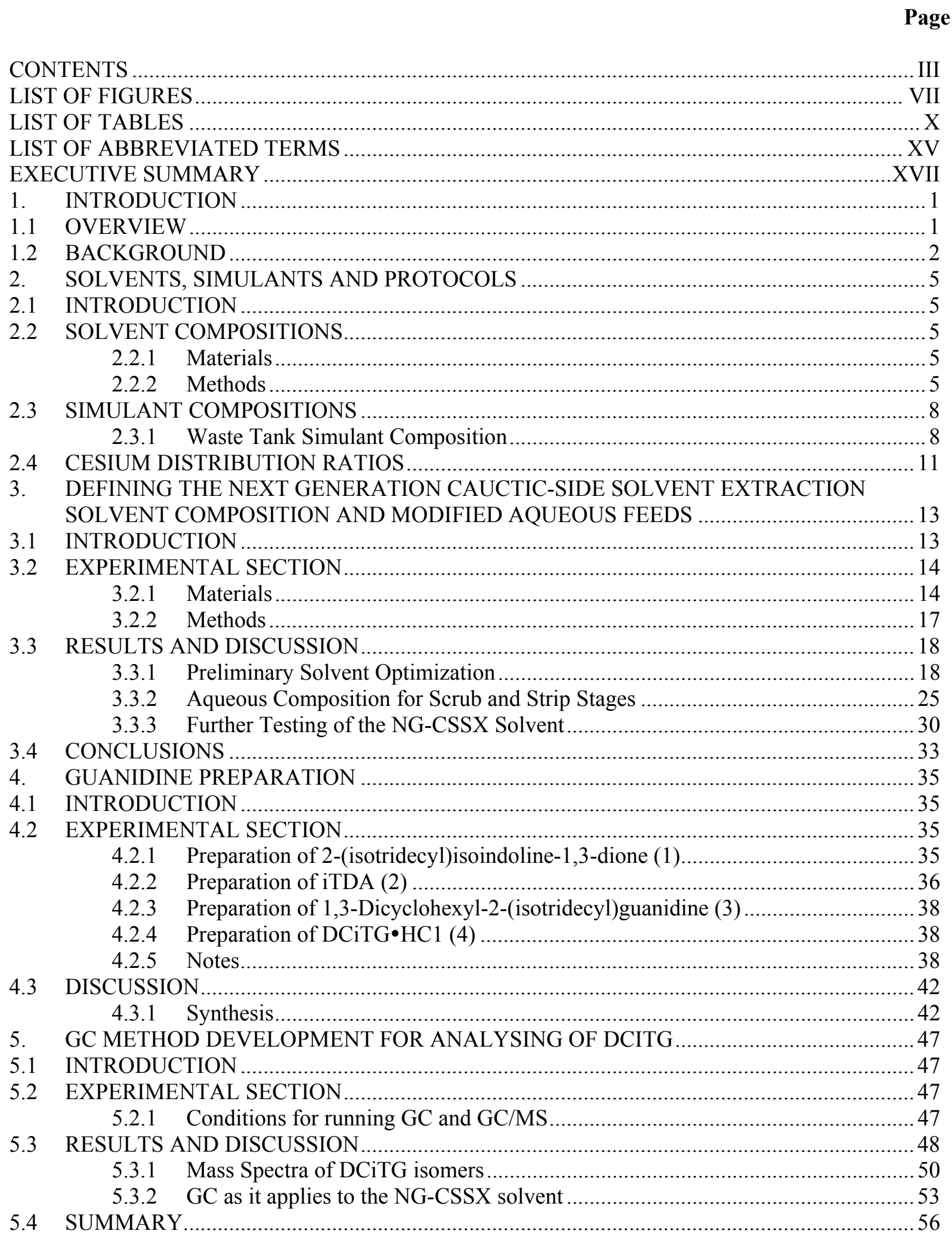

6. MINIMUM PURITY REQUIREMENTS FOR MAXCALIX, DCITG AND DCITG•HCL 


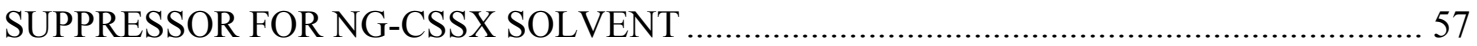

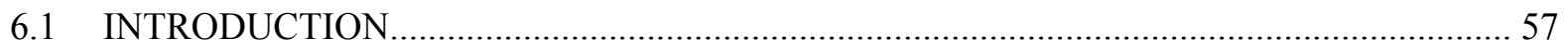

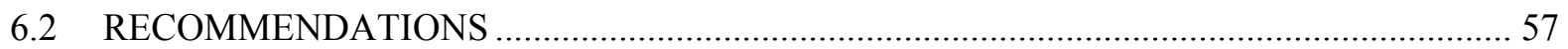

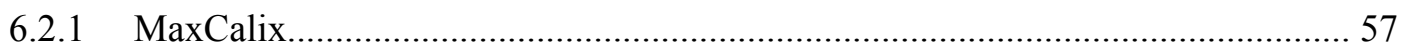

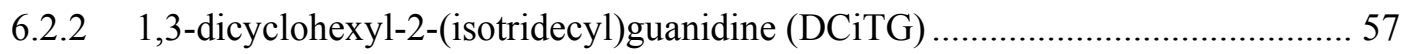

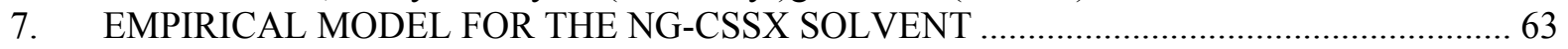

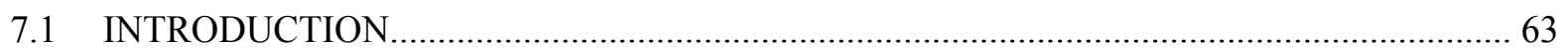

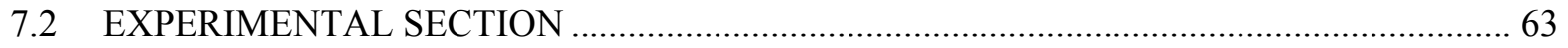

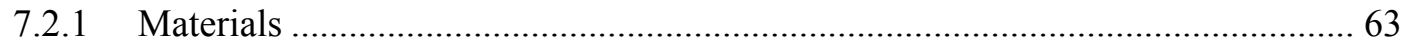

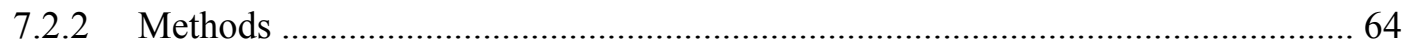

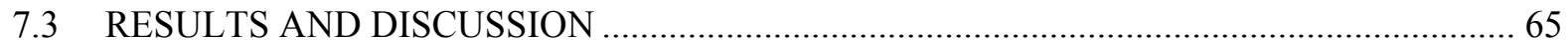

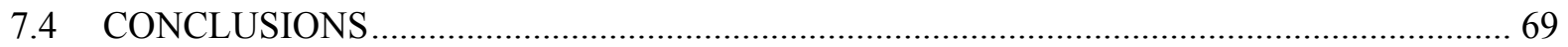

8. EXTRACTION BEHAVIOR OF FINAL SOLVENT UNDER VARYING CONDITIONS ... 71

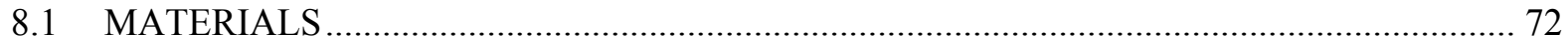

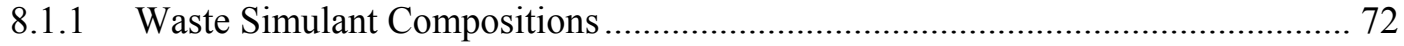

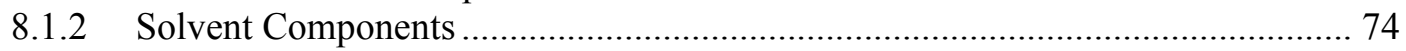

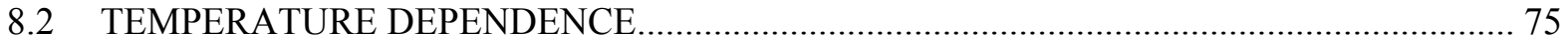

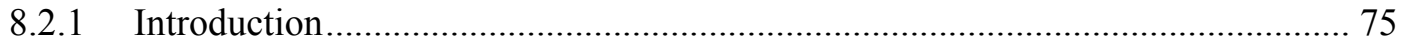

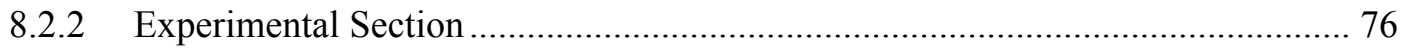

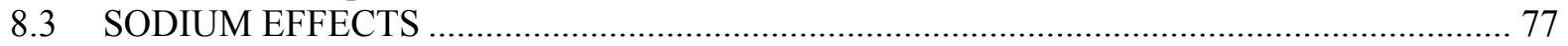

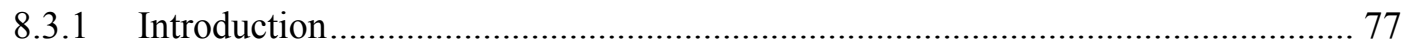

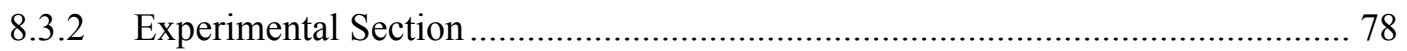

8.4 EFFECT OF POTASSIUM AND CESIUM CONCENTRATION …..................................... 79

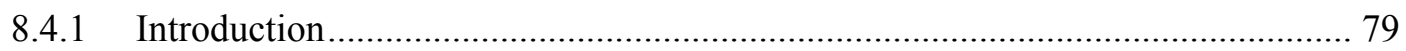

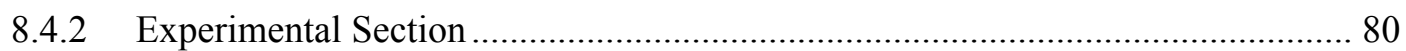

8.5 DETERMINING AQUOUS FEED ALKALINE METAL CONCENTRATION USING INDUCTIVELY COUPLED PLASMA ATOMIC EMISSION SPECTROSCOPY (ICP-AES)

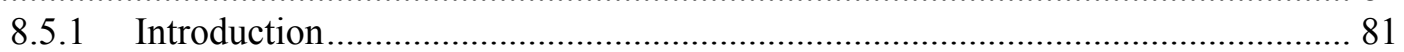

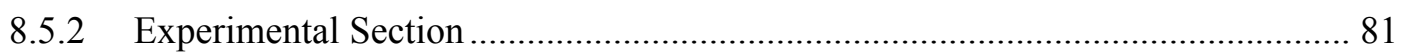

8.6 THIRD PHASE FORMATION AND INTERFACIAL TENSION MEASUREMENTS ......... 83

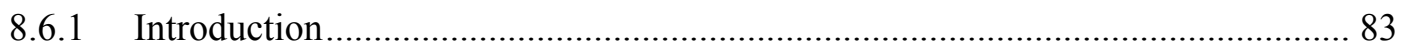

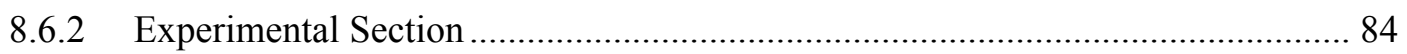

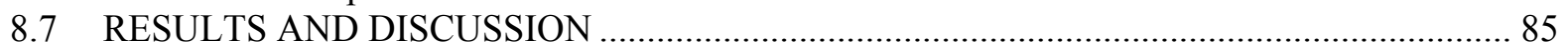

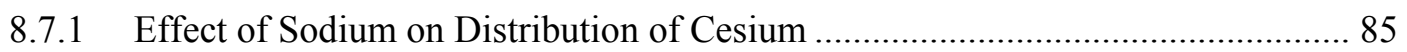

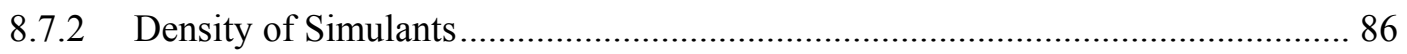

8.7.3 Implications for Waste Decontamination and Processing Rat ............................... 86

8.8 EFFECT OF CESIUM CONCENTRATION ON CESIUM DISTRIBUTION VALUES ........ 87

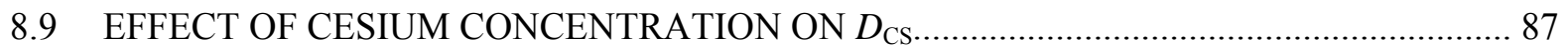

8.10 FOLLOWING THE ALKALINE METALS AND POSSIBLE IMPURITIES IN THE AQUEOUS FLOWSHEET USING INDUCTIVELY COUPLED PLASMA ATOMIC

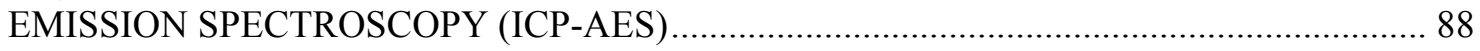

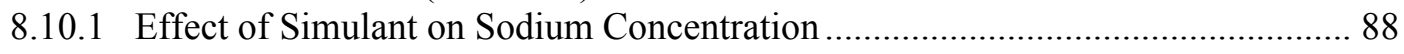

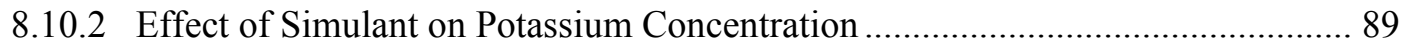

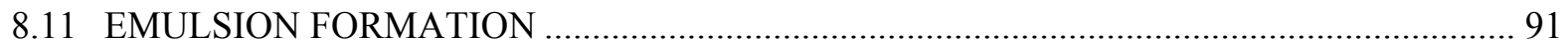

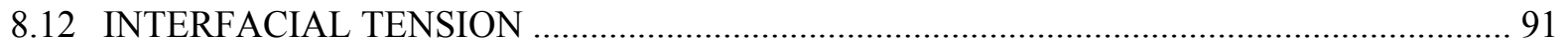

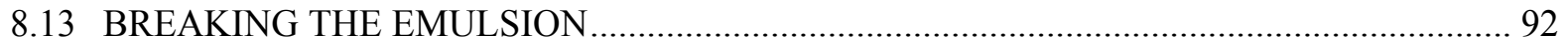

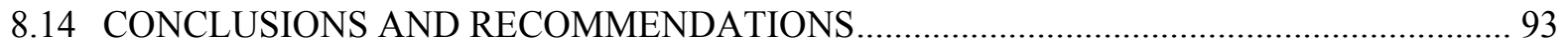

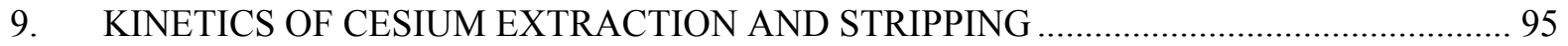

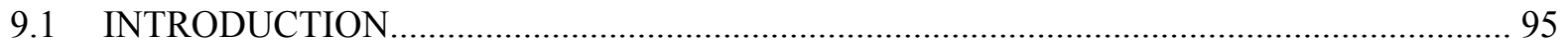

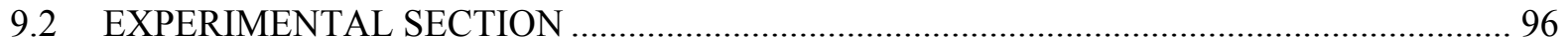




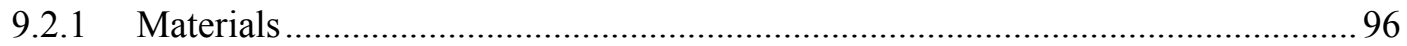

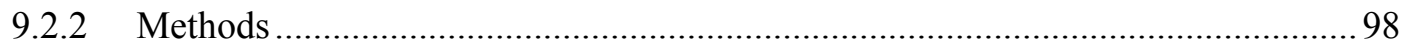

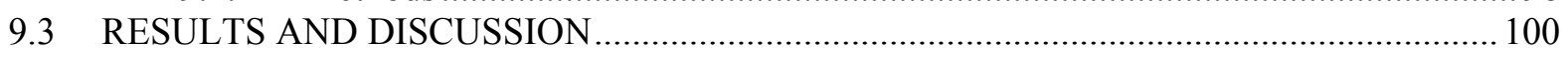

9.3.1 Kinetics of Extraction of MaxCalix and BobCalix C6 ……………………….... 100

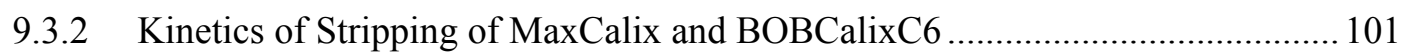

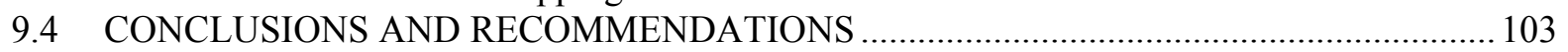

10. NG-CSSX MASS TRANSFER PERFORMANCE IN A PARTIALLY PUMPING 5-CM

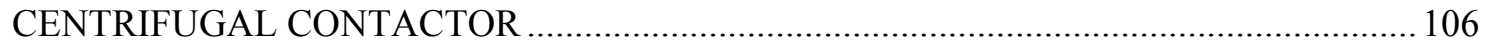

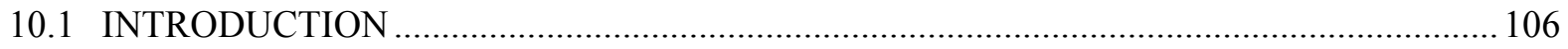

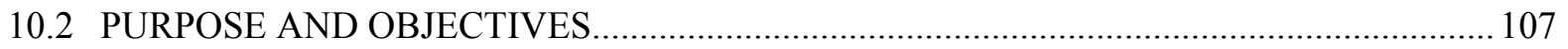

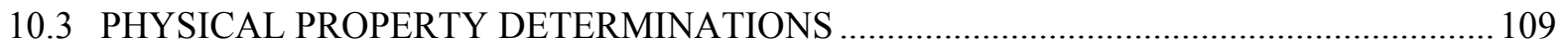

10.4 PRELIMINARY HYDRAULIC PERFORMANCE EVALUATION ……………………….... 114

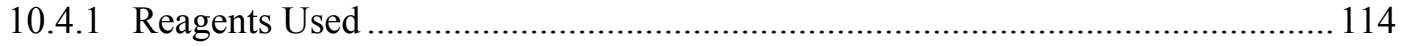

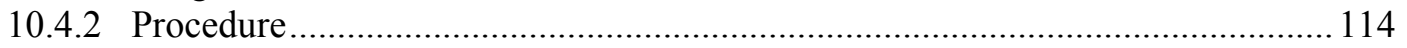

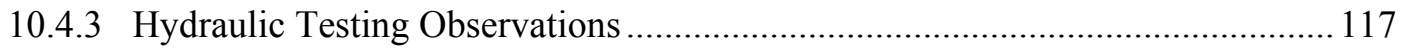

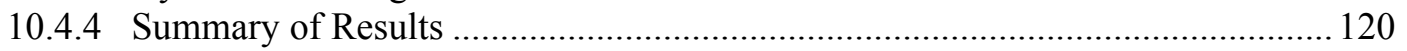

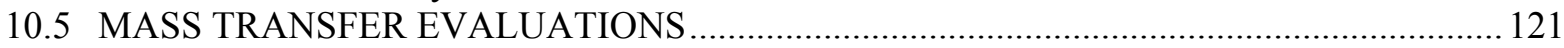

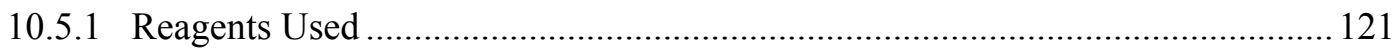

10.5.2 Mass Transfer Test Procedure - Initial Test...................................................... 122

10.5.3 Results of Initial Mass Transfer Test.................................................................. 123

10.5.4 Mass Transfer Test Procedure - Second Test ………………………………..... 124

10.5.5 Results of Second Mass Transfer Test ............................................................ 125

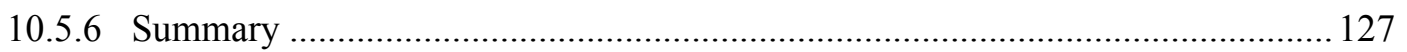

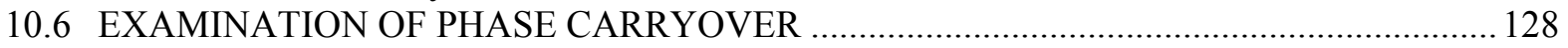

10.6.1 Preliminary testing ……………………………………………………..... 128

10.6.2 Identification of Entrainment Sources............................................................... 130

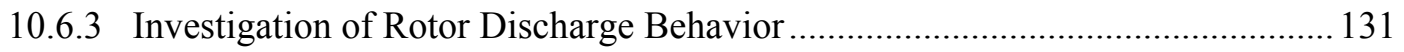

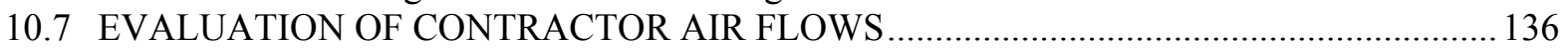

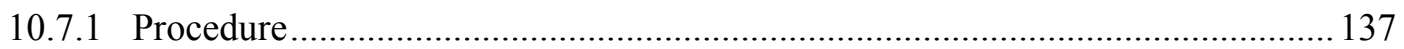

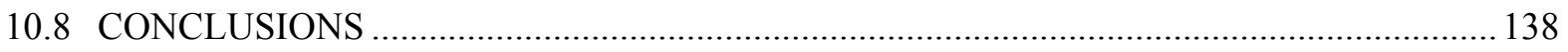

10.8.1 Property Determinations.................................................................................. 138

10.8.2 Contactor Hydraulics................................................................................... 139

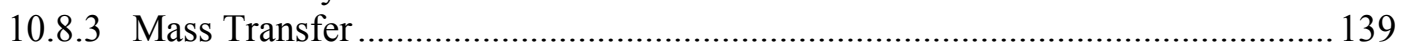

10.8.4 Air Flow and Entrainment Evaluations .......................................................... 139

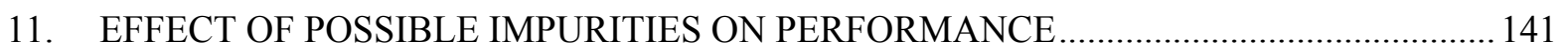

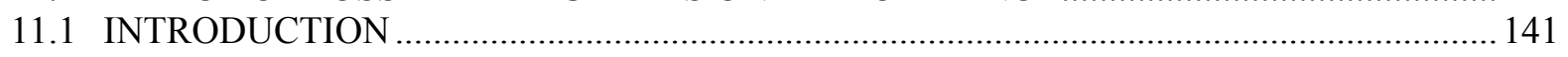

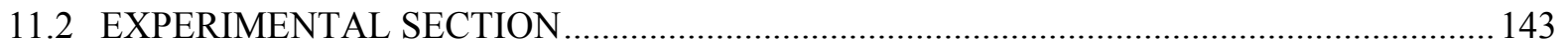

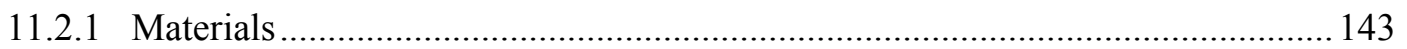

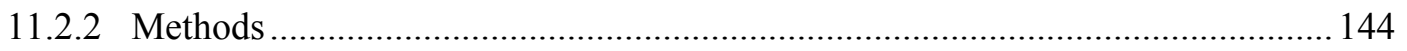

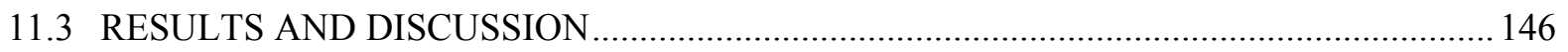

11.3.1 Potential Breakdown Product Impurity Testing ……………………………….... 146

11.3.2 ICP Analysis for SDS and Phosphate Containing Impurities................................ 148

11.4 CONCLUSIONS AND RECOMMENDATIONS ……………………………………….... 151

12. EFFECT OF RADIOLYTIC AND THERMAL TREATMENT ON THE PERFORMANCE OF

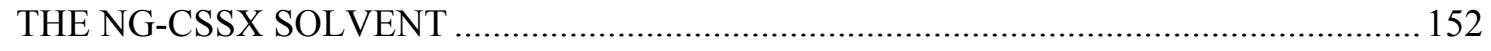

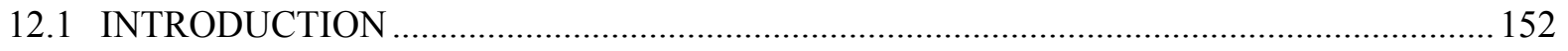

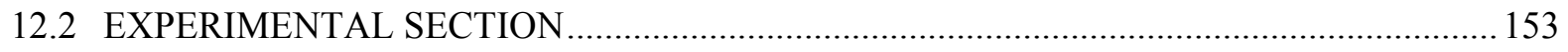

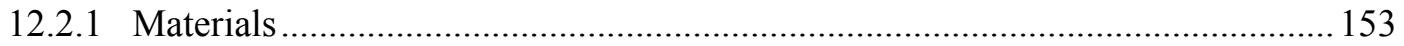

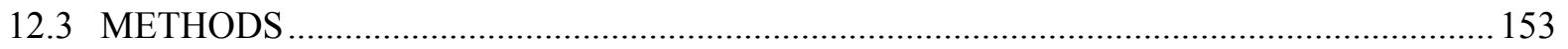

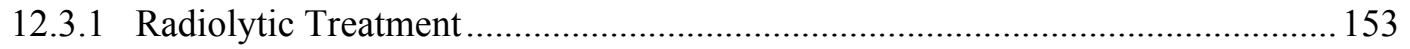

12.3.2 Thermal Treatment ………………………………………………………. 156 
12.3.3 Solvent Loss Due to Leakage......................................................................... 157

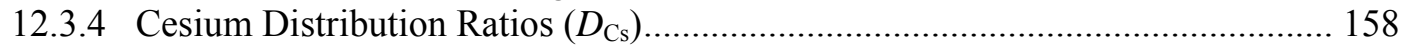

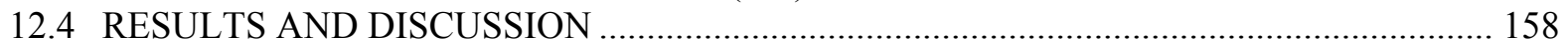

12.4.1 Reference Limits .................................................................................... 158

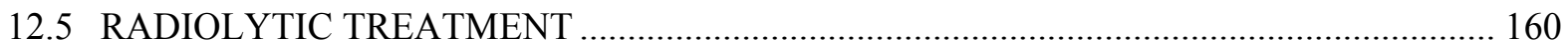

12.5.1 General Observations............................................................................... 160

12.5.2 Coloration of Irradiated Solvent ................................................................... 160

12.5.3 Irradiation During Contact with SRS-15 Simulant ............................................ 161

12.5.4 Irradiation During Contact with $25 \mathrm{mM} \mathrm{NaOH}$................................................ 162

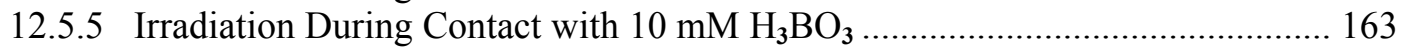

12.5.6 Impact of Radiation on Process Performance ...................................................... 164

12.5.7 Solvent analysis for degratation................................................................... 164

12.5.8 Mass Spec Analysis of NG-CSSX Solvent after Irradiation in Contact with SRS-

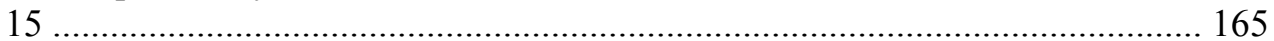

12.5.9 GC Quantification for the degradation of the DCiTGnidine suppressor ............ 166

12.5.10 Degradation product identification ............................................................... 167

12.5.11 Degradation of the Cs-7SB Modifier ............................................................... 167

12.5.12 ESI-MS of partitioned degradation products in strip feed .................................. 167

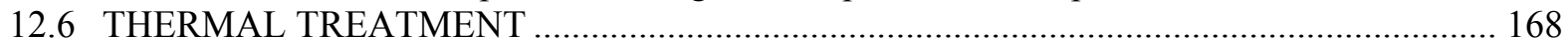

12.6.1 General Observations ............................................................................... 168

12.6.2 Control Solvent ........................................................................................ 168

12.6.3 Thermal Treatment During Contact with SRS-15 Simulant................................ 169

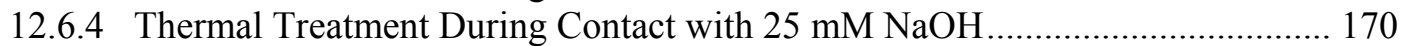

12.6.5 Contact with $10 \mathrm{mM} \mathrm{H}_{3} \mathrm{BO}_{3}$ During Thermal Treatment ................................. 170

12.6.6 Electrospray Mass Spectrometry …………………………………………... 171

12.6.7 Sodium Capacity of Thermally Treated Solvent................................................. 175

12.6.8 Impact of Thermal Treatment on Process Performance....................................... 176

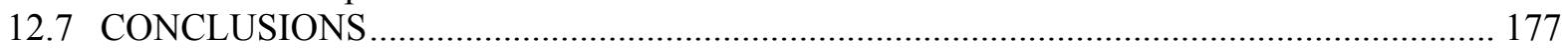

13. SUMMARY OF ACCOMPLISHMENTS ………................................................... 180

13.1 DOCUMENTATION IN REPORTS, PUBLICATIONS, AND PRESENTATIONS ………... 180

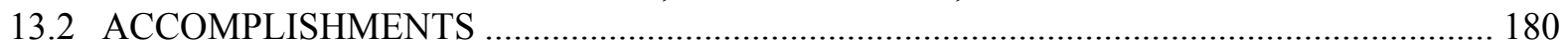

13.2.1 Solvent Chemistry Development ……………………………………….... 180

13.2.2 Flowsheet Behavior of NG-CSSX Solvent ....................................................... 185

13.2.3 Physical Properties of the NG-CSSX Solvent and 5-cm Contactor Performance190

13.3 CONCLUSIONS AND RECOMMENDATIONS........................................................... 192

13.4 PUBLICATIONS, REPORTS, AND PRESENTATIONS RESULTING FROM THIS

RESEARCH AND DEVELOPMENT PROGRAM ……………………………………..... 194

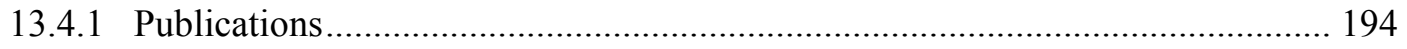

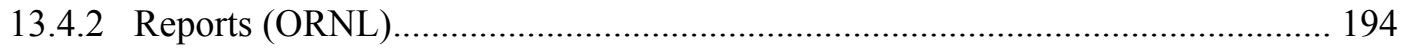

13.4.3 Report (ANL via subcontract) ...................................................................... 196

13.4.4 Presentations ……………………………………………………........ 196

APPENDIX A. QUALITY ASSURANCE IMPLEMENTATION FOR ORNL/TM-2013/224 REV 1

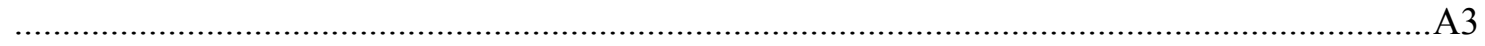

APPENDIX B. ORNL PUBLICATION TRACKING SYSTEM APPROVALS ................................A5

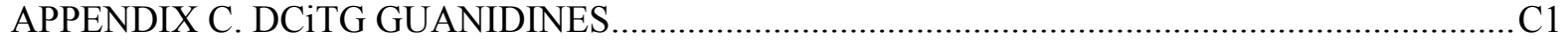

15. REFERENCES. 


\section{LIST OF FIGURES}

Figure

4.1. Synthetic procedure for 1,3-Dicyclohexyl-2-(isotridecyl)guanidine hydrochloride. ...... 37

4.2. GC chromatogram of crude DCiTG with retention time and compound assignments.... 44

4.3. The free base has a decomposition onset at $225^{\circ} \mathrm{C}$ while the hydroxide is at $275^{\circ} \mathrm{C}$ and

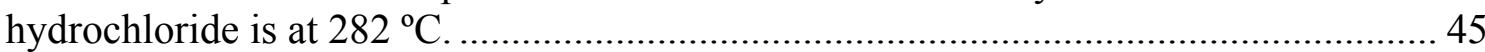

5.1. Calibration of the response factor of DCiTG on the GC/FID Instrument. .................... 49

5.2. Chromatograms from both instruments of synthetic DCiTG mixtures. ......................... 51

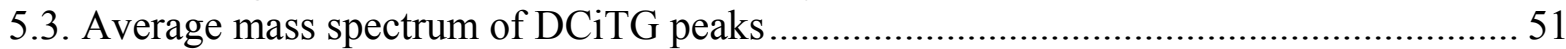

5.4. Mass spectrum for 12 carbon chain DCiTG isomer. ............................................... 52

5.5. Mass spectrum for 13-carbon chain DCiTG isomer................................................ 52

5.6. Mass spectrum for 14-carbon chain DCiTG isomer.............................................. 53

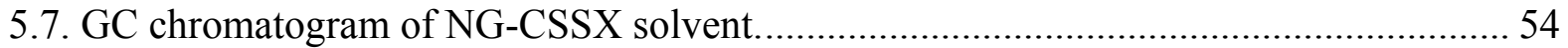

5.8. DCiTG guanidine region in NG-CSSX solvent mixture (DCiTG conc $3 \mathrm{mM}$, area

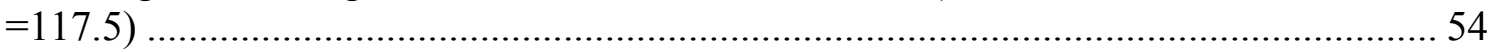

5.9. DCiTG calibration chromatograms (DCiTG conc $2.4 \mathrm{mM}$ and $3.6 \mathrm{mM}$, respectively).. 55

5.10. Comparison of the detector response for DCiTG in NG-CSSX solvent with the

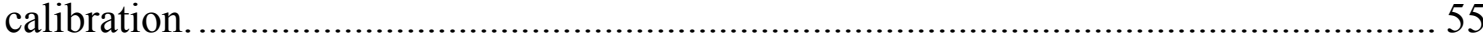

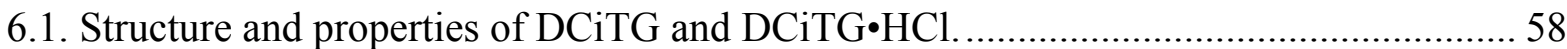

6.2. Graphic of GC oven temperature program conditions for analyzing DCiTG. ............... 60

6.3. Chromatogram of commercial sample of DCiTG (LIX 79 component) from Cognis .... 60

8.1. Emulsion formed upon hand vortexing of the NG-CSSX solvent with boric acid (0.1 M) at an O:A OF $3.75: 1$

8.2. Emulsion boundary formation as a function of guanidine and boric acid concentrations.............91

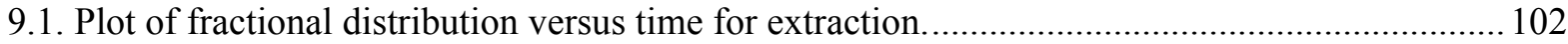

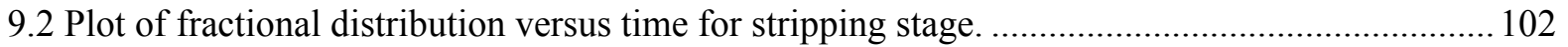

10.1. Transparent housing based on CINC model V-2 contactor.................................... 129

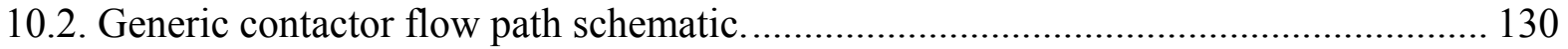

10.3. Photograph of standard CINC V-2 housing (foreground) and modified housing. ...... 135

12.1. NG-CSSX solvent samples after irradiation in contact with SRS-15 simulant (E Series), $25 \mathrm{MM}$ $\mathrm{NAOH}$ (SC Series), and $10 \mathrm{MM} \mathrm{H}_{3} \mathrm{BO}_{3}$ (ST Series).....

12.2. partial mass spectra of the NG-CSSX solvent pre- and postirradiation whilst in contact with SRS-15.

12.3. ESI-MS of NG-CSSX solvent (1,000 fold dilution in MECN) after 3 months thermal treatment whilst in contact with NAOH (25 MM).

12.4. ESI-MS of NG-CSSX solvent (1,000 fold dilution in MECN) after 5 months thermal treatment whilst in contact with NAOH $(25 \mathrm{MM})$.

12.5. ESI-MS of NG-CSSX solvent (1,000 fold dilution in MECN) after 13 months thermal treatment whilst in contact with $\mathrm{NAOH}(25 \mathrm{MM})$.

12.6. ESI-MS of NG-CSSX solvent (1,000 fold dilution in MECN) after 5 months thermal treatment whilst in contact with NAOH (25 MM) followed by consecutive contacts at an O:A of 3.75:1 with NAOH (25 MM), $2 \times \mathrm{H}_{3} \mathrm{BO}_{3}(10 \mathrm{MM})$, and $\mathrm{NAOH}(25 \mathrm{MM})$.

12.7. ESI-MS of NG-CSSX solvent (1,000 fold dilution in MECN) after 13 months thermal treatment whilst in contact with NAOH (25 MM) followed by consecutive contacts at an O:A of 3.75:1

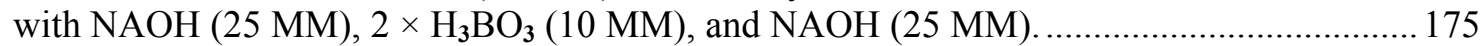

13.1. Using simulant test data provided by ORNL as verified by real-waste test data at SRNL. ...... 186 



\section{LIST OF TABLES}

Table

Page

2.1. BOBCalixC6 CSSX and NG-CSSX solvent components and possible impurities .......... 5

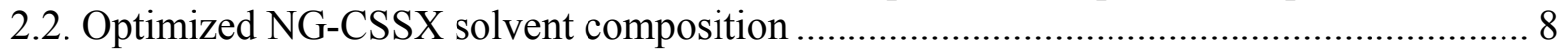

2.3. Target composition of aqueous tank-waste simulants-Part I ................................... 9

2.4. Target compositions of aqueous tank-waste simulants-Part II ................................. 10

3.1 BOBCalixC6 CSSX and NG-CSSX solvent components ....................................... 15

3.2. Target compositions of aqueous tank-waste simulants.......................................... 16

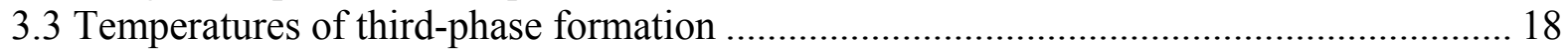

3.4. Cesium distribution ratios comparison between TOA and DCiTG suppressors ............ 19

3.5. Batch ESS contacting results for two BOBCalixC6 CSSX solvents with SRS-15 and Hanford waste simulants ........................................................................................ 20

3.6.1. Batch ESS contacting results one BOBCalixC6 CSSX solvent with differing concentrations of calixerene with SRS-15 ............................................................... 22

3.6.2. Batch ESS contacting results one BOBCalixC6 CSSX solvent with differing concentrations of calixerene with SRS-45 …......................................................... 22

3.7. Batch ESS contacting results for two BOBCalixC6 CSSX solvents with SRS-45 waste

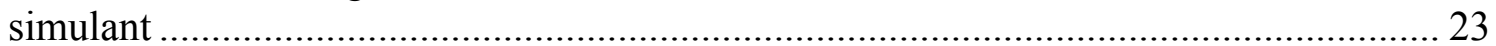

3.8. Batch ESS contacting results for one BOBCalixC6 CSSX solvent (containing TOA) with SRS-15 simulant containing three different concentrations of SDS impurity ............... 24

3.9. Batch ESS contacting results for one BOBCalixC6 CSSX solvent (containing DCiTG) with SRS-15 simulant containing four concentrations of SDS impurity ....................... 24

3.10. Batch ESS contacting for one BOBCalixC6 CSSX solvent (containing DCiTG) with Hanford simulant containing four concentrations of SDS impurity ............................. 25

3.11. Batch ESS contacting results for robustness of the NG-CSSX Solvent. Scrub with $0.1 \mathrm{~N}$ $\mathrm{NaOH}$

3.12. Batch ESS contacting results for one BOBCalixC6 CSSX solvent with different waste

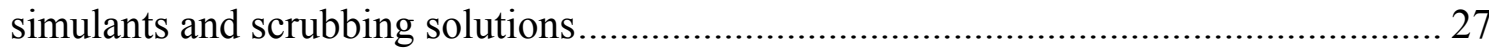

3.13. Batch ESS contacting results one BOBCalixC6 CSSX solvent with differing

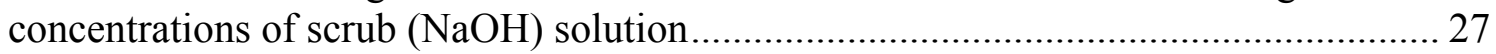

3.14. Relative clarity of the aqueous phase at each stage of the ESS contact ..................... 28

3.15. Batch ESS contacting results for NG-CSSX solvent with SRS-45 and SRS-15 simulants using three stripping solutions....

3.16. Cesium distribution ratios from ESS experiments with SRS-15 waste simulant and stripping boric acid solutions of varying concentrations.

3.17. Cesium distribution ratios from ESS experiments with the SRS-15 and SRS-45 waste simulants using Reduced O:A Ratios

3.18.1. Cycle ESS contacting results for the NG-CSSX solvent with the SRS-15 waste simulant

3.18.2. Cycle ESS contacting results of complete NG-CSSX solvent with the SRS-15 waste

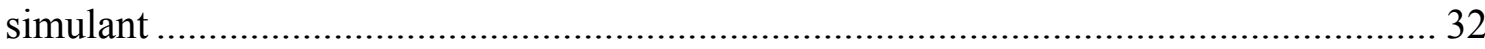

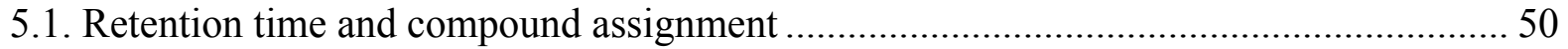

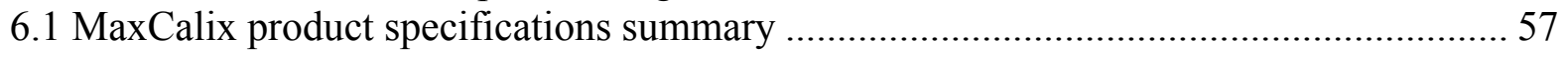

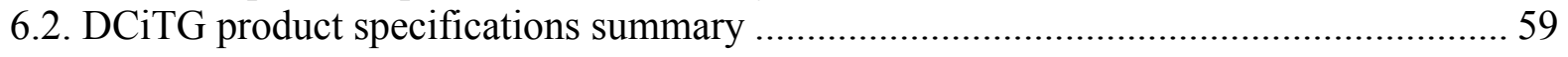


6.3. DCiTG $\bullet \mathrm{HC} 1$ product specifications summary ……........................................................ 59

6.4. Retention times for compounds that may be present in DCiTG...................................... 61

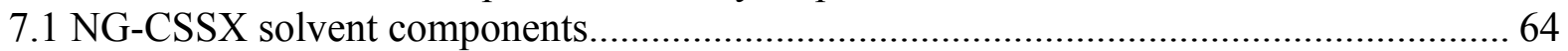

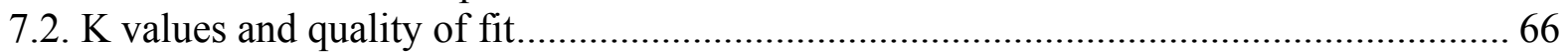

7.3. Sample numbers, compositions, experimental distribution ratios, and predicted

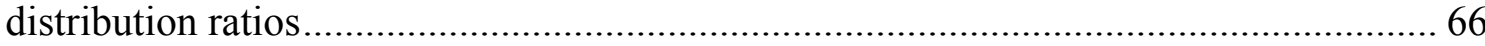

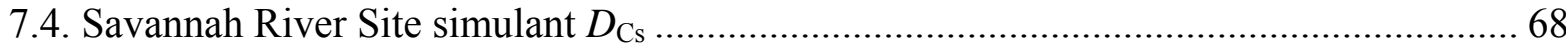

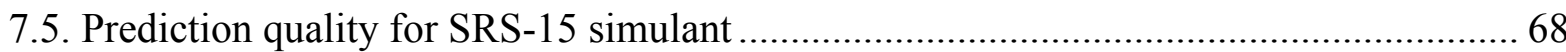

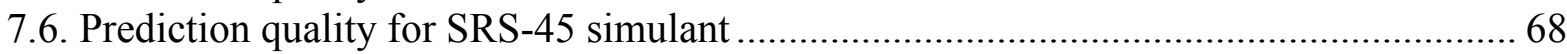

8.1. Target composition of aqueous tank-waste simulants - Part I ...................................... 72

8.2. Target composition of aqueous tank-waste simulants - Part II ..................................... 73

8.3. BOBCalixC6 CSSX and NG-CSSX solvent components ............................................ 75

8.4. Wavelengths used for ICP-AES analysis given along with the correlation of the calibration curve for each analyte and the precision of the measurements $<10 \mathrm{ppm}$...... 83

8.5. ESS performance of the NG-CSSX solvent with varying sodium concentration in SRS

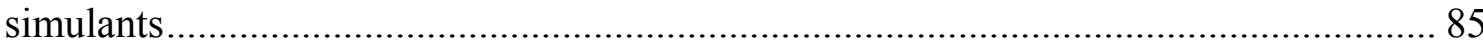

8.6. Densities of SRS simulants used in the experiment.................................................... 86

8.7. ESS performance of the NG-CSSX as cesium concentration in the simulant is increased

.

8.8. ESS performance of the NG-CSSX as simulant changes, the major difference between simulants being the increasing potassium concentration …………............................ 88

8.9. Showing the amount of $\mathrm{Na}, \mathrm{K}$ and $\mathrm{Cs}$ removed from the NG-CSSX solvent (in $\mathrm{mM}$ ) in the scrub and strip stages of the process after being contacted with the various simulants

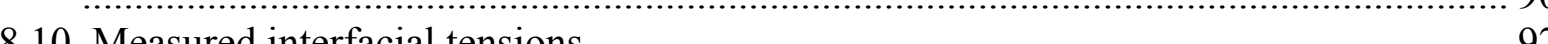

8.10. Measured interfacial tensions .............................................................................. 92

8.11. Attempts at "breaking" the emulsion formed between the NG-CSSX Solvent and boric

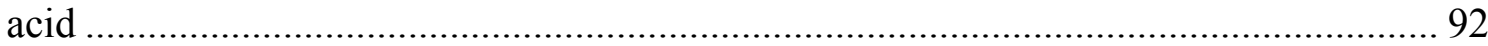

9.1. BOBCalixC6 CSSX and NG-CSSX solvent components ............................................... 96

9.2. Target composition of aqueous tank-waste simulant SRS-15 …................................... 97

9.3. Solvent compositions used in kinetics experiments..................................................... 99

9.4. $D_{\mathrm{Cs}}$ for extraction by BOBCalixC6 CSSX, NG-CSSX, and modified NG-CSSX solvents

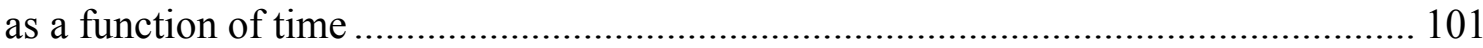

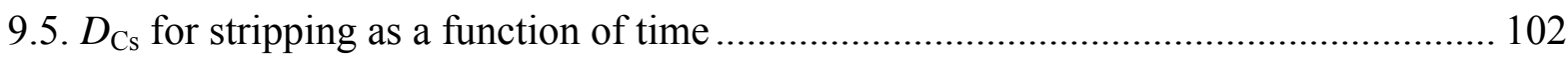

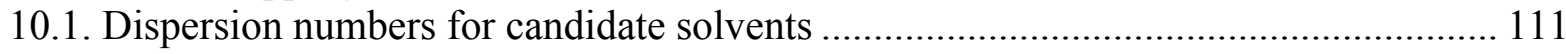

10.2. Effect of modifier concentration on dispersion number ........................................ 112

10.3. Dispersion numbers at modified phase ratios .......................................................... 112

10.4. Effects of scrub solution concentration and phase ratio on dispersion number........... 112

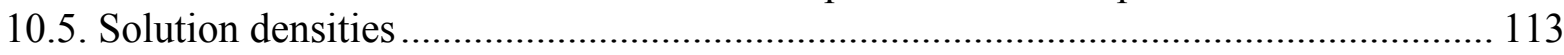

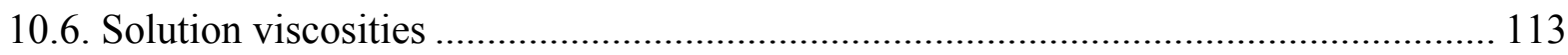

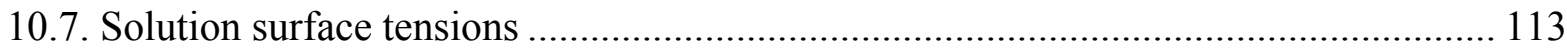

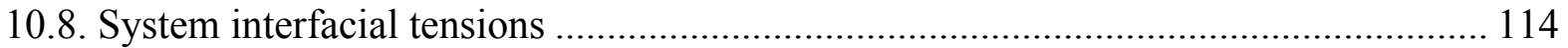

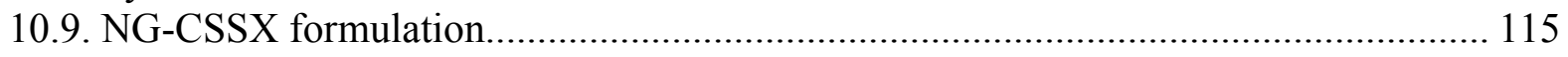

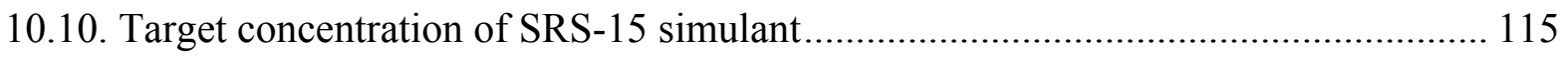

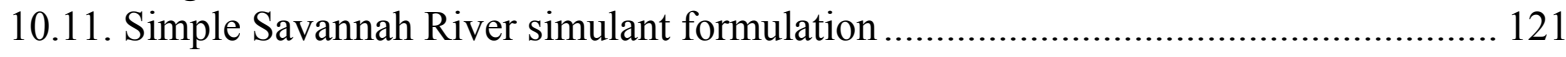

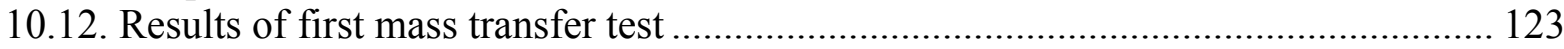

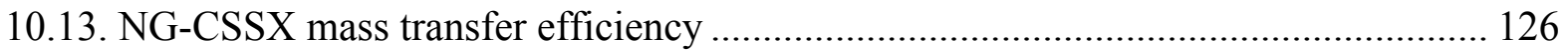




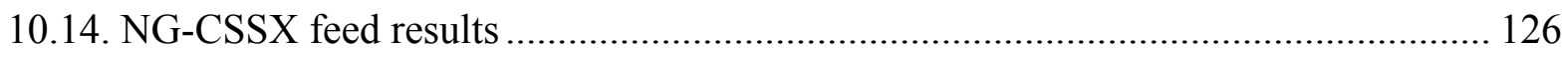

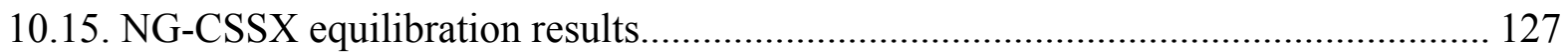

10.16. BOBCalixC6 CSSX mass transfer efficiencies - extraction ................................... 127

10.17. BOBCalixC6 CSSX mass transfer efficiencies - strip ............................................ 127

10.18. Results of solvent carryover evaluations .............................................................. 134

11.1. NG-CSSX solvent components and possible degradation products ........................... 142

11.2. Partial target compositions of aqueous tank-waste simulants used for ICP-AES analysis

11.3. Wavelengths used for ICP-AES analysis given along with the correlation of the calibration curve for each analyte and the precision of the measurements $<10$ ppm.... 145

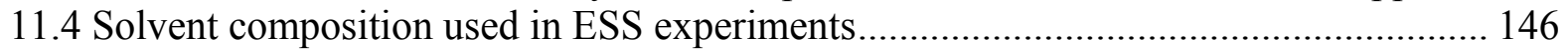

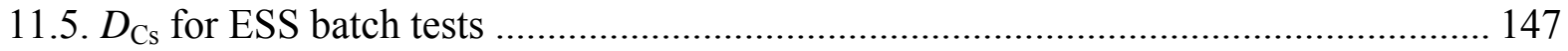

11.6 Showing the amount of sulfur and phosphorus containing impurities removed from the NG-CSSX solvent (in $\mathrm{mM}$ ) in the scrub and strip stages of the process after being

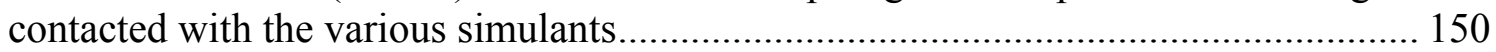

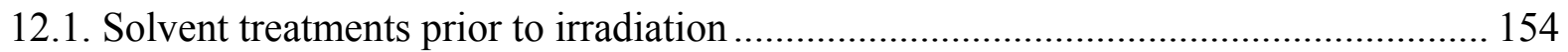

12.2. Doses calculated and determined by Fricke dosimetry.............................................. 155

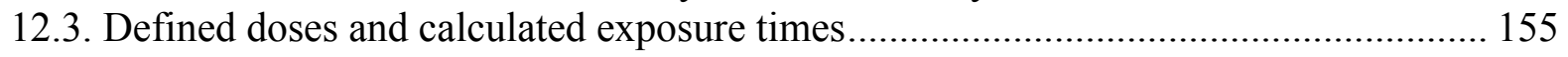

12.4. Solvent Treatments following irradiation ............................................................... 156

12.5. Solvent contacts prior to, and during, thermal treatment.......................................... 156

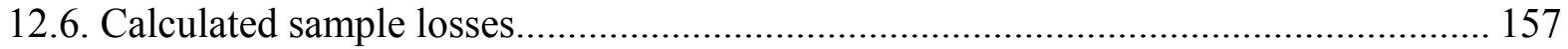

12.7. Solvent contacts following thermal treatment ...................................................... 158

12.8. $D_{\mathrm{Cs}}$ for ESS batch testsa following radiolytic treatment of NG-CSSX solvent in contact

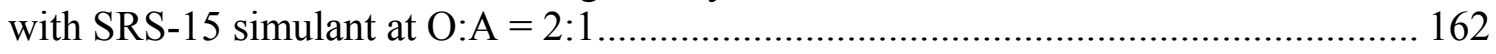

12.9. $D_{\mathrm{CS}}$ for ESS batch testsa following radiolytic treatment of NG-CSSX solvent in contact

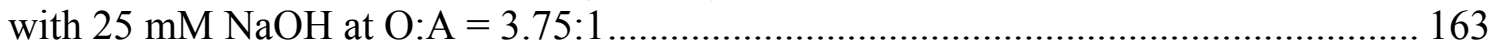

12.10. $D_{\mathrm{Cs}}$ for ESS batch testsa following radiolytic treatment of NG-CSSX solvent in

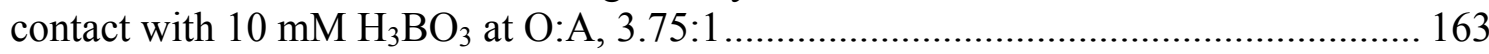

12.11. Sodium distribution ratios for NG-CSSX solvent post radiolytic treatment in contact

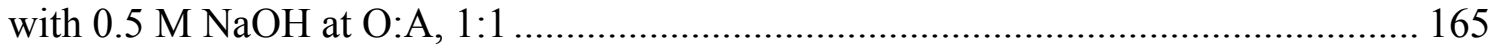

12.12 The DCiTG suppressor concentration before and after solvent irradiation................ 166

12.13 The sec-butylphenol concentration ( $\mathrm{mM}$ ) in the NG-CSSX solvent after irradiation as determined via GC.

12.14: Peaks identified from the ESI-MSof the 1st stripping solution post contact with the NG-CSSX solvent (irradiated in contact with SRS-15 for 50 MRad) ……………...... 168

12.15. $D_{\mathrm{Cs}}$ for ESS batch tests of untreated control solvent for thermal treatment .............. 169

12.16. $D_{\mathrm{Cs}}$ for ESS batch tests of NG-CSSX solvent in continuous contact with SRS-15 simulant at $35^{\circ} \mathrm{C}$

12.17. $D_{\mathrm{Cs}}$ for ESS batch tests of NG-CSSX solvent in continuous contact with $0.025 \mathrm{M}$ $\mathrm{NaOH}$ at $35^{\circ} \mathrm{C}$

12.18. $D_{\mathrm{Cs}}$ for ESS batch tests of NG-CSSX solvent in continuous contact with $0.01 \mathrm{M}$ $\mathrm{H}_{3} \mathrm{BO}_{3}$ at $35 \mathrm{C}$

12.19. The major species observed in the ESI-MS of the NG-CSSX solvent after thermal treatment at $35^{\circ} \mathrm{C}$ whilst in contact with $\mathrm{NaOH}(25 \mathrm{mM})$ for $0,3,5$ and 13 months. . 171

12.20. Sodium loading in the solvents thermally treated with SRS- $15, \mathrm{NaOH}(25 \mathrm{mM})$, and $\mathrm{H}_{3} \mathrm{BO}_{3}(10 \mathrm{mM})$ determined using Na22 by contacting the solvent with $\mathrm{NaOH}(0.5 \mathrm{M})$ 


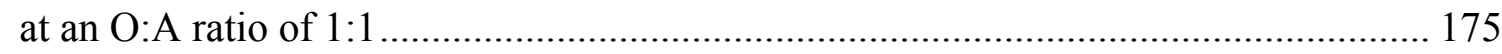

12.21. Assumptions for solvent volumes and temperatures for estimation of thermal degradation

176

12.22. Estimated ESS $D_{\mathrm{Cs}}$ for NG-CSSX solvent inventory over time at $35{ }^{\circ} \mathrm{C} \ldots \ldots \ldots \ldots \ldots . . . . . . .177$

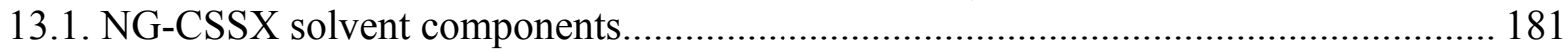





\section{LIST OF ABBREVIATED TERMS}

\begin{tabular}{|c|c|}
\hline ANL & Argonne National Laboratory \\
\hline BEHBCalix & calix[4]arene-bis[4-(2-ethylhexyl)benzocrown-6] \\
\hline BOBCalixC6 & calix[4]arene-bis(tert-octylbenzocrown-6) \\
\hline $\mathrm{CF}$ & concentration factor \\
\hline CINC & Costner Industries Nevada Company \\
\hline Cs-7SB & modifier 1-(2,2,3,3-tetrafluoropropoxy),-3-[4-(sec-butyl)phenoxy]-2-propanol \\
\hline CSSX & Caustic-Side Solvent Extraction \\
\hline DCC & $N, N^{\prime}$-dicyclohexylcarbodiimide \\
\hline DCiTG & 1,3-dicyclohexyl-2-(isotridecyl)guanidine \\
\hline DCiTG•HCl & 1,3-dicyclohexyl-2-(isotridecyl)guanidine hydrochloride \\
\hline$D_{\mathrm{Cs}}$ & cesium distribution ratio, the ratio of organic-to-aqueous phase Cs concentrations \\
\hline DCU & $N, N^{\prime}$-dicyclohexylurea \\
\hline DF & decontamination factor \\
\hline DIAD & diisopropylazodicarboxylate \\
\hline DOE & U.S. Department of Energy \\
\hline DOE-EM & Department of Energy, Office of Environmental Management \\
\hline ESS & extract/scrub/strip \\
\hline $\mathrm{ES}_{2} \mathrm{~S}_{3}$ & extract/scrub $2 x /$ strip $3 x$ \\
\hline ESI-MS & electrospray ionization mass spectrometry \\
\hline FID & flame ionization detector \\
\hline FY & fiscal year \\
\hline GC & gas chromatography \\
\hline ICP-AES & inductively-coupled plasma atomic-emission spectroscopy \\
\hline kGy & kiloGray, absorbed radiation dose equal to $100 \mathrm{krad}$ \\
\hline LIX $^{\circledR} 79$ & commercial (Cognis) guanidine-containing extractant mixture \\
\hline MaxCalix & 1,3-alt-25,27-bis(3,7-dimethyloctyl-1-oxy) calix[4]arene-benzocrown-6 \\
\hline MCU & Modular CSSX Unit \\
\hline NG-CSSX & Next Generation Caustic-Side Solvent Extraction \\
\hline NMR & Nuclear Magnetic Resonance \\
\hline $\mathrm{O}: \mathrm{A}$ & organic-to-aqueous phase volume ratio \\
\hline ORNL & Oak Ridge National Laboratory \\
\hline SBP & 4-sec-butylphenol \\
\hline SDS & sodium dodecyl sulfate \\
\hline SRNL & Savannah River National Laboratory \\
\hline SRR & Savannah River Remediation Co. \\
\hline SRS & Savannah River Site \\
\hline SRS-15 & SRS salt waste with average composition; potassium is $15 \mathrm{mM}$ \\
\hline SRS-45 & SRS salt waste with average composition; potassium is $45 \mathrm{mM}$, bounding conc. \\
\hline SWPF & Salt Waste Processing Facility \\
\hline $\mathrm{TN}$ & Tennessee \\
\hline TOA & Tri- $n$-octylamine \\
\hline VWR & VWR Scientific vendor \\
\hline
\end{tabular}





\section{EXECUTIVE SUMMARY}

This report summarizes the FY 2010 and 2011 accomplishments at Oak Ridge National Laboratory (ORNL) in developing the Next Generation Caustic-Side Solvent Extraction (NG-CSSX) process, referred to commonly as the Next Generation Solvent (NGS), under funding from the U.S. Department of Energy, Office of Environmental Management (DOE-EM), Office of Technology Innovation and Development. The primary product of this effort is a process solvent and preliminary flowsheet capable of meeting a target decontamination factor (DF) of 40,000 for worst-case Savannah River Site (SRS) waste with a concentration factor of 15 or higher in the 18-stage equipment configuration of the SRS Modular Caustic-Side Solvent Extraction Unit (MCU). In addition, the NGCSSX process may be readily adapted for use in the SRS Salt Waste Processing Facility (SWPF) or in supplemental tank-waste treatment at Hanford upon appropriate solvent or flowsheet modifications. Efforts in FY 2010 focused on developing a solvent composition and process flowsheet for MCU implementation. In FY 2011 accomplishments at ORNL involved a wide array of chemical-development activities and testing up through single-stage hydraulic and mass-transfer tests in 5-cm centrifugal contactors. Under subcontract from ORNL, Argonne National Laboratory (ANL) designed a preliminary flowsheet using ORNL cesium distribution data, and Tennessee Technological University confirmed a chemical model for cesium distribution ratios $\left(D_{\mathrm{Cs}}\right)$ as a function of feed composition. Inter laboratory efforts were coordinated with complementary engineering tests carried out (and reported separately) by personnel at Savannah River National Laboratory (SRNL) and Savannah River Remediation (SRR) with helpful advice by Parsons Engineering and General Atomics on aspects of possible SWPF implementation.

Results at ORNL served to characterize the flowsheet chemistry and to reduce corresponding technical uncertainties. Overall, based on the testing results, the chemistry risks for NG-CSSX appear comparable to BOBCalixC6 CSSX prior to its full-scale testing (i.e., implementation in $\mathrm{MCU}$ ), except for one finding, namely the significant and unanticipated partitioning of the NG-CSSX suppressor DCiTG into the aqueous strip solution (10 $\mathrm{mM}$ boric acid). This finding prompted a follow-on research thrust to identify and qualify a new suppressor for the NG-CSSX solvent, which was successful and is reported elsewhere. A more complete summation of the results in this report together with recommendations for further work is given in Chapter 13. The following bullets highlight the major conclusions:

- The preferred solvent composition is $50 \mathrm{mM}$ MaxCalix cesium extractant, $0.50 \mathrm{M}$ Cs-7SB solvent modifier, 3 mM DCiTG solvent suppressor, in Isopar ${ }^{\circledR}$ L diluent; 
- The preferred aqueous process solutions are $25 \mathrm{mM} \mathrm{NaOH}$ scrub solution and $10 \mathrm{mM}$ boric acid strip solution. Improvements to the wash solution were not investigated;

- The preliminary flowsheet is capable of meeting process goals of a DF of 40,000 and CF of 15 in the 18-stage MCU configuration;

- A synthetic preparation and analytical method were developed for the guanidine suppressor;

- The solvent is expected to be thermally and radiolytically stable for 3 years under process conditions;

- Cesium extraction increases with increasing sodium concentration to at least $8 \mathrm{M} \mathrm{Na}$, making it possible to enhance waste processing rate by increasing waste concentration;

- The kinetics of extraction and stripping are significantly faster than for BOBCalixC6 CSSX;

- Impurities have comparable effects as encountered for BOBCalixC6 CSSX;

- Emulsions can form at high guanidine and/or boric acid concentrations, which give low interfacial tensions but which are well outside normal operating concentrations;

- Feed components other than cesium are effectively removed in the scrub section, and the strip effluent primarily consists of cesium in dilute boric acid with traces of sodium and potassium;

- The highest expected cesium concentrations in SRS salt-waste feed will partly compromise the capacity of the $10 \mathrm{mM}$ boric acid strip solution, but performance will be adequate;

- Batch tests show that the solvent maintains adequate performance on cycling 10 times;

- The solvent exhibits a steeper temperature dependence vs the BOBCalixC6 CSSX solvent and will therefore also require temperature control;

- An empirical model predicts cesium distribution ratios within $\pm 16 \%$ for a range of feed compositions;

- The DCiTG suppressor partitions significantly to the aqueous strip solution, leading to follow-on work to replace this suppressor (reported elsewhere);

- Dispersion-number determinations are consistent with adequate contactor hydraulics;

- Single-stage contactor tests with a CINC model V-2 contactor indicate phase separation is good, but not at throughputs approaching the vendor recommended processing capacity;

- Acceptable mass-transfer stage efficiencies in extraction and stripping are noted in singlestage contactor tests with a CINC model V-2 contactor; and

- Contactor improvements can potentially reduce organic carryover into aqueous effluents.

This report fulfills the two programmatic purposes of collecting the initial phase of NG-CSSX development and of documenting the implementation of Quality Assurance (QA) procedures. In the 
2010-2011 timeframe, results were transmitted to the DOE and SRS primarily in the form of "letter reports," as well as two proceedings papers and a journal article. The letter reports provided detail on separate subtopics related to the NG-CSSX development, but they did not represent a complete account of all of the research and were not released to the public. With one exception, dealing with solvent blending (described in a dedicated report), the complete results prior to FY 2012 are collected herein. A list of recommended future development activities that may add further value is also provided, including a straightforward identification of an alternative guanidine suppressor together with further characterization of the identity and fate of degradation products in support of a more informed choice of solvent-wash solution. 



\section{INTRODUCTION}

\subsection{OVERVIEW}

Multilab efforts in fiscal year (FY) 2010 and FY 2011 to develop, scale up, and test the Next Generation Caustic-Side Solvent Extraction (NG-CSSX) process added remarkable potential value to salt-waste processing versus the current baseline BOBCalixC6 CSSX process. Not only is it possible to increase the waste decontamination factor (DF) by 1000 -fold, but years can be subtracted from the waste-processing schedule, and even the burden on downstream operations can be minimized. This report summarizes the FY 2010 through FY 2012 accomplishments at Oak Ridge National Laboratory (ORNL) in developing the NG-CSSX process under funding from the U.S. Department of Energy, Office of Environmental Management (DOE-EM), Office of Technology Innovation and Development. As directed, the primary objective was a process solvent and flowsheet optimized for use in the 18-stage equipment configuration of the Savannah River Site (SRS) Modular Caustic-Side Solvent Extraction Unit (MCU). It was also a long-range objective to enable, with flowsheet modification and possible further solvent optimization, potential use in the SRS Salt Waste Processing Facility (SWPF) or in supplemental waste treatment at Hanford. Efforts in FY 2010 focused on developing a solvent composition and process flowsheet for MCU implementation. In FY 2011, accomplishments at ORNL involved a wide array of chemical-process development and testing, culminating in hydraulic and mass-transfer tests in 5-cm centrifugal contactors. Efforts included selection of the preferred solvent components and concentrations; selection of aqueous feed concentrations; measurement of cesium distribution in extraction/scrub/strip tests as a function of compositional variables, O:A phase ratio, and temperature; synthesis and analytical method development for the guanidine suppressor; determination of thermal and radiolytic solvent stability under process conditions; determination of the effect of sodium concentration in the feed, possibly enabling higher waste-processing rates; development of a strategy for blending used MCU solvent with new NG-CSSX solvent components, thereby avoiding costly disposal of used solvent; examination of the effect of impurities on flowsheet performance; measurement of guanidinie partitioning; physical properties determination of flowsheet solutions; and identification of contactor improvements that will markedly reduce organic carryover into aqueous streams. Under subcontract from ORNL, Argonne National Laboratory (ANL) designed an optimum flowsheet using ORNL cesium distribution data, and Tennessee Technological University confirmed a chemical model for cesium distribution ratios $\left(D_{\mathrm{CS}}\right)$ as a function of feed composition. These efforts were coordinated with complementary engineering tests carried out by personnel at Savannah River National Laboratory (SRNL) and Savannah River Remediation (SRR). Advice was supplied by Parsons Engineering and General Atomics on aspects of possible SWPF 
implementation. A complete list of the accomplishments detailed in this report is given in Chapter 13. A list of recommended future development activities that may add further value is provided. Further characterization of the identity and fate of the degradation products can be used to choose a better solvent-wash solution.

\subsection{BACKGROUND}

Under funding from the DOE-EM Office of Technology Innovation and Development, laboratory efforts since March 2010 have been directed toward developing the NG-CSSX process for cesium removal from alkaline tank wastes ${ }^{1}$. In support of DOE-EM's drive to accelerate processing of tank wastes at the SRS and Hanford site $^{2}$, the laboratory efforts have sought and achieved dramatically increased performance over the original BOBCalixC6 CSSX process, both in decontamination efficiency and waste-processing rate $^{3}$. The resulting much more powerful solvent-extraction process would enable the following potential multisite, multicontractor benefits: life extension of the MCU by raising its DF to $\geq 40,000$; increased processing rate at the SWPF by $50 \%$ or more; and an alternative near-tank modular technology for cesium removal from Hanford tank waste. Research, development, testing, and demonstration activities associated with MCU were conducted in a coordinated program involving SRNL, SRR, and ANL, with Parsons Engineering, and General Atomics providing advice on SWPF.

Program directives focused initially on activities supporting MCU life extension, but subsequently expanded to include potential implementation in the $\mathrm{SWPF}^{1}$. In June 2010, the schedule for implementation at MCU was accelerated by a DOE-EM directive from late 2010 to late 2011, which placed chemical development and engineering testing on a parallel track with an acknowledged risk of discovery and corresponding need for retesting. Over the course of the next year, NG-CSSX development proceeded in support of the accelerated MCU implementation, though in mid FY 2011 plans for MCU implementation were temporarily suspended. In the mid-to Latter part of FY 2011, SRS site planning for FY 2012 began to seriously consider the adoption of NG-CSSX chemistry to boost the throughput of the SWPF sometime after SWPF's planned commissioning. A full-scale countercurrent centrifugal-contactor test of SWPF-NG took place in FY 2012 at the Parsons Technology Center in Aiken, SC. In FY 2013, plans to implement NG in the MCU resumed in an effort to complete the maturation of NG-CSSX featuring a new guanidine suppressor. The maturation plans have driven the completion of the present report and include the documentation of the implemented ORNL QA (see Appendix).

This report is intended to summarize and highlight the accomplishments of ORNL and its subcontractors at ANL, and the confirmations performed by Tennessee Technological University in developing and 
testing the chemistry of NG-CSSX over the period March 2010 to September 2011, as well as evaluate the present status with recommendations for future work. A large body of results was produced in this period, and references to all of the available full reports are given, though some of the results may not have been disseminated to the public. Results of the testing program carried out at the SRNL and SRR are reported separately. 



\section{SOLVENTS, SIMULANTS AND PROTOCOLS}

\subsection{INTRODUCTION}

In this chapter, the compositions of solvents and simulants common to subsequent chapters are presented. The procedure for carrying out batch ESS tests is also described.

\subsection{SOLVENT COMPOSITIONS}

\subsubsection{Materials}

Solvent components were obtained from commercial sources and judged to be of adequate purity for use as received. $N, N^{\prime}$-Dicyclohexyl- $N^{\prime \prime}$-isotridecylguanidine (DCiTG), the active guanidine reagent in Commercial (Cognis) guanidine-containing extractant mixture (LIX ${ }^{\circledR} 79$ ), was supplied by Cognis. Calix[4]arene-bis(tert-octylbenzocrown-6) (BOBCalixC6) was obtained from IBC Advanced Technologies. 1-(2,2,3,3-Tetrafluoropropoxy),-3-[4-(sec-butyl)phenoxy]-2-propanol (Cs-7SB modifier, Lot No. MOD2010-M-2), calix[4]arene-bis[4-(2-ethylhexyl)benzocrown-6] (BEHBCalix), and 1,3-alt25,27-bis(3,7-dimethyloctyl-1-oxy)calix[4]arene-benzocrown-6 (MaxCalix, Lot No. 71-061-15) were obtained from Marshallton Research. Tri-n-octylamine (TOA) was obtained from Aldrich, and Isopar ${ }^{\circledR} \mathrm{L}$ (Lot No. US67377A) was obtained from ExxonMobil. The $N, N^{\prime}$-dicyclohexyl- $N "$-isotridecylguanidine suppressors DCiTG-M2 (Marshallton Research Laboratories, lot no. 79-042-2, using Jarcol ${ }^{\mathrm{TM}} \mathrm{I}-13$ feedstock), used in the irradiation study, and DCiTG-E (synthesized at ORNL, see chapter 4, using EXXAL ${ }^{\circledR} 13$ feedstock), used in the thermal treatment study, were in the form of the hydrochloride salts.

\subsubsection{Methods}

Solvents were prepared by weighing appropriate amounts of extractant, modifier, and suppressor (TOA or LIX 79 guanidine) into volumetric flasks and diluting with Isopar L to the mark. The components are shown in Table 2.1.

Table 2.1. BOBCalixC6 CSSX and NG-CSSX solvent components and possible impurities

\begin{tabular}{lll}
\hline Component & Code & Chemical Name \\
\hline Extractant & MaxCalix & $\begin{array}{l}\text { 1,3-alt-25,27-bis(3,7- } \\
\text { dimethyloctyl-1-oxy) } \\
\text { calix[4]arene- } \\
\text { benzocrown-6 }\end{array}$
\end{tabular}


Table 2.1. BOBCalixC6 CSSX and NG-CSSX solvent components and possible impurities

\begin{tabular}{|c|c|c|c|}
\hline Component & Code & Chemical Name & Structure \\
\hline Extractant & BOBCalixC6 & $\begin{array}{l}\text { Calix[4]arene-bis(tert- } \\
\text { octylbenzocrown-6) }\end{array}$ & \\
\hline Modifier & Cs-7SB & $\begin{array}{l}\text { 1-(2,2,3,3- } \\
\text { Tetrafluoropropoxy)-3- } \\
\text { (4-sec-butylphenoxy)- } \\
\text { 2-propanol }\end{array}$ & \\
\hline Suppressor* & $\begin{array}{l}\text { DCiTG } \\
\text { (LIX 79) }\end{array}$ & $\begin{array}{l}N, N^{\prime} \text {-Dicyclohexyl- } N^{\prime \prime}- \\
\text { isotridecylguanidine }\end{array}$ & \\
\hline Suppressor & TOA & Tri- $n$-octylamine & \\
\hline Diluent & Isopar L & $\begin{array}{l}\mathrm{C}_{12} \text {-isoparaffinic } \\
\text { hydrocarbon }\end{array}$ & \\
\hline $\begin{array}{l}\text { DCiTG } \\
\text { Impurity } 1\end{array}$ & DCU & $N, N^{\prime}$-Dicyclohexylurea & \\
\hline $\begin{array}{l}\text { DCiTG } \\
\text { Impurity } 2\end{array}$ & iTDA & Isotridecylamine & $\begin{array}{c}i \mathrm{C}_{13} \mathrm{H}_{27} \mathrm{NH}_{2} \text { (branched alkyl chain averaging } \\
\text { approximately } 10 \text { carbons long) }\end{array}$ \\
\hline $\begin{array}{l}\text { DCiTG } \\
\text { Impurity } 3\end{array}$ & TCHG & $\begin{array}{l}N, N, N N^{\prime \prime}- \\
\text { Tricyclohexylguanidine }\end{array}$ & \\
\hline $\begin{array}{l}\text { Modifier } \\
\text { Impurity }\end{array}$ & SBP & 4-sec-Butylphenol & \\
\hline
\end{tabular}

*Various suppressors of the same nature were used in different studies, these include the active agent of the LIX 79 (DCiTG L), a commercially available reagent supplied by Cognis; DCiTG-E, a reagent synthesized at ORNL; and DCiTGM1 and DCiTG-M2, two batches of suppressor synthesized using different conditions by Marshallton Research Laboratories.

Solvents were typically prewashed in the following manner: one contact sequentially with $0.010 \mathrm{M} \mathrm{HCl}$, $\mathrm{H}_{2} \mathrm{O}$, then decreasing concentrations of $\mathrm{NaOH}(0.3 \mathrm{M}, 0.1 \mathrm{M}, 0.03 \mathrm{M}$, and $0.01 \mathrm{M})$, and then once or twice with $\mathrm{H}_{2} \mathrm{O}$ until the solution was $\mathrm{pH}$ neutral. In those experiments in which components were used to simulate possible impurities/breakdown products, the simulated decomposition products were not added until after the solvents were washed in order to prevent their washing-out. 


\subsubsection{Optimized solvent}

The optimized NG-CSSX solvent composition, which can achieve the desired DF of 40,000 and concentration factor (CF) of 15 in the MCU equipment configuration - see Chapter 3 of this report assuming at least $90 \%$ stage efficiency $^{4}$, is shown in Table 2.2. The NG-CSSX solvent composition consists of 0.05 M MaxCalix cesium extractant, 0.5 M Cs-7SB modifier, and 0.003 M DCiTG guanidine in Isopar L diluent.

The NG-CSSX solvent has undergone considerable evolution. A mono-crown calixarene called MaxCalix (Tables 2.1, 2.2 and Chapter 3) has been selected as the best choice as the cesium extractant in the NGCSSX solvent. With its high solubility, resistance to third-phase formation, and cesium extraction strength comparable to BOBCalixC6, it has simultaneously allowed higher DFs and higher throughputs to be obtained. Higher DFs can be obtained because of the high extraction $D_{C s}$ values that result from use of much higher calixarene concentrations than was possible with BOBCalixC6 in the baseline BOBCalixC6 CSSX process. An earlier choice of 0.020 M as the concentration for MaxCalix (see Chapter 3) was found in flowsheet calculations not to be high enough to guarantee a cesium DF of $\geq 40,000$ in the MCU configuration $^{4}$. MaxCalix supplants the earlier leading calixarene candidate, called BEHBCalix, which readily formed a third phase at temperatures approaching preferred process temperatures and was therefore deemed not suitable. The Cs-7SB modifier is necessary for solubilizing all other solvent components and extracted species and for increasing cesium extraction strength. It functions well, but increases solvent viscosity and density; hence, its concentration was reduced in NG-CSSX to $0.5 \mathrm{M}$ from the $0.75 \mathrm{M}$ level used in BOBCalixC6 CSSX to improve contactor hydraulics. The guanidine DCiTG enables stripping with boric acid, replacing the TOA used as the suppressor in BOBCalixC6 CSSX . A stronger base is needed as the suppressor in NG-CSSX, because of the higher $\mathrm{pH}$ of the boric acid

stripping conditions. It should be noted that recent experiments ${ }^{5,6}$ have indicated that it would be beneficial to replace DCiTG with a more lipophilic suppressor, as the data shows that DCiTG partitions somewhat to the aqueous boric acid strip solution. 
Table 2.2. Optimized NG-CSSX solvent composition

\begin{tabular}{|c|c|c|c|}
\hline Component & Function & Chemical Name & Structure \\
\hline $\begin{array}{l}\text { MaxCalix } \\
0.05 \mathrm{M}\end{array}$ & Extractant & $\begin{array}{l}\text { 1,3-alternte- } 25,27- \\
\text { bis(3,7-dimethyloctyl-1- } \\
\text { oxy) calix[4]arene- } \\
\text { benzocrown-6 }\end{array}$ & \\
\hline $\begin{array}{l}\text { Cs-7SB } \\
0.5 \mathrm{M}\end{array}$ & Modifier & $\begin{array}{l}\text { 1-(2,2,3,3- } \\
\text { Tetrafluoropropoxy)-3- } \\
\text { (4-sec-butylphenoxy)-2- } \\
\text { propanol }\end{array}$ & \\
\hline $\begin{array}{l}\text { DCiTG } \\
0.003 \mathrm{M}\end{array}$ & Suppressor & $\begin{array}{l}N, N^{\prime} \text {-Dicyclohexyl- } N " \text { - } \\
\text { isotridecylguanidine }\end{array}$ & \\
\hline Isopar L & Diluent & $\begin{array}{l}\mathrm{C}_{12} \text {-isoparaffinic } \\
\text { hydrocarbon }\end{array}$ & \\
\hline
\end{tabular}

\subsection{SIMULANT COMPOSITIONS}

Targeted tank-waste simulant compositions are given in Tables 2.3 and 2.4 according to preparative methods described in detail in earlier reports ${ }^{7,8}$. Constituent species are named in the table as the chemical forms added, not necessarily to be taken as the forms actually present after mixing. Appropriate amounts of reagent-grade chemicals were weighed into volumetric flasks in the order prescribed. Significant precipitate formed upon adding the $\mathrm{NaOH}$, which is considered normal, and the mixture was stirred for several hours and then allowed to settle, normally for a few days before use. Actual component concentrations therefore may differ from the calculated concentrations ${ }^{7}$. The SRS-15 simulant is designed to represent the average SRS tank-waste composition ${ }^{9}$. The SRS-45 simulant has the same composition, except that $0.030 \mathrm{M}$ more $\mathrm{KNO}_{3}$ has been added to represent the upper bound of $0.045 \mathrm{M}$ for the potassium concentration. The Hanford simulant corresponds to Hanford tank 241-AP-108 normalized to $6 \mathrm{M}$ sodium ( simulant $\# 6^{8,9}$ ). It represents a severe Hanford case in terms of high potassium concentration and correspondingly lowest $D_{C s}$, giving the most difficulty in achieving a high DF.

\subsubsection{Waste Tank Simulant Composition}

Constituent species are named in the table as the chemical forms added, not necessarily to be taken as the forms actually present after mixing. Appropriate amounts of reagent-grade chemicals were weighed into volumetric flasks. Significant precipitate formed upon adding the $\mathrm{NaOH}$, which is considered normal, and the mixture was stirred for several hours and then allowed to settle at least for a few days before use. 
Actual component concentrations therefore may differ from the concentrations tabulated ${ }^{7}$. The SRS-15 simulant is designed to represent the average SRS tank-waste composition? 9

\subsubsection{Aqueous Solution Preparation}

Scrub solution $(0.025 \mathrm{M} \mathrm{NaOH})$ and other $\mathrm{NaOH}$ solutions were prepared by dilution of $1.0 \mathrm{M} \mathrm{NaOH}$ standard solution (VWR). Strip solution $\left(0.010 \mathrm{M} \mathrm{H}_{3} \mathrm{BO}_{3}\right)$ was obtained by dilution of a $0.1 \mathrm{M} \mathrm{H}_{3} \mathrm{BO}_{3}$ stock solution prepared from lab-grade $\mathrm{H}_{3} \mathrm{BO}_{3}$ (min 95.5\%, Sigma Aldrich). Solutions of $\mathrm{HCl}$ were made from 1.0 M HCl standard solution (Baker). Water for preparation of all aqueous solutions was first distilled and then deionized using a Milli- $\mathrm{Q}^{\circledR}$ gradient A10 filtering system equipped with a Quantum ${ }^{\mathrm{TM}}$ Ex Ultrapure Organex Cartridge (18.2 $\mathrm{M} \Omega \bullet \mathrm{cm}$ at $25^{\circ} \mathrm{C}$, total organic content $\left.4 \mathrm{ppb}\right)$.

The major components of targeted tank-waste simulant compositions are given in Tables 2.3 and 2.4.

Table 2.3. Target composition of aqueous tank-waste simulants-Part I

\begin{tabular}{|c|c|c|c|c|}
\hline Analyte or Species & $\begin{array}{l}\text { SRS-9 } \\
\mathrm{mol} / \mathrm{L}\end{array}$ & $\begin{array}{c}\mathrm{SRS}-12 \\
\mathrm{~mol} / \mathrm{L}\end{array}$ & $\begin{array}{c}\text { SRS-15 } \\
\mathrm{mol} / \mathrm{L}\end{array}$ & $\begin{array}{c}\text { SRS-19 } \\
\mathrm{mol} / \mathrm{L}\end{array}$ \\
\hline \multicolumn{5}{|l|}{ Principal constituents: } \\
\hline $\mathrm{Al}$ as $\mathrm{Al}(\mathrm{OH})_{4}^{-}$ & 0.175 & 0.255 & 0.280 & 0.340 \\
\hline $\mathrm{Cl}^{-}$ & $1.5 \times 10^{-2}$ & $1.9 \times 10^{-2}$ & $2.4 \times 10^{-2}$ & $2.9 \times 10^{-2}$ \\
\hline $\mathrm{CO}_{3}{ }^{2-}$ (TIC) & 0.075 & 0.120 & 0.150 & 0.182 \\
\hline $\mathrm{Cs}^{+}$(Total) & $8.8 \times 10^{-5}$ & $1.1 \times 10^{-4}$ & $1.4 \times 10^{-4}$ & $1.7 \times 10^{-4}$ \\
\hline $\mathrm{K}^{+}$ & 0.009 & 0.012 & 0.015 & 0.019 \\
\hline $\mathrm{Na}^{+}$ & 3.50 & 4.50 & 5.60 & 6.80 \\
\hline $\mathrm{NO}_{2}^{-}$ & 0.312 & 0.402 & 0.500 & 0.608 \\
\hline $\mathrm{NO}_{3}^{-}$ & 1.27 & 1.63 & 2.03 & 2.50 \\
\hline $\mathrm{OH}^{-}$(Free) & 1.29 & 1.66 & 2.06 & 2.50 \\
\hline $\mathrm{SO}_{4}{ }^{2-}$ & 0.088 & 0.112 & 0.140 & 0.170 \\
\hline \multicolumn{5}{|l|}{ Minor inorganic constituents: } \\
\hline $\operatorname{Ag}(\mathrm{I})$ & $5.8 \times 10^{-8}$ & $7.5 \times 10^{-8}$ & $9.3 \times 10^{-8}$ & $1.1 \times 10^{-7}$ \\
\hline $\mathrm{CrO}_{4}^{2-}$ & $8.8 \times 10^{-4}$ & $1.1 \times 10^{-3}$ & $1.4 \times 10^{-3}$ & $1.7 \times 10^{-3}$ \\
\hline $\mathrm{Cu}(\mathrm{II})$ & $1.4 \times 10^{-5}$ & $1.8 \times 10^{-5}$ & $2.3 \times 10^{-5}$ & $2.8 \times 10^{-5}$ \\
\hline $\mathrm{F}^{-}$ & $1.8 \times 10^{-2}$ & $2.2 \times 10^{-2}$ & $2.8 \times 10^{-2}$ & $3.4 \times 10^{-2}$ \\
\hline $\mathrm{Fe}(\mathrm{III})$ & $1.6 \times 10^{-5}$ & $2.1 \times 10^{-5}$ & $2.6 \times 10^{-5}$ & $3.2 \times 10^{-5}$ \\
\hline $\mathrm{Hg}(\mathrm{II})$ & $1.6 \times 10^{-7}$ & $2.0 \times 10^{-7}$ & $2.5 \times 10^{-7}$ & $3.0 \times 10^{-7}$ \\
\hline $\mathrm{MoO}_{4}{ }^{2-}$ & $4.4 \times 10^{-5}$ & $5.6 \times 10^{-5}$ & $7.0 \times 10^{-5}$ & $8.5 \times 10^{-5}$ \\
\hline $\mathrm{NH}_{3}$ & $6.2 \times 10^{-4}$ & $8.0 \times 10^{-4}$ & $1.0 \times 10^{-3}$ & $1.2 \times 10^{-3}$ \\
\hline $\mathrm{Pb}(\mathrm{II})$ & $6.2 \times 10^{-6}$ & $8.0 \times 10^{-6}$ & $1.0 \times 10^{-5}$ & $1.2 \times 10^{-5}$ \\
\hline $\operatorname{Pd}(\mathrm{II})$ & $2.4 \times 10^{-6}$ & $3.0 \times 10^{-6}$ & $3.8 \times 10^{-6}$ & $4.6 \times 10^{-6}$ \\
\hline $\mathrm{PO}_{4}^{3-}$ & $4.4 \times 10^{-3}$ & $5.6 \times 10^{-3}$ & $7.0 \times 10^{-3}$ & $8.5 \times 10^{-3}$ \\
\hline $\mathrm{Rh}(\mathrm{III})$ & $1.2 \times 10^{-6}$ & $1.6 \times 10^{-6}$ & $2.0 \times 10^{-6}$ & $2.4 \times 10^{-6}$ \\
\hline $\mathrm{Ru}(\mathrm{III})$ & $5.1 \times 10^{-6}$ & $6.5 \times 10^{-6}$ & $8.1 \times 10^{-6}$ & $9.8 \times 10^{-6}$ \\
\hline $\mathrm{Si}(\mathrm{IV})$ & $1.9 \times 10^{-2}$ & $2.4 \times 10^{-2}$ & $3.0 \times 10^{-2}$ & $3.6 \times 10^{-2}$ \\
\hline $\mathrm{Sn}(\mathrm{II})$ & $1.2 \times 10^{-5}$ & $1.6 \times 10^{-5}$ & $2.0 \times 10^{-5}$ & $2.4 \times 10^{-5}$ \\
\hline
\end{tabular}


Table 2.3. Target composition of aqueous tank-waste simulants-Part I

\begin{tabular}{lcccc}
\hline \multicolumn{1}{c}{ Analyte or Species } & $\begin{array}{c}\text { SRS-9 } \\
\mathbf{m o l} / \mathbf{L}\end{array}$ & $\begin{array}{c}\text { SRS-12 } \\
\mathbf{m o l} / \mathbf{L}\end{array}$ & $\begin{array}{c}\text { SRS-15 } \\
\mathbf{m o l} / \mathbf{L}\end{array}$ & $\begin{array}{c}\text { SRS-19 } \\
\mathbf{m o l} / \mathbf{L}\end{array}$ \\
\hline $\begin{array}{l}\text { Minor organic constituents: } \\
n \text {-Butanol }\end{array}$ & $1.7 \times 10^{-5}$ & $2.2 \times 10^{-5}$ & $2.7 \times 10^{-5}$ & $3.3 \times 10^{-5}$ \\
Tri- $n$-butylphosphate (TBP) & $1.2 \times 10^{-6}$ & $1.5 \times 10^{-6}$ & $1.9 \times 10^{-6}$ & $2.3 \times 10^{-6}$ \\
$\begin{array}{l}\text { Di- } n \text {-butylphosphate (DBP) } \\
\text { Mono- } n \text {-butylphosphate }\end{array}$ & $7.5 \times 10^{-5}$ & $9.6 \times 10^{-5}$ & $1.2 \times 10^{-4}$ & $1.4 \times 10^{-4}$ \\
(MBP) & $1.0 \times 10^{-4}$ & $1.3 \times 10^{-4}$ & $1.6 \times 10^{-4}$ & $1.9 \times 10^{-4}$ \\
Formate $\left(\mathrm{HCO}_{2}{ }^{-}\right)$ & $2.1 \times 10^{-2}$ & $2.6 \times 10^{-2}$ & $3.3 \times 10^{-2}$ & $4.0 \times 10^{-2}$ \\
Oxalate $\left(\mathrm{C}_{2} \mathrm{O}_{4}{ }^{2-}\right)$ & $5.0 \times 10^{-3}$ & $6.4 \times 10^{-3}$ & $8.0 \times 10^{-3}$ & $9.7 \times 10^{-3}$ \\
Trimethylamine & $1.1 \times 10^{-4}$ & $1.4 \times 10^{-4}$ & $1.7 \times 10^{-4}$ & $2.1 \times 10^{-4}$ \\
\hline
\end{tabular}

Table 2.4. Target compositions of aqueous tank-waste simulants-Part II

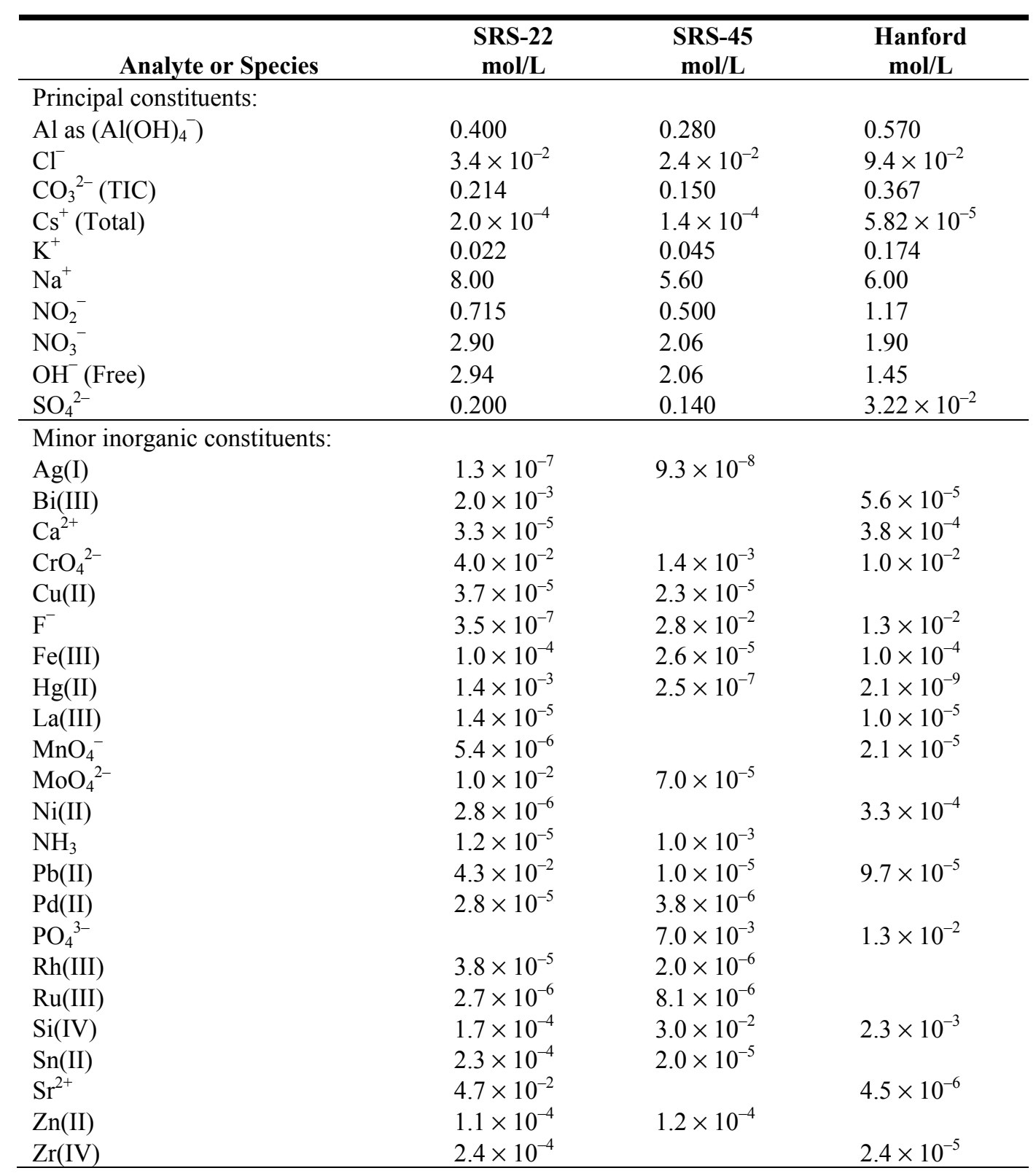


Table 2.4. Target compositions of aqueous tank-waste simulants-Part II

\begin{tabular}{lccc}
\hline \multicolumn{1}{c}{ Analyte or Species } & $\begin{array}{c}\text { SRS-22 } \\
\mathbf{m o l} / \mathbf{L}\end{array}$ & $\begin{array}{c}\text { SRS-45 } \\
\mathbf{~ m o l} / \mathbf{L}\end{array}$ & $\begin{array}{c}\text { Hanford } \\
\text { mol/L }\end{array}$ \\
\hline Minor organic constituents: & & & \\
$n$-Butanol & $3.8 \times 10^{-5}$ & $2.7 \times 10^{-5}$ & \\
Tri- $n$-butylphosphate (TBP) & $2.7 \times 10^{-6}$ & $1.9 \times 10^{-6}$ & \\
Di- $n$-butylphosphate (DBP) & $1.7 \times 10^{-4}$ & $1.2 \times 10^{-4}$ & \\
Mono- $n$-butylphosphate (MBP) & $2.3 \times 10^{-4}$ & $1.6 \times 10^{-4}$ & \\
Formate $\left(\mathrm{HCO}_{2}{ }^{-}\right)$ & $4.7 \times 10^{-2}$ & $3.3 \times 10^{-2}$ & \\
Oxalate $\left(\mathrm{C}_{2} \mathrm{O}_{4}{ }^{2-}\right)$ & $1.1 \times 10^{-4}$ & $8.0 \times 10^{-3}$ & $1.0 \times 10^{-2}$ \\
Trimethylamine & $2.4 \times 10^{-4}$ & $1.7 \times 10^{-4}$ & \\
\hline
\end{tabular}

Further details on preparation and minor components can be found elsewhere ${ }^{9,10}$. The SRS-15 simulant represents the average SRS tank-waste composition; it has a concentration of potassium of $15 \mathrm{mM}$. The SRS-45 simulant has the same composition with added $\mathrm{KNO}_{3}$ to reach a total potassium concentration of $45 \mathrm{mM}$. The Hanford simulant corresponds to Hanford tank 241-AP-108 normalized to $6 \mathrm{M}$ sodium.

The aqueous phase used in the ESS tests was the SRS tank-waste simulant SRS-15, whose composition and preparation has been described in detail previously $y^{3,7,9}$.

\subsection{CESIUM DISTRIBUTION RATIOS}

Cesium distribution ratios with simulants were obtained in duplicate in a manner similar to that described previously $^{7,8,10,11}$, with one extraction followed by two scrubs of $0.025 \mathrm{M} \mathrm{NaOH}$ and three strips of 0.010 $\mathrm{M} \mathrm{H}_{3} \mathrm{BO}_{3}$ (i.e., $\mathrm{ES}_{2} \mathrm{~S}_{3}$ ). Phases were contacted in polypropylene micro-tubes mounted by clips on a disk rotated for 60 minutes for extractions and 45 minutes for scrubs and strips. The solutions were contacted inside of an air-box maintained at a temperature of $25 \pm 0.2{ }^{\circ} \mathrm{C}$. After the contacting period, the tubes were centrifuged for 5 minutes at 3000 RPM at the experimental temperature. An appropriate aliquot of each phase was subsampled and counted using a Packard Cobra II Auto-Gamma counter. A spike of Cs137 was added to the second and third aqueous strip solutions, owing to the low number of counts remaining after the each strip. To keep samples at the equilibration temperature for sampling, the samples were left in a temperature-controlled centrifuge and removed individually for subsampling. Based on the agreement between duplicate samples, the precision of $D_{C s}$ values is $\pm 5 \%$ (extraction and scrub), $\pm 10 \%$ (first strip), and $\pm 30 \%$ (second and third strips). Values of $D_{C s}$ are given as the ratio of the backgroundcorrected volumetric count rates of the radioisotope in each phase at equilibrium. 



\section{DEFINING THE NEXT GENERATION CAUCTIC-SIDE SOLVENT EXTRACTION SOLVENT COMPOSITION AND MODIFIED AQUEOUS FEEDS}

\subsection{INTRODUCTION}

The drive for an increased DF and CF resulted in a re-evaluation of the solvent composition of the BOBCalixC6 CSSX solvent. This chapter describes the determination of the preferred components of the NG-CSSX process, including the initial and modified solvent compositiosn and aqueous feeds. The ultimate goal was to replace the extractant, BOBCalixC6, with a more soluble ligand and to reduce the modifier concentration to promote better hydraulics by lowering the solvent density and viscosity (which is confirmed herein in Chapter 10). The more soluble extractant will allow a higher concentration to be used and enable a reduction in the modifier concentration to improve contactor hydraulics and potentially increase the rate of processing.

The aqueous feeds for scrubbing and stripping were also redefined in order to achieve the high DF required. The BOBCalixC6 CSSX process utilizes a nitrate swing to effect extraction and stripping ${ }^{7}$. The high nitrate concentrations in the salt waste drive extraction as a consequence of the formation of a [(BOBCalixC6)Cs $\left.{ }^{+}\right] \mathrm{NO}_{3}^{-}$complex in the organic phase, followed by the release of $\mathrm{CsNO}_{3}{ }^{-}$by the BOBCalixC6 complex to the low nitrate concentration aqueous strip phase. Since the nitrate concentration cannot be lowered below approximately $1 \mathrm{mM}$ without negatively affecting phase separation, any increases in extraction $D_{C s}$ values seen with higher extractant concentration are offset by higher stripping $D_{C s}$ values driven by the nitrate swing, thus resulting in no process advantage overall. A different scrub-strip system was needed to escape from nitrate swing by changing to an inextractable anion in stripping. Boric acid was chosen as being the most compatible with downstream borosilicate vitrification. The resulting aqueous flowsheet required a new anion suppressor, replacing the TOA of the BOBCalixC6 CSSX solvent. In order to be effective, the new suppressor was required to function under the reduced acidity of the redefined striping feed.

In the course of carrying out the initial phases of development in FY 2010, the initial expectations for the best extractant and suppressor were overturned, resulting in final selection of respectively MaxCalix extractant and DCiTG suppressor. The initially targeted components of the NG-CSSX solvent were the extractant BEHBCalix, the suppressor TOA, and the Modifier Cs-7SB. The nature of the aqueous feeds were initially defined as a sodium hydroxide scrub solution, which would enable removal of the nitrate anions and a weakly acidic boric acid strip solution at a concentration as high as $0.1-0.5 \mathrm{M}$. In the spring and summer of 2010, various issues arose that required an evaluation of these initially defined solvent 
components as well as the concentrations of the aqueous solutions. The results presented in this present chapter will be reported in chronological order according to the evolution of NG-CSSX process development.

\subsection{EXPERIMENTAL SECTION}

\subsubsection{Materials}

Solvent components were obtained from commercial sources and judged to be of adequate purity for use as received (Section 2.2.1). Reagents are given in Table 3.1. BOBCalixC6 was obtained from IBC Advanced Technologies. Cs-7SB and MaxCalix were obtained from Marshallton Research Laboratories. TOA was obtained from Aldrich, and Isopar ${ }^{\circledR} \mathrm{L}$ was obtained from ExxonMobil (Table 3.1). As described in detail elsewhere ${ }^{10}$, DCiTG, the active guanidine reagent in LIX $^{\circledR} 79$ (Cognis), was precipitated as the HC1 form from a LIX 79 sample supplied by Cognis. Solvents were prepared by weighing appropriate amounts of extractant, modifier, and suppressor into volumetric flasks and diluting with Isopar L to the mark.

The major and minor components of the waste tank simulants used for the experiments that are discussed in this chapter are given in Table 3.2. Further details on the preparation of the waste-tank simulants can be found in Chapter 2 of this report. 
Table 3.1 BOBCalixC6 CSSX and NG-CSSX solvent components

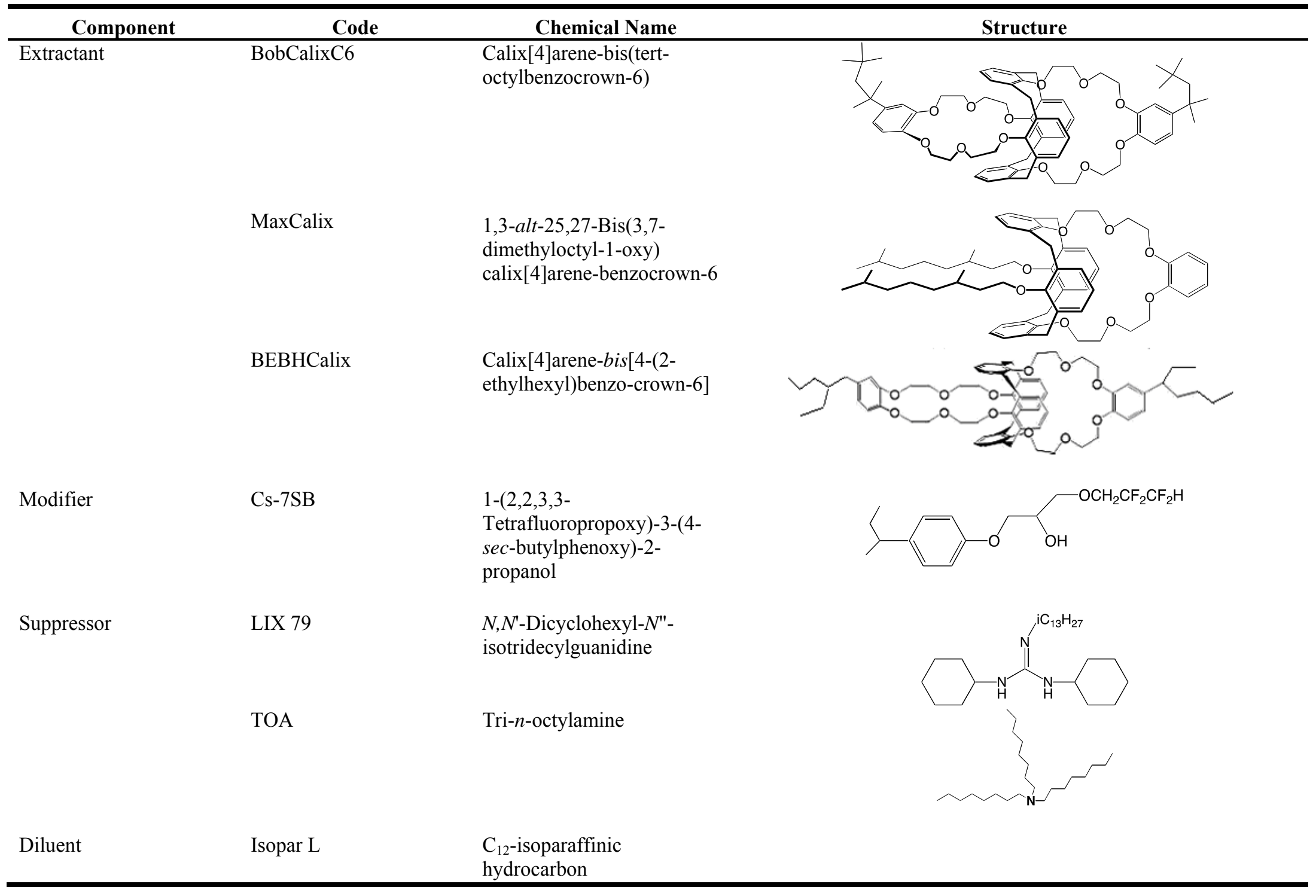


Table 3.2. Target compositions of aqueous tank-waste simulants

\begin{tabular}{|c|c|c|c|}
\hline Analyte or Species & $\begin{array}{c}\text { SRS-15 } \\
\mathrm{mol} / \mathrm{L}\end{array}$ & $\begin{array}{c}\mathrm{SRS}-45 \\
\mathrm{~mol} / \mathrm{L}\end{array}$ & $\begin{array}{c}\text { Hanford } \\
\mathrm{mol} / \mathrm{L}\end{array}$ \\
\hline \multicolumn{4}{|l|}{ Principal constituents: } \\
\hline $\mathrm{Al}$ as $\mathrm{Al}(\mathrm{OH})_{4}^{-}$ & 0.280 & 0.280 & 0.570 \\
\hline $\mathrm{Cl}^{-}$ & $2.4 \times 10^{-2}$ & $2.4 \times 10^{-2}$ & $9.4 \times 10^{-2}$ \\
\hline $\mathrm{CO}_{3}{ }^{2-}$ (TIC) & 0.150 & 0.150 & 0.367 \\
\hline $\mathrm{Cs}^{+}$(Total) & $1.4 \times 10^{-4}$ & $1.4 \times 10^{-4}$ & $5.82 \times 10^{-5}$ \\
\hline $\mathrm{K}^{+}$ & 0.015 & 0.045 & 0.174 \\
\hline $\mathrm{Na}^{+}$ & 5.60 & 5.60 & 6.00 \\
\hline $\mathrm{NO}_{2}^{-}$ & 0.500 & 0.500 & 1.17 \\
\hline $\mathrm{NO}_{3}^{-}$ & 2.03 & 2.06 & 1.90 \\
\hline $\mathrm{OH}^{-}$(Free) & 2.06 & 2.06 & 1.45 \\
\hline $\mathrm{SO}_{4}^{2-}$ & 0.140 & 0.140 & $3.22 \times 10^{-2}$ \\
\hline \multicolumn{4}{|l|}{ Minor inorganic constituents: } \\
\hline $\operatorname{Ag}(I)$ & $9.3 \times 10^{-8}$ & $9.3 \times 10^{-8}$ & \\
\hline Bi(III) & & & $5.6 \times 10^{-5}$ \\
\hline $\mathrm{Ca}^{2+}$ & & & $3.8 \times 10^{-4}$ \\
\hline $\mathrm{CrO}_{4}^{2-}$ & $1.4 \times 10^{-3}$ & $1.4 \times 10^{-3}$ & $1.0 \times 10^{-2}$ \\
\hline $\mathrm{Cu}(\mathrm{II})$ & $2.3 \times 10^{-5}$ & $2.3 \times 10^{-5}$ & \\
\hline $\mathrm{F}^{-}$ & $2.8 \times 10^{-2}$ & $2.8 \times 10^{-2}$ & $1.3 \times 10^{-2}$ \\
\hline $\mathrm{Fe}(\mathrm{III})$ & $2.6 \times 10^{-5}$ & $2.6 \times 10^{-5}$ & $1.0 \times 10^{-4}$ \\
\hline $\mathrm{Hg}(\mathrm{II})$ & $2.5 \times 10^{-7}$ & $2.5 \times 10^{-7}$ & $2.1 \times 10^{-9}$ \\
\hline $\mathrm{La}(\mathrm{III})$ & & & $1.0 \times 10^{-5}$ \\
\hline $\mathrm{MnO}_{4}^{-}$ & & & $2.1 \times 10^{-5}$ \\
\hline $\mathrm{MoO}_{4}^{2-}$ & $7.0 \times 10^{-5}$ & $7.0 \times 10^{-5}$ & \\
\hline $\mathrm{Ni}(\mathrm{II})$ & & & $3.3 \times 10^{-4}$ \\
\hline $\mathrm{NH}_{3}$ & $1.0 \times 10^{-3}$ & $1.0 \times 10^{-3}$ & \\
\hline $\mathrm{Pb}(\mathrm{II})$ & $1.0 \times 10^{-5}$ & $1.0 \times 10^{-5}$ & $9.7 \times 10^{-5}$ \\
\hline $\mathrm{Pd}(\mathrm{II})$ & $3.8 \times 10^{-6}$ & $3.8 \times 10^{-6}$ & \\
\hline $\mathrm{PO}_{4}^{3-}$ & $7.0 \times 10^{-3}$ & $7.0 \times 10^{-3}$ & $1.3 \times 10^{-2}$ \\
\hline $\mathrm{Rh}(\mathrm{III})$ & $2.0 \times 10^{-6}$ & $2.0 \times 10^{-6}$ & \\
\hline $\mathrm{Ru}(\mathrm{III})$ & $8.1 \times 10^{-6}$ & $8.1 \times 10^{-6}$ & \\
\hline $\mathrm{Si}(\mathrm{IV})$ & $3.0 \times 10^{-2}$ & $3.0 \times 10^{-2}$ & $2.3 \times 10^{-3}$ \\
\hline $\mathrm{Sn}(\mathrm{II})$ & $2.0 \times 10^{-5}$ & $2.0 \times 10^{-5}$ & \\
\hline $\mathrm{Sr}^{2+}$ & & & $4.5 \times 10^{-6}$ \\
\hline $\mathrm{Zn}(\mathrm{II})$ & $1.2 \times 10^{-4}$ & $1.2 \times 10^{-4}$ & \\
\hline $\mathrm{Zr}(\mathrm{IV})$ & & & $2.4 \times 10^{-5}$ \\
\hline \multicolumn{4}{|l|}{ Minor organic constituents: } \\
\hline Tri-n-butylphosphate (TBP) & $1.9 \times 10^{-6}$ & $1.9 \times 10^{-6}$ & \\
\hline Di- $n$-butylphosphate (DBP) & $1.2 \times 10^{-4}$ & $1.2 \times 10^{-4}$ & \\
\hline Mono- $n$-butylphosphate (MBP) & $1.6 \times 10^{-4}$ & $1.6 \times 10^{-4}$ & \\
\hline Formate $\left(\mathrm{HCO}_{2}^{-}\right)$ & $3.3 \times 10^{-2}$ & $3.3 \times 10^{-2}$ & \\
\hline Oxalate $\left(\mathrm{C}_{2} \mathrm{O}_{4}{ }^{2-}\right)$ & $8.0 \times 10^{-3}$ & $8.0 \times 10^{-3}$ & $1.0 \times 10^{-2}$ \\
\hline Trimethylamine & $1.7 \times 10^{-4}$ & $1.7 \times 10^{-4}$ & \\
\hline
\end{tabular}




\subsubsection{Methods}

\subsubsection{Solvent compositions}

The solvent compositions used in this chapter vary greatly between experiments. Hence, all solvent components and compositions will be detailed in either the description of the individual experiments or the tables relating to them.

\subsubsection{Aqueous solutions}

The SRS-15 simulant, designed to represent the average SRS tank-waste composition was prepared according to a method described previously ${ }^{12}$ (full composition given in Table 3.2). Appropriate amounts of reagent-grade chemicals were weighed into volumetric flasks in the order prescribed. Significant precipitate formed, which is considered normal, and the mixture was stirred for several hours and then allowed to settle. Tracer ${ }^{137} \mathrm{Cs}$ was added to the simulant for ESS tests $(4 \mu \mathrm{L}$ spike from a $0.05 \mathrm{mCi} / \mathrm{mL}$ stock, $\mathrm{CsCl}$ in $\mathrm{H}_{2} \mathrm{O}$, Eckert \& Ziegler Isotope Products Inc., formally Isotope Products, Burbank, CA).

Solutions of $\mathrm{NaOH}, \mathrm{HCl}$, and $\mathrm{H}_{3} \mathrm{BO}_{3}$ (Certified ACS, Fisher Scientific) were prepared using $>18 \mathrm{M} \Omega$ resistant reverse osmosis deionized water. All aqueous solutions were prepared using ACS reagent grade compounds (dried) and volumetric glassware.

\subsubsection{Cesium distribution ratios}

All $D_{C s}$ values in $\mathrm{ES}_{2} \mathrm{~S}_{3}$ tests using simulants were obtained in duplicate in the manner described in Chapter 2. The phases were contacted for 60 minutes for extraction and 45 minutes for scrubbing and stripping. All solutions unless otherwise noted were contacted inside of an air-box which was maintained at $22 \pm 3{ }^{\circ} \mathrm{C}$. After contacting, the tubes were placed in a centrifuge for 5 minutes at $3000 \mathrm{RPM}$ at experimental temperature. An appropriate aliquot of each phase was subsampled and counted using a Packard Cobra II Auto-Gamma counter. An appropriate aliquot of each phase was subsampled and counted using a Packard Cobra II Auto-Gamma counter. A spike of Cs-137 in the chloride form was added to the second and third aqueous strip solutions to maintain a high enough counting statistics and determine with reliability low $D_{\mathrm{Cs}}$. 


\subsection{RESULTS AND DISCUSSION}

\subsubsection{Preliminary Solvent Optimization}

\subsubsection{Selection of the Calixarene}

Two calixarene crown-ether alternatives to BOBCalixC6 were selected for testing (Table 3.1). Both have a higher solubility in the solvent matrix than BOBCalixC6 and are expected to have comparable extraction strengths. BEHBCalixC6 is very close in structure to BOBCalixC6 (in fact, they are isomers), wherein the tert-octyl chains are replaced with 2-ethylhexyl chains. The second calixarene, called MaxCalix, is a calix[4]arene monocrown-6 which was studied as part as the Fission Product Extraction process investigations ${ }^{13}$. The solvent compositions used in the following experiments are detailed in Table 3.3.

\subsubsection{Third-phase formation}

The critical test for these two calixarenes candidates is resistance to the formation of a third phase with varying temperature and aqueous feed. Table 3.3 summarizes the findings. As a comparison, results for the BOBCalixC6 CSSX solvent are also reported.

Table 3.3 Temperatures of third-phase formation ${ }^{a}$

\begin{tabular}{cccccccc}
\hline Simulant & BOBCalixC6 & \multicolumn{2}{c}{ BEHBCalix } & \multicolumn{3}{c}{ MaxCalix } \\
& $\mathbf{7} \mathbf{~ m M}$ & $\mathbf{7} \mathbf{~ m M}$ & $\mathbf{2 0} \mathbf{~ m M}$ & $\mathbf{5 0 ~ m M}^{\mathbf{b}}$ & $\mathbf{7} \mathbf{~ m M}$ & $\mathbf{2 0} \mathbf{~ m M}$ & $\mathbf{5 0} \mathbf{m M}^{\mathbf{b}}$ \\
\hline SRS-15 & $10^{\circ} \mathrm{C}$ & $9{ }^{\circ} \mathrm{C}$ & $16^{\circ} \mathrm{C}$ & $24{ }^{\circ} \mathrm{C}$ & $<5{ }^{\circ} \mathrm{C}$ & $7.5{ }^{\circ} \mathrm{C}$ & $6{ }^{\circ} \mathrm{C}$ \\
SRS-45 & $11{ }^{\circ} \mathrm{C}$ & $10^{\circ} \mathrm{C}$ & $19^{\circ} \mathrm{C}$ & $>25^{\circ} \mathrm{C}$ & $<5{ }^{\circ} \mathrm{C}$ & $7.5{ }^{\circ} \mathrm{C}$ & $12{ }^{\circ} \mathrm{C}$ \\
Hanford & $12.5^{\circ} \mathrm{C}$ & $10^{\circ} \mathrm{C}$ & $21.5^{\circ} \mathrm{C}$ & $>25{ }^{\circ} \mathrm{C}$ & $<5{ }^{\circ} \mathrm{C}$ & $9{ }^{\circ} \mathrm{C}$ & $14{ }^{\circ} \mathrm{C}$ \\
\hline
\end{tabular}

${ }^{a}$ Solvents contain the different calixarenes at the indicated concentrations, $0.75 \mathrm{M} \mathrm{Cs}-7 \mathrm{SB}$, and $3 \mathrm{mM}$ TOA in Isopar L. Temperatures mentioned in the table are the lowest temperatures for which no 3rd phase was detected.

${ }^{b}$ These solvents contained the calixarenes at the concentrations noted, $0.5 \mathrm{M} \mathrm{Cs-7SB}$ and $0.003 \mathrm{mM}$ LIX 79 guanidine in Isopar L.

The primary candidate, BEHBCalixC6 proved much more susceptible to third-phase formation than the competing extractant MaxCalix. Tests were run at $\mathrm{O}: \mathrm{A}=1: 5$ on contact with each of three waste simulants. The higher concentrations of BEHBCalix form visible third phases at room temperature, whereas MaxCalix resists third-phase formation to temperatures much lower than those anticipated under process conditions. For all subsequent studies, only MaxCalix was used (BOBCalixC6 was used for comparison purposes only).

The prospect of increasing the concentration of calixarene, due to improved solubility, leads naturally to a 
potential decrease in the concentration of the modifier, which would be expected to improve the hydraulic properties of the solvent. With a lower modifier concentration, however, a third phase may be expected to form at higher temperatures. Tests with $20 \mathrm{mM}$ MaxCalix, showed that, even for a concentration of Cs$7 \mathrm{SB}$ as low as $0.25 \mathrm{M}$, a third phase would not form for temperatures down to $10^{\circ} \mathrm{C}$ with the SRS feed and to $12{ }^{\circ} \mathrm{C}$ for the Hanford feed. As noted previously ${ }^{7}$, the third-phase susceptibility increases with potassium loading (SRS-15 $<$ SRS-45 $<$ Hanford).

\subsubsection{Selection of the suppressor}

Given the need to suppress effects averse to stripping ${ }^{10}$, it was important to settle upon the choice of either TOA or LIX 79 (Table 3.1) as the "suppressor". Initial tests ${ }^{10}$ had shown a very good stripping behavior with definite improvements over stripping in the BOBCalixC6 CSSX flowsheet when using TOA as suppressor with caustic scrub and boric acid strip solutions. An ESS experiment was run with MaxCalix and SRS-15 as the feed. Unexpectedly, and in contradiction to the previous ESS results ${ }^{10}$, the test showed that it was impossible to strip the solvent; these results were confirmed with a BOBcalixC6-based solvent (Table 3.4). Alternatively, when TOA was replaced by the guanidine derivative, both calixarene solvents exhibited excellent stripping. Further studies were undertaken to determine the origin of the discrepancy between these results and those reported previously ${ }^{3}$.

Table 3.4. Cesium distribution ratios comparison between TOA and DCiTG suppressors ${ }^{a, b}$

\begin{tabular}{lcccc}
\hline & \multicolumn{2}{c}{ 7 mM BOBCalixC6, 0.75 M Cs-7SB } & \multicolumn{2}{c}{ 20 mM MaxCalix, 0.5 M Cs-7SB } \\
& 3 mM TOA & 3 mM DCiTG & 3 mM TOA & 3 mM DCiTG \\
\hline Extraction & 14.3 & 14.0 & 25.5 & 25.2 \\
Scrub 1 & 2.40 & 2.14 & 2.85 & 2.42 \\
Scrub 2 & 2.61 & 2.05 & 2.71 & 2.29 \\
Strip 1 & 0.066 & $1.0 \times 10^{-3}$ & 0.094 & $1.0 \times 10^{-3}$ \\
Strip 2 & 0.386 & $6.0 \times 10^{-4}$ & 0.377 & $7.0 \times 10^{-4}$ \\
Strip 3 & 0.338 & $4.0 \times 10^{-4}$ & 0.416 & $4.0 \times 10^{-4}$ \\
\hline
\end{tabular}

${ }^{a}$ Aqueous phases: SRS- 15 on extraction, $0.1 \mathrm{M} \mathrm{NaOH}$ on scrubbing, and $10 \mathrm{mM} \mathrm{H}_{3} \mathrm{BO}_{3}$ on stripping, with respective O:A ratios of $1: 3,5: 1$, and $5: 1 . T=23 \pm 2{ }^{\circ} \mathrm{C}$

${ }^{b}$ The experimental data listed in this table is the result of single tube (nonduplicate) procedure.

It was determined that the spike of cesium-137 (as $\mathrm{HC} 1$ form) was the cause of the initial erroneous perception of the adequacy of TOA as a suppressor. In previous experiments, the spike came from a stock solution where the concentration of Cs-137 was $1 \mathrm{mCi} / \mathrm{mL}$ in $0.1 \mathrm{~N} \mathrm{HCl}$. The stock solution used for the current experiments was $10 \mathrm{mCi} / \mathrm{mL}$ in $0.1 \mathrm{~N} \mathrm{HCl}$. The dilution to get the same activity in the spike was therefore ten times greater, which also meant a much lower acidity. TOA is known to function as a 
suppressor when the strip solution is at least mildly acidic, preferably $\mathrm{pH} 3$ or lower. $^{7}$ A known minute amount of hydrochloric acid was added to the boric acid stripping solution, and stripping $D_{C s}$ values dropped drastically, demonstrating this effect. Thus, it was established that boric acid has insufficient acidity for TOA. The guanidine derivative was therefore chosen, and a solvent containing this suppressor along with Cs-7SB, and MaxCalix was tested for validation with the waste simulants from SRS.

\subsubsection{Selection of the Calixarene concentration}

The chosen calixarene, MaxCalix, being a calix[4]arene monocrown-6, has a binding strength for cesium that is expected to be comparable to, or only slightly lower, than that seen for BOBCalixCy. Monocrown calixarenes are also known to exhibit a better $\mathrm{Cs} / \mathrm{K}$ selectivity. With the ultimate goal of a higher throughput, tests were conducted with a concentration of MaxCalix about 3 times as high as that of BOBCalixC6 in the BOBCalixC6 CSSX process. The organic-phase complex contains one calixarene per cesium; hence, this ligand concentration increase should have had a linear impact on the $D_{C S}$; namely, the $D_{C s}$ values obtained with MaxCalix were expected to be around 40 with the SRS-15 simulant. The observed significantly lower distribution ratio confirms the weaker binding of calix-monocrown-6 for cesium. This effect is amplified by the fact that the solvent contained only $0.5 \mathrm{M}$ of the Cs-7SB modifier.

The behavior appears to be different when using the Hanford simulant. In that case, the solvent is nearly loaded with potassium. Using a solvent with a much larger ligand concentration affords a larger "cation capacity," combined with the fact that monocrown-caliarenes commonly have a better Cs/K selectivity ${ }^{3}$. Results are presented in Table 3.5.

Table 3.5. Batch ESS contacting results for two BOBCalixC6 CSSX solvents with SRS-15 and Hanford waste simulants ${ }^{a, b, c}$

\begin{tabular}{lcccc}
\hline \multicolumn{1}{c}{ Stage } & \multicolumn{2}{c}{ BOBCalix 7 mM } & \multicolumn{2}{c}{ MaxCalix 20 mM } \\
& SRS-15 & Hanford & SRS-15 & Hanford \\
\hline Extraction & 13.9 & 3.60 & 24.5 & 10.5 \\
Scrub 1 & 2.37 & 2.47 & 2.67 & 2.77 \\
Scrub 2 & 2.45 & 5.87 & 2.62 & 5.65 \\
Strip 1 & 0.064 & 3.06 & 0.093 & 5.20 \\
Strip 2 & 0.258 & 0.593 & 0.309 & 1.63 \\
Strip 3 & 0.179 & 0.216 & 0.297 & 0.558 \\
\hline
\end{tabular}

${ }^{a}$ The organic phase contained either BOBCalixC6 or MaxCalix. The BOBCalixC6 CSSX solvent containing $7 \mathrm{mM}$ BOBCalixC6 also contained $0.75 \mathrm{M} \mathrm{Cs}-7 \mathrm{SB}$ and $10 \mathrm{mM}$ TOA. The BOBCalixC6 CSSX solvent containing $20 \mathrm{mM}$ MaxCalix also consisted of $0.5 \mathrm{M} \mathrm{Cs}-7 \mathrm{SB}$ and $10 \mathrm{mM}$ TOA; both solvents used Isopar L diluent. The aqueous phase consisted of the SRS-15 or Hanford waste simulants. The scrub solution was $0.1 \mathrm{M} \mathrm{NaOH}$, and the strip solution contained $10 \mathrm{mM} \mathrm{H}_{3} \mathrm{BO}_{3}$. O:A ratios were 1:3 for extraction and 5:1 for scrubbing and stripping. Each contact was made at $25{ }^{\circ} \mathrm{C}$ and equilibrated for at least 30 minutes.

${ }^{b}$ Values reported in above table are not corrected for background. Actual $D_{\mathrm{Cs}}$ values for stripping are expected to be considerably lower

${ }^{c}$ The experimental data listed in this table is the result of single tube (non-duplicate) procedure. 
However, based on stage-efficiency estimations of current MCU performance in the range of $80-85 \%$, it has been considered that an even higher MaxCalix concentration in the solvent would provide a greater margin or robustness in reaching the target DF of $>40,000$ while being able to reduce O:A on extraction for a throughput gain ${ }^{3}$. Solvents where the concentration of MaxCalix was varied from $20 \mathrm{mM}$ to $50 \mathrm{mM}$ were tested (Tables 3.6.1 and 3.6.2) with two simulants from SRS (SRS-15 and SRS-45) where the only difference is the total concentration of potassium $(15 \mathrm{mM}$ versus $45 \mathrm{mM})$. As expected, the $D_{\mathrm{Cs}}$ increases with the concentration of ligand in all stages. The trend is essentially linear, as expected, with small differences that can be easily explained.

The reduction of the $D_{\mathrm{Cs}}$ on extraction with SRS-45 is due to the presence of potassium, which competes with cesium. This effect disappears with the scrub stages as potassium is quantitatively released. The first stripping stage might depart slightly from the strict linearity primarily due to the large release of cesium hydroxide into the aqueous solution. This increase in cesium salt in the aqueous phase yields higher $D_{\mathrm{Cs}}$, an effect that disappears in the subsequent strips. 
Table 3.6.1. Batch ESS contacting results one BOBCalixC6 CSSX solvent with differing concentrations of calixerene with SRS-15 ${ }^{a, b}$

\begin{tabular}{|c|c|c|c|c|c|c|}
\hline Stage & \multicolumn{2}{|c|}{20 mM MAX } & \multicolumn{2}{|c|}{$\begin{array}{c}\text { SRS-15 } \\
\text { 30 mM MAX }\end{array}$} & \multicolumn{2}{|c|}{50 mM MAX } \\
\hline Extraction & 25.7 & 25.6 & 37.5 & 37.3 & 56.8 & 56.8 \\
\hline Scrub 1 & 2.56 & 2.60 & 3.51 & 3.62 & 5.04 & 5.07 \\
\hline Strip 1 & $3.71 \times 10^{-4}$ & $3.66 \times 10^{-4}$ & $5.31 \times 10^{-4}$ & $6.30 \times 10^{-4}$ & $8.51 \times 10^{-4}$ & $9.25 \times 10^{-4}$ \\
\hline Strip 2 & $9.20 \mathrm{E} \times 10^{-5}$ & $2.62 \times 10^{-5}$ & $4.90 \times 10^{-5}$ & $5.19 \times 10^{-4}$ & $1.36 \times 10^{-4}$ & $1.54 \times 10^{-4}$ \\
\hline Strip 3 & $3.48 \times 10^{-5}$ & $9.52 \times 10^{-5}$ & $4.38 \times 10^{-5}$ & $4.69 \times 10^{-5}$ & $1.36 \times 10^{-4}$ & $1.40 \times 10^{-4}$ \\
\hline
\end{tabular}

${ }^{a}$ The organic phase consisted of one BOBCalixC6 CSSX solvent with three concentrations of calixerene. All solvents consisted of a concentration of MaxCalix, $0.50 \mathrm{M}$ Cs-7SB and $0.003 \mathrm{M}$ LIX 79 guanidine (DCiTG) in Isopar L diluent. The aqueous phase consisted of SRS-15 waste simulants. The scrub solution consisted of $0.1 \mathrm{M} \mathrm{NaOH}$ and the strip solutions consisted of $100 \mathrm{mM} \mathrm{H}_{3} \mathrm{BO}_{3}$. O:A ratios were 1:3 for extraction and 5:1 for scrubbing and stripping. Each contact was made at 25 ${ }^{\circ} \mathrm{C}$ and equilibrated for at least 30 minutes.

${ }^{b}$ All values are background corrected. Values obtained for $20 \mathrm{mM}$ and $30 \mathrm{mM}$ MAX were background corrected with background counts obtained as an average from later experiments.

Table 3.6.2. Batch ESS contacting results one BOBCalixC6 CSSX solvent with differing concentrations of calixerene with SRS-45

\begin{tabular}{|c|c|c|c|c|c|c|}
\hline \multirow{2}{*}{$\frac{\text { Stage }}{\text { Extraction }}$} & \multicolumn{2}{|c|}{20 mM MAX } & \multicolumn{2}{|c|}{$\begin{array}{c}\text { SRS-45 } \\
\text { 30 } \mathrm{mM} \mathrm{MAX}\end{array}$} & \multicolumn{2}{|c|}{50 mM MAX } \\
\hline & 21.2 & 21.3 & 31.5 & 31.4 & 48.1 & 48.1 \\
\hline Scrub 1 & 2.50 & 2.50 & 3.50 & 3.55 & 5.09 & 5.16 \\
\hline Scrub 2 & 2.42 & 2.42 & 3.27 & 3.26 & 4.37 & 4.28 \\
\hline Strip 1 & $3.89 \times 10^{-4}$ & $3.66 \times 10^{-4}$ & $6.35 \times 10^{-4}$ & $6.68 \times 10^{-4}$ & $1.14 \times 10^{-3}$ & $1.12 \times 10^{-3}$ \\
\hline Strip 2 & $9.23 \times 10^{-5}$ & $4.32 \times 10^{-5}$ & $5.21 \times 10^{-4}$ & $5.09 \times 10^{-5}$ & $1.76 \times 10^{-4}$ & $1.62 \times 10^{-4}$ \\
\hline Strip 3 & $8.45 \times 10^{-5}$ & $8.09 \times 10^{-6}$ & $4.52 \times 10^{-5}$ & $4.62 \times 10^{-5}$ & $1.64 \times 10^{-4}$ & $2.01 \times 10^{-4}$ \\
\hline
\end{tabular}

${ }^{a}$ The organic phase consisted of one BOBCalixC6 CSSX solvent with three concentrations of calixerene. All solvents consisted of a concentration of MaxCalix, 0.50 M Cs7SB and 0.003 M LIX 79 guanidine (DCiTG) in Isopar L diluent. The aqueous phase consisted of SRS-45 waste simulants. The scrub solution consisted of $0.1 \mathrm{M}$ NaOH and the strip solutions consisted of $100 \mathrm{mM} \mathrm{H}_{3} \mathrm{BO}_{3}$. O:A ratios were 1:3 for extraction and 5:1 for scrubbing and stripping. Each contact was made at $25{ }^{\circ} \mathrm{C}$ and equilibrated for at least 30 minutes.

${ }^{b}$ All values are background corrected. Values obtained for $20 \mathrm{mM}$ and $30 \mathrm{mM}$ MAX were background corrected with background counts obtained as an average from later experiments. 


\subsubsection{Selection of the modifier concentration}

At a given feed composition, the $D_{C s}$ obtained upon extraction are primarily influenced by the concentration of the calixarene and the concentration of the modifier. While the $D_{C s}$ vary linearly with the calixarene concentration, the impact of the modifier is less prominent. Lowering the concentration of modifier in the system decreases the viscosity of the solvent and yields a higher throughput. Two lower concentrations of modifier Cs-7SB were compared to the results obtained with the $0.75 \mathrm{M}$ concentration used in the BOBCalixC6 CSSX process (Table 3.7). Testing showed reducing the concentration by $66 \%$ to $0.25 \mathrm{M}$ is too drastic and impacts negatively the $D_{C S}$ on extraction, while the solvent containing $0.5 \mathrm{M}$ of modifier provides adequate results.

Table 3.7. Batch ESS contacting results for two BOBCalixC6 CSSX solvents with SRS-45 waste simulant ${ }^{a b}$

\begin{tabular}{lcccccc}
\hline \multicolumn{1}{c}{ Stage } & \multicolumn{2}{c}{$\begin{array}{c}\mathbf{0 . 2 5 M} \text { Cs-7SB } \\
\text { 3 mM DCiTG }\end{array}$} & \multicolumn{2}{c}{$\mathbf{0 . 5 0 M}$ Cs-7SB } & \multicolumn{2}{c}{$\mathbf{0 . 7 5}$ M Cs-7SB } \\
& \multicolumn{2}{c}{$\mathbf{3 ~ m M ~ D C i T G ~}$} & \multicolumn{2}{c}{$\mathbf{1 ~ m M ~ D C i T G ~}$} \\
\hline Extraction & 11.6 & 11.6 & 21.1 & 20.7 & 25.2 & 25.6 \\
Scrub 1 & 1.04 & 1.03 & 2.50 & 2.52 & 3.84 & 3.60 \\
Scrub 2 & 1.02 & 1.03 & 2.42 & 2.42 & 3.93 & 3.97 \\
Strip 1 & $2.51 \times 10^{-4}$ & $2.80 \times 10^{-4}$ & $4.75 \times 10^{-4}$ & $4.53 \times 10^{-4}$ & $1.03 \times 10^{-3}$ & $1.13 \times 10^{-3}$ \\
Strip 2 & $4.26 \times 10^{-4}$ & $3.74 \times 10^{-4}$ & $5.10 \times 10^{-4}$ & $4.47 \times 10^{-4}$ & $4.74 \times 10^{-4}$ & $4.42 \times 10^{-4}$ \\
Strip 3 & $4.37 \times 10^{-4}$ & $4.22 \times 10^{-4}$ & $4.67 \times 10^{-4}$ & $3.96 \times 10^{-4}$ & $5.69 \times 10^{-4}$ & $4.53 \times 10^{-4}$ \\
\hline
\end{tabular}

${ }^{\mathrm{a}}$ The organic phase consisted of $0.020 \mathrm{M}$ MaxCalix with varied suppressor (DCiTG) and modifier (Cs-7SB) concentrations in Isopar L diluent. The aqueous phase consisted of the SRS-45 waste simulant. The scrub solution was $0.1 \mathrm{M} \mathrm{NaOH}$ and the strip solution was $100 \mathrm{mM} \mathrm{H}_{3} \mathrm{BO}_{3}$. O:A ratios were 1:3 for extraction and 5:1 for scrubbing and stripping. Each contact was made at $25^{\circ} \mathrm{C}$ and equilibrated for at least 30 minutes.

${ }^{\mathrm{b}}$ Values reported in above table are not corrected for background. Actual $D_{C s}$ values for stripping are expected to be considerably lower.

\subsubsection{Selection of the suppressor concentration}

The selection of the suppressor concentration is based on the robustness of the solvent to common organophilic impurities, such as sodium dodecyl sulfate (SDS). Soap residues were determined to be the cause of poor stripping performance when BOBCalixC6 CSSX was originally developed ${ }^{14}$ and the impact of deliberate addition of SDS at different concentrations has been systematically studied to make sure that the concentration of the suppressor is sufficient to overcome the presence of SDS at concentrations between $0.01 \mathrm{mM}$ and $0.1 \mathrm{mM}, 1 \mathrm{mM}$ being an upper limit unlikely to be encountered in real systems. Results presented in Table 3.8 confirm the inability of TOA to provide an effective stripping in the presence of SDS. 
Table 3.8. Batch ESS contacting results for one BOBCalixC6 CSSX solvent (containing TOA) with SRS-15 simulant containing three different concentrations of SDS impurity ${ }^{a, b}$

\begin{tabular}{lcccc}
\hline \multicolumn{1}{c}{ Stage } & NO SDS & $\mathbf{1 . 0} \times \mathbf{1 0}^{-\mathbf{4}} \mathbf{M}$ SDS & $\mathbf{1 . 0} \times \mathbf{1 0}^{-4} \mathbf{M}$ SDS & $\mathbf{1 . 0} \times \mathbf{1 0}^{-3} \mathbf{M ~ S D S}$ \\
\hline Extraction & 26.3 & 25.9 & 27.9 & 40.5 \\
Scrub 1 & 2.83 & 3.04 & 4.73 & 62.8 \\
Scrub 2 & 3.04 & 3.28 & 8.36 & 209 \\
Strip 1 & 0.0966 & 0.124 & 0.948 & 400 \\
Strip 2 & 0.278 & 0.366 & 1.31 & 324 \\
Strip 3 & 0.136 & 0.225 & 0.938 & 300 \\
\hline
\end{tabular}

${ }^{a}$ The organic phase consisted of 0.020 M MaxCalix, 0.50 M Cs-7SB modifier, and 0.003 M TOA in Isopar L diluent. The aqueous phase consisted of the SRS- 15 waste simulant at four concentrations of SDS. The scrub solution was $0.1 \mathrm{M} \mathrm{NaOH}$ and the strip was a solution of $\mathrm{H}_{3} \mathrm{BO}_{3} / \mathrm{HCl}, 0.001 \mathrm{M}$ and $0.0001 \mathrm{M}$ respectively. O:A ratios were 1:3 for extraction and 5:1 for scrubbing and stripping. Each contact was made at $25^{\circ} \mathrm{C}$ and equilibrated for at least 30 minutes.

${ }^{b}$ Values reported in above table are not corrected for background. Actual $D_{C S}$ values for stripping are expected to be considerably lower.

The recommended switch from TOA to DCiTG, while keeping a $3 \mathrm{mM}$ concentration of the new suppressor, provides excellent results for the stripping steps, for all three simulants tested (Tables 3.9 through 3.11). The Hanford simulant spiked with the highest SDS concentrations showed unacceptable stripping values. An increase in the DCiTG concentration may mitigate higher SDS concentrations in Hanford simulants and also increase process robustness.

Table 3.9. Batch ESS contacting results for one BOBCalixC6 CSSX solvent (containing DCiTG) with SRS-15 simulant containing four concentrations of SDS impurity ${ }^{a, b}$

\begin{tabular}{|c|c|c|c|c|}
\hline Stage & NO SDS & $1.0 \times 10^{-5}$ M SDS & $1.0 \times 10^{-4}$ M SDS & $1.0 \times 10^{-3}$ M SDS \\
\hline Extraction & 27.0 & 26.6 & 27.5 & 38.3 \\
\hline Scrub 1 & 2.60 & 2.73 & 4.11 & 26.7 \\
\hline Scrub 2 & 2.55 & 2.59 & 5.06 & 51.6 \\
\hline Strip 1 & $1.25 \times 10^{-3}$ & $1.32 \times 10^{-3}$ & $2.57 \times 10^{-3}$ & $9.77 \times 10^{-3}$ \\
\hline Strip 2 & $5.95 \times 10^{-4}$ & $6.41 \times 10^{-4}$ & $8.09 \times 10^{-4}$ & $1.55 \times 10^{-2}$ \\
\hline Strip 3 & $5.31 \times 10^{-4}$ & $4.84 \times 10^{-4}$ & $5.89 \times 10^{-4}$ & $3.14 \times 10^{-3}$ \\
\hline
\end{tabular}

${ }^{a}$ The organic phase consisted of 0.020M MaxCalix, 0.50M Cs-7SB modifier, and 0.003M LIX 79 guanidine (DCiTG) in Isopar L diluent. The aqueous phase consisted of the SRS-15 waste simulant at four concentrations of SDS. The scrub was $0.1 \mathrm{M} \mathrm{NaOH}$ and the strip was $10 \mathrm{mM} \mathrm{H}_{3} \mathrm{BO}_{3}$. O:A ratios were 1.:3 for extraction and 5:1 for scrubbing and stripping. Each contact was made at $25^{\circ} \mathrm{C}$ and equilibrated for at least 30 minutes.

${ }^{b}$ Values reported in above table are not corrected for background. Actual $D_{C s}$ values for stripping are expected to be considerably lower. 
Table 3.10. Batch ESS contacting for one BOBCalixC6 CSSX solvent (containing DCiTG) with Hanford simulant containing four concentrations of SDS impurity ${ }^{a, b}$

\begin{tabular}{llccc}
\hline \multicolumn{1}{c}{ Stage } & NO SDS & $\mathbf{1 . 0} \times \mathbf{1 0}^{-\mathbf{5}} \mathbf{M ~ S D S}$ & $\mathbf{1 . 0} \times \mathbf{1 0}^{-\mathbf{4}} \mathbf{M} \mathbf{S D S}$ & $\mathbf{1 . 0} \times \mathbf{1 0}^{-\mathbf{3}} \mathbf{M} \mathbf{S D S}$ \\
\hline Extraction & 10.6 & 10.6 & 10.8 & 11.9 \\
Scrub 1 & 2.78 & 2.92 & 3.32 & 13.0 \\
Scrub 2 & 4.43 & 4.71 & 6.60 & 5.36 \\
Strip 1 & $1.16 \times 10^{-3}$ & $1.46 \times 10^{-3}$ & $2.18 \times 10^{-3}$ & 5.37 \\
Strip 2 & $7.54 \times 10^{-4}$ & $6.25 \times 10^{-4}$ & $7.42 \times 10^{-4}$ & 26.0 \\
Strip 3 & $6.39 \times 10^{-4}$ & $4.59 \times 10^{-4}$ & $6.67 \times 10^{-4}$ & 55.6 \\
\hline
\end{tabular}

${ }^{\mathrm{a}}$ The organic phase consisted of 0.020 M MaxCalix, 0.50 M Cs-7SB modifier, and 0.0003 M LIX 79 DCiTG in Isopar L diluent. The aqueous phase consisted of the Hanford waste simulant at four concentrations of SDS. The scrub was $0.1 \mathrm{M} \mathrm{NaOH}$ and the strip was $10 \mathrm{mM} \mathrm{H}_{3} \mathrm{BO}_{3}$. O:A ratios were 1:3 for extraction and 5:1 for scrubbing and stripping. Each contact was made at $25^{\circ} \mathrm{C}$ and equilibrated for at least 30 minutes.

${ }^{b}$ Values reported in above table are not corrected for background. Actual $D_{C s}$ values for stripping are expected to be considerably lower.

Table 3.11. Batch ESS contacting results for robustness of the NG-CSSX Solvent. Scrub with $0.1 \mathrm{~N} \mathrm{NaOH}$

\begin{tabular}{|c|c|c|c|c|}
\hline & No SDS & $1.0 \times 10^{-5} \mathrm{M}$ SDS & $1.0 \times 10^{-4} M$ SDS & $1.0 \times 10^{-3} \mathrm{M}$ SDS \\
\hline Extraction & 54.6 & 56.5 & 56.9 & 68.1 \\
\hline Scrub 1 & 5.05 & 5.16 & 6.02 & 19.6 \\
\hline Scrub 2 & 4.40 & 4.52 & 6.39 & 37.3 \\
\hline Strip 1 & $8.92 \times 10^{-4}$ & $9.35 \times 10^{-4}$ & $1.08 \times 10^{-3}$ & $8.66 \times 10^{-3}$ \\
\hline Strip 2 & $1.74 \times 10^{-4}$ & $3.13 \times 10^{-4}$ & $2.31 \times 10^{-4}$ & $7.69 \times 10^{-4}$ \\
\hline Strip 3 & $3.30 \times 10^{-4}$ & $2.05 \times 10^{-4}$ & $2.60 \times 10^{-4}$ & $5.30 \times 10^{-4}$ \\
\hline \multicolumn{5}{|c|}{$\begin{array}{l}\text { The organic phase consisted of one BOBCalixC6 CSSX solvent containing } 50 \mathrm{mM} \text { MaxCalix, } 0.50 \mathrm{M} \mathrm{Cs}-7 \mathrm{SB} \text { and } 0.003 \\
\text { M LIX } 79 \text { guanidine (DCiTG) in Isopar L diluent. The aqueous phase consisted of SRS-15 and SRS- } 45 \text { waste simulants containg } \\
\text { different concentrations of SDS impurity (noted in table above). The scrub consisted of } 100 \mathrm{mM} \mathrm{NaOH} \text {. The strip solutions } \\
\text { consisted of } 100 \mathrm{mM} \mathrm{H}_{3} \mathrm{BO}_{3} \text {. O:A ratios were } 1: 3 \text { for extraction and } 5: 1 \text { for scrubbing and stripping. Each contact was made at } \\
25{ }^{\circ} \mathrm{C} \text { and equilibrated for at least } 30 \text { minutes. }\end{array}$} \\
\hline
\end{tabular}

\subsubsection{Aqueous Composition for Scrub and Strip Stages}

\subsubsection{Selection of scrub component and concentration}

The transition from the BOBCalixC6 CSSX process to the NG-CSSX process was tested in several steps when it came to the definition of the aqueous phases for the scrub and strip stages. The BOBCalixC6 CSSX process uses nitric acid at different concentrations for both the scrub and strip aqueous feeds, but nitric acid as a scrub solution was ruled out early on due to the noticeable carry-over of nitrate from the second scrub into the first strip, which lead to unacceptably high distribution ratios for the first strip stage. A switch to a caustic scrub was implemented which ensured the release of potassium from the NG-CSSX solvent while retaining the majority of the cesium, which was released during the stripping stages. From the potential options for a scrub solution, an obvious choice was sodium hydroxide, due to its simplicity, 
compatibility with the feed and the low affinity the NG-CSSX solvent has for sodium.

As a preliminary test, three concentrations of sodium hydroxide anticipated to provide adequate $D_{C s}$ (i.e., greater than 1) were used with the solvent containing $30 \mathrm{mM}$ of MaxCalix (Table 3.12). This test was expended when the concentration of $50 \mathrm{mM}$ of MaxCalix was adopted (Table 3.13). It can be seen that deionized water, or $10 \mathrm{mM} \mathrm{NaOH}$, would not be adequate as scrub solutions due to the low $D_{C s}$. The selection from the other three concentrations $(25,50$, and $100 \mathrm{mM})$ was based on the appearance of the aqueous phase post-contact. The higher concentrations of sodium hydroxide tended to have a cloudy appearance (Table 3.14), leaving $25 \mathrm{mM}$ of $\mathrm{NaOH}$ as the preferred scrub solution. 
Table 3.12. Batch ESS contacting results for one BOBCalixC6 CSSX solvent with different waste simulants and scrubbing solutions ${ }^{a, b}$

\begin{tabular}{|c|c|c|c|c|c|c|c|c|}
\hline \multirow[b]{2}{*}{ Stage } & \multicolumn{3}{|c|}{ SRS-15 } & \multicolumn{5}{|c|}{ SRS-45 } \\
\hline & \multicolumn{2}{|c|}{$50 \mathrm{mM} \mathrm{NaOH}$} & \multicolumn{2}{|c|}{$100 \mathrm{mM} \mathrm{NaOH}$} & \multicolumn{2}{|c|}{$50 \mathrm{mM} \mathrm{NaOH}$} & \multicolumn{2}{|c|}{$100 \mathrm{mM} \mathrm{NaOH}$} \\
\hline Extraction & 37.2 & 38.7 & 37.5 & 37.3 & 30.9 & 30.8 & 31.5 & 31.4 \\
\hline Scrub1 & 2.66 & 2.69 & 3.51 & 3.62 & 2.82 & 2.81 & 3.50 & 3.55 \\
\hline Scrub 2 & 2.02 & 2.02 & 3.22 & 3.33 & 2.11 & 2.16 & 3.27 & 3.26 \\
\hline Strip 1 & $6.68 \times 10^{-4}$ & $6.28 \times 10^{-4}$ & $5.31 \times 10^{-4}$ & $6.30 \times 10^{-4}$ & $6.16 \times 10^{-4}$ & $5.84 \times 10^{-4}$ & $6.35 \times 10^{-4}$ & $6.68 \times 10^{-4}$ \\
\hline Strip 2 & $4.73 \times 10^{-4}$ & $4.46 \times 10^{-4}$ & $4.90 \times 10^{-4}$ & $5.19 \times 10^{-4}$ & $4.56 \times 10^{-4}$ & $4.31 \times 10^{-4}$ & $5.21 \times 10^{-4}$ & $5.09 \times 10^{-4}$ \\
\hline Strip 3 & $4.83 \times 10^{-4}$ & $4.65 \times 10^{-4}$ & $4.38 \times 10^{-4}$ & $4.69 \times 10^{-4}$ & $4.51 \times 10^{-4}$ & $4.43 \times 10^{-4}$ & $4.52 \times 10^{-4}$ & $4.62 \times 10^{-4}$ \\
\hline
\end{tabular}

${ }^{a}$ The organic phase consisted of $0.030 \mathrm{M}$ MaxCalix, $0.50 \mathrm{M}$ Cs-7SB modifier, and 0.003 M LIX 79 guanidine (DCiTG) Isopar L diluent. The aqueous phase consisted of the SRS-15 and SRS-45 waste simulants. The scrub solutions consisted of either $50 \mathrm{mM}$ or $100 \mathrm{mM} \mathrm{NaOH}$. The strip solution consisted of $100 \mathrm{mM} \mathrm{\textrm {H } _ { 3 } \mathrm { BO }}{ }_{3}$. O:A ratios were 1:3 for extraction and 5:1 for scrubbing and stripping. Each contact was made at $25{ }^{\circ} \mathrm{C}$ and equilibrated for at least 30 minutes.

${ }^{b}$ Values reported in above table are not corrected for background. Actual $D_{C s}$ values for stripping are expected to be considerably lower.

Table 3.13. Batch ESS contacting results one BOBCalixC6 CSSX solvent with differing concentrations of scrub (NaOH) solution ${ }^{a, b}$

\begin{tabular}{|c|c|c|c|c|c|c|c|c|c|c|}
\hline \multirow[t]{2}{*}{ Stage } & \multicolumn{5}{|c|}{ SRS-15 } & \multicolumn{5}{|c|}{ SRS-45 } \\
\hline & DI Water & $10 \mathrm{mM} \mathrm{NaOH}$ & $25 \mathrm{mM} \mathrm{NaOH}$ & $50 \mathrm{mM}$ NaOH & $100 \mathrm{mM} \mathrm{NaOH}$ & DI Water & $10 \mathrm{mM} \mathrm{NaOH}$ & $25 \mathrm{mM} \mathrm{NaOH}$ & $50 \mathrm{mM} \mathrm{NaOH}$ & $100 \mathrm{mM} \mathrm{NaOH}$ \\
\hline Extraction & 55.8 & 55.9 & 53.7 & 56.7 & 56.8 & 47.8 & 47.6 & 48.2 & 48.1 & 48.1 \\
\hline Scrub 1 & 2.94 & 3.15 & 3.56 & 4.05 & 5.06 & 3.50 & 3.70 & 3.95 & 4.30 & 5.13 \\
\hline Scrub 2 & 0.329 & 1.07 & 1.76 & 2.87 & 4.51 & 0.71 & 1.35 & 1.51 & 2.86 & 4.33 \\
\hline Strip 1 & $6.75 \times 10^{-4}$ & $7.11 \times 10^{-4}$ & $6.88 \times 10^{-4}$ & $7.64 \times 10^{-4}$ & $8.88 \times 10^{-4}$ & $8.27 \times 10^{-4}$ & $8.30 \times 10^{-4}$ & $8.04 \times 10^{-4}$ & $9.25 \times 10^{-4}$ & $1.13 \times 10^{-3}$ \\
\hline Strip 2 & $2.33 \times 10^{-4}$ & $3.28 \times 10^{-4}$ & $1.53 \times 10^{-4}$ & $1.25 \times 10^{-4}$ & $1.45 \times 10^{-4}$ & $3.22 \times 10^{-4}$ & $2.27 \times 10^{-4}$ & $2.20 \times 10^{-4}$ & $1.98 \times 10^{-4}$ & $1.69 \times 10^{-4}$ \\
\hline Strip 3 & $4.64 \times 10^{-4}$ & $2.12 \times 10^{-4}$ & $2.19 \times 10^{-4}$ & $1.49 \times 10^{-4}$ & $1.38 \times 10^{-4}$ & $1.86 \times 10^{-4}$ & $1.46 \times 10^{-4}$ & $2.35 \times 10^{-4}$ & $2.22 \times 10^{-4}$ & $1.83 \times 10^{-4}$ \\
\hline
\end{tabular}

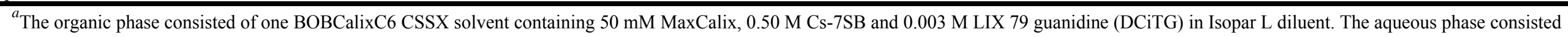

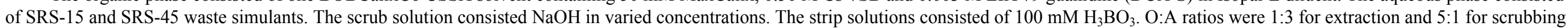
and stripping. Each contact was made at $25{ }^{\circ} \mathrm{C}$ and equilibrated for at least 30 minutes.

${ }^{b}$ All values have been background corrected and are averages of experimental duplicates. 
Table 3.14. Relative clarity of the aqueous phase at each stage of the ESS contact ${ }^{a}$

\begin{tabular}{|c|c|c|c|c|c|c|}
\hline \multirow[t]{2}{*}{ Stage } & \multicolumn{3}{|c|}{ SRS-15 } & \multicolumn{3}{|c|}{ SRS-45 } \\
\hline & $\begin{array}{l}25 \mathrm{mM} \\
\mathrm{NaOH}\end{array}$ & $\begin{array}{l}50 \mathrm{mM} \\
\mathrm{NaOH}\end{array}$ & $\begin{array}{c}100 \mathrm{mM} \\
\mathrm{NaOH}\end{array}$ & $\begin{array}{l}25 \mathrm{mM} \\
\mathrm{NaOH}\end{array}$ & $\begin{array}{l}50 \mathrm{mM} \\
\mathrm{NaOH}\end{array}$ & $\begin{array}{c}100 \mathrm{mM} \\
\mathrm{NaOH}\end{array}$ \\
\hline Extraction & Clear & Clear & Clear & Clear & Clear & Clear \\
\hline Scrub 1 & Sl. Hazy & Hazy & Hazy & Hazy & Sl. Milky & Milky \\
\hline Scrub 2 & Clear & Hazy & Hazy & Clear & Hazy & Sl. Milky \\
\hline Strip 1 & Clear & Clear & Clear & Clear & Clear & Clear \\
\hline Strip 2 & Clear & Clear & Clear & Clear & Clear & Clear \\
\hline Strip 3 & Clear & Clear & Clear & Clear & Clear & Clear \\
\hline
\end{tabular}

${ }^{a}$ The organic phase consisted of one BOBCalixC6 CSSX solvent containing $50 \mathrm{mM}$ MaxCalix, $0.50 \mathrm{M}$ Cs-7SB and 0.003 M LIX 79 guanidine (DCiTG) in Isopar L diluent. The aqueous phase consisted of SRS-15 and SRS-45 waste simulants. The scrub solution comprised $\mathrm{NaOH}$ in varied concentrations. The strip solutions consisted of $100 \mathrm{mM} \mathrm{H}_{3} \mathrm{BO}_{3}$. O:A ratios were 1:3 for extraction and 5:1 for scrubbing and stripping. Each contact was made at $25^{\circ} \mathrm{C}$ and equilibrated for at least 30 minutes.

\subsubsection{Selection of stripping component and concentration}

With a caustic scrub stage and the complete metathesis of nitrate for hydroxide, removal of the cesium, in theory, only requires acid titration of the $\mathrm{OH}^{-}$ions, whilst presenting the cesium with a highly hydrophilic alternative anion. Boric acid was the natural choice, being a weak acid and having a very hydrophilic conjugate base, the $\mathrm{H}_{3} \mathrm{BO}_{3}$ anion. Borate is also a very suitable anion for downstream processing of the cesium; the vitrification process in borosilicate glass.

To determine the most suitable concentration of boric acid, multiple concentrations were tested (10-500 $\mathrm{mM}$ ), and the stripping behavior was monitored. It can be seen in Table 3.15 (below) that stripping does not improve at boric acid concentrations higher than $100 \mathrm{mM}$. This seemed like an appropriate concentration, when compared to $10 \mathrm{mM}$, as the assumed increased ionic strength was thought to aid in phase disengatement. In later testing, this was found to be the inverse and interaction between the DCiTG and the boric acid increased with increasing concentration and resulted in third-phase formation (see also Chapter 8). 
Table 3.15. Batch ESS contacting results for NG-CSSX solvent with SRS-45 and SRS-15 simulants using three stripping solutions ${ }^{a, b}$

\begin{tabular}{|c|c|c|c|c|c|c|c|c|}
\hline \multirow{2}{*}{$\begin{array}{c}\text { Stage } \\
\text { Extraction }\end{array}$} & \multicolumn{6}{|c|}{ SRS-45 } & \multicolumn{2}{|c|}{$\begin{array}{c}\text { SRS-15 } \\
100 \mathrm{mM} \mathrm{H}_{3} \mathrm{BO}_{3} \\
\end{array}$} \\
\hline & 21.2 & 21.3 & 21.1 & 20.7 & 20.9 & 21.3 & 25.54 & 25.45 \\
\hline Scrub 1 & 2.50 & 2.44 & 2.50 & 2.52 & 2.50 & 2.50 & 2.56 & 2.59 \\
\hline Scrub 2 & 2.44 & 2.44 & 2.42 & 2.42 & 2.39 & 2.39 & 2.34 & 2.34 \\
\hline Strip 1 & $8.85 \times 10^{-4}$ & $8.67 \times 10^{-4}$ & $4.75 \times 10^{-4}$ & $4.53 \times 10^{-4}$ & $4.62 \times 10^{-4}$ & $4.88 \times 10^{-4}$ & $4.53 \times 10^{-4}$ & $4.48 \times 10^{-4}$ \\
\hline Strip 2 & $5.64 \times 10^{-4}$ & $4.76 \times 10^{-4}$ & $5.10 \times 10^{-4}$ & $4.47 \times 10^{-4}$ & $4.84 \times 10^{-4}$ & $5.77 \times 10^{-4}$ & $5.09 \times 10^{-4}$ & $4.12 \times 10^{-4}$ \\
\hline Strip 3 & $4.55 \times 10^{-4}$ & $5.76 \times 10^{-4}$ & $4.67 \times 10^{-4}$ & $3.96 \times 10^{-4}$ & $4.57 \times 10^{-4}$ & $4.70 \times 10^{-4}$ & $4.14 \times 10^{-4}$ & $4.67 \times 10^{-4}$ \\
\hline
\end{tabular}

${ }^{a}$ The organic phase consisted of 0.020 M MaxCalix, 0.50 M Cs-7SB modifier, and 0.003 LIX 79 guanidine (DCiTG) Isopar L diluent. The aqueous phase consisted of the SRS-45 and SRS-15 waste simulants. The scrub solution was $0.1 \mathrm{M} \mathrm{NaOH}$ and the strip solutions consisted of $\mathrm{H}_{3} \mathrm{BO}_{3}$ in varied concentrations. O:A ratios were 1:3 for extraction and 5:1 for scrubbing and stripping. Each contact was made at $25^{\circ} \mathrm{C}$ and equilibrated for at least 30 minutes.

${ }^{b}$ Values reported in above table are not corrected for background. Actual $D_{C s}$ values for stripping are expected to be considerably lower.

With the implementation of improved O:A ratios (discussed in the following section) and increased MaxCalix concentration (to $50 \mathrm{mM}$ ) in the solvent, it became necessary to re-test how the stripping solution performs. The concentration of $\mathrm{Cs}^{+}$was increased from $0.14 \mathrm{mM}$ to $0.6 \mathrm{mM}$ in the SRS waste simulant and the effect on the stripping was monitored. The boric acid concentration was also varied retaining the higher $\mathrm{Cs}^{+}$concentration. From Table 3.16 it can be observed that as the concentration of the boric acid is increased, the stripping during the first stage is inversely proportional to the $D_{C s}$ value. However by the third stage of stripping with the SRS-15 simulant, $10 \mathrm{mM}$ boric acid performs the same as the $50 \mathrm{mM}$ boric acid. Extraction and scrubbing $D_{C s}$ values appear the same across all representative sets.

Table 3.16. Cesium distribution ratios from ESS experiments with SRS-15 waste simulant and stripping boric acid solutions of varying concentrations ${ }^{a, b, c}$

\begin{tabular}{|c|c|c|c|c|}
\hline \multirow[t]{2}{*}{ Stage } & \multicolumn{4}{|c|}{ Boric Acid Concentration } \\
\hline & $10 \mathrm{mM}$ & $10 \mathbf{m M}^{\mathbf{c}}$ & $20 \mathrm{mM}^{\mathrm{c}}$ & $50 \mathbf{m M}^{\mathrm{c}}$ \\
\hline Extraction & 44.4 & 45.1 & 44.3 & 47.0 \\
\hline Scrub 1 & 2.01 & 1.98 & 2.66 & 2.00 \\
\hline Scrub 2 & 0.82 & 0.84 & 0.86 & 0.84 \\
\hline Strip 1 & $1.03 \times 10^{-3}$ & $1.67 \times 10^{-2}$ & $1.02 \times 10^{-2}$ & $6.16 \times 10^{-3}$ \\
\hline Strip 2 & $6.98 \times 10^{-6}$ & $1.83 \times 10^{-3}$ & $4.37 \times 10^{-3}$ & $8.63 \times 10^{-4}$ \\
\hline Strip 3 & $6.48 \times 10^{-4}$ & $6.83 \times 10^{-4}$ & $1.08 \times 10^{-3}$ & $3.64 \times 10^{-4}$ \\
\hline
\end{tabular}

${ }^{a}$ The organic phase consisted of $50 \mathrm{mM}$ MaxCalix, $0.50 \mathrm{M} \mathrm{Cs-7SB}$ modifier, and denoted concentrations of LIX 79 guanidine (DCiTG) and Isopar L as the diluent. The aqueous phase consisted of the SRS-15 and SRS-45 waste simulant. The scrub solution was $25 \mathrm{mM} \mathrm{NaOH}$ and the strip solution consisted of $\mathrm{H}_{3} \mathrm{BO}_{3}$ 10-50 mM. O:A ratios were 1:4 for extraction and 3.75:1 for scrubbing and stripping. Each contact was made at $\sim 22-26^{\circ} \mathrm{C}$ and equilibrated for at least 60 minutes for extraction and 30 minutes for scrubbing and stripping.

${ }^{b}$ Values are background corrected.

${ }^{C}$ The SRS-15 waste simulant contained a higher concentration of cesium $(0.6 \mathrm{mM})$. 


\subsubsection{Further Testing of the NG-CSSX Solvent}

\subsubsection{Changing O:A ratios}

Changing the O:A ratios in the NG-CSSX process will potentially increase throughput in the contactors and could improve the hydraulic properties of the process, including lowering phase-separation times. The O:A values for ESS were changed from 1:3, 5:1 and 5:1 to 1:4, 3.75:1 and 3.75:1. In each stage the O:A ratio was reduced. Table 3.17 shows the results of ESS testing of the NG-CSSX solvent (solvent components shown in footnote of table) with the new O:A ratios, varying both the simulant composition and the concentration of the boric acid strip solution.

The $D_{C s}$ values are still maintained when the NG-CSSX solvent is subjected to ESS testing with both waste simulants (SRS-15 and SRS-45) at 2 stripping feed concentrations. This again confirms that, even with increased cesium transfer, the $10 \mathrm{mM}$ boric acid strip solution performs as well as the $100 \mathrm{mM}$.

Table 3.17. Cesium distribution ratios from ESS experiments with the SRS-15 and SRS-45 waste simulants using Reduced O:A Ratios ${ }^{a, b}$

\begin{tabular}{|c|c|c|c|c|}
\hline \multirow[t]{2}{*}{ Stage } & \multicolumn{2}{|c|}{ SRS-15 } & \multicolumn{2}{|c|}{ SRS-45 } \\
\hline & $10 \mathrm{mM} \mathrm{H}_{3} \mathrm{BO}_{3}$ & $100 \mathrm{mM} \mathrm{H}_{3} \mathrm{BO}_{3}$ & $10 \mathrm{mM} \mathrm{H}_{3} \mathrm{BO}_{3}$ & $100 \mathrm{mM} \mathrm{H}_{3} \mathrm{BO}_{3}$ \\
\hline Extraction & 55.4 & 60.3 & 51.3 & 51.0 \\
\hline Scrub 1 & 3.31 & 3.50 & 3.98 & 3.09 \\
\hline Scrub 2 & 1.75 & 1.76 & 2.02 & 1.98 \\
\hline Strip 1 & $2.19 \times 10^{-3}$ & $6.13 \times 10^{-4}$ & $2.81 \times 10^{-3}$ & $6.14 \times 10^{-4}$ \\
\hline Strip 2 & $5.64 \times 10^{-4}$ & $1.97 \times 10^{-4}$ & $6.22 \times 10^{-4}$ & $2.62 \times 10^{-4}$ \\
\hline Strip 3 & $4.49 \times 10^{-4}$ & $3.68 \times 10^{-4}$ & $7.22 \times 10^{-4}$ & $3.12 \times 10^{-4}$ \\
\hline
\end{tabular}

${ }^{\mathrm{a}}$ The organic phase consisted of $50 \mathrm{mM}$ MaxCalix, $0.50 \mathrm{M}$ Cs-7SB modifier, and 0.003 M LIX 79 guanidine (DCiTG) and Isopar L as the diluent. The aqueous phase consisted of the SRS-15 and SRS-45 waste simulant. The scrub solution was $25 \mathrm{mM} \mathrm{NaOH}$ and the strip solution consisted of 10 or $100 \mathrm{mM} \mathrm{H}_{3} \mathrm{BO}_{3}$. New O:A ratios were 1:4 for extraction and 3.75:1 for scrubbing and stripping. Each contact was made at $\sim 22-26^{\circ} \mathrm{C}$ and equilibrated for at least 60 minutes for extraction and 30 minutes for scrubbing and stripping.

${ }^{b}$ Values are background corrected.

\subsubsection{Solvent robustness after multiple $\mathbf{E S}_{2} \mathbf{S}_{3}$ cycles}

The robustness of the initial solvent (20 mM MaxCalix, 0.5 M Cs-7SB and $3 \mathrm{mM}$ DCiTG) was tested by conducting a multi-cycle $\mathrm{ES}_{2} \mathrm{~S}_{3}$ experiment using SRS-15, $25 \mathrm{mM} \mathrm{NaOH}$ and $100 \mathrm{mM} \mathrm{H}_{3} \mathrm{BO}_{3}$ as the aqueous phases (O:A ratios were 1:3 for extraction and 5:1 for scrubbing and stripping). The solvent was cycled through seven complete $\mathrm{ES}_{2} \mathrm{~S}_{3}$ tests and was compared with a second batch of solvent that went through a $\mathrm{NaOH}(10 \mathrm{mM})$ washing stage between cycles, Table 3.18. The extraction $D_{C s}$ values appears to rise when comparing the first cycles, but then decreases in the final seventh stage. This same trend can 
be observed in both the scrubbing and stripping stages, with the $D_{C s}$ values increasing sligtly then returning to normal $D_{C s}$ in the final cycle of the experiment.

The ten-cycle ESS with wash experiment was carried out to see if a wash stage would result in any noticeable changes in the $D_{C s}$ values after multiple cycles. $0.010 \mathrm{M} \mathrm{NaOH}$ was selected as the washing solution since it is used to wash the current BOBCalixC6 CSSX solvent in the MCU at SRS. After ten full cycles $\left(\mathrm{ES}_{2} \mathrm{~S}_{3} \mathrm{~W}_{2}\right)$, the solvent appears to function as it had in the earlier tests. The $D_{C s}$ values shown below in Table 3.19 show little deviation from the previously reported $D_{C s}$ value for an ESS cycle. The $D_{C s}$ values for the first and tenth cycle are almost identical, showing the solvent to perform as designed for at least ten cycles on a benchtop scale. A slight increase in extraction and scrubbing $D_{C s}$ was seen during the fifth cycle with the solvent returning to the expected values by the tenth cycle. The cause of the increase in the $D_{C s}$ in the fifth cycle is unknown, although it is postulated that there may have been a small amount of impurities in the solvent. This recovery of $D_{C S}$ values suggests that the process is fairly robust when it comes to the removal of possible impurities in the solvent (for more information on the effect of impurities in the NG-CSSX process see Chapter 11). 


\subsection{CONCLUSIONS}

The preferred calixarene is the more soluble extractant MaxCalix, due to its excellent resistance to thirdphase formation. The use of a guanidine derivative (DCiTG active component of LIX 79) provides very efficient stripping, regardless of the concentration of boric acid used in the stripping stages. The increase in calixarene concentration made possible by the improved solubility of MaxCalix allows a decrease of the modifier Cs-7SB concentration from $0.75 \mathrm{M}$ to $0.5 \mathrm{M}$, which is expected to improve hydraulics in the industrial process.

The introduction of a sodium hydroxide $(0.025 \mathrm{M})$ scrub enabled successful removal of potassium from the solvent whilst maintaining $D_{C s}$ values $>1$. The boric acid stripping provides the ideal combination of acidity and hydrophilic anion to efficiently strip the cesium from the solvent. A concentration of $0.01 \mathrm{M}$ is high enough for successful stripping but not top high to cause potential third phase issues.

Actual flowsheet performance is expected to depend strongly on contactor stage efficiency, given that very high and very low $D_{\mathrm{Cs}}$ values are respectively obtained in extraction and stripping. Based on stage efficiency estimations of current MCU performance in the range of $80-85 \%$, it has been considered that the higher MaxCalix concentration of $50 \mathrm{mM}$ in the solvent, other components remaining unchanged, would provide a greater margin or robustness in reaching the target DF of $>40,000$, while still being able to reduce O:A on extraction for a throughput gain ${ }^{3}$. In summary, NG-CSSX solvent chemistry has been advanced to provide a flowsheet performance expected to meet different needs in the cleanup of US DOE wastes.

The composition of the NG-CSSX solvent used throughout the rest of this report is as follows; Extractant: MaxCalix (0.05 M), Modifier: Cs-7SB (0.50 M), Suppressor: DCiTG (0.003 M) in Isopar L. The determined aqueous feeds are sodium hydroixe $(0.025 \mathrm{M})$ for scrubbing and boric acid $(0.01 \mathrm{M})$ for stripping.

The following chapter details the synthesis and characterization of the DCiTG suppressor, required to yield the high purity required for successful full-scale industrial operations. 



\section{GUANIDINE PREPARATION}

Procedures for the synthesis of isotridecylamine (iTDA), DCiTG, and 1,3-dicyclohexyl-2(isotridecyl)guanidine hydrochloride $(\mathrm{DCiTG} \cdot \mathrm{HCl}$ ) are described. DCiTG (a generic version of the active guanidine reagent in the Cognis extractant LIX $^{\circledR} 79$ ) is a key component of the ORNL NG-CSSX process solvent.

\subsection{INTRODUCTION}

This chapter provides detailed synthetic procedures for preparing DCiTG, and DCiTG $\bullet \mathrm{HCl}$. The format employed by Organic Syntheses, Inc. (www.orgsyn.org/) for describing detailed synthetic procedures for making fine organic chemicals has been adopted, since "Organic Syntheses" procedures are well known for their attention to detail, identification of problem areas and safety issues, and overall clarity. Background information on the need for synthetic procedures for DCiTG and DCiTG $\bullet \mathrm{HCl}$ can be found below in the Discussion Section 4.3.

\subsection{EXPERIMENTAL SECTION}

\subsubsection{Preparation of 2-(isotridecyl)isoindoline-1,3-dione (1)}

A modification of the procedure described ${ }^{15}$ was employed. To a $2 \mathrm{~L}$ round-bottom flask was added, under nitrogen, triphenylphosphine $(131.15 \mathrm{~g}, 0.50 \mathrm{~mol})$, phthalimide $(73.57 \mathrm{~g}, 0.50 \mathrm{~mol})$, EXXAL 13 tridecyl alcohol (100.2 g, nominally $0.50 \mathrm{~mol}$ ) (Note 1), $600 \mathrm{~mL}$ dry ethyl ether, and a stir bar. The flask was fitted with a 250-mL pressure-equalizing addition funnel (Note 2) containing 94\% diisopropylazodicarboxylate (DIAD, $107.6 \mathrm{~g}$, nominally $0.50 \mathrm{~mol}$ ) (Note 3) dissolved in $100 \mathrm{~mL}$ of dry ether, fitted with a nitrogen inlet to bubbler outlet at the top. The rapidly stirred contents of the flask were cooled in an ice-bath to $\sim 0-1{ }^{\circ} \mathrm{C}$ under nitrogen (Note 4). The ether solution of DIAD was added dropwise over 75 min, during which time the bath temperature was maintained below $10^{\circ} \mathrm{C}$ by the periodic addition of ice. About halfway through the addition the reaction mixture became translucent and yellow, after which more solids began to form. The cold bath was removed, and the reaction was allowed to stir overnight $(21 \mathrm{~h})$ at ambient temperature under nitrogen during which time off-white solids formed in the yellow solution in the reaction flask. The slurry was then filtered and the solids washed with ether until the solid was white and the filtrate was colorless. The mass of the recovered solids (triphenylphosphine oxide and diisopropyl hydrazodicarboxylate, in a nearly equimolar ratio by proton Nuclear Magnetic Resonance [NMR]) was $195 \mathrm{~g}$ ( $~ 81 \%$ of theory), indicating that about $46 \mathrm{~g}$ of these side products would be present in the product. The ether was removed from the collected filtrate by rotary evaporation $(1 \mathrm{~L}$ 
flask) to give $219 \mathrm{~g}$ of the crude product as a yellow oil. Though the crude product (obtained at quantitative yield) contains some ether, as well as the balance of the triphenylphosphine oxide and diisopropyl hydrazodicarboxylate (by NMR analysis), the material can be used in the next step without purification (Notes 5 and 6).

\subsubsection{Preparation of iTDA (2)}

To the $1 \mathrm{~L}$ round-bottom flask containing nominally $0.5 \mathrm{~mol}$ of the crude 2-(isotridecyl)isoindoline-1,3dione, 1, above, was added $250 \mathrm{~mL}$ of absolute ethanol and a stir bar. After dissolution of the dione was complete, hydrazine hydrate (35.0 g, 0.70 mol, Caution! Note 7) was added. An Allihn-type condenser with nitrogen inlet was then attached, and the solution immersed in a pre-heated $85^{\circ} \mathrm{C}$ oil bath (Note 8). The reaction was heated overnight $(18 \mathrm{~h})$ under nitrogen, during which time a pale yellow solid mass formed. A solution of $100 \mathrm{~mL} 12 \mathrm{~N} \mathrm{HCl}$ in $125 \mathrm{~mL}$ absolute ethanol was carefully added (Note 9), and heating continued at $85{ }^{\circ} \mathrm{C}$ for an additional 30 min. Heating was then terminated, and the flask was allowed to cool in the air, and then cooled in an ice bath. The solid mass of 2,3-dihydrophthalazine-1,4dione (the byproduct of the deprotection) was broken up and the slurry was filtered. The off-white 2,3dihydrophthalazine-1,4-dione was washed first with four $50-\mathrm{mL}$ portions of distilled deionized water, followed by one $50-\mathrm{mL}$ portion of $95 \%$ ethanol (Note 10 ). 


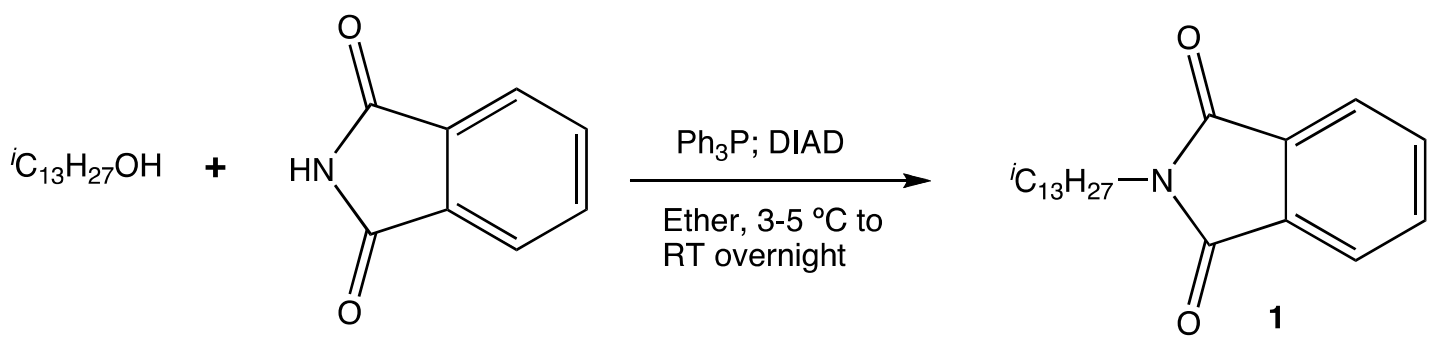

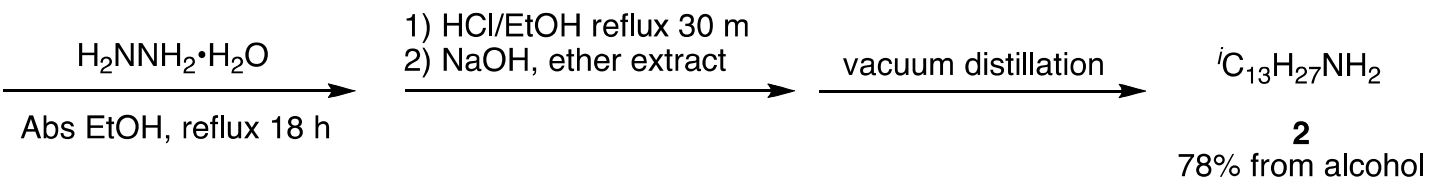

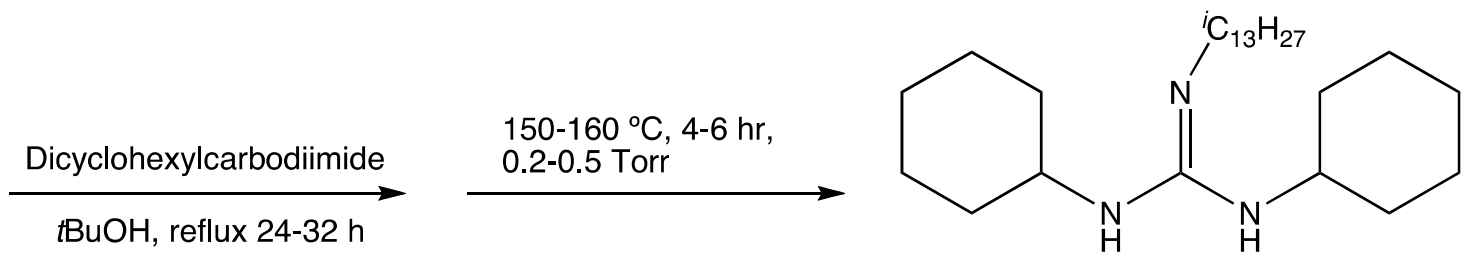

3, DCITG

quantitative from amine

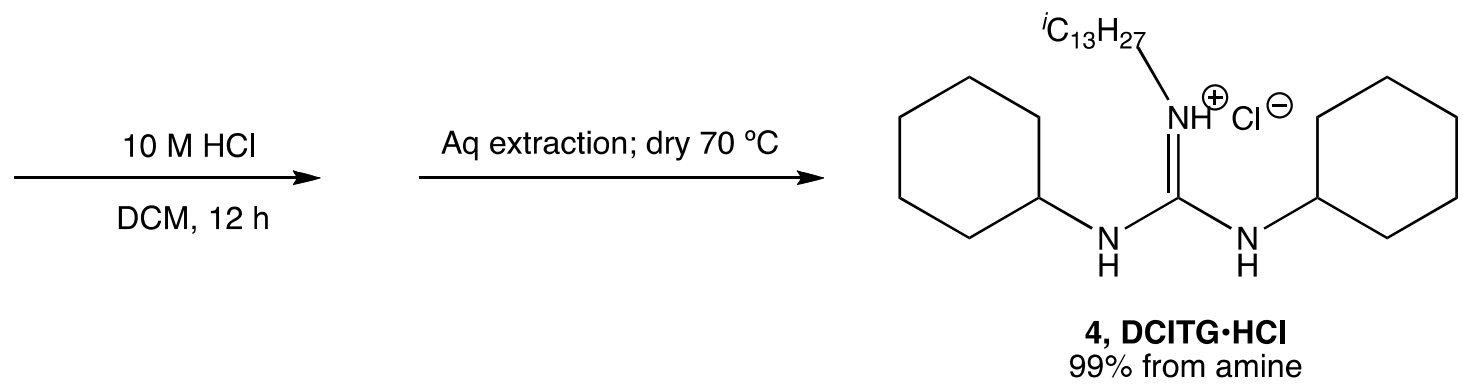

Fig. 4.1. Synthetic procedure for 1,3-Dicyclohexyl-2-(isotridecyl)guanidine hydrochloride.

The combined pale yellow filtrate is transferred to a $1 \mathrm{~L}$ evaporating flask, and the volume reduced by rotary evaporation to yield $420 \mathrm{~g}$ of a turbid yellow oil. To release the amine, $3.5 \mathrm{~N} \mathrm{NaOH}$ was added at room temperature to the oil, until $\mathrm{pH} 14$ was reached $(200 \mathrm{~mL})$. The biphasic mixture (Note 11) was then extracted with diethyl ether $(3 \times 500 \mathrm{~mL})$ (Note 12$)$, and the ether solution dried over anhydrous sodium sulfate. The ether was removed by rotary evaporation $\left(40{ }^{\circ} \mathrm{C}\right)$, followed by evaporation at $50{ }^{\circ} \mathrm{C}$ and 15 torr (Note 13) to give $140.45 \mathrm{~g}$ of the crude amine as a brown oil. The oil was allowed to stand at room temperature for two weeks (Note 14) during which time most of the residual triphenylphosphine oxide and diisopropyl hydrazodicarboxylate crystallized out. The light brown supernatant liquid phase ( $80 \mathrm{~g})$ was transferred to a $250-\mathrm{mL}$ distilling flask. The light brown solids that remained were washed with $5 \times$ 
$15 \mathrm{~mL}$ pentane (Note 15), and the washings combined in the distilling flask with the bulk brown oil. Most of the pentane was then removed by rotary evaporation to give $100 \mathrm{~g}$ of crude amine (Note 16). The crude amine was distilled under vacuum (short path), collecting the colorless liquid fraction that distills between $60{ }^{\circ} \mathrm{C}$ at $0.080 \mathrm{mmHg}$ (about $273{ }^{\circ} \mathrm{C}$ at $760 \mathrm{mmHg}$ ) and $67{ }^{\circ} \mathrm{C}$ at $0.055 \mathrm{mmHg}$ (about $291{ }^{\circ} \mathrm{C}$ at 760 $\mathrm{mmHg}$ ) (Note 17). The yield of the distilled iTDA so obtained was $77.95 \mathrm{~g}$ (78 \% yield from EXXAL 13 based on a nominal formula weight of $199.38 \mathrm{~g} /$ mole for $\mathrm{C}_{13} \mathrm{H}_{29} \mathrm{~N}$ iTDA) with a purity of $98+\%$ (Note 18).

\subsubsection{Preparation of 1,3-Dicyclohexyl-2-(isotridecyl)guanidine (3)}

A modification of the procedure described ${ }^{16}$ was employed. $N, N^{\prime}$-dicyclohexylcarbodiimide (DCC, $6.06 \mathrm{~g}, 29.4 \mathrm{mmol}, 1 \mathrm{eq}$ ) was added to a $100-\mathrm{mL}$ round-bottom flask containing a magnetic stir bar. The flask was evacuated and refilled with argon using 3 vacuum/refill cycles. Anhydrous tert-butyl alcohol $(40 \mathrm{~mL})$ (Note 19) was added to the flask, and a condenser was attached. The entire apparatus was again evacuated and refilled with argon using 3 vacuum/refill cycles. After complete dissolution of the DCC, iTDA (7.34 g, $36.8 \mathrm{mmol}, 1.25 \mathrm{eq}$ ) was added to the solution (Note 20) and refluxed at $90{ }^{\circ} \mathrm{C}$ overnight $(18 \mathrm{~h})$ under argon atmosphere, during which the initially clear solution turned pale yellow. The reaction was monitored via gas chromatography (GC) and upon completion of the reaction (24-32h), the solvent was removed on a rotary evaporator to obtain a viscous pale yellow colored oil. The oil was then stripped of the residual amine by Kugelrhor (bulb-to-bulb) distillation at $150-160^{\circ} \mathrm{C}$ at $0.2-0.5$ Torr for $4-6$ hours to give $11.91 \mathrm{~g}$ of pale yellow viscous oil. Analysis by GC (Note 21) for the presence of residual amine indicated a purity for DCiTG of $\geq 99 \%$, and hence a yield of $\geq 99 \%$ (Note 22.).

\subsubsection{Preparation of DCiTG•HC1 (4)}

The purified DCiTG, $\mathbf{3}$, is dissolved in $100 \mathrm{~mL}$ methylene chloride and stirred with $100 \mathrm{~mL}$ of $10 \mathrm{M} \mathrm{HCl}$ for 12 hours. The two-phase system is transferred to a separatory funnel, where the acid layer is removed, and the organic layer washed 3 times with $100 \mathrm{~mL}$ deionized water. The solvent is then removed by rotary evaporation (Note 23), and the residue dried overnight in a vacuum oven at $70^{\circ} \mathrm{C}$ to give 12.75 grams $(99 \%)$ of DCiTG $\bullet \mathrm{HC} 1, \mathbf{4}$, as a pale yellow/amber glass (Note 24 .)

\subsubsection{Notes}

1. All reagents were used as received from the suppliers without further purification unless otherwise noted. A one liter sample of EXXAL 13 "tridecyl" alcohol ( 88\% C11-C13 branched alcohols, 
balance C9, 10, 14 alcohols, Lot AANB3 with test date 8/25/95) was received as a gift from ExxonMobil.

2. Teflon ${ }^{\circledR}$ joint sleeves (VWR brand) were used at all ground glass joints.

3. Diisopropylazodicarboxylate (AlfaAesar, 94\%) is preferred over diethylazodicarboxylate (DEAD) since it is somewhat cheaper, and the byproduct diisopropyl hydrazodicarboxylate (CAS 19740-72-8) has a higher boiling point than the analogous diethyl hydrazodicarboxylate (CAS 4144-28-7). The bp of diisopropylhydrazodicarboxylate is slightly higher than the highest boiling fractions of the iTDA mixture from EXXAL 13. The amount of DIAD added was calculated based on 94\% purity.

4. Not everything is soluble in the beginning but that does not appear to hinder the reaction.

5. For convenience, a $1 \mathrm{~L}$ flask was selected for ether removal, as the subsequent reaction can be performed on the obtained crude dione material directly in this $1 \mathrm{~L}$ flask.

6. Proton, carbon, and 2D-COSY NMR spectra were obtained on a Varian VNMRS 500 NMR spectrometer, operating at $499.715 \mathrm{MHz}$ for proton. All spectra were recorded at room temperature in $\mathrm{CDCl}_{3}$ and referenced to internal tetramethylsilane $(0 \mathrm{ppm})$ for proton spectra, and to $77.23 \mathrm{ppm}$ for $\mathrm{CDCl}_{3}$ for carbon spectra, unless otherwise noted. ${ }^{1} \mathrm{H}$ NMR for product: $\delta 7.84(\mathrm{~m}, 2 \mathrm{H}$, aromatic), $7.70\left(\mathrm{~m}, 2 \mathrm{H}\right.$, aromatic), $3.68\left(\mathrm{~m}, 2 \mathrm{H},-\mathrm{CH}_{2} \mathrm{CH}_{2} \mathrm{~N}\right), 1.67$ (br m, 2H, $\left.-\mathrm{CH}_{2} \mathrm{CH}_{2} \mathrm{~N}\right), 1.5-1.4$ (br overlapping, $\sim 1 \mathrm{H}, \quad-\mathrm{CH}$, assignment based on COSY spectrum), 1.4-1.0 (br, $\sim 16 \mathrm{H}, \quad-$ $\mathrm{CH}\left(\mathrm{CH}_{2}\right)_{8(\text { ave) }} \mathrm{CH}_{2} \mathrm{CH}_{2} \mathrm{~N}$ ), 0.9-0.7 (br m, $\sim 6 \mathrm{H},-\mathrm{CH}_{3}$, Note: there is considerable overlap with the methyl groups from residual diisopropylhydrazodicarboxylate. ${ }^{13} \mathrm{C}\left\{{ }^{1} \mathrm{H}\right\}$ NMR $\left(\mathrm{CDCl}_{3}\right.$; due to the many different alkyl chain lengths, the ${ }^{13} \mathrm{C}$ spectrum is complex, particularly in the $40-10 \mathrm{ppm}$ region): $\delta 168.6,168.5$ (br, C=O's), 133.8, 132.3, 123.2 (aromatic; these are doubled peaks with 3-5 $\mathrm{Hz}$ separation), 38.5, 38.2, $36.5\left(-\mathrm{CH}_{2} \mathrm{CH}_{2} \mathrm{~N}\right.$ from various alkyl chains, based on corresponding pattern of 63.6, 63.2, and $61.4 \mathrm{ppm}$ from starting alcohol), 40-10 (many peaks due to alkyl chains and methyl groups).

7. Caution: hydrazine hydrate is a highly toxic material and a suspected carcinogen, and should only be handled in a functioning fume hood with appropriate personal protective equipment. In this example 1.4 molar equivalents (on the dione) of hydrazine hydrate was used, but the lesser amounts (e.g., as low as 1.1 molar equivalents) can be used, but for best results an excess should be used.

8. A clear yellow solution initially forms when the flask is immersed in the preheated oil bath, but a thick pale-yellow cake forms within 5 minutes. It may be necessary to periodically break up the cake.

9. The acid, usually at least 2.2 molar equivalents on the dione, is added to the solution while hot, and can be added down the condenser into the reaction flask.

10. The aqueous washes alone may not be sufficient to wash out any entrained amine hydrochloride, given the lipophilicity of the alkyl chains, hence the need for the additional $95 \%$ ethanol wash 
(though excessive ethanol should be avoided to prevent the resolubilization of the 2,3dihydrophthalazine-1,4-dione.) The filtrate of the last aqueous wash should be colorless or nearly colorless. In subsequent syntheses, we are now washing first with ethanol, followed by the aqueous washings.

11. The organic upper layer is typically olive to light brown in color.

12. The color of the first extraction is typically olive to brown, the color of the second extraction is typically light yellow-peach, and the color of the third extraction is typically very pale yellow/peach to nearly colorless.

13. The higher temperature and vacuum allows residual ethanol to be removed.

14. The color of the crude amine can range from yellow to brown. Allowing the crude mixture to sit for two days is usually sufficient for most of the triphenylphosphine oxide and diisopropyl hydrazodicarboxylate to precipitate.

15. Enough pentane should be used so that the solids (residual triphenylphosphine oxide and diisopropyl hydrazodicarboxylate), which are mostly insoluble in pentane, are rendered nearly white to ensure adequate removal of adsorbed crude amine. The mass of washed solids was about $45 \mathrm{~g}$, affording near complete mass balance when combined with the $195 \mathrm{~g}$ initial precipitate obtained in part A.

16. We found it more convenient to remove the pentane on the rotary evaporator prior to vacuum distillation.

17. In this example, the smaller chain, lighter boiling fraction distilled between $60^{\circ} \mathrm{C}$ at $0.080 \mathrm{mmHg}$ and $62{ }^{\circ} \mathrm{C}$ at $0.070 \mathrm{mmHg}\left(273-279{ }^{\circ} \mathrm{C}\right.$ at $\left.760 \mathrm{mmHg}\right)$. The major fraction, constituting perhaps $70-80 \%$ of the bulk, distilled between $62^{\circ} \mathrm{C}$ at $0.070 \mathrm{mmHg}$ and $65^{\circ} \mathrm{C}$ at $0.060 \mathrm{mmHg}\left(279-286{ }^{\circ} \mathrm{C}\right.$ at 760 $\mathrm{mmHg}$ ). The remaining longer chain fraction distilled between $65^{\circ} \mathrm{C}$ at $0.00 \mathrm{mmHg}$ and $67{ }^{\circ} \mathrm{C}$ at $0.055 \mathrm{mmHg}\left(286-291{ }^{\circ} \mathrm{C}\right.$ at $\left.760 \mathrm{mmHg}\right)$. The oil bath temperature was gradually raised from $80{ }^{\circ} \mathrm{C}$ to $95{ }^{\circ} \mathrm{C}$ during the distillation of the combined fractions. No forerun was collected separately, as the volatile components (residual pentane and ethanol mostly) report to the trap.

18. Any residual diisopropyl hydrazodicarboxylate co-distills with the higher boiling fraction. We observed about 2\% residual diisopropyl hydrazodicarboxylate in the product. Higher purity amine, with a slightly narrower molecular weight distribution, can be obtained by fractionating the distillate (e.g., collecting the fraction which distills above about $286-288{ }^{\circ} \mathrm{C}$ at $760 \mathrm{mmHg}$ separately.) ${ }^{1} \mathrm{H}$ NMR: $\delta 2.67$ (br m, 2H, $-\mathrm{CH}_{2} \mathrm{~N}$ ), 1.65-0.95 (three br overlapping peaks, $\sim 15-16 \mathrm{H}$, alkyl chain); 0.950.70 (overlapping peaks, $\sim 12-13 \mathrm{H}$, all methyl groups). The pattern for the alkyl chain portion is very similar to that of the starting EXXAL 13, and there seem to be on average at least 4 methyl groups. ${ }^{13} \mathrm{C}\left\{{ }^{1} \mathrm{H}\right\}$ NMR $\left(\mathrm{CDCl}_{3}\right)$; due to the many different alkyl chain lengths, the ${ }^{13} \mathrm{C}$ spectrum is complex): 
$\delta 42.9,42.5,40.3\left(-\mathrm{CH}_{2} \mathrm{~N}\right.$ from various alkyl chains, based on corresponding pattern of $63.6,63.2$, and 61.4 ppm from starting alcohol), 44.5-8.6 (many peaks due to alkyl chains and methyl groups).

19. Anhydrous tert-butanol must be used to minimize the formation of dicyclohexylurea.

20. An excess of iTDA was added to ensure complete consumption of the DCC during the reaction. During the distillation, the amine is easy to remove, but DCC and the other impurities and byproducts cannot be stripped without thermal degradation of the guanidine.

21. The compound was analyzed using an Agilent/HP 6850 GC equipped with a FID detector. The inlet was set to split injection at a 50:1 ratio (split flow $108.2 \mathrm{~mL} / \mathrm{min}$, total flow $115.2 \mathrm{~mL} / \mathrm{min}$ ). The injection port was set at a temperature of $280{ }^{\circ} \mathrm{C}$. The column used was an HP-5MS $(5 \%$ Phenyl methyl siloxane) open capillary column with a $30 \mathrm{~m}$ length, $0.25 \mathrm{~cm}$ inner diameter, and stationaryphase thickness of $0.25 \mu \mathrm{m}$. The $\mathrm{GC}$ used $\mathrm{H}_{2}$ as the carrier gas, set in constant flow mode at 2.2 $\mathrm{mL} / \mathrm{min}$ (a nominal head pressure of $14.30 \mathrm{psig}$, to give an average linear velocity of $60 \mathrm{~cm} / \mathrm{sec}$ ). The detector was set at $280^{\circ} \mathrm{C}$, with an $\mathrm{H}_{2}$ flow of $50 \mathrm{~mL} / \mathrm{min}$, an air flow of $450 \mathrm{~mL} / \mathrm{min}$, and no makeup gas. Using this set up, the product, reactants, and crude reaction mixtures were analyzed using the temperature program: $100{ }^{\circ} \mathrm{C}$ ramp of $20^{\circ} \mathrm{C} / \mathrm{min}$ to a final temperature of $300{ }^{\circ} \mathrm{C}$, then held at $300{ }^{\circ} \mathrm{C}$ for $3 \mathrm{~min}$. The integrator was started at $2.0 \mathrm{~min}$ and recorded signal to the end of the run. See Chapters 5 and 6 for further details. See discussion for sample chromatogram and purity analysis by GC and GC/MS.

22. Proton and carbon NMR spectra for this compound were obtained on a Bruker 400 NMR spectrometer, operating at $400.13 \mathrm{MHz}$ for proton. All spectra were recorded at room temperature in $\mathrm{CDCl}_{3}$ and referenced to internal tetramethylsilane $(0 \mathrm{ppm})$ for proton spectra, and to $77.23 \mathrm{ppm}$ for $\mathrm{CDCl}_{3}$ for carbon spectra, unless otherwise noted. ${ }^{1} \mathrm{H}$ NMR for product: $\delta 3.16$ (br, $2 \mathrm{H}$, cyclohexyl$\mathrm{CH}), 2.99$ (d, J=8.0 Hz, 2H, NH), 1.87 (d, J=12.0 Hz), 1.71 (d, J=8.0 Hz), 1.57 (d, J=12.0 Hz), 1.261.17 (br m), 0.85-0.73 (br m). (The peaks in the region 0.73-1.87 are overlapping with each other and accounts for 47 protons with respect to two NH protons at 2.99 and two cyclohexyl-CH protons at $3.16 \mathrm{ppm}$. In addition, the NH peaks are found to shift downfield, dependent on the degree of protonation of the guanidine, 2.99-7.30). ${ }^{13} \mathrm{C}\left\{{ }^{1} \mathrm{H}\right\}$ NMR (due to the many different alkyl chain lengths, the ${ }^{13} \mathrm{C}$ spectrum is complex, particularly in the $45-10 \mathrm{ppm}$ region): $\delta 152.2(\mathrm{C}=\mathrm{O}), 51.6$ (cyclohexyl-CH-), 34.2, 26.8, 45-10 (many peaks due to alkyl chains and methyl groups).

23. The DCiTG holds on the solvent and begins to foam as the last traces of solvent are removed. By removing the solvent using a bath temperature of $70{ }^{\circ} \mathrm{C}$ this is minimized because the salt melts at approximately $60^{\circ} \mathrm{C}$. Once the bulk of the solvent is removed, it is recommended to incrementally decrease the rotovap pressure to 20 Torr, and once stable at 20 Torr (no more foaming), continue 
drying the material in a drying oven. A salt that is not excessively dried prior to going in the drying oven will produce a lot of foam and possibly create a mess.

24. ${ }^{1} \mathrm{H}$ NMR (400 MHz, $\mathrm{CDCl}_{3}$ ) $\delta 7.69$ (br s, 1H), 6.97 (br s, 2H), 3.58 (br s, 2H), 3.24 (br s, 2H), 1.91 $(\mathrm{d}, J=9.6 \mathrm{~Hz}, 4 \mathrm{H}), 1.74(\mathrm{~d}, J=13.0 \mathrm{~Hz}, 4 \mathrm{H}), 1.68-0.98(\mathrm{~m}, 26 \mathrm{H}), 0.94-0.65(\mathrm{~m}, 11 \mathrm{H}) .{ }^{13} \mathrm{C} \mathrm{NMR}$ $\left(101 \mathrm{MHz} \mathrm{CDCl}_{3}\right) \delta 155.87,52.63,33.08,25.04,24.73$, plus a complex mixture of small peaks between $45-10 \mathrm{ppm}$.

\subsection{DISCUSSION}

\subsubsection{Synthesis}

\subsubsection{1,3-dicyclohexyl-2-(isotridecyI)guanidine}

DCiTG (a generic version of the active guanidine reagent in the Cognis extractant LIX 79) is a key component of the ORNL NG-CSSX process solvent ${ }^{11}$. An evaluation of the performance of several 1,3dicyclohexyl-2-(alkyl)guanidines in the NG-CSSX process showed that the DCiTG component of LIX 79 appeared to be better than other guanidines, such as 1,3-dicyclohexyl-2-(3,7-dimethyloctyl)guanidine and 1,3-dicyclohexyl-2-(2-ethylhexyl)guanidine. However, the DCiTG component of LIX 79 guanidine is not available in the quantities needed for the NG-CSSX solvent; hence there is a need for a procedure to specifically prepare this material. The guanidine as the free base is physically a viscous oil, hence the hydrochloride form (DCiTG $\cdot \mathrm{HCl}$ ), which is a glassy solid that can be converted to a white powder, may be easier to handle when preparing the NG-CSSX solvent.

The guanidine can be prepared by the reaction of dicylohexylcarbodiimide with iTDA ${ }^{16}$, wherein the iTDA (CAS No. 35723-81-0) is not a single material but consists of a complex mixture of primarily isomeric C13 amines. The commercial availability of this amine appears to be limited. However the amine can be prepared from industrial isotridecanol, a complex mixture of branched C10-C14 primary alcohols wherein the greatest proportion is the $\mathrm{C} 13$ chain length. The alcohol is readily available, and commercial sources of isotridecanol include ExxonMobil (EXXAL 13, (CAS No. 68526-86-3) and BASF (Isotridecanol N, CAS No. 27458-92-0).

One method for converting a primary alkyl alcohol to the corresponding primary alkyl amine involves the two-step procedure of first preparing the 2-(alkyl)isoindoline-1,3-dione by reacting the alcohol with phthalimide under Mitsunobu-type conditions, ${ }^{17}$ followed by removal/deprotection of the phthalimide group using hydrazine hydrate. This procedure has proved to be fairly efficient, affording fairly high yields on the individual steps, such that yields on the amine from the alcohol of $>70 \%$ are achievable. 
The first step to generate the 2-(alkyl)isoindoline-1,3-dione results in the production of triphenylphosphine oxide and dialkyl hydrazodicarboxylate (from the dialkyl azodicarboxylate used) as byproducts. These byproducts mostly precipitate out during the reaction, and can be filtered off, though it is important to wash the precipitates with ether to remove adsorbed dione. Accordingly about $20 \%$ of these byproducts carryover into the crude dione product. We have found it is easier to carry these byproducts through the next step rather than attempt to further purify the crude dione, since the product amine can be purified by distillation. However, though diethyl azodicarboxylate (DEAD) is the more commonly used reagent in the Mitsunobu reaction, we selected diisopropyl azodicarboxylate since it is somewhat cheaper and makes the higher boiling point byproduct diisopropyl hydrazodicarboxylate. The diisopropyl hydrazodicarboxylate distills just after the highest boiling point fraction of the iTDA mixture, as opposed to possibly co-distilling with the lower boiling fractions, as is probably the case for diethyl hydrazodicarboxylate. The measured boiling points for these have not been reported in the literature.

The crude dione can be reacted directly with excess hydrazine hydrate in absolute ethanol to, after workup, liberate the free amine. We have found it best to allow the crude amine to stand for several days to allow the remaining triphenylphosphine oxide and diisopropyl hydrazodicarboxylate to precipitate. The supernatant crude amine can then be transferred to a distilling flask. The triphenylphosphine oxide and diisopropyl hydrazodicarboxylate are insoluble in light hydrocarbons, and the precipitate can be washed with several portions of pentane (or petroleum ether) to remove adsorbed amine. The washings are combined with the crude amine, the pentane removed by rotary evaporation, and the amine distilled via short path vacuum distillation. The boiling point range for the mixture of amines produced from the parent EXXAL 13 isotridecanol appears to be about $273-291^{\circ} \mathrm{C}$ at $760 \mathrm{mmHg}$, based on conversion from the observed boiling points (see Note 17). The material distilling in this range constituted a yield of $78 \%$ amine based on the starting EXXAL 13.

The final step of the reaction involves the reaction between DCC and iTDA to obtain the required guanidine. The starting material DCC is a known dehydrating agent in preparation of amides, ketones, and nitriles, and in the presence of moisture gets converted to $N, N^{\prime}$-dicyclohexylurea (DCU) over time. Hence, it is better to start with fresh DCC for the reaction. An excess of amine (10-25\%) helps in complete consumption of the DCC and limits the formation of DCU. The choice of solvent was made following trials with dry tetrahydrofuran and dry p-dioxane, with the best progress obtained with tertbutanol $^{16}$. 


\subsubsection{GC analysis of DCiTG}

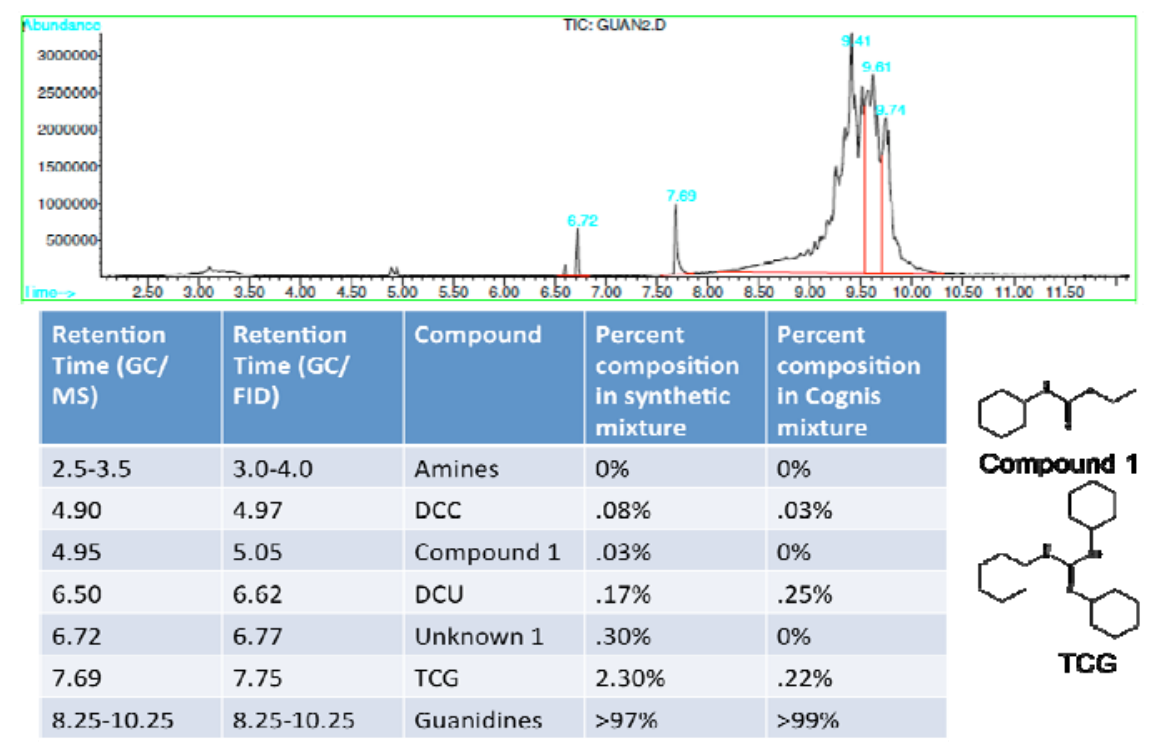

Fig. 4.2. GC chromatogram of crude DCiTG with retention time and compound assignments

The largest impurity in the synthetic DCiTG is tricyclohexylguanidine. Because the properties of tricyclohexylguanidine are so similar to DCiTG, it cannot be removed from the product mixture. It is doubtful that its presence will interfere with the function of DCiTG because it is another base with a very similar pKa to DCiTG. Unknown 1 is a carryover from the DCC used. It is present in the batch of DCC purchased at $97 \%$ purity from Oakwood, but 99\% pure DCC from Fluka does not contain it. The impurity is likely an organic phosphine oxide catalyst used in the condensation of cyclohexylisocyanate to DCC and its low concentration is of little concern. 


\subsubsection{Thermal stability of DCiTG and its salts}

DCiTG is a strong organic base with a pKa around 13.0-13.6 ${ }^{11}$. As such, any DCiTG that has contacted water can be expected to be fully protonated. Contact with water yields the hydroxide salt while contact with $\mathrm{HCl}$, the hydrochloride salt. Thermogravimetric analysis was used to determine the thermal decomposition temperature for the free base, the hydroxide salt and the hydrochloride salt.

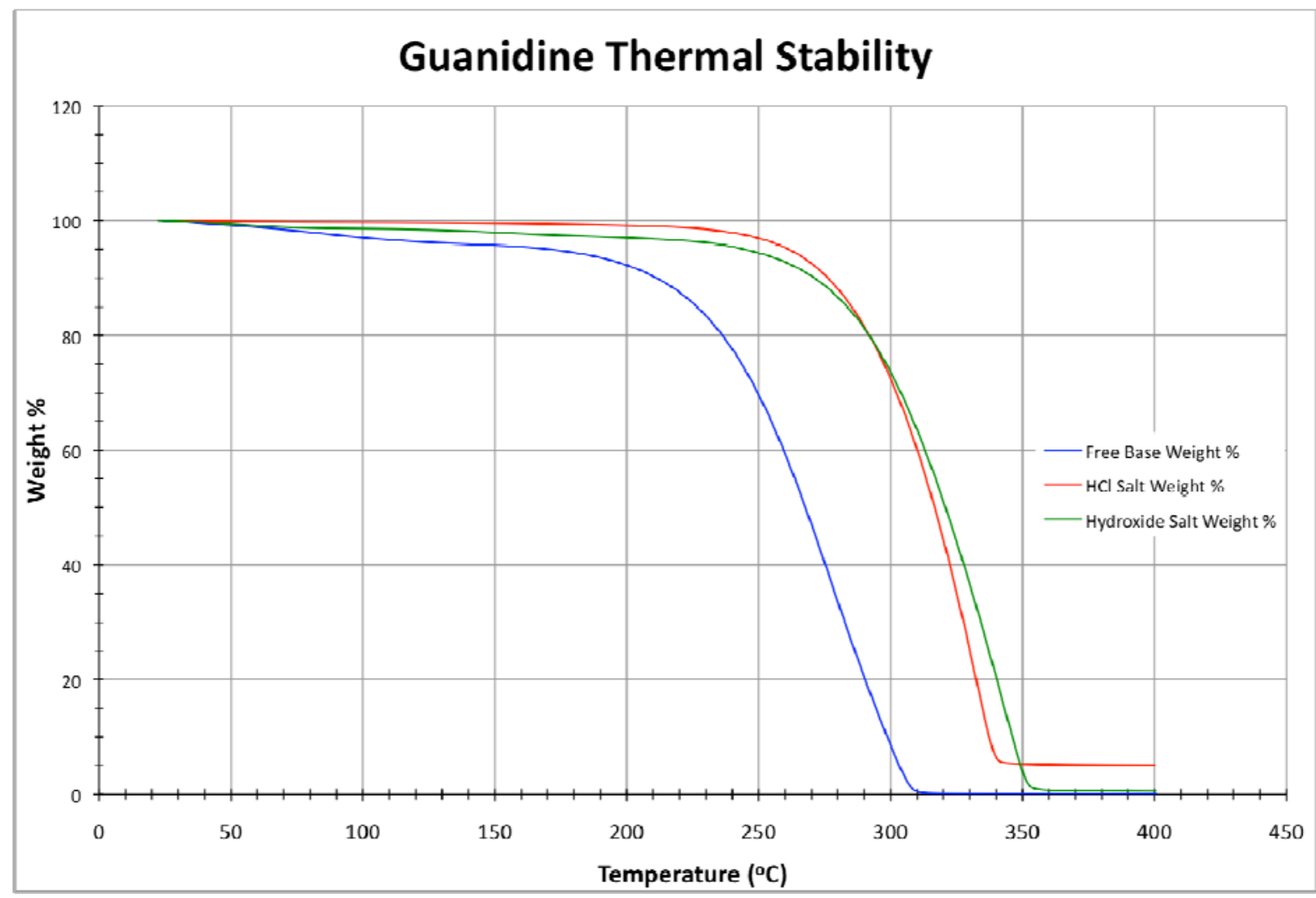

Fig. 4.3. The free base has a decomposition onset at $225^{\circ} \mathrm{C}$ while the hydroxide is at $275^{\circ} \mathrm{C}$ and hydrochloride is at $282{ }^{\circ} \mathrm{C}$. 



\section{GC METHOD DEVELOPMENT FOR ANALYSING OF DCITG}

A method for the analysis of DCiTG by GC and the detection of DCiTG by mass spectrometry and flame ionization detector (FID) is described. This chapter highlights the utility of GC for the analysis of DCiTG both in synthesis of DCiTG and in the NG-CSSX solvent mixture.

\subsection{INTRODUCTION}

DCiTG is a synthesized generic version of the active guanidine reagent in the Cognis extractant LIX $^{\circledR}$ $79^{16}$. This guanidine is a key component of the ORNL NG-CSSX process solvent ${ }^{3,11}$. In order to determine the effectiveness and purity in the synthesis of a generic version of Cognis extractant LIX 79, analytical methods are required. While NMR spectroscopy is useful to determine purity, the limit of detection for ${ }^{1} \mathrm{H}$ NMR makes the quantitation of impurities present in concentrations less than $3 \%$ difficult. GC, with a much lower limit of detection than NMR, is able to reproducibly determine the concentrations of impurities that are present in concentrations of $0.1 \%$, thus making it an attractive technique for this type of analysis. In Chapter 6, we report the use of GC as an analytical technique for the analysis of DCiTG. The general effectiveness of using GC to determine the presence of impurities in both a synthetic DCiTG mixture and the commercial Cognis LIX 79 is discussed, but details such as limits of detection, the identity of all of the impurities, and the linear response range for DCiTG were not described. In this chapter we elucidate in detail a method of analysis for DCiTG by GC, and the detection of DCiTG by MS and FID. The data from the GC/MS was used to assign most of the impurities present in the synthetic DCiTG, and provided proof that the guanidines were able to survive the GC column at $300{ }^{\circ} \mathrm{C}$ intact. Using our response factor calibrations, we were able to apply our GC method to the analysis of the NG-CSSX solvent mixture, providing a promising tool for future stability and degradation studies.

\subsection{EXPERIMENTAL SECTION}

\subsubsection{Conditions for running GC and GC/MS}

The compound was analyzed on two different instruments: an Agilent/HP 6850 GC equipped with an FID detector and an Agilent/HP 5890 GC equipped with a MS detector.

For the 6850/FID instrument, the inlet was set to split injection at a 50:1 ratio (split flow $108.2 \mathrm{~mL} / \mathrm{min}$, total flow $115.2 \mathrm{~mL} / \mathrm{min}$ ). The injection port was set at a temperature of $280^{\circ} \mathrm{C}$. The column used was an Agilent HP-5MS (5\% Phenyl methyl siloxane) open capillary column with a $30 \mathrm{~m}$ length, $0.25 \mathrm{~cm}$ inner 
diameter, and stationary-phase thickness of $0.25 \mu \mathrm{m}$. The $\mathrm{GC}$ used $\mathrm{H}_{2}$ as the carrier gas, set in constant flow mode at $2.2 \mathrm{~mL} / \mathrm{min}$ (a nominal head pressure of $14.30 \mathrm{psi}$, to give an average linear velocity of 60 $\mathrm{cm} / \mathrm{sec}$ ). The detector was set at $280{ }^{\circ} \mathrm{C}$, with an $\mathrm{H}_{2}$ flow of $50 \mathrm{~mL} / \mathrm{min}$, a compressed air flow of 450 $\mathrm{mL} / \mathrm{min}$, and no make-up gas.

For the 5890/MS instrument, the injection port was set at a temperature of $250{ }^{\circ} \mathrm{C}$. The column used was a J\&W HP-5MS (5\% Phenyl methyl siloxane) open capillary column with an approximate length of $25 \mathrm{~m}^{\mathrm{i}}$, $0.25 \mathrm{~cm}$ inner diameter, and stationary-phase thickness of $0.25 \mu \mathrm{m}$. The GC used He as the carrier gas, set in constant flow mode at $1 \mathrm{~mL} / \mathrm{min}$. The detector was set at $280^{\circ} \mathrm{C}$.

Using this set up, the product, reactants, and crude reaction mixtures were analyzed using the following temperature program: $100{ }^{\circ} \mathrm{C}$ ramped at $20{ }^{\circ} \mathrm{C} / \mathrm{min}$ to a final temperature of $300{ }^{\circ} \mathrm{C}$, then held at $300{ }^{\circ} \mathrm{C}$ for three $\mathrm{min}$. The integrator was started at $2.5 \mathrm{~min}$ and recorded signal to the end of the run.

\subsection{RESULTS AND DISCUSSION}

Because DCiTG is a complex mixture of isomer ${ }^{\mathrm{ii}}$, the limit of detection for DCiTG is much lower than that of a pure compound. Using the GC/FID instrument, a linear detector response was established for samples between $0.5 \mathrm{mg} / \mathrm{mL}(1.2 \mathrm{mM})$ and $10 \mathrm{mg} / \mathrm{mL}(24.7 \mathrm{mM})$. See Fig. 5.1. On the GC/MS instrument, the sensitivity was lower. At $0.5 \mathrm{mg} / \mathrm{mL}(1.2 \mathrm{mM})$, the DCiTG peaks could clearly be seen in the mass spectrum, but only fragment mass peaks could be identified and the mass spectrum had a low signal-to-noise $(\mathrm{S} / \mathrm{N})$ ratio $^{\mathrm{iii}}$ relative to the more concentrated samples. When the concentration was increased to $1.0 \mathrm{mg} / \mathrm{mL}(2.4 \mathrm{mM})$, the molecular ion peaks for the various isomers in the DCiTG were identifiable, while the $\mathrm{S} / \mathrm{N}$ was still much lower than could be observed on the GC/FID.

\footnotetext{
i The column in this particular instrument was of an unknown age and had been manually shortened from its original length of $30 \mathrm{~m}$. The length approximation of $25 \mathrm{~m}$ is based on the differences in retention time between the two instruments when the carrier gas and velocity are accounted for.

ii Analysis of the starting amine by GC revealed 97 isomers as individual peaks, and there are probably many more that were not individually separable.

iii The mass spectrum contained a large number of ion peaks that were consistent with column bleed, at this concentration many of the more prominent column bleed peaks were of comparable intensity to the DCiTG fragment peaks.
} 


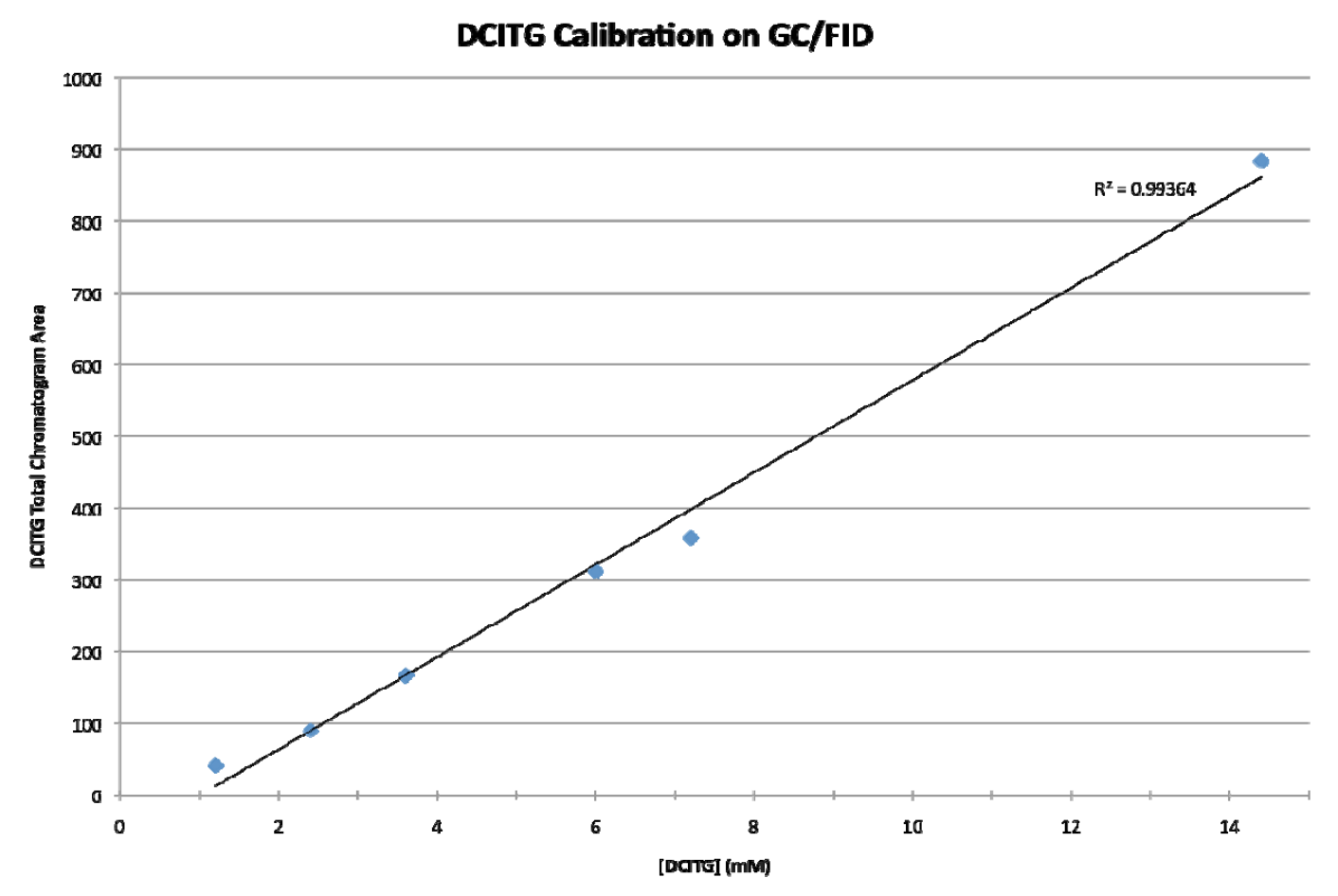

Fig. 5.1. Calibration of the response factor of DCiTG on the GC/FID Instrument.

Additionally, the GC/MS was able to provide useful data about the nature of many of the impurities in the synthetic mixture. It was noted that for the most part, the synthetic version was similar to the Cognis LIX 79 (Table 1.1). In Chapter 4 we reported that the major impurity in the generic DCiTG was dicyclohexylurea (DCU). Using GC/MS we subsequently learned that this major impurity is an additional guanidine, tricyclohexyl guanidine. The generic version still contains an unknown compound, not present in the Cognis product, that we traced to the batch of DCC used and is most likely an organophosphine oxide catalyst (Unknown 1). 
Table 5.1. Retention time and compound assignment

\begin{tabular}{|l|l|l|l|l|}
\hline $\begin{array}{l}\text { Retention } \\
\text { Time (GC) } \\
\text { MS) }\end{array}$ & $\begin{array}{l}\text { Retention } \\
\text { Time (GC) } \\
\text { FID) }\end{array}$ & Compound & $\begin{array}{l}\text { Percent } \\
\text { composition } \\
\text { in synthetic } \\
\text { mixture }\end{array}$ & $\begin{array}{l}\text { Percent } \\
\text { composition } \\
\text { in Cognis } \\
\text { mixture }\end{array}$ \\
\hline $2.5-3.5$ & $3.0-4.0$ & Amines & $0 \%$ & $0 \%$ \\
\hline 4.90 & 4.97 & DCC & $.08 \%$ & $.03 \%$ \\
\hline 4.95 & 5.05 & Compound 1 & $.03 \%$ & $0 \%$ \\
\hline 6.50 & 6.62 & DCU & $.17 \%$ & $.25 \%$ \\
\hline 6.72 & 6.77 & Unknown 1 & $.30 \%$ & $0 \%$ \\
\hline 7.69 & 7.75 & TCG & $2.30 \%$ & $.22 \%$ \\
\hline $8.25-10.25$ & $8.25-10.25$ & DCITG isomers & $>97 \%$ & $>99 \%$ \\
\hline
\end{tabular}

\subsubsection{Mass Spectra of DCiTG isomers}

The average mass spectrum for the total DCiTG peak shows molecular ion peaks for DCiTG isomers with 12-15 carbon chain lengths $(\mathrm{M}+=391,405,419,433)$ (Fig. 5.3). By selecting specific regions inside the total ion chromatogram of DCiTG, individual mass spectra for the DCiTG isomers with 12, 13, and 14 carbon chain lengths can be isolated from the average mass spectrum (Figs. 5.4, 5.5, 5.6). 


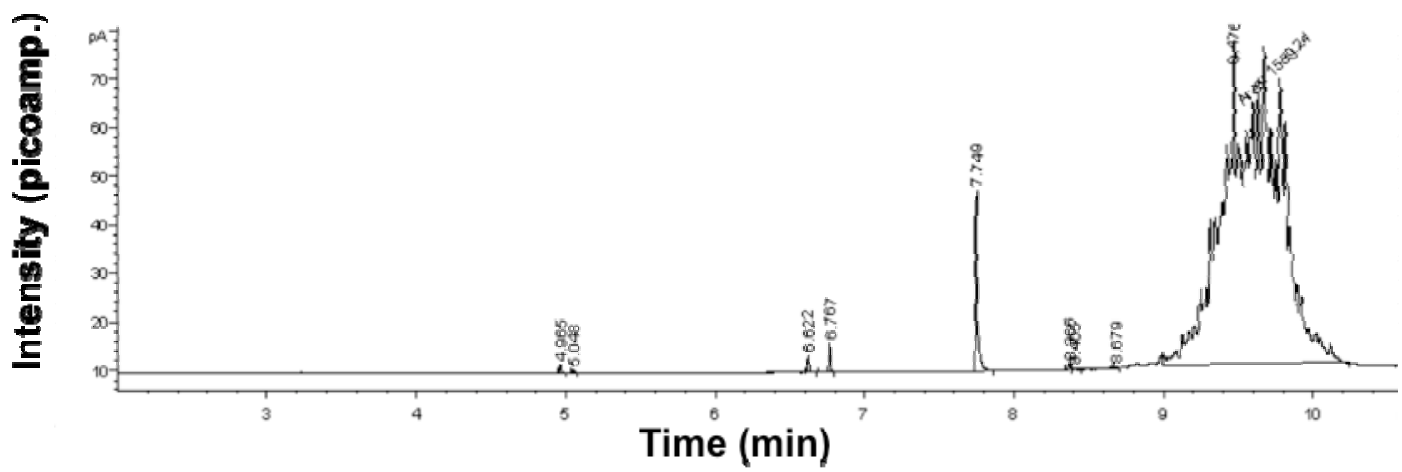

GCFFID $100 \rightarrow 300$ 20 $20 \mathrm{~cm}$ In (hold $3 \mathrm{mln}$ ), H2 carrier, Agllent HP-5MS column, $30 \mathrm{~m}$

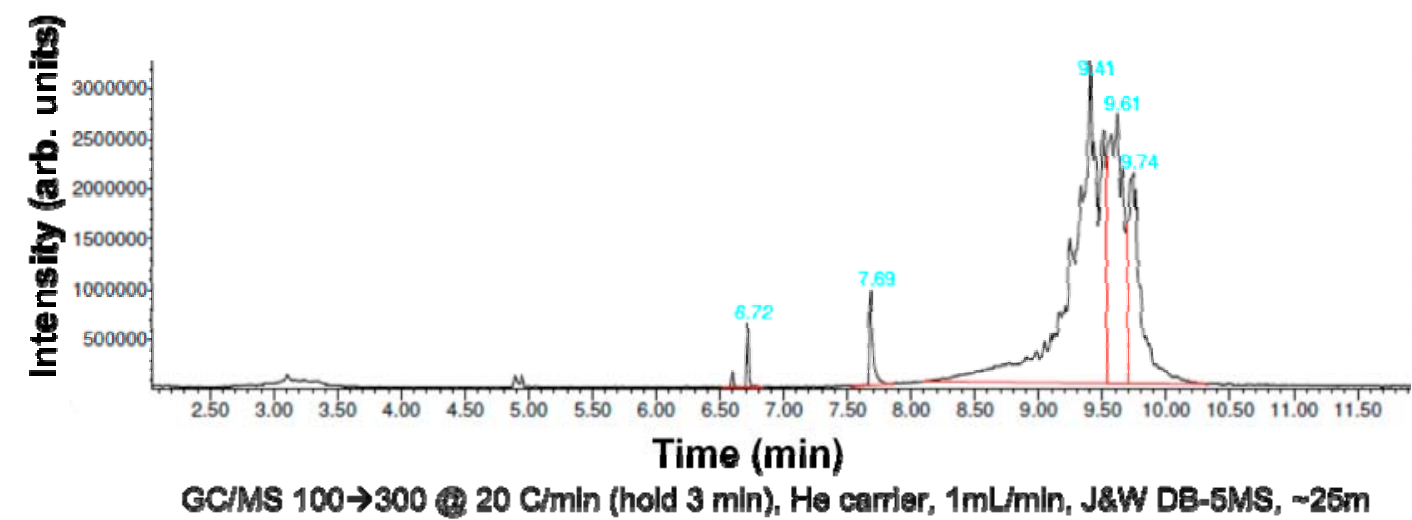

Fig. 5.2. Chromatograms from both instruments of synthetic DCiTG mixtures.

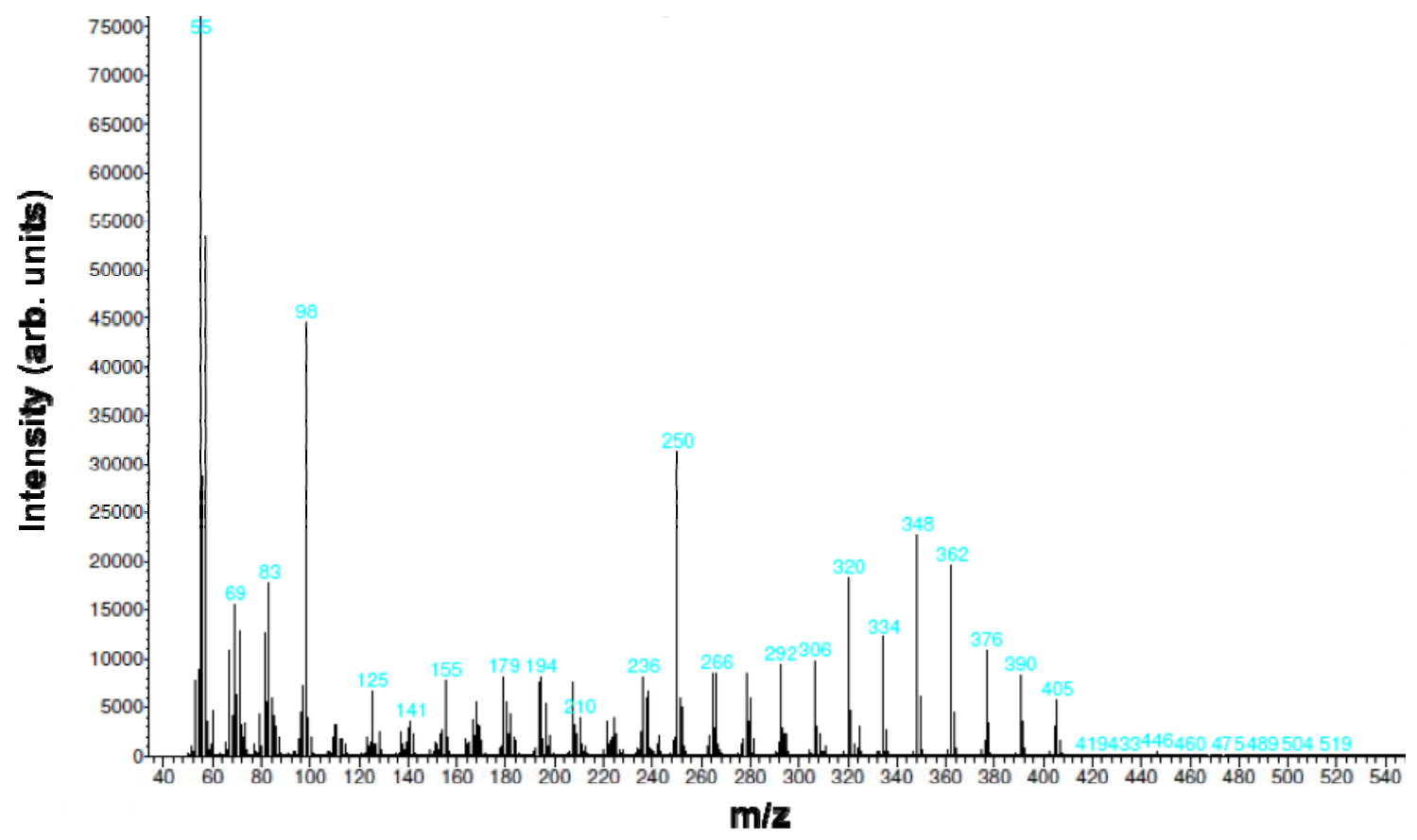

Fig. 5.3. Average mass spectrum of DCiTG peaks 


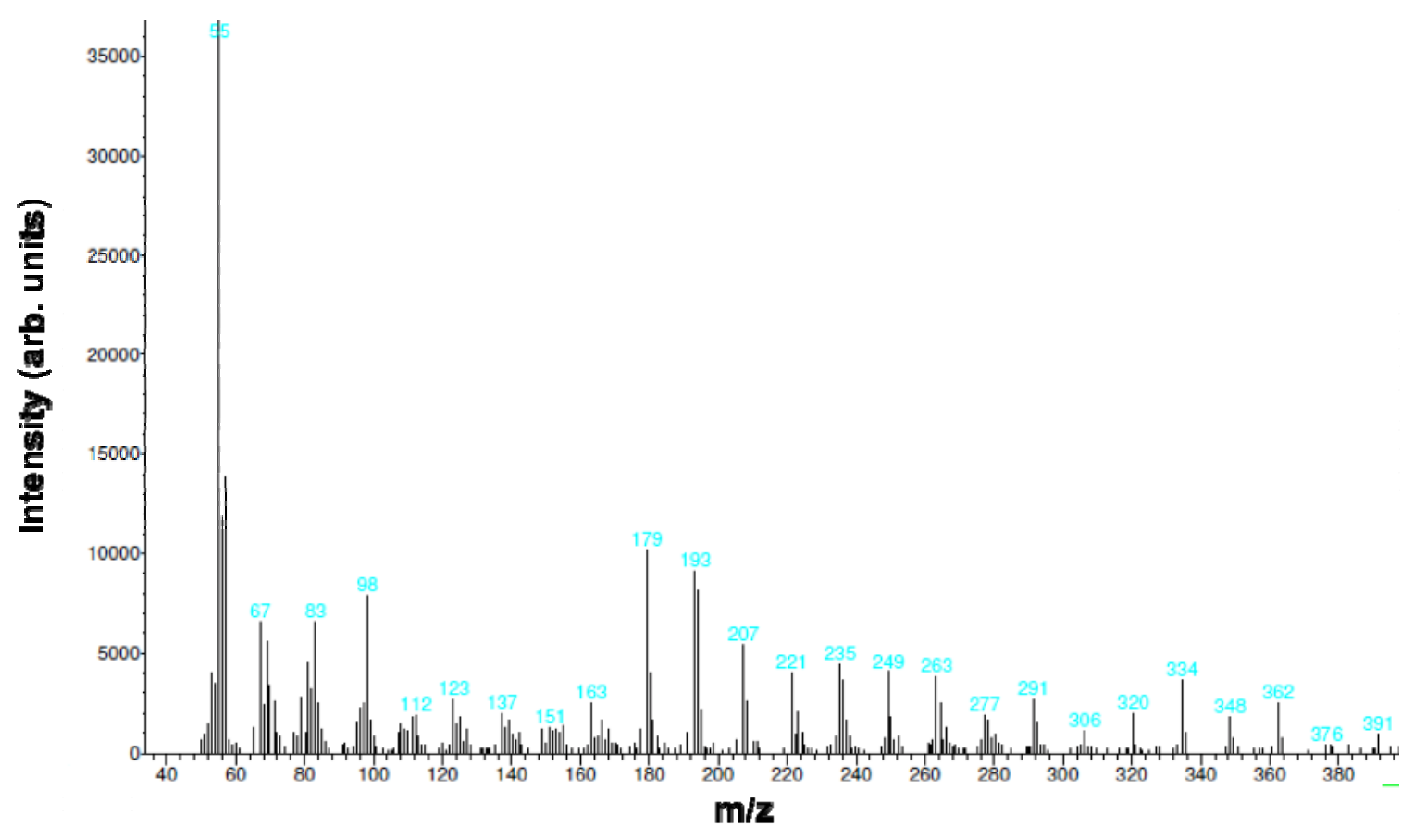

Fig. 5.4. Mass spectrum for 12 carbon chain DCiTG isomer.

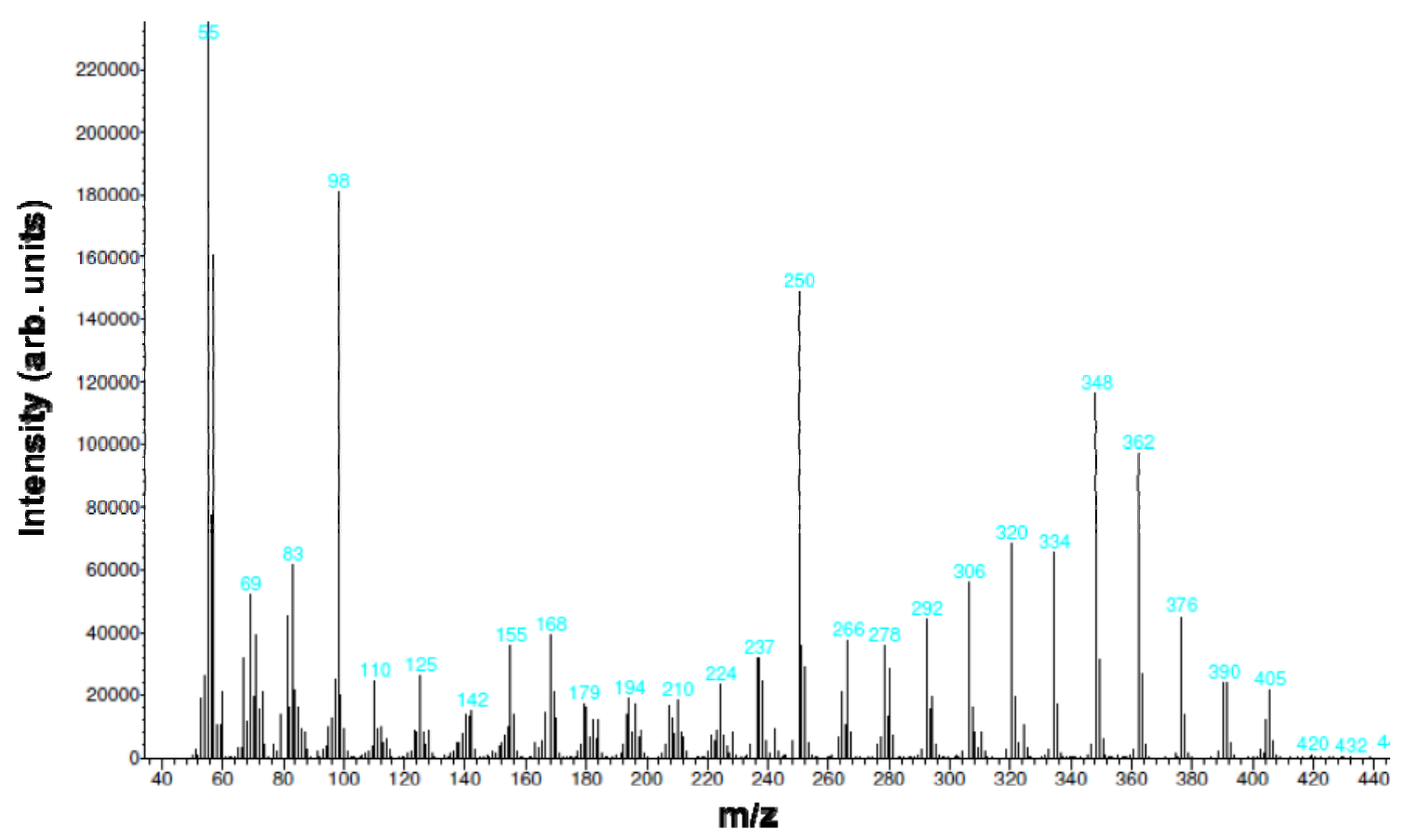

Fig. 5.5. Mass spectrum for 13-carbon chain DCiTG isomer. 


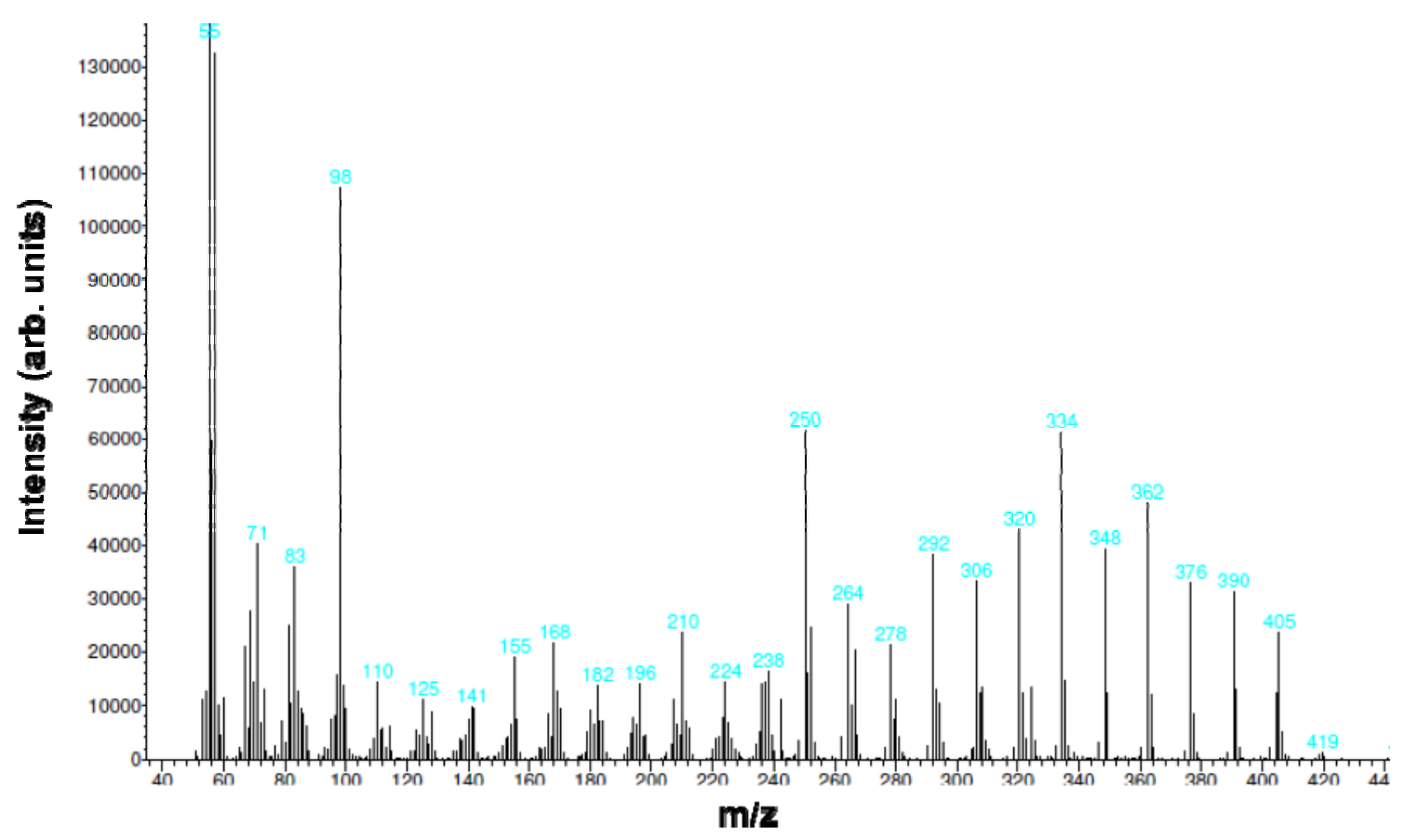

Fig. 5.6. Mass spectrum for 14-carbon chain DCiTG isomer.

As expected, from the average mass spectrum it appears that the 13-carbon chain DCiTG isomers are the most prevalent. This also provides evidence that the DCiTG isomers are able to survive the GC even though the oven temperature, injection port temperature, and detector temperature all exceed the thermal decomposition onset temperature of $225^{\circ} \mathrm{C}$.

\subsubsection{GC as it applies to the NG-CSSX solvent}

Since the concentration of DCiTG in the NG-CSSX solvent is $3 \mathrm{mM}$, GC may be a good way to evaluate the loss/degradation of DCiTG in the solvent mixture since this concentration is above our useful limit of detection of $1.2 \mathrm{mM}$. In a sample test, the solvent mixture was injected into the GC/FID instrument using the same temperature program except that the solvent delay was set at $3 \mathrm{~min}$ to allow all of the $\operatorname{Isopar}^{\circledR} \mathrm{L}$ solvent to escape before data collection (Fig. 5.7). 


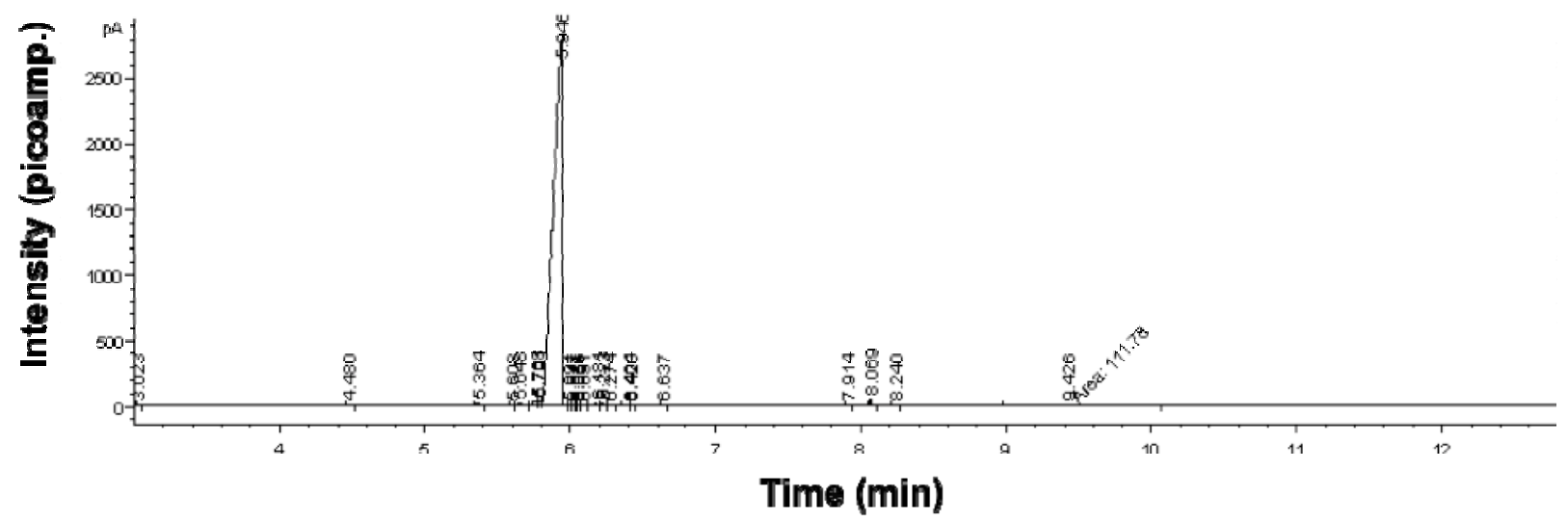

Fig. 5.7. GC chromatogram of NG-CSSX solvent.

As can be seen, the DCiTG (at $9.4 \mathrm{~min}$ ) is dwarfed by the much higher concentration solvent modifier (Cs7 $\mathrm{SB}$ ) at $5.95 \mathrm{~min}$. When the guanidine region is expanded, the DCiTG is clearly present (Fig. 5.8).

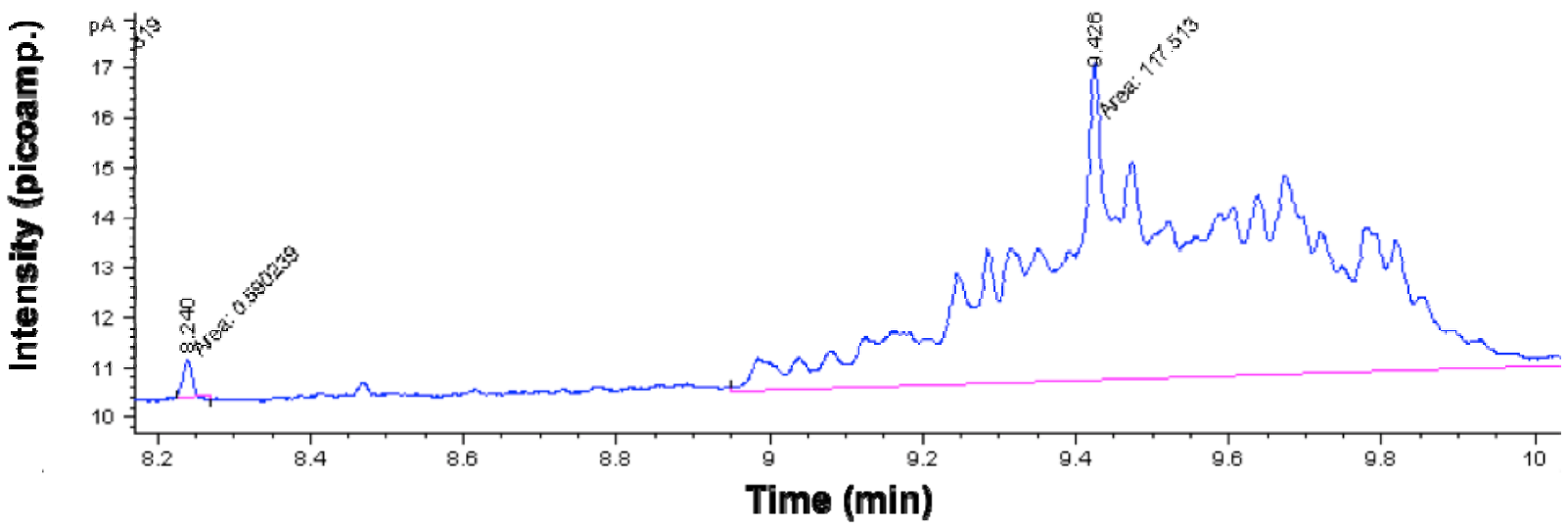

Fig. 5.8. DCiTG guanidine region in NG-CSSX solvent mixture (DCiTG conc $3 \mathrm{mM}$, area $=117.5$ )

This response is comparable to what was observed in the chromatograms of DCiTG at $2.4 \mathrm{mM}$ and 3.6 $\mathrm{mM}$ used in generating the FID calibration curve (Fig. 5.9). 

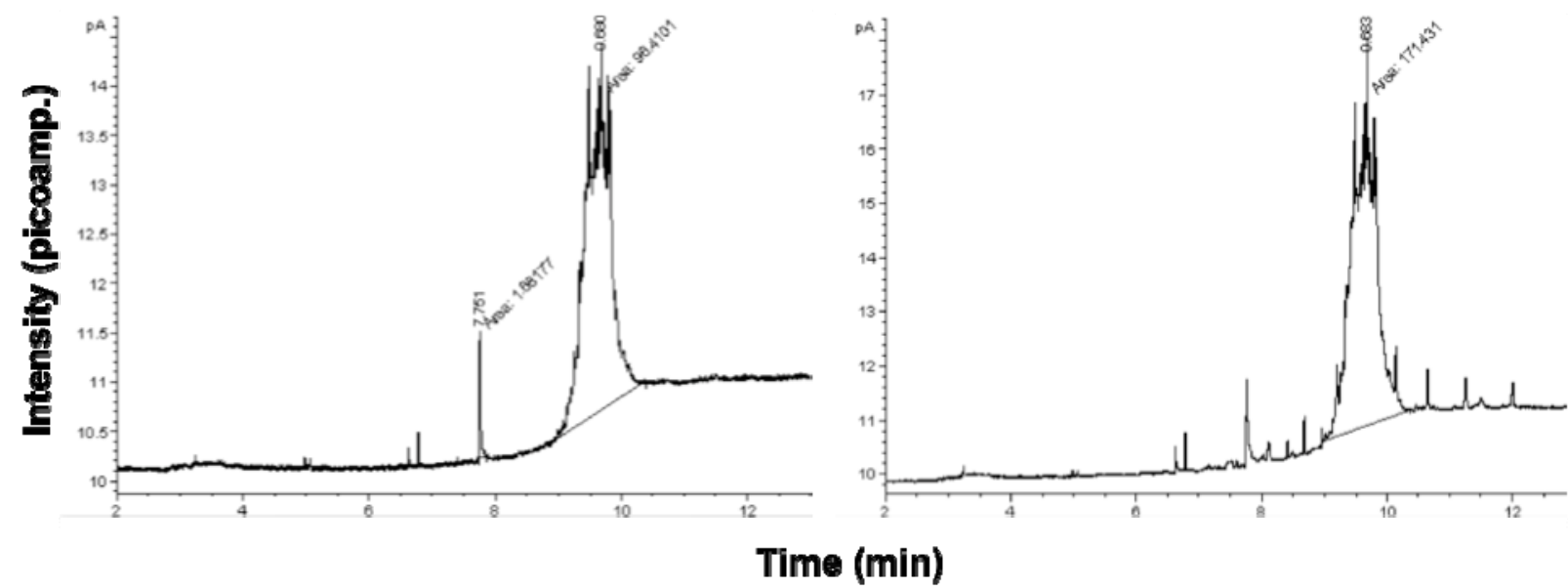

Fig. 5.9. DCiTG calibration chromatograms (DCiTG conc $2.4 \mathrm{mM}$ and $3.6 \mathrm{mM}$, respectively)

When the area for DCiTG at $3 \mathrm{mM}$ is inserted into Fig. 5.1, it is seen that it fits as expected with the response factor calibration (Fig. 5.10).

\section{DCITG Calibration on GC/FID}

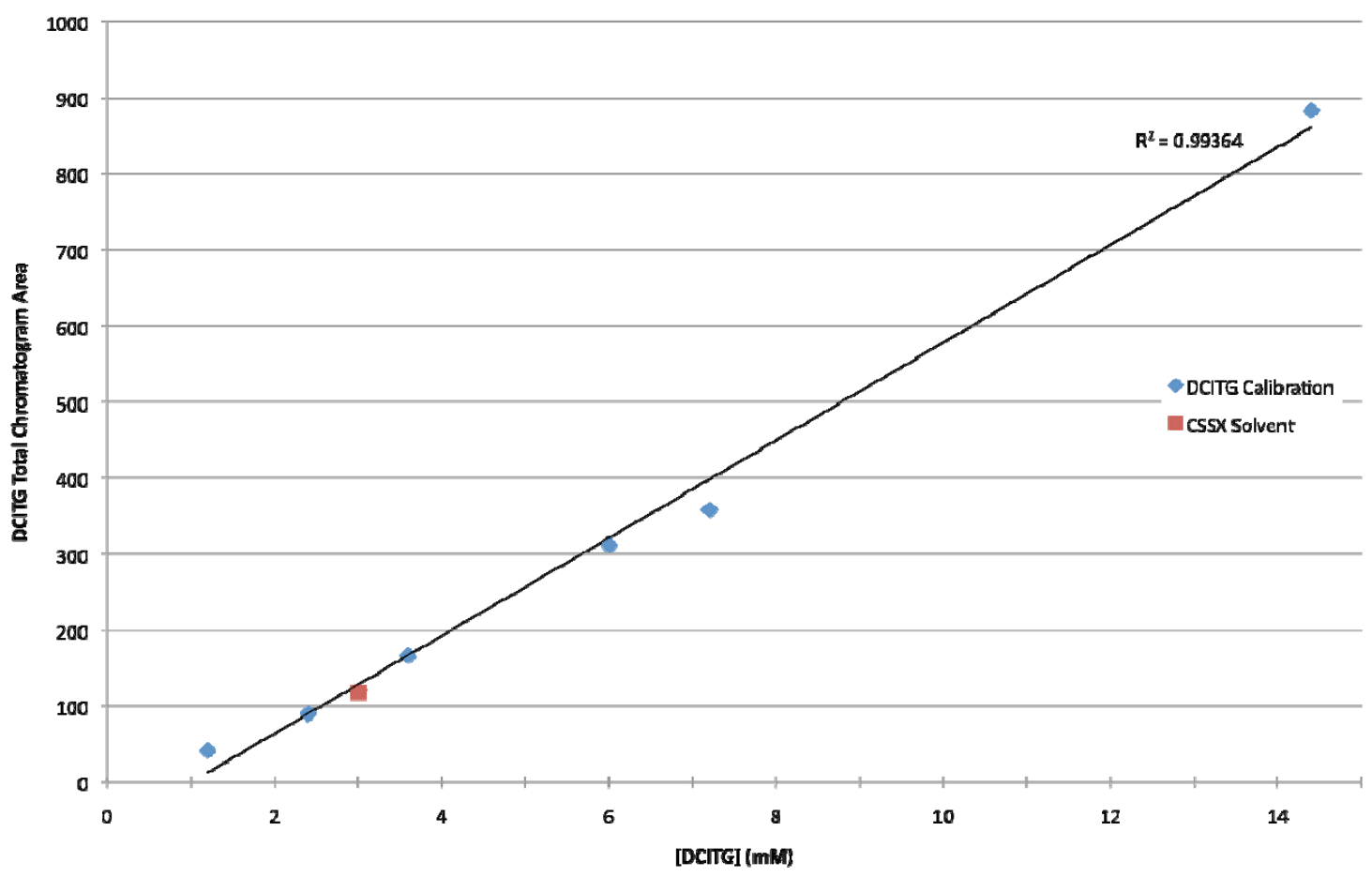

Fig. 5.10. Comparison of the detector response for DCiTG in NG-CSSX solvent with the calibration.

This result provides evidence that GC may be a promising tool in the evaluation of the NG-CSSX solvent as it relates to the stability and degradation of the DCiTG. 


\subsection{SUMMARY}

A method for the analysis of DCiTG by GC has been developed to detect and quantify DCiTG both in standard solutions and in the fully composed NG-CSSX solvent mixture. The GC/MS data confirm that DCiTG itself, and not degradation by-products of DCiTG are being detected. The linear detector response range established from the calibration curve indicates that most of the DCiTG is surviving the GC conditions and that more than just trace amounts of what is being injected are being detected. The application of the method has been used as a useful aid in the synthesis of DCiTG as it provides a quick and reproducible quantitation of the impurities present in the DCiTG. Further investigation is being conducted into the application of this method as it relates to the stability and fate of DCiTG in the fully composed NG-CSSX solvent mixture. 


\section{MINIMUM PURITY REQUIREMENTS FOR MAXCALIX, DCITG AND DCITG•HCL SUPPRESSOR FOR NG-CSSX SOLVENT}

\subsection{INTRODUCTION}

This chapter describes the minimum purity requirements for 1,3-alt-25,27-bis(3,7-dimethyloctyl-1oxy)calix[4]arenebenzocrown-6 (MaxCalix), DCiTG, and the hydrochloride salt DCiTG•HCl.

\subsection{RECOMMENDATIONS}

\subsubsection{MaxCalix}

This material is synthesized using the two major steps of first alkylating calix[4]arene to produce 25,27-di(3,7-dimethyloctyl-1-oxy)calix[4]arene, followed by the "crowning step" to produce the title product. Impurities can arise from incomplete reactions. Table 6.1 specifies the minimum required purity of MaxCalix (to ensure satisfactory performance in the NG-CSSX Solvent), and the minimum allowable amounts of various impurities, intermediates, and starting materials, that can be present in the final MaxCalix product. The purity can be assayed by proton and carbon NMR, thin Layer chromatography, and $\mathrm{C}, \mathrm{H}$ combustion (elemental) analysis (agreement between calculated and analyzed should be within $0.4 \%$.). A melting point (range) could be provided, however calixarene-crown ethers often do not possess sharp crystalline melting points, and hence melting point may not be an adequate indicator of purity for this compound.

Table 6.1 MaxCalix product specifications summary

\begin{tabular}{lc|}
\hline \multicolumn{1}{|c|}{ Component } & Allowable range (mole \%) \\
\hline MaxCalix & $>97 \%$ \\
Polymer impurities & $<3 \%$ \\
Calix[4]arene & $<0.1 \%$ \\
25,27-di(3,7-dimethyloctyl-1-oxy)calix[4] arene & $<0.1 \%$ \\
All other polyethers and podands & $<0.1 \%$ \\
\hline
\end{tabular}

\subsubsection{1,3-dicyclohexyl-2-(isotridecyl)guanidine (DCiTG)}

"DCiTG" is a synthesized generic version of the active guanidine reagent in the Cognis extractant LIX ${ }^{\circledR}$ $79^{16}$. This guanidine is a key component of the ORNL NG-CSSX process solvent ${ }^{3,11}$. The guanidine as the free base is physically a viscous oil, which can be difficult to work with when preparing the NG-CSSX solvent. However, the hydrochloride form $(\mathrm{DCiTG} \cdot \mathrm{HCl})$, is a white to off-white solid that is easier to work with. Hence, DCiTG $\bullet \mathrm{HCl}$ is presently considered on this basis to be the preferred form for use in preparing the NG-CSSX solvent. We have prepared NG-CSSX solvent with both free-base and $\mathrm{HCl}$ 
forms with no noticeable differences in performance, which follows from the expectation that the $\mathrm{HCl}$ form is neutralized on contact with alkaline waste. It is not known, however, whether the two forms differ in shelf life or other properties in a way that could in the future alter our current preference for the $\mathrm{HCl}$ form. The structures of DCiTG and DCiTG $\bullet \mathrm{HCl}$ are shown in Fig. 6.1 below.

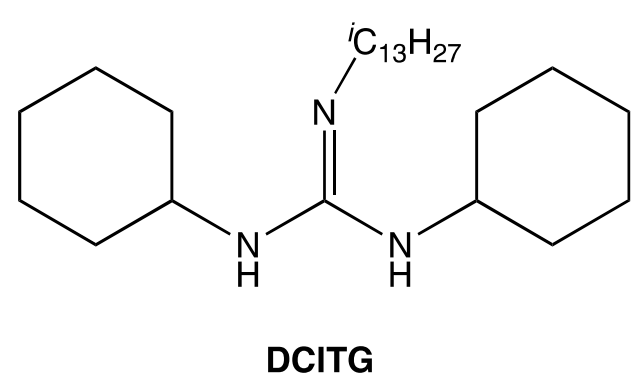

Properties: Viscous Amber Oil Nominal Chemical Formula: $\mathrm{C}_{26} \mathrm{H}_{51} \mathrm{~N}_{3}$ Nominal Molecular Weight: 405.70

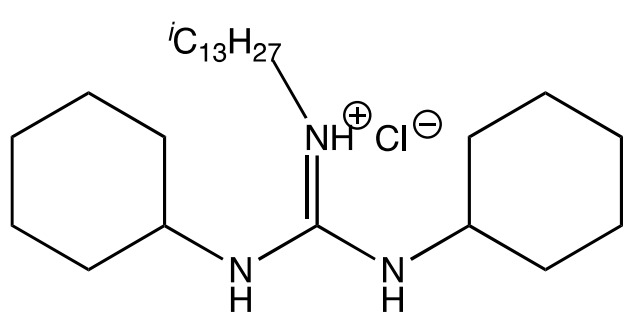

$\mathrm{DCITG} \cdot \mathrm{HCl}$

Properties: White/off-white hydroscopic solid Nominal Chemical Formula: $\mathrm{C}_{26} \mathrm{H}_{52} \mathrm{~N}_{3} \mathrm{Cl}$ Nominal Molecular Weight: 442.16

Fig. 6.1. Structure and properties of DCiTG and DCiTG•HCI.

The guanidine can be prepared by the reaction of DCC with iTDA ${ }^{16}$, wherein the iTDA (CAS No. 3572381-0) is not a pure material but actually a complex mixture of branched C10-C14 amines, with typically the greatest fraction (85-90\%) being the $\mathrm{C} 11-\mathrm{C} 13$ chain lengths. The boiling point range of this mixture is typically $270-295{ }^{\circ} \mathrm{C}$ at 760 torr. Since the amine is marketed as iTDA, with nominal molecular formula and weight of, respectively, $\mathrm{C}_{13} \mathrm{H}_{29} \mathrm{~N}$ and $199.37 \mathrm{~g} / \mathrm{mol}$, we will designate the DCiTG as having "nominal" molecular formula and weight of, respectively, $\mathrm{C}_{26} \mathrm{H}_{51} \mathrm{~N}_{3}$ and $405.70 \mathrm{~g} / \mathrm{mol}$. Accordingly, the hydrochloride salt, formed by the reaction of DCiTG with the stoichiometric amount of hydrochloric acid, will be assigned the nominal molecular formula and weight of, respectively, $\mathrm{C}_{26} \mathrm{H}_{52} \mathrm{~N}_{3} \mathrm{Cl}$ and 442.16 $\mathrm{g} / \mathrm{mol}$.

Impurities can arise from incomplete reaction between the amine and DCC. Also, some N,N'dicyclohexylurea (DCU) can be formed by reaction of DCC with adventitious water, and possibly from thermal decomposition of DCiTG. Accordingly, the most common impurities in DCiTG will most likely be iTDA (mixture), DCC, and DCU. The effect of these impurities on NG-CSSX solvent performance is currently under investigation, but it is thought on the basis of their structures that these impurities will either be benign (DCU) or wash out of the solvent during processing (amines, DCC). The Tables below specify the minimum required purity of DCiTG and DCiTG $\cdot \mathrm{HCl}$ (to ensure satisfactory performance in the NG-CSSX Solvent), and the minimum allowable amounts of unreacted starting materials and side 
products that can be present, consistent with information currently available. The purity of DCiTG can be assayed by proton and carbon NMR, thin Layer chromatography, and GC. Since DCiTG $\bullet H C l$ would be prepared from DCiTG, following the analysis of the parent DCiTG, the purity of DCiTG• $\mathrm{HCl}$ can be checked can be by proton and carbon NMR, and also possibly by titration (neutralization equivalent).

Table 6.2. DCiTG product specifications summary

\begin{tabular}{lc}
\hline \multicolumn{1}{c}{ Component } & Allowable range (mole \%) \\
\hline 1,3-dicyclohexyl-2-(isotridecyl)guanidine (DCiTG) & $>95 \%$ \\
N,N'-Dicyclohexylurea (DCU) & $<3 \%$ \\
N,N'-Dicyclohexylcarbodiimide (DCC) & $<0.5 \%$ \\
Isotridecylamine mixture & $<1 \%$ \\
All other impurities & $<0.5 \%$ \\
\hline
\end{tabular}

Table 6.3. DCiTG•HC1 product specifications summary

\begin{tabular}{lc}
\hline \multicolumn{1}{c}{ Component } & Allowable range (mole \%) \\
\hline 1,3-dicyclohexyl-2-(isotridecyl)guanidine hydrochloride & \\
$($ DCiTG $H C l)$ & $>95 \%$ \\
N,N'-Dicyclohexylurea (DCU) & $<3 \%$ \\
N,N'-Dicyclohexylcarbodiimide (DCC) & $<0.5 \%$ \\
Isotridecylamine hydrochloride mixture & $<1 \%$ \\
All other impurities & $<0.5 \%$ \\
\hline
\end{tabular}

GC is a satisfactory method for analyzing DCiTG. It is recommended that samples be prepared under a nitrogen atmosphere (to exclude carbon dioxide) at concentrations of $0.5-1.0 \mathrm{mg} / \mathrm{mL}$ in dichloromethane. For example, what follows is a description of the GC analysis of a sample of DCiTG from Cognis, using an Agilent/HP 6850 GC equipped with an FID detector. General operating conditions were as described in section 5.2.1. Using this set up, the product, reactants, and crude reaction mixtures were analyzed using the temperature program: $100{ }^{\circ} \mathrm{C}$ held for $2.5 \mathrm{~min}$, ramp of $20{ }^{\circ} \mathrm{C} / \mathrm{min}$ to a final temperature of $300{ }^{\circ} \mathrm{C}$, then held at $300{ }^{\circ} \mathrm{C}$ for $1.5 \mathrm{~min}$ (see Fig. 6.2). The integrator was started at $2.5 \mathrm{~min}$ and recorded signal to the end of the run. 


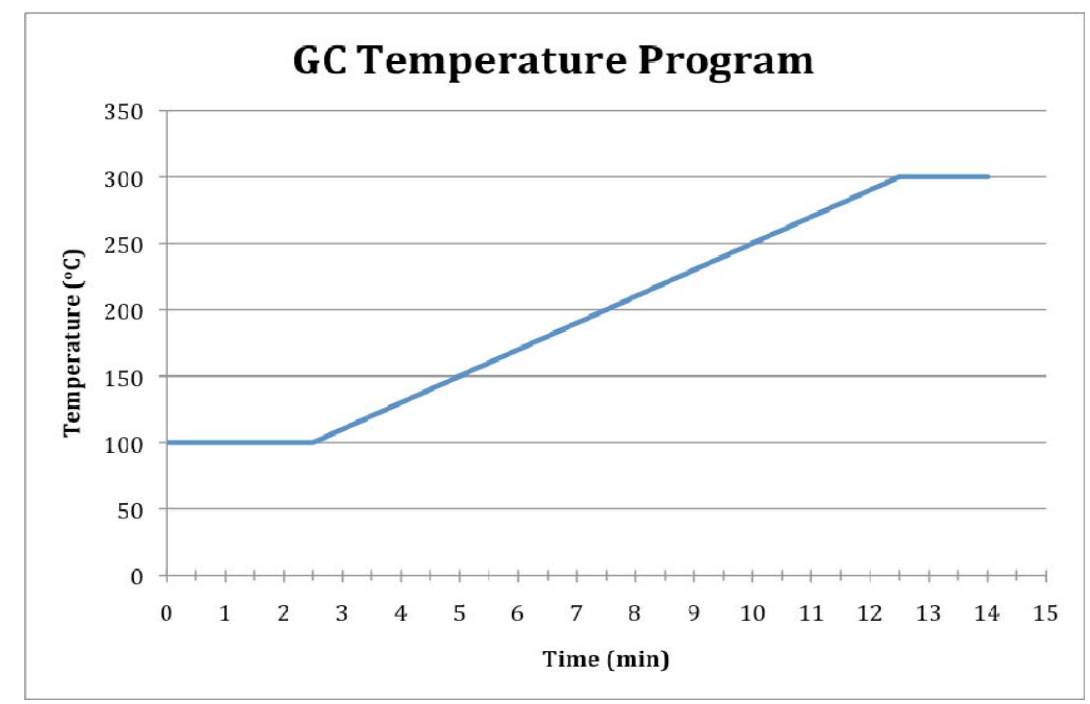

Fig. 6.2. Graphic of GC oven temperature program conditions for analyzing DCiTG.

Below in Fig. 6.3 is the chromatogram, showing the DCiTG eluting as a very broad peak spanning 11.12 to 12.62 minutes using these conditions. The retention times of the iTDA mixture, DCC, and DCU were independently measured using authentic standards (see Table 6.4). In this sample of DCiTG, minor amounts of unreacted DCC, and side product DCU are observed, at 0.03 and $0.22 \%$, respectively. No residual iTDA was observed. There are two additional peaks due to unknown components that together are present at $0.25 \%$. The purity of this sample of DCiTG is thus $>99 \%$.

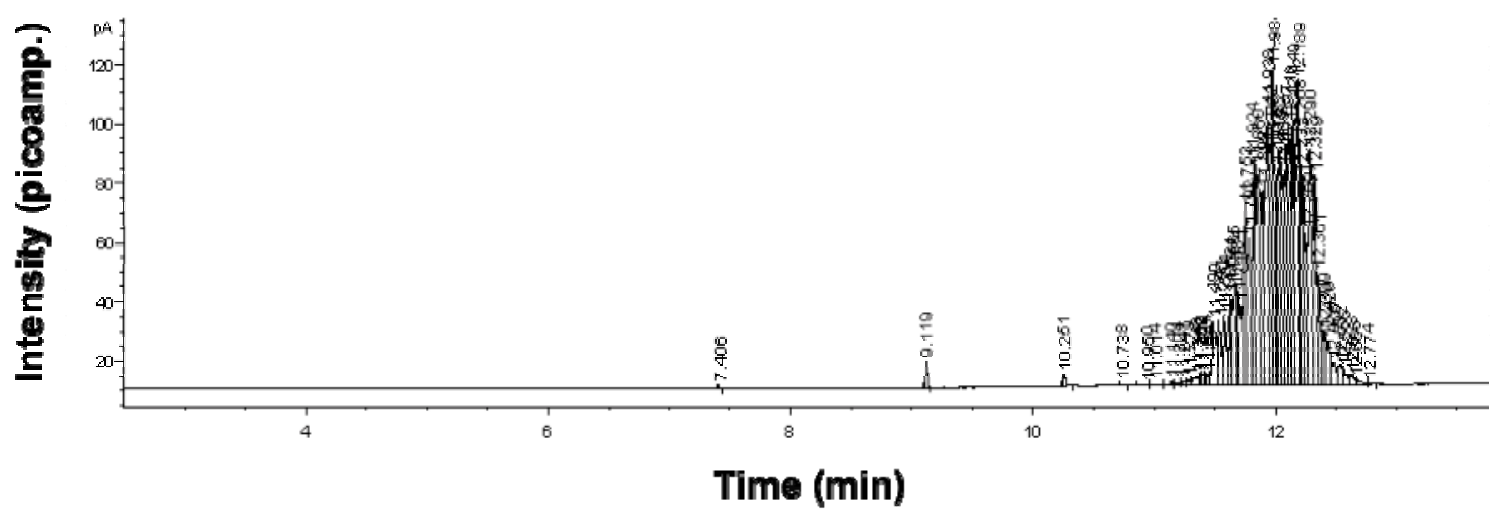

Fig. 6.3. Chromatogram of commercial sample of DCiTG (LIX 79 component) from Cognis. This sample contained $0.22 \%$ DCU, $0.03 \%$ DCC, and $0.25 \%$ of "unknown" species, with the DCiTG mixture comprising $>99 \%$.

Table 6.4 below also reiterates the allowable percentage of each component, where the unknown species would fall under the "All other impurities" category of Table 6.2. 
Table 6.4. Retention times for compounds that may be present in DCiTG

\begin{tabular}{lcc}
\hline \multicolumn{1}{c}{ Compound } & $\begin{array}{c}\text { Retention time } \\
(\text { min) }\end{array}$ & Allowable Percentage \\
\hline Isotridecylamine mixture & $4.80-5.83$ & $<1 \%$ \\
N,N'-Dicyclohexylcarbodiimide (DCC) & 7.41 & $<0.5 \%$ \\
Unknown 1 & 9.12 & Combined $<0.5 \%$ \\
Unknown 2 & 9.25 & Combined $<0.5 \%$ \\
N,N'-Dicyclohexylurea (DCU) & 10.25 & $<3 \%$ \\
DCiTG mixture & $11.12-12.62$ & $>95 \%$ \\
\hline
\end{tabular}





\section{EMPIRICAL MODEL FOR THE NG-CSSX SOLVENT}

\subsection{INTRODUCTION}

In this chapter, an empirical model is presented to predict $D_{C S}$ values for the next-generation solvent over a range of SRS salt-waste compositions. A model is needed so that the impact of changes in the waste composition on flowsheet performance can be estimated for the purposes of process control and optimization as well as for assurance that conditions leading to off-normal performance can be avoided. Previously, real-waste compositions were calculated with reasonable precision $( \pm 16 \%)$ using a computer model of $D_{C S}$ developed based on the SXFIT code ${ }^{18}$ studies for the original BOBCalixC6 CSSX process $^{7,19}$. More recently ${ }^{11}$, modeling of solvent-extraction data was carried out using the computer

program $\mathrm{SXLSQI}^{20}$, a mass-action equilibrium-analysis program used to determine log $\mathrm{K}$ values corresponding to postulated species in liquid Liquid equilibria. Here we report the application of a simpler, yet still accurate, model utilising the solver function in the Microsoft Excel program. By defining the four primary species in the system likely to have the greatest effect on the $D_{C s}$ values, MaxCalix, $\mathrm{K}^{+}$, $\mathrm{OH}^{-}$, and $\mathrm{NO}_{3}{ }^{-}$, it has been possible to define an empirical equation that can predict $D_{C s}$ values based on their concentrations.

\subsection{EXPERIMENTAL SECTION}

\subsubsection{Materials}

\subsubsection{Aqueous solutions}

All solutions contained $1.00 \times 10^{-4} \mathrm{M} \mathrm{CsNO}_{3}$ (Acros Organics 99.99\%). Solutions contained $\mathrm{K}^{+}$(added in the form of $\mathrm{KOH}$, Certified ACS, Fisher Scientific) in increasing increments of $0.01 \mathrm{M}$ starting at $0.00 \mathrm{M}$ until reaching $0.10 \mathrm{M}$. A series of tests was also carried out at $0 \mathrm{M} \mathrm{K}^{+}$, with increasing amounts of $\mathrm{OH}^{-}$ concentration. Solutions contained $\mathrm{OH}^{-}$(in the form of $\mathrm{NaOH}$, Certified ACS, Fisher Scientific) in increasing increments of $0.50 \mathrm{M}$ starting at $1.00 \mathrm{M}$ until reaching $5.00 \mathrm{M}$. The total ionic strength was balanced using $\mathrm{NaNO}_{3}$ (Certified ACS, Fisher Scientific) until the $\mathrm{Na}^{+}$concentration was $5.60 \mathrm{M}$ in every solution. Each solution was stored in a $25 \mathrm{~mL}$ polyethylene bottle until extraction data was gathered. All aqueous solutions were prepared using $>18 \mathrm{MOhm} \mathrm{cm}$ at $25{ }^{\circ} \mathrm{C}$ reverse osmosis deionized water. Salt solutions were prepared using ACS reagent grade compounds (dried) and clean volumetric glassware. 


\subsubsection{Solvent components}

Solvent components were obtained from commercial sources and judged to be of adequate purity for use as received. 1-(2,2,3,3-Tetrafluoropropoxy),-3-[4-(sec-butyl)phenoxy]-2-propanol (Cs-7SB modifier) and MaxCalix were obtained from Marshallton Research Laboratories, and Isopar $^{\circledR}$ L was obtained from ExxonMobil. Solvents were prepared by weighing appropriate amounts of extractant, modifier, and suppressor into volumetric flasks and diluting with Isopar L to the mark. The components are shown in Table 7.1, except that the suppressor was not used.

Table 7.1 NG-CSSX solvent components

\begin{tabular}{|c|c|c|c|}
\hline Component & Code & Chemical Name & Structure \\
\hline Extractant & MaxCalix & $\begin{array}{l}\text { 1,3- alt-25,27-bis(3,7- } \\
\text { dimethyloctyl-1-oxy) } \\
\text { calix[4]arene- } \\
\text { benzocrown-6 }\end{array}$ & \\
\hline Modifier & Cs-7SB & $\begin{array}{l}\text { 1-(2,2,3,3- } \\
\text { Tetrafluoropropoxy)-3- } \\
\text { (4-sec-butylphenoxy)- } \\
\text { 2-propanol }\end{array}$ & \\
\hline Diluent & Isopar L & $\begin{array}{l}\mathrm{C}_{12} \text {-isoparaffinic } \\
\text { hydrocarbon }\end{array}$ & \\
\hline
\end{tabular}

\subsubsection{Methods}

Sample solutions were contacted with NG-CSSX solvent containing 0.05 M MaxCalix (lot\# 76-284-1) and $0.50 \mathrm{M}$ Cs-7SB (lot\# MOD 2010-M3) diluted in Isopar L (ExxonMobil Chemical Co. Lot\#US67377C). The DCiTG suppressor was not used as samples were only contacted for the extraction step of the extraction, scrub, and strip (ESS) process, $D_{C s}$ values for extraction have been shown to be virtually unaffected by DCiTG concentration ${ }^{21}$. The $\mathrm{D}_{\mathrm{Cs}}$ were measured using a tracer technique. Equal volumes of the required aqueous phase spiked with ${ }^{137} \mathrm{Cs}$ (Isotope Products Laboratories) were equilibrated with the organic phase. The samples were vortexed for five minutes and then equilibrated at $25{ }^{\circ} \mathrm{C}$ for fifteen minutes. This procedure was repeated and after the second equilibration the samples were centrifuged and the phases separated. This procedure was determined to be sufficient for equilibrium to be established. Two counting samples were pulled from each phase and a Packard Cobra II Autogamma radiation counter was used to analyze the samples. Samples were corrected for background and the $D_{\mathrm{Cs}}$ was calculated using the following equation:

$$
D_{C s}=\frac{\left[\mathrm{Cs}^{+}\right]_{\text {organic }}}{\left[\mathrm{Cs}^{+}\right]_{\text {aqueous }}}=\frac{\text { net } \mathrm{Cpm}_{\text {organic }}}{\text { net } \mathrm{Cpm}_{\text {aqueous }}}
$$


Accountability for each sample was checked by calculating a \% recovery based on an assay of the aqueous phase before contact. Samples with a \% recovery outside of $100 \pm 10 \%$ were discarded. All samples were run in duplicate and values calculated using Microsoft ${ }^{\circledR}$ Excel.

All procedures and equipment used in sample preparation and analysis were based on best practices for quality assurance and calibration methods ${ }^{22}$. The Denver Instrument Co. A-160 analytical balance was calibrated using standard Class 1 masses and was found to be precise within $\pm 0.05 \mathrm{mg}$. All pipettes were calibrated by the mass determination of water delivered and found to be within tolerances (rel. \% accuracy \pm 0.8 ) as specified by Rainin Instrument Co. The Cobra II Auto-gamma counter with a 3" $\mathrm{NaI}(\mathrm{Tl})$ crystal was calibrated each week the system was in use. The calibration protocol used a ${ }^{137} \mathrm{Cs}$ standard to measure the \% resolution, calibrate the high voltage, do a chi-square test of the system and measure the background over the entire energy range. The crystal had a resolution of $8 \%$ and the chisquare value was always $<25$, which was in the $99 \%$ confidence range indicating the system was stable.

\subsection{RESULTS AND DISCUSSION}

An empirical equation for the estimation of $D_{\mathrm{Cs}}$ was developed for the NG-CSSX solvent system based on the concepts used for the development of the BOBCalixC6 CSSX empirical equation for the estimation of $D_{\mathrm{Cs}}{ }^{10}$. The equation is able to predict $D_{\mathrm{Cs}}$ based of the initial concentrations of MaxCalix, $\mathrm{K}^{+}, \mathrm{OH}^{-}$, and $\mathrm{NO}_{3}{ }^{-}$.

$$
D_{C s}=[\bar{L}]_{i n i t} \cdot \frac{K_{1} \cdot\left[\mathrm{NO}_{3}^{-}\right]+K_{2} \cdot\left[\mathrm{OH}^{-}\right]}{1+\left[\mathrm{K}^{+}\right] \cdot\left(\mathrm{K}_{3}\left[\mathrm{NO}_{3}^{-}\right]+\mathrm{K}_{4}\left[\mathrm{OH}^{-}\right]\right)}
$$

The four constants of the equation, $\mathrm{K}_{1}, \mathrm{~K}_{2}, \mathrm{~K}_{3}, \& \mathrm{~K}_{4}$, were determined using the Microsoft Excel Solver function to determine the least sum of the squared differences between observed $D_{\mathrm{Cs}}$ values and ones calculated by Equation 7.2. Table 7.2 contains the systematic development of the four constants. The initial values were determined from the first ten samples and the subsequent values were determined by recalculation after including more samples in the order they were analyzed. This order is represented numerically in Table 7.3. To determine quality of fit, the calculated values were plotted against the observed values. A trendline with an intercept set to zero was added along with the $\mathrm{R}^{2}$ value. A perfect fit would yield a slope of one and an $\mathrm{R}^{2}$ value of one. As more samples were analyzed the constants were recalculated and the quality of fit was redetermined. 
The empirical equation predicts $D_{\mathrm{Cs}}$ within a $< \pm 10 \%$ error for simulant wastes; however, at high hydroxide concentrations the error increases as can be seen in Fig. 7.1. The results indicate that the empirical equation does not include important interactions that occur at high hydroxide concentrations. Further measurements of $D_{\mathrm{Cs}}$ at high concentrations of hydroxide would be necessary to improve the fit and better understand the important interactions.

Table 7.2. K values and quality of fit

\begin{tabular}{cccccc}
\hline $\begin{array}{c}\text { Number } \\
\text { of values }\end{array}$ & $\mathbf{R}^{2}$ value & $\begin{array}{c}\mathbf{K}_{\mathbf{1}} \\
\left(\mathbf{C s N O}_{\mathbf{3}}\right)\end{array}$ & $\begin{array}{c}\mathbf{K}_{\mathbf{2}} \\
(\mathbf{C s O H})\end{array}$ & $\begin{array}{c}\mathbf{K}_{\mathbf{3}} \\
\left(\mathbf{K N O}_{\mathbf{3}}\right)\end{array}$ & $\begin{array}{c}\mathbf{K}_{\mathbf{4}} \\
(\mathbf{K O H})\end{array}$ \\
\hline 10 & 0.9527 & 34.8 & 557.2 & 0.986 & 0.000 \\
20 & 0.9965 & 90.5 & 293.9 & 0.920 & 0.002 \\
30 & 0.9969 & 91.2 & 292.1 & 0.790 & 0.672 \\
40 & 0.9953 & 89.7 & 301.6 & 0.780 & 0.813 \\
50 & 0.9798 & 47.3 & 467.2 & 0.400 & 2.310 \\
60 & 0.9825 & 46.1 & 472.9 & 0.375 & 2.444 \\
70 & 0.9839 & 26.3 & 540.8 & 0.234 & 2.950 \\
74 & 0.9841 & 25.3 & 544.5 & 0.196 & 3.084 \\
\hline
\end{tabular}

Table 7.3. Sample numbers, compositions, experimental distribution ratios, and predicted distribution ratios

\begin{tabular}{|c|c|c|c|c|c|c|c|c|c|}
\hline Sample \# & [Max] & [Cs-7SB] & {$\left[\mathrm{Cs}^{+}\right]$} & {$\left[\mathrm{Na}^{+}\right]$} & {$\left[\mathbf{K}^{+}\right]$} & {$\left[\mathrm{OH}^{-}\right]$} & {$\left[\mathrm{NO}_{3}{ }^{-}\right]$} & Avg. $D_{C s}$ & $\begin{array}{c}\text { Pred. } \\
D_{C s}\end{array}$ \\
\hline 1 & 0.05001 & 0.49171 & 0.0001 & 5.60 & 0.00 & 1.00 & 4.6001 & 34.99 & 33.05 \\
\hline 2 & 0.05001 & 0.49171 & 0.0001 & 5.60 & 0.01 & 1.00 & 4.6101 & 36.03 & 31.80 \\
\hline 3 & 0.05001 & 0.49171 & 0.0001 & 5.60 & 0.02 & 1.00 & 4.6201 & 32.34 & 30.64 \\
\hline 4 & 0.05001 & 0.49171 & 0.0001 & 5.60 & 0.03 & 1.00 & 4.6301 & 31.35 & 29.55 \\
\hline 5 & 0.05001 & 0.49171 & 0.0001 & 5.60 & 0.04 & 1.00 & 4.6401 & 29.72 & 28.55 \\
\hline 6 & 0.05001 & 0.49171 & 0.0001 & 5.60 & 0.05 & 1.00 & 4.6501 & 29.72 & 27.61 \\
\hline 7 & 0.05001 & 0.49171 & 0.0001 & 5.60 & 0.06 & 1.00 & 4.6601 & 28.21 & 26.72 \\
\hline 8 & 0.05001 & 0.49171 & 0.0001 & 5.60 & 0.07 & 1.00 & 4.6701 & 27.95 & 25.90 \\
\hline 9 & 0.05001 & 0.49171 & 0.0001 & 5.60 & 0.08 & 1.00 & 4.6801 & 26.13 & 25.12 \\
\hline 10 & 0.05001 & 0.49171 & 0.0001 & 5.60 & 0.09 & 1.00 & 4.6901 & 24.98 & 24.39 \\
\hline 11 & 0.04943 & 0.49323 & 0.0001 & 5.60 & 0.10 & 1.00 & 4.7001 & 25.16 & 23.42 \\
\hline 12 & 0.04943 & 0.49323 & 0.0001 & 5.60 & 0.20 & 1.00 & 4.8001 & 19.78 & 18.24 \\
\hline 13 & 0.04943 & 0.49323 & 0.0001 & 5.60 & 0.30 & 1.00 & 4.9001 & 15.94 & 14.94 \\
\hline 14 & 0.04943 & 0.49323 & 0.0001 & 5.60 & 0.40 & 1.00 & 5.0001 & 13.07 & 12.64 \\
\hline 15 & 0.04943 & 0.49323 & 0.0001 & 5.60 & 0.50 & 1.00 & 5.1001 & 11.20 & 10.95 \\
\hline 16 & 0.04943 & 0.49323 & 0.0001 & 5.60 & 0.60 & 1.00 & 5.2001 & 9.61 & 9.66 \\
\hline 17 & 0.04943 & 0.49323 & 0.0001 & 5.60 & 0.70 & 1.00 & 5.3001 & 8.53 & 8.64 \\
\hline 18 & 0.04943 & 0.49323 & 0.0001 & 5.60 & 0.80 & 1.00 & 5.4001 & 7.79 & 7.81 \\
\hline 19 & 0.04943 & 0.49323 & 0.0001 & 5.60 & 0.90 & 1.00 & 5.5001 & 6.98 & 7.12 \\
\hline 20 & 0.04943 & 0.49323 & 0.0001 & 5.60 & 1.00 & 1.00 & 5.6001 & 6.27 & 6.55 \\
\hline 21 & 0.04943 & 0.49323 & 0.0001 & 5.60 & 0.00 & 1.50 & 4.1001 & 40.62 & 45.50 \\
\hline 22 & 0.04943 & 0.49323 & 0.0001 & 5.60 & 0.01 & 1.50 & 4.1101 & 37.86 & 43.17 \\
\hline 23 & 0.04943 & 0.49323 & 0.0001 & 5.60 & 0.02 & 1.50 & 4.1201 & 36.56 & 41.07 \\
\hline 24 & 0.04943 & 0.49323 & 0.0001 & 5.60 & 0.03 & 1.50 & 4.1301 & 36.42 & 39.16 \\
\hline
\end{tabular}


Table 7.3. Sample numbers, compositions, experimental distribution ratios, and predicted distribution ratios

\begin{tabular}{|c|c|c|c|c|c|c|c|c|c|}
\hline Sample \# & [Max] & [Cs-7SB] & {$\left[\mathrm{Cs}^{+}\right]$} & {$\left[\mathrm{Na}^{+}\right]$} & {$\left[\mathbf{K}^{+}\right]$} & {$\left[\mathrm{OH}^{-}\right]$} & {$\left[\mathrm{NO}_{3}{ }^{-}\right]$} & Avg. $D_{C s}$ & $\begin{array}{c}\text { Pred. } \\
D_{C s}\end{array}$ \\
\hline 25 & 0.04943 & 0.49323 & 0.0001 & 5.60 & 0.04 & 1.50 & 4.1401 & 34.40 & 37.42 \\
\hline 26 & 0.04943 & 0.49323 & 0.0001 & 5.60 & 0.05 & 1.50 & 4.1501 & 33.53 & 35.82 \\
\hline 27 & 0.04943 & 0.49323 & 0.0001 & 5.60 & 0.06 & 1.50 & 4.1601 & 32.20 & 34.36 \\
\hline 28 & 0.04943 & 0.49323 & 0.0001 & 5.60 & 0.07 & 1.50 & 4.1701 & 30.49 & 33.01 \\
\hline 29 & 0.04943 & 0.49323 & 0.0001 & 5.60 & 0.08 & 1.50 & 4.1801 & 30.98 & 31.77 \\
\hline 30 & 0.04943 & 0.49323 & 0.0001 & 5.60 & 0.09 & 1.50 & 4.1901 & 28.58 & 30.61 \\
\hline 31 & 0.04919 & 0.49443 & 0.0001 & 5.60 & 0.10 & 1.50 & 4.2001 & 30.97 & 29.39 \\
\hline 32 & 0.04919 & 0.49443 & 0.0001 & 5.60 & 0.20 & 1.50 & 4.3001 & 22.20 & 21.75 \\
\hline 33 & 0.04919 & 0.49443 & 0.0001 & 5.60 & 0.30 & 1.50 & 4.4001 & 17.73 & 17.26 \\
\hline 34 & 0.04919 & 0.49443 & 0.0001 & 5.60 & 0.40 & 1.50 & 4.5001 & 13.80 & 14.30 \\
\hline 35 & 0.04919 & 0.49443 & 0.0001 & 5.60 & 0.50 & 1.50 & 4.6001 & 11.54 & 12.20 \\
\hline 36 & 0.04919 & 0.49443 & 0.0001 & 5.60 & 0.60 & 1.50 & 4.7001 & 10.29 & 10.64 \\
\hline 37 & 0.04919 & 0.49443 & 0.0001 & 5.60 & 0.70 & 1.50 & 4.8001 & 9.01 & 9.43 \\
\hline 38 & 0.04919 & 0.49443 & 0.0001 & 5.60 & 0.80 & 1.50 & 4.9001 & 7.87 & 8.46 \\
\hline 39 & 0.04919 & 0.49443 & 0.0001 & 5.60 & 0.90 & 1.50 & 5.0001 & 7.34 & 7.68 \\
\hline 40 & 0.04919 & 0.49443 & 0.0001 & 5.60 & 1.00 & 1.50 & 5.1001 & 6.45 & 7.02 \\
\hline 41 & 0.04901 & 0.49498 & 0.0001 & 5.60 & 0.00 & 2.00 & 3.6001 & 52.31 & 57.84 \\
\hline 42 & 0.04901 & 0.49498 & 0.0001 & 5.60 & 0.01 & 2.00 & 3.6101 & 55.90 & 54.13 \\
\hline 43 & 0.04901 & 0.49498 & 0.0001 & 5.60 & 0.02 & 2.00 & 3.6201 & 55.34 & 50.87 \\
\hline 44 & 0.04901 & 0.49498 & 0.0001 & 5.60 & 0.03 & 2.00 & 3.6301 & 46.20 & 47.98 \\
\hline 45 & 0.04901 & 0.49498 & 0.0001 & 5.60 & 0.04 & 2.00 & 3.6401 & 42.77 & 45.40 \\
\hline 46 & 0.04901 & 0.49498 & 0.0001 & 5.60 & 0.05 & 2.00 & 3.6501 & 41.66 & 43.08 \\
\hline 47 & 0.04901 & 0.49498 & 0.0001 & 5.60 & 0.06 & 2.00 & 3.6601 & 38.87 & 40.99 \\
\hline 48 & 0.04901 & 0.49498 & 0.0001 & 5.60 & 0.07 & 2.00 & 3.6701 & 42.04 & 39.09 \\
\hline 49 & 0.04901 & 0.49498 & 0.0001 & 5.60 & 0.08 & 2.00 & 3.6801 & 36.42 & 37.36 \\
\hline 50 & 0.04901 & 0.49498 & 0.0001 & 5.60 & 0.09 & 2.00 & 3.6901 & 34.20 & 35.77 \\
\hline 51 & 0.04981 & 0.5000 & 0.0001 & 5.60 & 0.10 & 2.00 & 3.7001 & 36.22 & 34.87 \\
\hline 52 & 0.04981 & 0.5000 & 0.0001 & 5.60 & 0.20 & 2.00 & 3.8001 & 25.54 & 24.78 \\
\hline 53 & 0.04981 & 0.5000 & 0.0001 & 5.60 & 0.30 & 2.00 & 3.9001 & 19.36 & 19.21 \\
\hline 54 & 0.04981 & 0.5000 & 0.0001 & 5.60 & 0.40 & 2.00 & 4.0001 & 14.85 & 15.68 \\
\hline 55 & 0.04981 & 0.5000 & 0.0001 & 5.60 & 0.50 & 2.00 & 4.1001 & 12.72 & 13.25 \\
\hline 56 & 0.04981 & 0.5000 & 0.0001 & 5.60 & 0.60 & 2.00 & 4.2001 & 10.88 & 11.46 \\
\hline 57 & 0.04981 & 0.5000 & 0.0001 & 5.60 & 0.70 & 2.00 & 4.3001 & 9.21 & 10.10 \\
\hline 58 & 0.04981 & 0.5000 & 0.0001 & 5.60 & 0.80 & 2.00 & 4.4001 & 8.20 & 9.03 \\
\hline 59 & 0.04981 & 0.5000 & 0.0001 & 5.60 & 0.90 & 2.00 & 4.5001 & 7.49 & 8.16 \\
\hline 60 & 0.04981 & 0.5000 & 0.0001 & 5.60 & 1.00 & 2.00 & 4.6001 & 6.70 & 7.44 \\
\hline 61 & 0.04981 & 0.5000 & 0.0001 & 5.60 & 0.00 & 2.50 & 3.1001 & 76.10 & 71.71 \\
\hline 62 & 0.04981 & 0.5000 & 0.0001 & 5.60 & 0.01 & 2.50 & 3.1101 & 70.79 & 66.21 \\
\hline 63 & 0.04981 & 0.5000 & 0.0001 & 5.60 & 0.02 & 2.50 & 3.1201 & 58.15 & 61.50 \\
\hline 64 & 0.04981 & 0.5000 & 0.0001 & 5.60 & 0.03 & 2.50 & 3.1301 & 58.56 & 57.41 \\
\hline 65 & 0.04981 & 0.5000 & 0.0001 & 5.60 & 0.04 & 2.50 & 3.1401 & 54.20 & 53.83 \\
\hline 66 & 0.04981 & 0.5000 & 0.0001 & 5.60 & 0.05 & 2.50 & 3.1501 & 53.73 & 50.68 \\
\hline 67 & 0.04981 & 0.5000 & 0.0001 & 5.60 & 0.07 & 2.50 & 3.1701 & 47.16 & 45.35 \\
\hline 68 & 0.04981 & 0.5000 & 0.0001 & 5.60 & 0.08 & 2.50 & 3.1801 & 45.63 & 43.09 \\
\hline 69 & 0.04981 & 0.5000 & 0.0001 & 5.60 & 0.09 & 2.50 & 3.1901 & 42.12 & 41.04 \\
\hline 70 & 0.04981 & 0.5000 & 0.0001 & 5.60 & 0.10 & 2.50 & 3.2001 & 39.65 & 39.18 \\
\hline 71 & 0.04981 & 0.5000 & 0.0001 & 5.60 & 0.20 & 2.50 & 3.3001 & 26.61 & 26.94 \\
\hline 72 & 0.04981 & 0.5000 & 0.0001 & 5.60 & 0.30 & 2.50 & 3.4001 & 19.60 & 20.52 \\
\hline 73 & 0.04981 & 0.5000 & 0.0001 & 5.60 & 0.40 & 2.50 & 3.5001 & 15.66 & 16.57 \\
\hline 74 & 0.04981 & 0.5000 & 0.0001 & 5.60 & 0.50 & 2.50 & 3.6001 & 12.77 & 13.89 \\
\hline
\end{tabular}


Table 7.4. Savannah River Site simulant $\boldsymbol{D}_{\mathrm{Cs}}$

\begin{tabular}{lllllllll}
\hline & {$[\mathbf{M a x}]$} & {$[\mathbf{C s - 7 S B}]$} & {$\left[\mathbf{C s}^{+}\right]$} & {$\left[\mathbf{N a}^{+}\right]$} & {$\left[\mathbf{K}^{+}\right]$} & {$\left[\mathbf{O H}^{-}\right]$} & {$\left[\mathbf{N O}_{3}{ }^{-}\right]$} & Avg. $\boldsymbol{D}_{C s}$ \\
\hline Q/A & 0.04901 & 0.4950 & $1.00 \times 10^{-4}$ & 5.00 & 0.00 & 2.00 & 3.00 & 47.83 \\
SRS-15 & 0.04901 & 0.4950 & $1.40 \times 10^{-4}$ & 5.60 & 0.015 & 2.06 & 2.03 & 55.00 \\
\hline SRS-45 & 0.04901 & 0.4950 & $1.40 \times 10^{-4}$ & 5.60 & 0.045 & 2.06 & 2.06 & 44.07 \\
\hline
\end{tabular}

Table 7.5. Prediction quality for SRS-15 simulant

\begin{tabular}{cccccccr}
\hline $\begin{array}{c}\text { Number } \\
\text { of values }\end{array}$ & $\mathbf{R}^{\mathbf{2}}$ value & $\begin{array}{c}\mathbf{K}_{\mathbf{1}} \\
(\mathbf{C s N O})\end{array}$ & $\begin{array}{c}\mathbf{K}_{\mathbf{2}} \\
(\mathbf{C s O H})\end{array}$ & $\begin{array}{c}\mathbf{K}_{\mathbf{3}} \\
\left(\mathbf{K N O}_{\mathbf{3}}\right)\end{array}$ & $\begin{array}{c}\mathbf{K}_{\mathbf{4}} \\
(\mathbf{K O H})\end{array}$ & $\begin{array}{c}\text { Pred. SRS- } \\
\mathbf{1 5}\end{array}$ & $\mathbf{\%} \mathbf{E r r}$ \\
\hline 10 & 0.9527 & 34.8 & 557.2 & 0.986 & 0.000 & 57.98 & 5.41 \\
20 & 0.9965 & 90.5 & 293.9 & 0.920 & 0.002 & 37.62 & 31.60 \\
30 & 0.9969 & 91.2 & 292.1 & 0.790 & 0.672 & 36.91 & 32.83 \\
40 & 0.9953 & 89.7 & 301.6 & 0.780 & 0.813 & 37.54 & 31.75 \\
50 & 0.9798 & 47.3 & 467.2 & 0.400 & 2.310 & 47.87 & 12.96 \\
60 & 0.9825 & 46.1 & 472.9 & 0.375 & 2.444 & 48.15 & 12.46 \\
70 & 0.9839 & 26.3 & 540.8 & 0.234 & 2.950 & 52.10 & 5.28 \\
\hline 74 & 0.9841 & 25.3 & 544.5 & 0.196 & 3.084 & 52.21 & 5.03 \\
\hline
\end{tabular}

Table 7.6. Prediction quality for SRS-45 simulant

\begin{tabular}{|c|c|c|c|c|c|c|c|}
\hline $\begin{array}{l}\text { Number } \\
\text { of values }\end{array}$ & $R^{2}$ value & $\begin{array}{c}\mathrm{K}_{1} \\
\left(\mathrm{CsNO}_{3}\right)\end{array}$ & $\begin{array}{c}\mathrm{K}_{2} \\
(\mathrm{CsOH})\end{array}$ & $\begin{array}{c}\mathrm{K}_{3} \\
\left(\mathrm{KNO}_{3}\right)\end{array}$ & $\begin{array}{c}\mathrm{K}_{4} \\
(\mathrm{KOH})\end{array}$ & $\begin{array}{c}\text { Pred. SRS- } \\
45\end{array}$ & $\%$ Err \\
\hline 10 & 0.9527 & 34.8 & 557.2 & 0.986 & 0.000 & 54.76 & 24.27 \\
\hline 20 & 0.9965 & 90.5 & 293.9 & 0.920 & 0.002 & 35.75 & 18.86 \\
\hline 30 & 0.9969 & 91.2 & 292.1 & 0.790 & 0.672 & 34.08 & 22.67 \\
\hline 40 & 0.9953 & 89.7 & 301.6 & 0.780 & 0.813 & 34.42 & 21.89 \\
\hline 50 & 0.9798 & 47.3 & 467.2 & 0.400 & 2.310 & 41.51 & 5.79 \\
\hline 60 & 0.9825 & 46.1 & 472.9 & 0.375 & 2.444 & 41.54 & 5.73 \\
\hline 70 & 0.9839 & 26.3 & 540.8 & 0.234 & 2.950 & 44.21 & 0.32 \\
\hline 74 & 0.9841 & 25.3 & 544.5 & 0.196 & 3.084 & 44.12 & 0.11 \\
\hline
\end{tabular}

\section{Calculated vs. Observed Distributions}

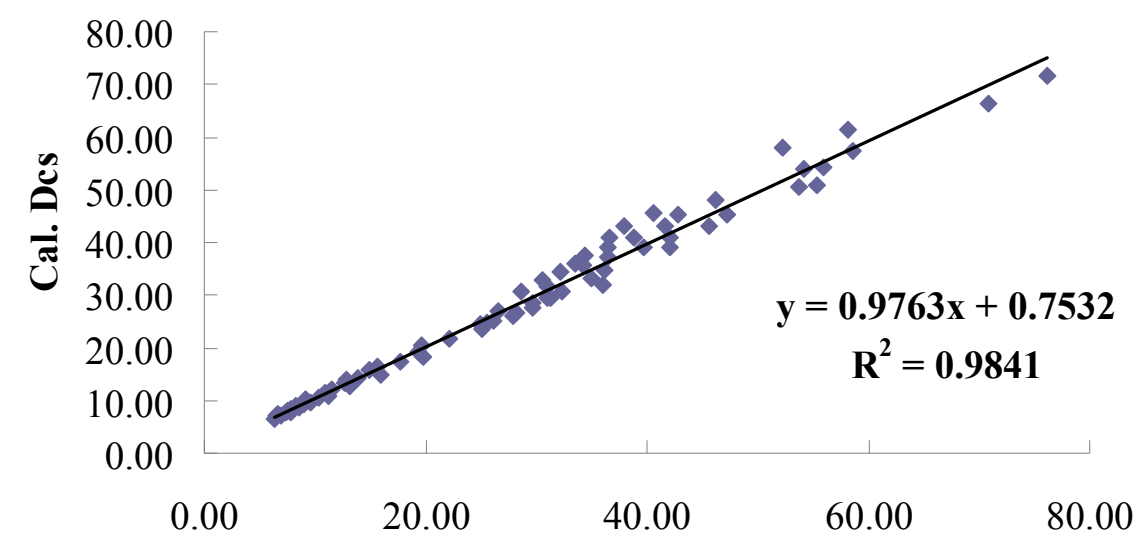

Obs. Des

Fig. 7.1. Correlation between $D_{\mathrm{Cs}}$ obtained experimentally and the $D_{\mathrm{Cs}}$ calculated using eq. 7.2. 
$D_{\text {Cs }}$ measured with the NG-CSSX solvent system using simulated tank wastes SRS-15 and SRS-45 are shown in Table 7.3. Tables 7.4 and 7.5 show the quality of predicted $D_{\mathrm{Cs}}$ values for simulants as the constants were developed. The best predictions result from using the full data set, yielding predicted $D_{\mathrm{Cs}}$ values for both simulants with $<10 \%$ error. The percent error of predicted $D_{\mathrm{Cs}}$ will be at greatest at $\left[\mathrm{OH}^{-}\right]$ larger than $2.5 \mathrm{M}$. The empirical equation can be used to predict $D_{\mathrm{Cs}}$ values for waste stream using only inputs of $[\mathrm{MaxCalix}],\left[\mathrm{OH}^{-}\right],\left[\mathrm{K}^{+}\right]$, and $\left[\mathrm{NO}_{3}{ }^{-}\right]$. Equation 7.3 shows the equation with optimized constants. The equation will fail when applied to systems that do not contain one of these key species.

$$
D_{\mathrm{Cs}}=[\bar{L}]_{\text {init }} \cdot \frac{25.3 \cdot\left[\mathrm{NO}_{3}^{-}\right]+544.5 \cdot\left[\mathrm{OH}^{-}\right]}{1+\left[\mathrm{K}^{+}\right] \cdot\left(.196\left[\mathrm{NO}_{3}^{-}\right]+3.084\left[\mathrm{OH}^{-}\right]\right)}
$$

\subsection{CONCLUSIONS}

An empirical equation for the estimation of $D_{\mathrm{Cs}}$ for the NG-CSSX solvent system was successfully derived; based on the concepts used for the empirical equation used to estimate $D_{\mathrm{Cs}}$ values for the original

BOBCalixC6 CSSX solvent ${ }^{10}$. The equation is able to predict $D_{\mathrm{Cs}}$ based of the initial concentrations of MaxCalix, $\mathrm{K}^{+}, \mathrm{OH}^{-}$, and $\mathrm{NO}_{3}^{-}$. The empirical equation predicts $D_{\mathrm{Cs}}$ within a $< \pm 10 \%$ error for simulant wastes, however at high hydroxide concentrations the error increases significantly. One flaw of the formula however, is that all four principle components, $\mathrm{K}^{+}, \mathrm{OH}^{-}$, and $\mathrm{NO}_{3}{ }^{-}$in the simulant and MaxCalix in the solvent, must be present for the accurate prediction of $D_{\mathrm{Cs}}$ values. 



\section{EXTRACTION BEHAVIOR OF FINAL SOLVENT UNDER VARYING CONDITIONS}

This work was undertaken to quantify the effect of temperature, alkaline metal concentration and other possible physical and chemical stresses on the extraction, scrubbing, and stripping in the NG-CSSX process. A strong temperature dependence of the $D_{C s}$ in the baseline BOBCalixC6 CSSX process necessitated temperature control in the MCU at the SRS. It was therefore considered likely that a similar temperature dependence would be encountered for NG-CSSX, but given the different solvent composition and new aqueous scrub and strip phases of NG-CSSX, the magnitude of the effect was not predictable. ESS batch tests were performed with average SRS salt feed simulant (SRS-15) at 15,25 , and $35^{\circ} \mathrm{C}$. A much steeper temperature dependence was found for NG-CSSX in comparison with the currently employed BOBCalixC6 CSSX process in the MCU. Whereas $D_{\mathrm{Cs}}$ decreases 3.7-fold for the extraction step of BOBCalixC6 CSSX when the temperature is increased from $15{ }^{\circ} \mathrm{C}$ to $35^{\circ} \mathrm{C}, D_{\mathrm{Cs}}$ decreases 11.5 -fold for the extraction step of NG-CSSX over the same temperature range. Scrubbing and stripping in NG-CSSX also show an order-of-magnitude drop in $D_{\mathrm{Cs}}$. As before, it may be concluded that NG-CSSX process performance and predictability depends on the implementation of adequate temperature control and may be enhanced with lower temperature during extraction and higher temperature in stripping.

A batch ESS experiment using simulated SRS waste compositions demonstrated that the cesium extraction strength of the NG-CSSX solvent increases with waste concentration above the design feed concentration of $5.6 \mathrm{M}$ sodium. A series of five SRS simulants were utilized in the experiment with the concentrations of sodium ranging from $3.5 \mathrm{M}$ to $8 \mathrm{M}$, all components remaining in the same proportion. Assuming that hydraulics and stage efficiency are not negatively affected, the simple batch results obtained here would imply that the NG-CSSX process could support an increased waste-processing rate based on increased waste concentration while maintaining required waste decontamination. Since potassium concentration could potentially be a limiting factor for the efficacy of the solvent in the removal of cesium ${ }^{2}$ further simulants were analyzed (SRS-45 and Hanford) and compared with SRS-15. The batch tests confirm that, although the cesium decontamination is affected by increased feed potassium concentration, the NG-CSSX solvent has the potential to be applied to feeds with higher potassium levels. 


\subsection{MATERIALS}

\subsubsection{Waste Simulant Compositions}

The targeted composition of the SRS-15 tank-waste simulant used in the majority of the extraction studies is highlighted in Table 8.1 according to the preparative method described in detail in Chapter 2. Appropriate amounts of reagent-grade chemicals were weighed into volumetric flasks in the order prescribed. Significant precipitate formed upon adding the $\mathrm{NaOH}$, which is considered normal, and the mixture was stirred for several hours and then allowed to settle at least for a few days before use. The SRS-15 simulant is designed to represent the average SRS tank-waste composition ${ }^{2}$. Table 8.2 details the two simulants SRS-45 and Hanford, both containing significantly larger concentrations of potassium. Constituent species are named in the tables as the chemical forms added, not necessarily to be taken as the forms actually present after mixing. Appropriate amounts of reagent-grade chemicals were weighed into volumetric flasks. Actual component concentrations therefore may differ from the concentrations tabulated ${ }^{1}$. Tracer ${ }^{137} \mathrm{Cs}$ was added to the simulant only for "hot" ESS tests $(4 \mu \mathrm{L}$ spike from a $0.05 \mathrm{mCi} / \mathrm{mL}$ stock, $\mathrm{CsCl}$ in $\mathrm{H}_{2} \mathrm{O}$, Eckert \& Ziegler Isotope Products Inc., formally Isotope Products, Burbank, CA). Scrub solution $(0.025 \mathrm{M} \mathrm{NaOH})$ and other $\mathrm{NaOH}$ solutions were prepared by dilution of $1.0 \mathrm{M}$ $\mathrm{NaOH}$ standard solution (VWR). Strip solution $\left(0.010 \mathrm{M} \mathrm{H}_{3} \mathrm{BO}_{3}\right)$ was obtained by dilution of a $0.1 \mathrm{M} \mathrm{H}_{3} \mathrm{BO}_{3}$ stock solution prepared from lab-grade $\mathrm{H}_{3} \mathrm{BO}_{3}$ (min 95.5\%, Sigma Aldrich). Solutions of $\mathrm{HCl}$ were made from 1.0 M HCl standard solution (Baker). Water for preparation of all aqueous solutions was first distilled and then deionized using a Milli- $\mathrm{Q}^{\circledR}$ gradient A10 filtering system equipped with a Quantum ${ }^{\mathrm{TM}}$ Ex Ultrapure Organex Cartridge $\left(18.2 \mathrm{M} \Omega \cdot \mathrm{cm}\right.$ at $25^{\circ} \mathrm{C}$, total organic content $4 \mathrm{ppb}$ ).

Table 8.1. Target composition of aqueous tank-waste simulants - Part I

\begin{tabular}{|c|c|c|c|c|c|}
\hline Analyte or Species & $\begin{array}{l}\text { SRS-9 } \\
\mathrm{mol} / \mathrm{L}\end{array}$ & $\begin{array}{c}\text { SRS-12 } \\
\mathrm{mol} / \mathrm{L}\end{array}$ & $\begin{array}{c}\text { SRS-15 } \\
\mathrm{mol} / \mathrm{L}\end{array}$ & $\begin{array}{c}\text { SRS-19 } \\
\mathrm{mol} / \mathrm{L}\end{array}$ & $\begin{array}{c}\text { SRS-22 } \\
\mathrm{mol} / \mathrm{L}\end{array}$ \\
\hline \multicolumn{6}{|l|}{ Principal constituents: } \\
\hline $\mathrm{Al}$ as $\left(\mathrm{Al}(\mathrm{OH})_{4}\right)^{-}$ & 0.175 & 0.255 & 0.280 & 0.340 & 0.400 \\
\hline $\mathrm{Cl}^{-}$ & $1.5 \times 10^{-2}$ & $1.9 \times 10^{-2}$ & $2.4 \times 10^{-} 2$ & $2.9 \times 10^{-2}$ & $3.4 \times 10^{-2}$ \\
\hline $\mathrm{CO}_{3}{ }^{2-}$ (TIC) & 0.075 & 0.120 & 0.150 & 0.182 & 0.214 \\
\hline $\mathrm{Cs}^{+}$(Total) & $8.8 \times 10^{-5}$ & $1.1 \times 10^{-4}$ & $1.4 \times 10^{-} 4$ & $1.7 \times 10^{-4}$ & $2.0 \times 10^{-4}$ \\
\hline $\mathrm{K}^{+}$ & 0.009 & 0.012 & 0.015 & 0.019 & 0.022 \\
\hline $\mathrm{Na}^{+}$ & 3.50 & 4.50 & 5.60 & 6.80 & 8.00 \\
\hline $\mathrm{NO}_{2}^{-}$ & 0.312 & 0.402 & 0.500 & 0.608 & 0.715 \\
\hline $\mathrm{NO}_{3}^{-}$ & 1.27 & 1.63 & 2.03 & 2.50 & 2.90 \\
\hline $\mathrm{OH}^{-}$(Free) & 1.29 & 1.66 & 2.06 & 2.50 & 2.94 \\
\hline $\mathrm{SO}_{4}^{2-}$ & 0.088 & 0.112 & 0.140 & 0.170 & 0.200 \\
\hline
\end{tabular}

Minor inorganic constituents: 
Table 8.1. Target composition of aqueous tank-waste simulants - Part I

\begin{tabular}{|c|c|c|c|c|c|}
\hline Analyte or Species & $\begin{array}{l}\text { SRS-9 } \\
\mathrm{mol} / \mathrm{L}\end{array}$ & $\begin{array}{c}\text { SRS-12 } \\
\mathrm{mol} / \mathrm{L}\end{array}$ & $\begin{array}{c}\text { SRS-15 } \\
\mathrm{mol} / \mathrm{L}\end{array}$ & $\begin{array}{c}\text { SRS-19 } \\
\mathrm{mol} / \mathrm{L}\end{array}$ & $\begin{array}{c}\text { SRS-22 } \\
\mathrm{mol} / \mathrm{L}\end{array}$ \\
\hline $\operatorname{Ag}(\mathrm{I})$ & $5.8 \times 10^{-8}$ & $7.5 \times 10^{-8}$ & $9.3 \times 10^{-8}$ & $1.1 \times 10^{-7}$ & $1.3 \times 10^{-7}$ \\
\hline $\mathrm{CrO}_{4}^{2-}$ & $8.8 \times 10^{-4}$ & $1.1 \times 10^{-3}$ & $1.4 \times 10^{-3}$ & $1.7 \times 10^{-3}$ & $2.0 \times 10^{-3}$ \\
\hline $\mathrm{Cu}(\mathrm{II})$ & $1.4 \times 10^{-5}$ & $1.8 \times 10^{-5}$ & $2.3 \times 10^{-5}$ & $2.8 \times 10^{-5}$ & $3.3 \times 10^{-5}$ \\
\hline $\mathrm{F}^{-}$ & $1.8 \times 10^{-2}$ & $2.2 \times 10^{-2}$ & $2.8 \times 10^{-2}$ & $3.4 \times 10^{-2}$ & $4.0 \times 10^{-2}$ \\
\hline $\mathrm{Fe}(\mathrm{III})$ & $1.6 \times 10^{-5}$ & $2.1 \times 10^{-5}$ & $2.6 \times 10^{-5}$ & $3.2 \times 10^{-5}$ & $3.7 \times 10^{-5}$ \\
\hline $\mathrm{Hg}(\mathrm{II})$ & $1.6 \times 10^{-7}$ & $2.0 \times 10^{-7}$ & $2.5 \times 10^{-7}$ & $3.0 \times 10^{-7}$ & $3.5 \times 10^{-7}$ \\
\hline $\mathrm{MoO}_{4}^{2-}$ & $4.4 \times 10^{-5}$ & $5.6 \times 10^{-5}$ & $7.0 \times 10^{-5}$ & $8.5 \times 10^{-5}$ & $1.0 \times 10^{-4}$ \\
\hline $\mathrm{NH}_{3}$ & $6.2 \times 10^{-4}$ & $8.0 \times 10^{-4}$ & $1.0 \times 10^{-3}$ & $1.2 \times 10^{-3}$ & $1.4 \times 10^{-3}$ \\
\hline $\mathrm{Pb}(\mathrm{II})$ & $6.2 \times 10^{-6}$ & $8.0 \times 10^{-6}$ & $1.0 \times 10^{-5}$ & $1.2 \times 10^{-5}$ & $1.4 \times 10^{-5}$ \\
\hline $\operatorname{Pd}(\mathrm{II})$ & $2.4 \times 10^{-6}$ & $3.0 \times 10^{-6}$ & $3.8 \times 10^{-6}$ & $4.6 \times 10^{-6}$ & $5.4 \times 10^{-6}$ \\
\hline $\mathrm{PO}_{4}^{3-}$ & $4.4 \times 10^{-3}$ & $5.6 \times 10^{-3}$ & $7.0 \times 10^{-3}$ & $8.5 \times 10^{-3}$ & $1.0 \times 10^{-2}$ \\
\hline $\mathrm{Rh}(\mathrm{III})$ & $1.2 \times 10^{-6}$ & $1.6 \times 10^{-6}$ & $2.0 \times 10^{-6}$ & $2.4 \times 10^{-6}$ & $2.8 \times 10^{-6}$ \\
\hline $\mathrm{Ru}(\mathrm{III})$ & $5.1 \times 10^{-6}$ & $6.5 \times 10^{-6}$ & $8.1 \times 10^{-6}$ & $9.8 \times 10^{-6}$ & $1.2 \times 10^{-5}$ \\
\hline $\mathrm{Si}(\mathrm{IV})$ & $1.9 \times 10^{-2}$ & $2.4 \times 10^{-2}$ & $3.0 \times 10^{-2}$ & $3.6 \times 10^{-2}$ & $4.3 \times 10^{-2}$ \\
\hline $\mathrm{Sn}(\mathrm{II})$ & $1.2 \times 10^{-5}$ & $1.6 \times 10^{-5}$ & $2.0 \times 10^{-5}$ & $2.4 \times 10^{-5}$ & $2.8 \times 10^{-5}$ \\
\hline $\begin{array}{l}\text { Minor organic constituents: } \\
n \text {-Butanol }\end{array}$ & $1.7 \times 10^{-5}$ & $2.2 \times 10^{-5}$ & $2.7 \times 10^{-5}$ & $3.3 \times 10^{-5}$ & $3.8 \times 10^{-5}$ \\
\hline Tri-n-butylphosphate (TBP) & $1.2 \times 10^{-6}$ & $1.5 \times 10^{-6}$ & $1.9 \times 10^{-6}$ & $2.3 \times 10^{-6}$ & $2.7 \times 10^{-6}$ \\
\hline Di-n-butylphosphate (DBP) & $7.5 \times 10^{-5}$ & $9.6 \times 10^{-5}$ & $1.2 \times 10^{-4}$ & $1.4 \times 10^{-4}$ & $1.7 \times 10^{-4}$ \\
\hline $\begin{array}{l}\text { Mono- } n \text {-butylphosphate } \\
\text { (MBP) }\end{array}$ & $1.0 \times 10^{-4}$ & $1.3 \times 10^{-4}$ & $1.6 \times 10^{-4}$ & $1.9 \times 10^{-4}$ & $2.3 \times 10^{-4}$ \\
\hline Formate $\left(\mathrm{HCO}_{2}^{-}\right)$ & $2.1 \times 10^{-2}$ & $2.6 \times 10^{-2}$ & $3.3 \times 10^{-2}$ & $4.0 \times 10^{-2}$ & $4.7 \times 10^{-2}$ \\
\hline Oxalate $\left(\mathrm{C}_{2} \mathrm{O}_{4}{ }^{2-}\right)$ & $5.0 \times 10^{-3}$ & $6.4 \times 10^{-3}$ & $8.0 \times 10^{-3}$ & $9.7 \times 10^{-3}$ & $1.1 \times 10^{-4}$ \\
\hline Trimethylamine & $1.1 \times 10^{-4}$ & $1.4 \times 10^{-4}$ & $1.7 \times 10^{-4}$ & $2.1 \times 10^{-4}$ & $2.4 \times 10^{-4}$ \\
\hline
\end{tabular}

Table 8.2. Target composition of aqueous tank-waste simulants - Part II

\begin{tabular}{|c|c|c|c|c|c|}
\hline Analyte or Species & $\begin{array}{c}\text { SRS-45 } \\
\mathrm{mol} / \mathrm{L}\end{array}$ & $\begin{array}{c}\text { Hanford } \\
\mathrm{mol} / \mathrm{L}\end{array}$ & $\begin{array}{c}\text { Cs-Free } \\
\text { mol/L }\end{array}$ & $\begin{array}{c}\text { SRS-SDS } \\
\mathrm{mol} / \mathrm{L}\end{array}$ & $\begin{array}{c}\text { SDS-Spike } \\
\text { mol/L }\end{array}$ \\
\hline \multicolumn{6}{|l|}{ Principal constituents: } \\
\hline $\mathrm{Al}$ as $\left(\mathrm{Al}(\mathrm{OH})_{4}\right)$ & 0.280 & 0.570 & 0.280 & 0.280 & 0.280 \\
\hline $\mathrm{Cl}^{-}$ & $2.4 \times 10^{-2}$ & $9.4 \times 10^{-2}$ & $2.4 \times 10^{-2}$ & $2.4 \times 10^{-2}$ & $2.4 \times 10^{-2}$ \\
\hline $\mathrm{CO}_{3}{ }^{2-}$ (TIC) & 0.150 & 0.367 & 0.150 & 0.150 & 0.150 \\
\hline $\mathrm{Cs}^{+}$(Total) & $1.4 \times 10^{-4}$ & $5.82 \times 10^{-5}$ & - & $1.4 \times 10^{-4}$ & $1.4 \times 10^{-4}$ \\
\hline $\mathbf{K}^{+}$ & 0.045 & 0.174 & 0.015 & 0.015 & - \\
\hline $\mathrm{Na}^{+}$ & 5.60 & 6.00 & 5.60 & 5.60 & 5.60 \\
\hline $\mathrm{NO}_{2}^{-}$ & 0.500 & 1.17 & 0.500 & 0.500 & 0.500 \\
\hline $\mathrm{NO}_{3}^{-}$ & 2.06 & 1.90 & 2.03 & 2.03 & 2.03 \\
\hline $\mathrm{OH}^{-}$(Free) & 2.06 & 1.45 & 2.06 & 2.06 & 2.06 \\
\hline $\mathrm{SO}_{4}{ }^{2-}$ & 0.140 & $3.22 \times 10^{-2}$ & 0.140 & 0.140 & - \\
\hline \multicolumn{6}{|l|}{$\begin{array}{l}\text { Minor inorganic } \\
\text { constituents: }\end{array}$} \\
\hline $\operatorname{Ag}(\mathrm{I})$ & $9.3 \times 10^{-8}$ & - & $9.3 \times 10^{-8}$ & $9.3 \times 10^{-8}$ & - \\
\hline Bi(III) & - & $5.6 \times 10^{-5}$ & $1.4 \times 10^{-3}$ & $1.4 \times 10^{-3}$ & - \\
\hline $\mathrm{Ca}^{2+}$ & - & $3.8 \times 10^{-4}$ & $2.3 \times 10^{-5}$ & $2.3 \times 10^{-5}$ & - \\
\hline $\mathrm{CrO}_{4}{ }^{2-}$ & $1.4 \times 10^{-3}$ & $1.0 \times 10^{-2}$ & $2.8 \times 10^{-2}$ & $2.8 \times 10^{-2}$ & - \\
\hline $\mathrm{Cu}(\mathrm{II})$ & $2.3 \times 10^{-5}$ & - & $2.6 \times 10^{-5}$ & $2.6 \times 10^{-5}$ & - \\
\hline $\mathrm{F}^{-}$ & $2.8 \times 10^{-2}$ & $1.3 \times 10^{-2}$ & $2.5 \times 10^{-7}$ & $2.5 \times 10^{-7}$ & - \\
\hline $\mathrm{Fe}(\mathrm{III})$ & $2.6 \times 10^{-5}$ & $1.0 \times 10^{-4}$ & $7.0 \times 10^{-5}$ & $7.0 \times 10^{-5}$ & - \\
\hline
\end{tabular}


Table 8.2. Target composition of aqueous tank-waste simulants - Part II

\begin{tabular}{|c|c|c|c|c|c|}
\hline Analyte or Species & $\begin{array}{l}\text { SRS-45 } \\
\mathrm{mol} / \mathrm{L}\end{array}$ & $\begin{array}{c}\text { Hanford } \\
\mathrm{mol} / \mathrm{L}\end{array}$ & $\begin{array}{l}\text { Cs-Free } \\
\text { mol/L }\end{array}$ & $\begin{array}{c}\text { SRS-SDS } \\
\mathrm{mol} / \mathrm{L}\end{array}$ & $\begin{array}{c}\text { SDS-Spike } \\
\text { mol/L }\end{array}$ \\
\hline $\mathrm{Hg}(\mathrm{II})$ & $2.5 \times 10^{-7}$ & $2.1 \times 10^{-9}$ & $1.0 \times 10^{-3}$ & $1.0 \times 10^{-3}$ & - \\
\hline $\mathrm{La}(\mathrm{III})$ & - & $1.0 \times 10^{-5}$ & $1.0 \times 10^{-5}$ & $1.0 \times 10^{-5}$ & - \\
\hline $\mathrm{MnO}_{4}^{-}$ & - & $2.1 \times 10^{-5}$ & $3.8 \times 10^{-6}$ & $3.8 \times 10^{-6}$ & - \\
\hline $\mathrm{MoO}_{4}{ }^{2-}$ & $7.0 \times 10^{-5}$ & - & $7.0 \times 10^{-3}$ & $7.0 \times 10^{-3}$ & - \\
\hline $\mathrm{Ni}(\mathrm{II})$ & - & $3.3 \times 10^{-4}$ & $2.0 \times 10^{-6}$ & $2.0 \times 10^{-6}$ & - \\
\hline $\mathrm{NH}_{3}$ & $1.0 \times 10^{-3}$ & - & $8.1 \times 10^{-6}$ & $8.1 \times 10^{-6}$ & - \\
\hline $\mathrm{Pb}(\mathrm{II})$ & $1.0 \times 10^{-5}$ & $9.7 \times 10^{-5}$ & $3.0 \times 10^{-2}$ & $3.0 \times 10^{-2}$ & - \\
\hline $\operatorname{Pd}(\mathrm{II})$ & $3.8 \times 10^{-6}$ & - & $2.0 \times 10^{-5}$ & $2.0 \times 10^{-5}$ & - \\
\hline $\mathrm{PO}_{4}^{3-}$ & $7.0 \times 10^{-3}$ & $1.3 \times 10^{-2}$ & - & - & - \\
\hline $\mathrm{Rh}(\mathrm{III})$ & $2.0 \times 10^{-6}$ & - & $2.7 \times 10^{-5}$ & $2.7 \times 10^{-5}$ & - \\
\hline $\mathrm{Ru}(\mathrm{III})$ & $8.1 \times 10^{-6}$ & - & $1.9 \times 10^{-6}$ & $1.9 \times 10^{-6}$ & - \\
\hline $\mathrm{Si}(\mathrm{IV})$ & $3.0 \times 10^{-2}$ & $2.3 \times 10^{-3}$ & $1.2 \times 10^{-4}$ & $1.2 \times 10^{-4}$ & - \\
\hline $\operatorname{Sn}(\mathrm{II})$ & $2.0 \times 10^{-5}$ & - & $1.6 \times 10^{-4}$ & $1.6 \times 10^{-4}$ & - \\
\hline $\mathrm{Sr}^{2+}$ & - & $4.5 \times 10^{-6}$ & $3.3 \times 10^{-2}$ & $3.3 \times 10^{-2}$ & - \\
\hline $\mathrm{Zn}(\mathrm{II})$ & $1.2 \times 10^{-4}$ & - & $8.0 \times 10^{-3}$ & $8.0 \times 10^{-3}$ & - \\
\hline $\mathrm{Zr}(\mathrm{IV})$ & - & $2.4 \times 10^{-5}$ & $1.7 \times 10^{-4}$ & $1.7 \times 10^{-4}$ & - \\
\hline $\begin{array}{l}\text { Minor organic } \\
\text { constituents: }\end{array}$ & & & & & - \\
\hline$n$-Butanol & $2.7 \times 10^{-5}$ & - & $2.7 \times 10^{-5}$ & $2.7 \times 10^{-5}$ & - \\
\hline $\begin{array}{l}\text { Tri-n-butylphosphate } \\
\text { (TBP) }\end{array}$ & $1.9 \times 10^{-6}$ & - & $1.9 \times 10^{-6}$ & $1.9 \times 10^{-6}$ & - \\
\hline $\begin{array}{l}\text { Di-n-butylphosphate } \\
\text { (DBP) }\end{array}$ & $1.2 \times 10^{-4}$ & - & $1.2 \times 10^{-4}$ & $1.2 \times 10^{-4}$ & - \\
\hline $\begin{array}{l}\text { Mono-n- } \\
\text { butylphosphate (MBP) }\end{array}$ & $1.6 \times 10^{-4}$ & - & $1.6 \times 10^{-4}$ & $1.6 \times 10^{-4}$ & - \\
\hline Formate $\left(\mathrm{HCO}_{2}^{-}\right)$ & $3.3 \times 10^{-2}$ & - & $3.3 \times 10^{-2}$ & $3.3 \times 10^{-2}$ & - \\
\hline Oxalate $\left(\mathrm{C}_{2} \mathrm{O}_{4}{ }^{2-}\right)$ & $8.0 \times 10^{-3}$ & $1.0 \times 10^{-2}$ & $8.0 \times 10^{-3}$ & $8.0 \times 10^{-3}$ & - \\
\hline Trimethylamine & $1.7 \times 10^{-4}$ & - & $1.7 \times 10^{-4}$ & $1.7 \times 10^{-4}$ & - \\
\hline Sodium dodecyl sulfate & - & - & - & $1.0 \times 10^{-3}$ & $1.0 \times 10^{-3}$ \\
\hline
\end{tabular}

\subsubsection{Solvent Components}

Solvent components were obtained from commercial sources and judged to be of adequate purity for use as received. 1-(2,2,3,3-Tetrafluoropropoxy),-3-[4-(sec-butyl)phenoxy]-2-propanol (Cs7SB modifier) and MaxCalix were obtained from Marshallton Research Laboratories, and Isopar ${ }^{\mathbb{B}}$ L was obtained from ExxonMobil. The $N, N^{\prime}$-dicyclohexyl- $N^{\prime \prime}$-isotridecylguanidine suppressors DCiTG L, the active ingredient of a commercially available reagent supplied by Cognis and extracted at ORNL, and DCiTG-E (synthesized using EXXAL 13 feedstock, see Chapter 3), used in the studies detailed in this chapter were in the form of the $\mathrm{HCl}$ salts. Solvents were prepared by weighing appropriate amounts of extractant, modifier, and suppressor into volumetric flasks and diluting with Isopar $\mathrm{L}$ to the mark. The components are shown in Table 8.3. 
Table 8.3. BOBCalixC6 CSSX and NG-CSSX solvent components

\begin{tabular}{|c|c|c|c|}
\hline Component & Code & Chemical Name & Structure \\
\hline Extractant & MaxCalix & $\begin{array}{l}\text { 1,3-alt-25,27-Bis(3,7- } \\
\text { dimethyloctyl-1-oxy) } \\
\text { calix[4]arene- } \\
\text { benzocrown-6 }\end{array}$ & \\
\hline Extractant & BOBCalixC6 & $\begin{array}{l}\text { Calix[4] arene-bis(tert- } \\
\text { octylbenzocrown-6) }\end{array}$ & \\
\hline Modifier & Cs-7SB & $\begin{array}{l}\text { 1-(2,2,3,3- } \\
\text { Tetrafluoropropoxy)-3- } \\
\text { (4-sec-butylphenoxy)- } \\
\text { 2-propanol }\end{array}$ & \\
\hline Suppressor* & DCiTG & $\begin{array}{l}N, N \text {-Dicyclohexyl- } N " \text { - } \\
\text { isotridecylguanidine }\end{array}$ & \\
\hline Suppressor & TOA & Tri- $n$-octylamine & \\
\hline Diluent & Isopar L & $\begin{array}{l}\text { C12-isoparaffinic } \\
\text { hydrocarbon }\end{array}$ & \\
\hline
\end{tabular}

*Various suppressors of the same nature were used throughout the studies in this chapter, these include the active agent of the LIX 79 (DCiTG L), a commercially available reagent supplied by Cognis; DCiTG-E, a reagent synthesized at ORNL; and DCiTG-M1 and DCiTG-M2, two batches of suppressor synthesized using different conditions by Marshallton Research Laboratories (see also Chapter 6).

\subsection{TEMPERATURE DEPENDENCE}

\subsubsection{Introduction}

Under funding from the DOE-EM Office of Technology Innovation and Development, recent laboratory efforts are being directed toward developing the NG-CSSX process for cesium removal from alkaline tank wastes ${ }^{1}$. In support of EM's drive to accelerate processing of tank wastes at the SRS and Hanford site $^{2}$, the laboratory efforts have sought increased performance of NG-CSSX, both in decontamination efficiency and waste-processing rate ${ }^{3}$. The BOBCalixC6 CSSX process currently operating in the MCU is temperature dependent ${ }^{33}$, necessitating process temperature control, set at $23 \pm 3{ }^{\circ} \mathrm{C}$ on extraction and $33 \pm 3{ }^{\circ} \mathrm{C}$ on stripping ${ }^{30}$. The temperature 
control improves reliability but also enhances flowsheet performance by simultaneously boosting extraction and stripping strength, resulting in overall higher decontamination of the waste. Given that the chemistry of NG-CSSX makes use of a related calixarene extractant, MaxCalix ${ }^{3}$, a significant temperature dependence for NG-CSSX performance is anticipated, though likely not exactly the same. By comparison with BOBCalixC6 CSSX, the calixarene concentration is much higher $(0.050 \mathrm{M})$ in the NG-CSSX process solvent, and the modifier concentration has been decreased to $0.5 \mathrm{M}$. In addition, a guanidine suppressor is used instead of tri-n-octylamine, and the aqueous scrub and strip solutions have been changed respectively to $0.025 \mathrm{M} \mathrm{NaOH}$ and $0.010 \mathrm{M} \mathrm{H}_{3} \mathrm{BO}_{3}$. These substantial changes to the flowsheet chemistry make it important to quantify the temperature dependence of the $D_{\mathrm{Cs}}$ under expected flowsheet conditions.

This section provides data enabling prediction and control of NG-CSSX performance by controlling process temperatures. As before ${ }^{7,33}$, the approach taken was to employ ESS batch tests using process solutions at anticipated process organic:aqueous $(\mathrm{O}: \mathrm{A})$ phase volume ratios ${ }^{3}$.

\subsubsection{Experimental Section}

\subsubsection{Materials}

Solvent Components. Solvent components were obtained from commercial sources and judged to be of adequate purity for use as received. $N, N$-Dicyclohexyl- $N "$-isotridecylguanidine, the active guanidine reagent in LIX 79, was supplied by Cognis. Cs-7SB modifier and MaxCalix were obtained from Marshallton Research, and Isopar L was obtained from ExxonMobil. Solvents were prepared by weighing appropriate amounts of extractant, modifier, and suppressor (LIX 79 guanidine) a volumetric flask and diluting with Isopar L to the mark.

Waste Tank Simulant Composition. The nominal SRS-15 tank-waste simulant composition targeted is given in Table 8.1 according to the preparative method described in detail previously $^{3,7,9}$ and in Chapter 2. Appropriate amounts of reagent-grade chemicals were weighed into volumetric flasks. Actual component concentrations therefore may differ from the concentrations tabulated ${ }^{7}$. The SRS-15 simulant is designed to represent the average SRS tankwaste composition ${ }^{9}$.

\subsubsection{Methods}

The solvent was comprised of 0.050 M MaxCalix, 0.5 M Cs-7SB modifier, and 0.003 M LIX 79 guanidine in Isopar L, which is the formulation selected previously for NG-CSSX process scale- 
up and testing ${ }^{3}$. The solvent was prepared and prewashed prior to use in the following manner $(\mathrm{O}: \mathrm{A}=1)$ : one contact sequentially with $0.010 \mathrm{M} \mathrm{HCl}, \mathrm{H}_{2} \mathrm{O}$, then decreasing concentrations of $\mathrm{NaOH}(0.3 \mathrm{M}, 0.1 \mathrm{M}, 0.03 \mathrm{M}$, and $0.01 \mathrm{M})$, and then once or twice with $\mathrm{H}_{2} \mathrm{O}$ until the solution was $\mathrm{pH}$ neutral.

$D_{\mathrm{Cs}}$ with simulants were obtained in a manner similar to that described in Chapter 2, with $\mathrm{ES}_{2} \mathrm{~S}_{3}$ sequence. Phases were contacted in polypropylene micro-tubes mounted by clips on a disk rotated for 60 minutes for extractions and 45 minutes for scrubs and strips. Subsequently, the vials were centrifuged for 5 minutes at 3000 RPM at the same temperature used for the equilibrations. The solutions were contacted at varying temperature at 10-degree increments as before ${ }^{33}: 15,25$, and $35{ }^{\circ} \mathrm{C}$. The contacts were carried out in different chambers: Fisher Scientific Low Temperature Incubator $\left(15 \pm 1{ }^{\circ} \mathrm{C}\right)$, custom-made air box $\left(25 \pm 0.2{ }^{\circ} \mathrm{C}\right)$, and Lab Line Imperial III Incubator $\left(35 \pm 2{ }^{\circ} \mathrm{C}\right)$. An appropriate aliquot of each phase was subsampled and counted using a Packard Cobra II Auto-Gamma counter. A spike of Cs-137 was added to the second and third aqueous strip solutions, owing to the low number of counts remaining after each strip. To keep samples at the equilibration temperature, tubes were removed individually from the temperature-controlled centrifuge for subsampling. $D_{\mathrm{Cs}}$ are given as the ratio of the background-corrected volumetric count rates of the radioisotope in each phase at equilibrium.

\subsection{SODIUM EFFECTS}

\subsubsection{Introduction}

The original BOBCalixC6 CSSX process was optimized ${ }^{7,31,33}$ to treat waste feed at $5.6 \mathrm{M}$ sodium. In effect, the simple concept of increasing the sodium concentration of the feed amounts to faster overall processing by increasing the mass of waste per gallon of feed. This option affects both upstream and downstream processing and therefore can only be properly evaluated from a system-wide standpoint. However, an obvious starting point is to understand the impact of sodium concentration within the NG-CSSX process itself, and accordingly, that is the focus of the limited study reported herein.

Prior data from chemical development lends optimism that the process is robust to changes in sodium concentration in the feed. It is known that the $D_{\mathrm{Cs}}$ lie on a plateau with respect to aqueous sodium nitrate concentration in the range $1-5 \mathrm{M}$, a broad maximum occurring at $\sim 3 \mathrm{M} \mathrm{NaNO}_{3}{ }^{23}$. The dependence of $D_{\mathrm{Cs}}$ on aqueous sodium concentration exhibits a monotonic, concave-up 
curve. Thus, the effect of increased sodium concentration is either neutral, slightly negative if increased as nitrate, or positive if increased as hydroxide. Comparisons of predicted against experimental $D_{\mathrm{Cs}}$ values show good agreement and demonstrate a robustness of $D_{\mathrm{Cs}}$ for a limited range of sodium concentrations, 3.3-6.2 M, for actual and simulated SRS tank wastes ${ }^{19,23}$ and simulated Hanford wastes ${ }^{8,10}$. Notwithstanding the sensitivity of $D_{\mathrm{Cs}}$ to potassium concentration, an issue at Hanford but not $\mathrm{SRS}^{3,8,10}$, the effect of sodium concentration is not pronounced. A promising batch NG-CSSX performance test on Tank $49 \mathrm{H}$ real waste at $6.8 \mathrm{M}$ sodium gave a temperature-corrected $D_{\mathrm{Cs}}$ value of 61.8 at $25{ }^{\circ} \mathrm{C}^{3,24}$, which compares well with a $D_{\mathrm{Cs}}$ value of 51.1 for average SRS simulant at $25^{\circ} \mathrm{C}^{3}$.

This section examines the impact of sodium concentration on the extraction strength of the NGCSSX solvent. Specifically, batch ESS tests were employed to assess the ability of the NG-CSSX solvent to maintain its extraction strength and good stripping with elevated sodium levels in the feed. Impacts to hydraulic properties are deferred to proposed follow-on efforts. The reference NG-CSSX solvent, aqueous solutions, and O:A ratios used in the tests are the same as those reported recently ${ }^{3}$. A series of aqueous feed solutions were prepared based upon a global average waste known as SRS-15 $5^{3,7}$. Within this series, the total sodium concentration was varied in the range 3.5-8.0 $\mathrm{M}$ with all components changing in proportion to the sodium concentration. This approach models the simple situation that the average waste is more or less concentrated, but it likely does not model the changing feed conditions from early to late retrieval of salt cake.

\subsubsection{Experimental Section}

\subsubsection{Materials}

Solvent Components. See 8.2.2.1

Tank-Waste Simulant Composition. Tank-waste simulant compositions used herein are given in Table 8.1. Constituent species are named in the table as the chemical forms added, not necessarily as the forms actually present after mixing. Appropriate amounts of reagent-grade chemicals were weighed into volumetric flasks in the order prescribed to prepare the SRS-15 and SRS-22 simulants. Significant precipitate formed upon adding the $\mathrm{NaOH}$, which is considered normal, and the mixtures were stirred for several hours and allowed to settle for a few days before use. Consequently, actual component concentrations may differ from the concentrations tabulated $^{7}$. The SRS-15 simulant $^{7}$, designed to represent the average SRS tank-waste 
composition $^{9}$, was diluted to make the two simulants containing 3.5 and $4.5 \mathrm{M}$ sodium, respectively. The $8 \mathrm{M} \mathrm{Na}^{+}$waste tank simulant (SRS-22) is a modification of the SRS-15 simulant wherein all major components have been increased by a factor of 1.43 to achieve the desired $\mathrm{Na}^{+}$concentration. The SRS-19 simulant is a dilution of SRS-22.

\subsubsection{Methods}

One solvent comprised of 0.05 M MaxCalix, 0.5 M Cs-7SB modifier, and 0.003 M LIX 79 guanidine in Isopar L was used during the ESS experiments. The solvent was prepared and washed prior to use in the following manner: one contact sequentially with $0.010 \mathrm{M} \mathrm{HCl}, \mathrm{H}_{2} \mathrm{O}$, then with decreasing concentrations of $\mathrm{NaOH}(0.3 \mathrm{M}, 0.1 \mathrm{M}, 0.03 \mathrm{M}$, and $0.01 \mathrm{M})$, and then once or twice with $\mathrm{H}_{2} \mathrm{O}$ until the solution was $\mathrm{pH}$ neutral.

$D_{\mathrm{Cs}}$ with simulants were obtained in a manner similar to that described in Chapter 2. See 8.2.2.2 for further specific details.

Waste simulant densities were determined gravimetrically in duplicate by use of $1 \mathrm{~mL}$ volumetric flasks at $25 \pm 0.2{ }^{\circ} \mathrm{C}$. Duplicate agreement decreased as the density increased, which is attributed to the observed increasingly uneven meniscus of the solution in the neck of the volumetric flask. Duplicate agreement was taken as a basis for the precision of the measurements down to a minimum of \pm 0.012 , corresponding to the expected propagated error based on the contributing tolerances due to the balance, volumetric glassware, and small temperature variation $\left( \pm 0.2^{\circ} \mathrm{C}\right)$.

\subsection{EFFECT OF POTASSIUM AND CESIUM CONCENTRATION}

\subsubsection{Introduction}

The potential application of the NG-CSSX solvent to remove cesium from Hanford waste types is highly dependent on the solvents ability to selectively extract cesium in the presence of higher concentrations of potassium. Hanford waste types are more challenging than those currently being treated at Savannah River, as they contain significantly higher concentrations of potassium. In the initial BOBCalixC6 CSSX process a computational model was developed and it showed that the higher levels of potassium present in the Hanford waste would significantly suppress cesium removal ${ }^{10}$. 


\subsubsection{Experimental Section}

\subsubsection{Materials}

Solvent Components. Solvent components were obtained from commercial sources and judged to be of adequate purity for use as received. 1-(2,2,3,3-Tetrafluoropropoxy),-3-[4-(secbutyl)phenoxy]-2-propanol (Cs-7SB modifier, Lot No. MOD2010-M-2) and 1,3- alt-25,27bis(3,7-dimethyloctyl-1-oxy)calix[4]arene-benzocrown-6 (MaxCalix, Lot No. 71-061-15) were obtained from Marshallton Research Laboratories, and Isopar L (Lot No. US67377A) was obtained from ExxonMobil. The $N, N^{\prime}$-dicyclohexyl- $N^{\prime \prime}$-isotridecylguanidine suppressors DCiTG $\mathrm{L}$, was in the form of the $\mathrm{HCl}$ salt. Solvent was prepared by weighing appropriate amounts of extractant, modifier, and suppressor into volumetric flasks and diluting with Isopar $L$ to the mark. The components are shown in Table 8.2.

Aqueous Solution Preparation. The targeted composition of the SRS-15 tank-waste simulant used in the majority of the extraction studies is given in Table 8.1 according to the preparative method described in detail in Chapter 2. SRS-45 and Hanford simulant componants are given in Table 8.3. Constituent species are named in the table as the chemical forms added, not necessarily to be taken as the forms actually present after mixing. Appropriate amounts of reagent-grade chemicals were weighed into volumetric flasks. Tracer ${ }^{137} \mathrm{Cs}$ was added to the simulant only for ESS tests as described above. Scrub solution $(0.025 \mathrm{M} \mathrm{NaOH})$ and other $\mathrm{NaOH}$ solutions were prepared by dilution of $1.0 \mathrm{M} \mathrm{NaOH}$ standard solution (VWR). Strip solution (0.010 $\left.\mathrm{M} \mathrm{H}_{3} \mathrm{BO}_{3}\right)$ was obtained by dilution of a $0.1 \mathrm{M} \mathrm{H}_{3} \mathrm{BO}_{3}$ stock solution prepared from lab-grade $\mathrm{H}_{3} \mathrm{BO}_{3}$ (min 95.5\%, Sigma Aldrich). Solutions of $\mathrm{HCl}$ were made from $1.0 \mathrm{M} \mathrm{HCl}$ standard solution (Baker). Water for preparation of all aqueous solutions was first distilled and then deionized using a Milli$\mathrm{Q}^{\circledR}$ gradient A10 filtering system equipped with a Quantu Ex Ultrapure Organex Cartridge $\left(18.2 \mathrm{M} \Omega \cdot \mathrm{cm}\right.$ at $25^{\circ} \mathrm{C}$, total organic content $\left.4 \mathrm{ppb}\right)$.

\subsubsection{Methods}

Cesium distribution determination using gamma counting. $D_{\mathrm{Cs}}$ with simulants were obtained in a manner similar to that described previously ${ }^{3}$, with $\mathrm{ES}_{2} \mathrm{~S}_{3}$ sequence. For each experiment the solvents were prepared and then prewashed prior to use in the following manner: one contact sequentially with $0.010 \mathrm{M} \mathrm{HCl}, \mathrm{H}_{2} \mathrm{O}$, then decreasing concentrations of $\mathrm{NaOH}(0.3 \mathrm{M}, 0.1 \mathrm{M}$, $0.03 \mathrm{M}$, and $0.01 \mathrm{M}$ ), and then once or twice with $\mathrm{H}_{2} \mathrm{O}$ until the solution was $\mathrm{pH}$ neutral. Phases were contacted in polypropylene micro-tubes mounted by clips on a disk rotated for 60 minutes 
for extractions and 45 minutes for scrubs and strips. Subsequently, the vials were centrifuged for 5 minutes at 3000 RPM at the same temperature used for the equilibrations. The contacts were carried out in different chambers: Fisher Scientific Low Temperature Incubator $\left(15 \pm 1{ }^{\circ} \mathrm{C}\right)$, custom-made air box $\left(25 \pm 0.2^{\circ} \mathrm{C}\right)$, and Lab Line Imperial III Incubator $\left(35 \pm 2{ }^{\circ} \mathrm{C}\right)$. An appropriate aliquot of each phase was subsampled and counted using a Packard Cobra II AutoGamma counter. A spike of Cs-137 was added to the second and third aqueous strip solutions, owing to the low number of counts remaining after each strip. To keep samples at the equilibration temperature, tubes were removed individually from the temperature-controlled centrifuge for subsampling. The precision of $D_{\mathrm{Cs}}$ values is estimated to be $\pm 5 \%$ (extraction and scrub), $\pm 10 \%$ (first strip), and $\pm 30 \%$ (second and third strips, which have very low organic-phase counts). $D_{\mathrm{Cs}}$ are given as the ratio of the background-corrected volumetric count rates of the radioisotope in each phase at equilibrium.

\subsection{DETERMINING AQUOUS FEED ALKALINE METAL CONCENTRATION USING INDUCTIVELY COUPLED PLASMA ATOMIC EMISSION SPECTROSCOPY (ICP-AES)}

\subsubsection{Introduction}

The effects of varying the alkaline metal concentrations in the waste simulant can be observed via ESS testing, which also allows us to calculate the concentration of cesium in each of the aqueous feeds. It is also important, however, to understand the fate of the sodium and potassium ions that are extracted together with the cesium. Although ${ }^{22} \mathrm{Na}$ could potentially be used to track sodium the low partitioning of sodium into the NG-CSSX solvent together with the very high concentration of $\mathrm{Na}$ in the waste simulant make the analyses more complex. ICP-AES has been used to directly analyze the potassium and sodium concentrations in the scrub and strip solutions of a benchtop ESS.

\subsubsection{Experimental Section}

\subsubsection{Materials}

Solvent componants. The solvent used in these experiments comprised of MaxCalix (0.05 M), Cs-7SB modifier $(0.5 \mathrm{M})$, and DCiTG L guanidine suppressor $(0.003 \mathrm{M})$ in Isopar L. The solvent was prepared as described above and prewashed prior to use in the following manner: sequential contacts (single contact with an $\mathrm{O}: \mathrm{A}$ of $1: 1$ ) with $0.010 \mathrm{M} \mathrm{HCl}, \mathrm{H}_{2} \mathrm{O}$, then decreasing 
concentrations of $\mathrm{NaOH}(0.3 \mathrm{M}, 0.1 \mathrm{M}, 0.03 \mathrm{M}$, and $0.01 \mathrm{M})$, and then with $\mathrm{H}_{2} \mathrm{O}$ until the solution was $\mathrm{pH}$ neutral.

Aqueous Solution Preparation. The targeted composition of the SRS-15 tank-waste simulant used in the majority of the extraction studies is given in Table 8.1 according to the preparative method described in detail in Chapter 2. SRS-45 and Hanford simulant componants are given in Table 8.3. Constituent species are named in the table as the chemical forms added, not necessarily to be taken as the forms actually present after mixing. Appropriate amounts of reagent-grade chemicals were weighed into volumetric flasks. Tracer ${ }^{137} \mathrm{Cs}$ was added to the simulant only for ESS tests as described above. Scrub solution $(0.025 \mathrm{M} \mathrm{NaOH})$ and other $\mathrm{NaOH}$ solutions were prepared by dilution of $1.0 \mathrm{M} \mathrm{NaOH}$ standard solution (VWR). Strip solution $\left(0.010 \mathrm{M} \mathrm{H}_{3} \mathrm{BO}_{3}\right)$ was obtained by dilution of a $0.1 \mathrm{M} \mathrm{H}_{3} \mathrm{BO}_{3}$ stock solution prepared from lab-grade $\mathrm{H}_{3} \mathrm{BO}_{3}$ (min 95.5\%, Sigma Aldrich). Solutions of $\mathrm{HCl}$ were made from $1.0 \mathrm{M} \mathrm{HCl}$ standard solution (Baker). Water for preparation of all aqueous solutions was first distilled and then deionized using a MilliQ gradient A10 filtering system equipped with a Quantum Ex Ultrapure Organex Cartridge $\left(18.2 \mathrm{M} \Omega \cdot \mathrm{cm}\right.$ at $25^{\circ} \mathrm{C}$, total organic content $\left.4 \mathrm{ppb}\right)$.

\subsubsection{Methods}

ESS analysis using each of the waste simulants was carried out in a manner similar to that described earlier, after the contacting period, the tubes were centrifuged for 5 minutes at 3000 RPM at the experimental temperature. The aqueous and organic phases from the scrub and strip stages were separated and the aqueous phases were subsampled $(1.0 \mathrm{~mL})$ and diluted ten-fold with deionized water for ICP analysis.

An IRIS Intrepid II XSP Inductively Coupled Plasma Atomic Emission spectrometer was used for sample analysis using the Thermo elemental TEVA® 2001 software. The machine was calibrated using standards purchased from High Purity Standards. Mixed element standards comprised of sodium, potassium, boron, phosphorus, sulfur and aluminum at 1, 2.5, 5, 7.5, 10, 25, 50,100 and $500 \mathrm{ppm}$ were prepared $(50 \mathrm{~mL}$ in deionized water). The wavelengths used for each element are given in Table 8.4 along with the correlation, $\mathrm{R}^{2}$, of the calibration curve obtained. 
Table 8.4. Wavelengths used for ICP-AES analysis given along with the correlation of the calibration curve for each analyte and the precision of the measurements $<10 \mathrm{ppm}$

\begin{tabular}{lccc}
\hline Element & Wavelength $(\mathbf{n m})$ & Calibration $\mathbf{R}^{\mathbf{2}}$ & Observed precision at $<\mathbf{1 0} \mathbf{p p m}$ \\
\hline $\mathrm{Na}$ & 589.592 & 0.999 & $\pm 0.50 \mathrm{ppm}$ \\
$\mathrm{K}$ & 766.491 & 0.999 & $\pm 0.10 \mathrm{ppm}$ \\
$\mathrm{B}$ & 249.773 & 0.999 & $\pm 0.25 \mathrm{ppm}$ \\
$\mathrm{S}$ & 180.731 & 0.999 & $\pm 0.02 \mathrm{ppm}$ \\
$\mathrm{P}$ & 177.499 & 0.999 & $\pm 0.02 \mathrm{ppm}$ \\
$\mathrm{Al}$ & 308.215 & 0.999 & $\pm 0.25 \mathrm{ppm}$ \\
\hline
\end{tabular}

Each experiment was done in duplicate and the analysis of each sample was carried out in quadruplicate.

\subsection{THIRD PHASE FORMATION AND INTERFACIAL TENSION MEASUREMENTS}

\subsubsection{Introduction}

Whilst investigating the effect of concentration of the boric acid strip solution it was observed that upon a third contact with high concentrations of $\mathrm{H}_{3} \mathrm{BO}_{3}(0.1 \mathrm{M}$ at a O:A of 3.75:1), followed by $1 \mathrm{~min}$ hand vortexing and 5 min centrifuge, the NG-CSSX solvent results forms a white emulsion (see Fig. 8.1). It was later confirmed that a relationship existed between the concentration of the boric acid strip solution and the concentration of the guanidine suppressor in the NG-CCX solvent.

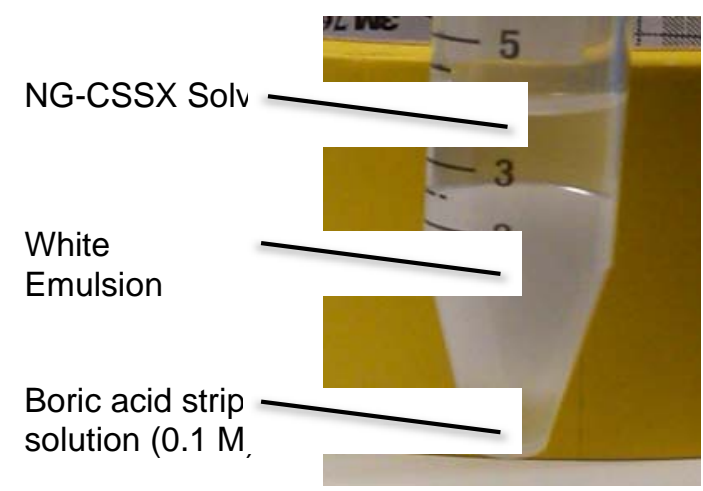

Fig. 8.1. Emulsion formed upon hand vortexing of the NG-CSSX solvent with boric acid (0.1 M) at an $0:$ A of $3.75: 1$. 


\subsubsection{Experimental Section}

\subsubsection{Materials}

Solutions of the NG-CSSX solvent (MaxCalix, 0.05 M; Cs7SB, 0.5 M in Isopar L diluent) with varying concentrations of the guanidine suppressor (DCiTG L; 0, 0.003, 0.005, 0.01, .02 and 0.03 M) were contacted with solutions of boric acid (0, 0.002, 0.005, 0.01, 0.025, 0.05, 0.1, 0.5 and 1.0 M) at an O:A of 3.75:1. Each solution was hand vortexed for 1 minute and centrifuged for 5 minutes. Confirmation of the presence of an emulsion was done visually.

\subsubsection{Methods}

Interfacial tension measurements. Interfacial tension measurements were made with a FT-9435 DuNouy tensiometer using the DuNouy ring method ${ }^{22}$ (platinum-iridium rings; $6 \mathrm{~cm}$ ). The solvent (MaxCalix, 0.05 M; Cs7SB, 0.5 M in Isopar L diluent) with varying concentrations of the guanidine suppressor (DCiTG L; 0, 0.003, and 0.03 M) was pre-equilibrated with the aqueous solutions of boric acid $(0,0.01$, and $0.05 \mathrm{M})$ for 30 minutes inside of an air-box maintained at a temperature of $25 \pm 0.2{ }^{\circ} \mathrm{C}$. After the contacting period, the tubes were centrifuged for 5 minutes at $3000 \mathrm{RPM}$ at the experimental temperature prior to separation.

A fresh sample of the aqueous solution $(30 \mathrm{ml})$ was pipetted into a clean glass beaker $(100 \mathrm{ml})$ and the pre-flamed platinum-iridium ring was positioned below the surface. The organic layer (30 $\mathrm{ml}$ ) was carefully pipetted onto the aqueous taking care not to disturb the interface. Each experimental variation was carried out in duplicate and the interfacial tension was measured in triplicate for each sample.

Emulsion breaking. Solutions of the NG-CSSX solvent (MaxCalix, 0.05 M; Cs7SB, 0.5 M; DCiTG L, 0.02 M, in Isopar L diluent) were contacted with of boric acid (0.1 M) at an O:A of 3.75:1. Each solution was vortexed for 1 minute and centrifuged for 5 minutes. The remaining aqueous layer was removed and the new "stripping" agent was added to the emulsion and organic layer. The solutions were, when necessary, hand vortexed for 1 minute and centrifuged for 5 minutes. Confirmation of emulsion removal was done visually. 


\subsection{RESULTS AND DISCUSSION}

\subsubsection{Effect of Sodium on Distribution of Cesium}

The effect of the concentration of sodium on $D_{C s}$ in a standard $\mathrm{ES}_{2} \mathrm{~S}_{3}$ batch test with NG-CSSX solvent is given in Table 8.5. A slight rise in $D_{C s}$ with increasing sodium concentration and is observed for all stages. Enhanced extraction is due to increasing nitrate and hydroxide ${ }^{19,23}$ in the simulant, with the hydroxide being sufficiently dominant that the nitrate plateau is not observed. Although the amount of potassium is still too low to significantly load the solvent, its increasing concentration would tend to mildly depress $D_{C s}$ on extraction. The observed trend of increasing $D_{C s}$ in the scrub stages with feed sodium concentration increases is considered a direct effect of greater sodium and potassium loading during the extraction stage. These metal cations are released into the scrub aqueous phase, raising its nitrate and hydroxide concentration, thereby raising the $D_{C s}$ value by mass-action. On stripping, increased cesium loading of the solvent, along with a slight amount of remaining potassium is likely responsible for the mild increasing trend in $D_{C s}$. Even so, the boric acid still has the capacity to effectively remove the cesium from the solvents contacted with simulants containing higher sodium levels.

As a general observation, the impact of increased waste concentration in the feed is expected to slightly enhance flowsheet performance, other factors remaining the same. Under the assumption that hydraulics and stage efficiency remain constant, an increase in $D_{C S}$ in extraction implies an increased DF in a given configuration such as the MCU. The increases in scrub and strip $D_{C s}$ values are minor and of little consequence for overall flowsheet performance. Stripping is already performing much better than needed, and thus, considerable increase in $D_{C s}$ can be tolerated.

Table 8.5. ESS performance of the NG-CSSX solvent with varying sodium concentration in SRS simulants ${ }^{a}$

\begin{tabular}{|c|c|c|c|c|c|c|c|}
\hline \multirow[t]{2}{*}{ Step } & \multirow[t]{2}{*}{$\begin{array}{l}\text { Aqueous } \\
\text { phases }\end{array}$} & \multirow[t]{2}{*}{$\mathbf{O}: \mathbf{A}^{b}$} & \multicolumn{5}{|c|}{$D_{\mathrm{Cs}}$} \\
\hline & & & $3.5 \mathrm{M} \mathrm{Na}^{+}$ & $4.5 \mathrm{M} \mathrm{Na}^{+}$ & $5.6 \mathrm{M} \mathrm{Na}^{+}$ & $6.8 \mathrm{M} \mathrm{Na}^{+}$ & $8.0 \mathrm{M} \mathrm{Na}^{+}$ \\
\hline Extraction & $\begin{array}{c}\text { SRS } \\
\text { Simulant }^{c} \\
0.025 \mathrm{M}\end{array}$ & $1: 4$ & $35.2(2)$ & $42.0(3)$ & $50(1)$ & $54(5)$ & $66(5)$ \\
\hline Scrub 1 & $\mathrm{NaOH}$ & $3.75: 1$ & $1.36(1)$ & $1.70(4)$ & $2.09(6)$ & $3.38(1)$ & $3.48(3)$ \\
\hline Scrub 2 & “ & $3.75: 1$ & $0.4(5)$ & $0.787(5)$ & $0.85(1)$ & $1.21(4)$ & $1.4(1)$ \\
\hline $\begin{array}{l}\text { Strip } 1 \\
\text { Strip } 2 \\
\text { Strip } 3\end{array}$ & $\begin{array}{c}0.010 \mathrm{M} \\
\mathrm{H}_{3} \mathrm{BO}_{3} \\
\text { ، } \\
\text { ، }\end{array}$ & $\begin{array}{l}3.75: 1 \\
3.75: 1 \\
3.75: 1\end{array}$ & $\begin{array}{r}5.8(2) \times 10^{-4} \\
9(6) \times 10^{-5} \\
2(1) \times 10^{-4}\end{array}$ & $\begin{array}{c}5(2) \times 10^{-4} \\
1(2) \times 10^{-4} \\
0.5 \times 10^{-4}\end{array}$ & $\begin{array}{c}1.1(1) \times 10^{-3} \\
1.1(4) \times 10^{-4} \\
3(3) \times 10^{-4}\end{array}$ & $\begin{array}{l}2.57(0) \times 10^{-3} \\
3(2) \times 10^{-4} \\
6(5) \times 10^{-4}\end{array}$ & $\begin{array}{l}2.9(5) \times 10^{-3} \\
4(1) \times 10^{-4} \\
3.7(6) \times 10^{-4}\end{array}$ \\
\hline
\end{tabular}


Table 8.5. ESS performance of the NG-CSSX solvent with varying sodium concentration in SRS simulants ${ }^{a}$

\begin{tabular}{|c|c|c|c|c|c|}
\hline \multirow[t]{2}{*}{ Step } & \multirow[t]{2}{*}{$\begin{array}{l}\text { Aqueous } \\
\text { phases }\end{array}$} & \multirow[t]{2}{*}{$O: A^{b}$} & \multicolumn{3}{|c|}{$D_{\mathrm{Cs}}$} \\
\hline & & & $\begin{array}{ll}3.5 \mathrm{M} \mathrm{Na}^{+} & 4.5 \mathrm{M} \mathrm{Na}^{+}\end{array}$ & $\begin{array}{ll}5.6 \mathrm{M} \mathrm{Na}^{+} & 6.8 \mathrm{M} \mathrm{Na}^{+}\end{array}$ & $8.0 \mathrm{M} \mathrm{Na}^{+}$ \\
\hline
\end{tabular}

\subsubsection{Density of Simulants}

The density of each simulant prepared was determined at a modest level of precision (Table 8.6). Such data may be used as initial input for estimating contactor performance and weir diameter. A density increase is observed as sodium concentration increases. As an isolated effect, this parameter increase would be expected to increase phase dispersion numbers and contactor throughput. However, dispersion numbers and contactor hydraulics are subject to interfacial properties that cannot be readily predicted, and therefore actual contactor testing is needed for reliable conclusions to be drawn.

Table 8.6. Densities of SRS simulants used in the experiment ${ }^{a}$

\begin{tabular}{cccccc}
\hline & \multicolumn{5}{c}{${\text { Density }(\mathbf{g} / \mathbf{m L})^{\boldsymbol{b}}}$} \\
\cline { 2 - 6 } & SRS-9 & SRS-12 & SRS-15 & SRS-19 & SRS-22 \\
\hline Trial 1 & 1.157 & 1.208 & 1.228 & 1.287 & 1.327 \\
Trial 2 & 1.159 & 1.199 & 1.248 & 1.312 & 1.293 \\
Average & $1.158 \pm 0.001$ & $1.204 \pm 0.005$ & $1.24 \pm 0.01$ & $1.30 \pm 0.02$ & $1.31 \pm 0.02$ \\
\hline
\end{tabular}

${ }^{a}$ The simulant compositions are given in Table 8.1.

${ }^{b}$ Density determinations were carried out in $1 \mathrm{~mL}$ volumetric flasks at $25^{\circ} \mathrm{C}$.

\subsubsection{Implications for Waste Decontamination and Processing Rat}

The ability of the NG-CSSX solvent to perform well in batch testing with higher sodium concentrations is a positive indication that the MCU and SWPF may be able to handle feeds with increased concentrations of sodium without a tradeoff in waste decontamination. Since the achievable DF depends upon stage efficiency, however, contactor testing will be required to establish whether an increase in waste decontamination can be realized in practice.

Whether an increase in sodium concentration actually results in increased waste-processing rate depends also upon contactor hydraulics. Since the feed density increases with increasing sodium concentration, hydraulics would ordinarily be expected to improve. However, other factors such as interfacial tension and aqueous viscosity also play a role; thus, contactor hydraulic testing is needed. 
If NG-CSSX can ultimately perform adequately with increased waste concentration in the feed, impacts to upstream and downstream processing must also be appreciated. Such impacts would include retrieval, actinide/strontium removal, and filtration on the upstream side as well as saltstone production on the downstream side. An understanding of the stability of the aqueous solution to precipitation of solids will also be needed for the aqueous stream through all operations. Since the current MCU operations are plagued by low filtration rates and precipitation of solids $s^{25,31}$, the impact of higher saturated salt solutions may be appreciable on the overall system attainment. Saltstone production similarly suffers currently from precipitation and hardening of the grout in the processing lines. A change in formulation requires study before declaring a more concentrated flowsheet as viable or advantageous.

\subsection{EFFECT OF CESIUM CONCENTRATION ON CESIUM DISTRIBUTION VALUES}

The effect of varying the total cesium concentration in the waste simulants on $D_{C s}$ in a standard $\mathrm{ES}_{2} \mathrm{~S}_{3}$ batch test with NG-CSSX solvent is given in Table 8.7. The extraction and scrubbing $D_{C s}$ values showed little change with increased cesium concentration, the stripping $D_{C s}$ values, however, have been shown to increase. The first and second stripping $D_{C s}$ values vary considerably but, by the third stripping stage, the $D_{C s}$ values have normalized.

Table 8.7. ESS performance of the NG-CSSX as cesium concentration in the simulant is increased $^{a}$

\begin{tabular}{|c|c|c|c|c|c|}
\hline Stage & $0.14 \mathrm{mM}$ & $0.25 \mathrm{mM}$ & $0.5 \mathrm{mM}$ & $0.75 \mathrm{mM}$ & $1.0 \mathrm{mM}$ \\
\hline Extraction & $52.1(7)$ & $49(2)$ & $50.8(3)$ & $48.6(2)$ & $48(1)$ \\
\hline Scrub 1 & $2.4(3)$ & $2.09(1)$ & $1.8(2)$ & $1.97(2)$ & $1.97(8)$ \\
\hline Scrub 2 & $0.91(0)$ & $0.867(2)$ & $0.9(1)$ & $0.89(2)$ & $0.92(6)$ \\
\hline Strip 1 & $9.4(5) \times 10^{-4}$ & $3.7(1) \times 10^{-3}$ & $1.9(9) \times 10^{-2}$ & $2.4(3) \times 10^{-2}$ & $4.1(2) \times 10^{-2}$ \\
\hline Strip 2 & $3.3(6) \times 10^{-4}$ & $2.2(2) \times 10^{-4}$ & $1(1) \times 10^{-3}$ & $2(1) \times 10^{-3}$ & $5(1) \times 10^{-3}$ \\
\hline Strip 3 & $8(4) \times 10^{-5}$ & $3(2) \times 10^{-4}$ & $6(2) \times 10^{-4}$ & $6(1) \times 10^{-4}$ & $7.6(8) \times 10^{-4}$ \\
\hline
\end{tabular}

\subsection{EFFECT OF CESIUM CONCENTRATION ON $D_{\mathrm{CS}}$}

The effect of different waste simulants, based on their potassium concentration, on the $D_{C s}$ in a standard $\mathrm{ES}_{2} \mathrm{~S}_{3}$ batch test with NG-CSSX solvent is given in Table 8.8. A decrease in the extraction $D_{C s}$ is observed with increased potassium concentration, due to the increasing 
competitive extraction of potassium. The $D_{C s}$ for the scrubbing stages show little change with increasing potassium concentration. The stripping $D_{C s}$ values, however, do decrease with increasing potassium concentration in the simulant. The cesium concentration in each of the SRSbased simulants is $0.14 \mathrm{mM}$ whereas the Hanford cesium concentration is less at $0.058 \mathrm{mM}$ (see Table 8.3). As shown above, the cesium concentration in the simulant has an effect on the stripping, the lower concentration of cesium in the Hanford waste together with the decreased extraction of cesium results observed in $D_{C s}$ stripping values decreasing with increasing simulant potassium concentration.

Table 8.8. ESS performance of the NG-CSSX as simulant changes, the major difference between simulants being the increasing potassium concentration $^{a}$

\begin{tabular}{|c|c|c|c|}
\hline $\begin{array}{c}\text { Simulant } \\
\left([\mathbf{K}]^{+}\right)\end{array}$ & $\begin{array}{c}\text { SRS-15 } \\
(0.015 \text { M) }\end{array}$ & $\begin{array}{c}\text { SRS-45 } \\
(0.045 \text { M) }\end{array}$ & $\begin{array}{c}\text { Hanford } \\
(0.174 \mathrm{M}) \\
\end{array}$ \\
\hline Extraction & $55.2(0.6)$ & $42.4(5)$ & $27.4(1)$ \\
\hline Scrub 1 & $2.57(.03)$ & $3.30(1)$ & $3.70(9)$ \\
\hline Scrub 2 & $1.033(1)$ & $1.30(4)$ & $1.00(1)$ \\
\hline Strip 1 & $2(1) \times 10^{-3}$ & $2.08(2) \times 10^{-3}$ & $6.1(9) \times 10^{-4}$ \\
\hline Strip 2 & $3.35(5) \times 10^{-4}$ & $4.4(8) \times 10^{-4}$ & $2.9(3) \times 10^{-4}$ \\
\hline Strip 3 & $2.8(4) \times 10^{-4}$ & $3.8(5) \times 10^{-4}$ & $2(1) \times 10^{-4}$ \\
\hline
\end{tabular}

\subsection{FOLLOWING THE ALKALINE METALS AND POSSIBLE IMPURITIES IN THE AQUEOUS FLOWSHEET USING INDUCTIVELY COUPLED PLASMA ATOMIC EMISSION SPECTROSCOPY (ICP-AES)}

From the results shown in Table 8.9, it has been seen that the uptake of sodium and potassium into the NG-CSSX solvent is directly related to the simulant composition and can be closely related to the variation in distribution values for cesium in the 'hot' $\mathrm{ES}_{2} \mathrm{~S}_{3}$ tests. The organic phosphate and sulfate impurities found in the majority of the simulants tested are shown to be extracted to a certain extent although appear to be almost completely removed in the scrubbing stages. The efficiency of the scrubbing stages is confirmed by the efficient removal of sodium and potassium even when the concentrations in the simulant are increased.

\subsubsection{Effect of Simulant on Sodium Concentration}

The levels of sodium seen in the scrubbing stages of the $\mathrm{ES}_{2} \mathrm{~S}_{3}$ seem to be indicative of the alkaline metal composition of the simulant. SRS-45 and Hanford show lower levels of sodium removed in scrubbing, which could be due to the higher concentrations of potassium in these 
simulants. When cesium is not present in the simulant the overall amount of sodium scrubbed appears to be higher.

\subsubsection{Effect of Simulant on Potassium Concentration}

The levels of potassium seen in the scrubbing stages of the $\mathrm{ES}_{2} \mathrm{~S}_{3}$ (Table 8.9) can also be related to the alkaline metal composition of the simulant. SRS-45 and Hanford show much higher levels of scrubbed potassium, due to the higher concentrations of potassium in these simulants $(0.045 \mathrm{~m}$ $\mathrm{L}^{-1}$ and $0.174 \mathrm{~m} \mathrm{~L}^{-1}$ for SRS-45 and Hanford respectively compared with 0.015 for SRS-15). When cesium is not present in the simulant the overall amount of potassium scrubbed appears to be marginally higher. When $\mathrm{DS}^{-}$is present, scrubbed potassium levels are slightly reduced. Potassium is removed from each of the solvents to a certain extent in the first strip stage of the $\mathrm{ES}_{2} \mathrm{~S}_{3}$; however, no significant potassium is removed from any of the contacted solvents on the second and third strip stages. When the lipophillic anion $\mathrm{DS}^{-}$is present, the level of potassium observed in stripping is higher, indicating either an enhanced extraction or perturbed scrubbing. 
Table 8.9. Showing the amount of $\mathrm{Na}, \mathrm{K}$ and Cs removed from the NG-CSSX solvent (in $\mathrm{mM}$ ) in the scrub and strip stages of the process after being contacted with the various simulants*

\begin{tabular}{|c|c|c|c|c|c|c|c|c|c|c|c|c|c|c|c|c|c|c|}
\hline \multirow{3}{*}{$\frac{\text { Simulant }}{\text { Element }}$} & \multirow{2}{*}{\multicolumn{3}{|c|}{ SRS-15 }} & \multirow{2}{*}{\multicolumn{3}{|c|}{ SRS-45 }} & \multirow{2}{*}{\multicolumn{3}{|c|}{ Hanford }} & \multirow{2}{*}{\multicolumn{3}{|c|}{ Cs-Free }} & \multirow{2}{*}{\multicolumn{3}{|c|}{ SDS }} & \multicolumn{3}{|c|}{ S, K, TM, Org Free - SDS } \\
\hline & & & & & & & & & & & & & & & & & Spike & \\
\hline & $\mathrm{Na}$ & $\mathrm{K}$ & $\mathrm{Cs}^{* *}$ & $\mathrm{Na}$ & $\mathrm{K}$ & $\mathrm{Cs}^{* *}$ & $\mathrm{Na}$ & $\mathrm{K}$ & $\mathrm{Cs}^{* *}$ & $\mathrm{Na}$ & $\mathrm{K}$ & $\mathrm{Cs}^{* *}$ & $\mathrm{Na}$ & $\mathrm{K}$ & $\mathrm{Cs}^{* *}$ & $\mathrm{Na}$ & $\mathrm{K}$ & $\mathrm{Cs}^{* *}$ \\
\hline Scrub 1 & $7.4(2)$ & $5.72(6)$ & 0.17 & $6.4(3)$ & $13.9(3)$ & 0.13 & $5.6(1)$ & $23.3(1)$ & 0.03 & $8.9(1)$ & $6.43(6)$ & N/A & $7.93(6)$ & $5.39(2)$ & 0.12 & $8.0(1)$ & $0.020(5)$ & 0.18 \\
\hline Scrub 2 & $0.33(6)$ & $0.57(6)$ & 0.19 & $0.42(6)$ & $1.26(8)$ & 0.19 & $0.37(3)$ & $2.19(1)$ & 0.02 & $0.3(1)$ & $0.63(1)$ & $\mathrm{N} / \mathrm{A}$ & $0.38(5)$ & $0.70(3)$ & 0.19 & $0.14(9)$ & $0.017(5)$ & 0.15 \\
\hline \multicolumn{19}{|l|}{ Scrub } \\
\hline Total & $7.7(2)$ & $6.29(7)$ & 0.36 & $6.8(4)$ & $15.2(3)$ & 0.32 & $6.0(1)$ & $25.5(1)$ & 0.05 & $9.2(2)$ & $7.06(7)$ & N/A & $8.3(1)$ & $6.09(5)$ & 0.31 & $8.2(2)$ & $0.037(9)$ & 0.33 \\
\hline Strip 1 & $<0.01$ & $<0.01$ & 0.17 & $<0.01$ & $0.036(4)$ & 0.20 & $<0.01$ & $0.057(5)$ & 0.45 & $<0.01$ & $0.029(5)$ & N/A & $0.013(4)$ & $0.141(6)$ & 0.21 & $0.015(5)$ & $0.070(4)$ & 0.23 \\
\hline Strip 2 & $<0.01$ & $<0.01$ & $<0.01$ & $<0.01$ & $<0.01$ & $<0.01$ & $<0.01$ & $<0.01$ & 0.03 & $<0.01$ & $<0.01$ & N/A & $<0.01$ & $<0.01$ & $<0.01$ & $<0.01$ & $<0.01$ & $<0.01$ \\
\hline Strip 3 & $<0.01$ & $<0.01$ & $<0.01$ & $<0.01$ & $<0.01$ & $<0.01$ & $<0.01$ & $<0.01$ & $<0.01$ & $<0.01$ & $<0.01$ & N/A & $<0.01$ & $<0.01$ & $<0.01$ & $<0.01$ & $<0.01$ & $<0.01$ \\
\hline \multicolumn{19}{|l|}{ Strip } \\
\hline total & $<0.01$ & $<0.01$ & 0.17 & $<0.01$ & $0.036(4)$ & 0.2 & $<0.01$ & $0.057(5)$ & 0.48 & $<0.01$ & $0.029(5)$ & N/A & $0.013(4)$ & $0.141(6)$ & 0.21 & $0.015(5)$ & $0.070(4)$ & 0.23 \\
\hline $\begin{array}{l}\text { Total } \\
{[\mathrm{mM}]}\end{array}$ & & $14.5(3)$ & & & $22.5(7)$ & & & $32.0(2)$ & & & $16.3(3)$ & & & $15.1(1)$ & & & $8.9(2)$ & \\
\hline
\end{tabular}
both the scrub and strip stages.

**Cesium concentrations determined using the distribution ratios for each of the different simulants as shown in Table based on an initial $[\mathrm{Cs}]_{\mathrm{Aq}}$ of $0.14 \mathrm{mM}$. 


\subsection{EMULSION FORMATION}

A qualitative test gave a good idea of the conditions under which the emulsion could form. The danger of emulsion formation in the NG-CSSX system is negligible; with the current boric acid concentration of $0.01 \mathrm{M}$, the DCiTG suppressor concentration could be increased ten-fold (from 0.003 to $0.03 \mathrm{M}$ ) before any adverse effects would be seen. Emulsion formation has been visually observed when the boric acid concentration was $>0.1 \mathrm{M}$ or when the DCiTG suppressor concentration was $>0.03 \mathrm{M}$ (see Fig. 8.2).

Fig. 8.2 shows more clearly the qualitative limits of DCiTG and boric acid concentrations that form an emulsion after three consecutive contacts with boric acid by using a log scale of the boric acid concentration.
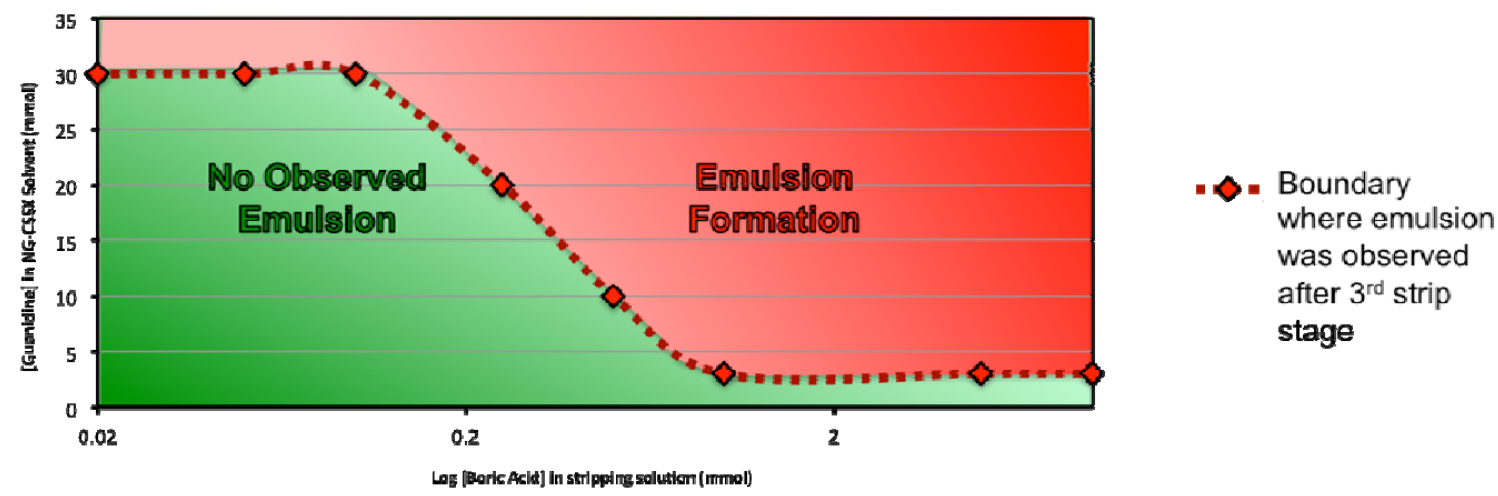

Fig. 8.2. Emulsion boundary formation as a function of guanidine and boric acid concentrations

\subsection{INTERFACIAL TENSION}

An increase in the DCiTG in the NG-CSSX solvent and the boric acid in the strip solution resulted in a dramatic reduction of the interfacial tension of the stripping stage.

Interfacial tension measurements have shown that an increased DCiTG concentration in the NG-CSSX solvent results in a dramatic reduction in the interfacial tension, even when no boric acid is present in the strip solution (see Table 8.10). This reduction is, however, increased with increasing boric acid concentration. The contacts where the interfacial tension was below the detectable limit of the instrument $\left(<1\right.$ dyne $\left.\mathrm{cm}^{-1}\right)$ correspond well with the formation of an emulsion as described above. 
Table 8.10. Measured interfacial tensions

\begin{tabular}{lllc}
\hline \multicolumn{1}{c}{ Organic Phase } & [DCiTG] & Aqueous Phase & Interfacial Tension ${\left.\mathbf{~ d y n e ~} \mathbf{~ c m}^{-1}\right)}^{-}$ \\
\hline Isopar L & - & $\mathrm{H}_{2} \mathrm{O}$ & $47.5(6)$ \\
Isopar L & - & $\mathrm{H}_{3} \mathrm{BO}_{3}(0.01 \mathrm{M})$ & $45.9(7)$ \\
Isopar L & - & $\mathrm{H}_{3} \mathrm{BO}_{3}(0.05 \mathrm{M})$ & $45.4(5)$ \\
NG-CSSX solvent & $0.003 \mathrm{M}$ & $\mathrm{H}_{2} \mathrm{O}$ & $13.8(4)$ \\
NG-CSSX solvent & $0.030 \mathrm{M}$ & $\mathrm{H}_{2} \mathrm{O}$ & $4.9(2)$ \\
NG-CSSX solvent & $0 \mathrm{M}$ & $\mathrm{H}_{3} \mathrm{BO}_{3}(0.01 \mathrm{M})$ & $16.7(9)$ \\
NG-CSSX solvent & $0.003 \mathrm{M}$ & $\mathrm{H}_{3} \mathrm{BO}_{3}(0.01 \mathrm{M})$ & $8.8(5)$ \\
NG-CSSX solvent & $0.015 \mathrm{M}$ & $\mathrm{H}_{3} \mathrm{BO}_{3}(0.01 \mathrm{M})$ & $1.7(4)$ \\
NG-CSSX solvent & $0.030 \mathrm{M}$ & $\mathrm{H}_{3} \mathrm{BO}_{3}(0.01 \mathrm{M})$ & $<1$ \\
NG-CSSX solvent & $0 \mathrm{M}$ & $\mathrm{H}_{3} \mathrm{BO}_{3}(0.05 \mathrm{M})$ & $15.5(3)$ \\
NG-CSSX solvent & $0.003 \mathrm{M}$ & $\mathrm{H}_{3} \mathrm{BO}_{3}(0.05 \mathrm{M})$ & $7.2(3)$ \\
NG-CSSX solvent & $0.015 \mathrm{M}$ & $\mathrm{H}_{3} \mathrm{BO}_{3}(0.05 \mathrm{M})$ & $<1$ \\
NG-CSSX solvent & $0.030 \mathrm{M}$ & $\mathrm{H}_{3} \mathrm{BO}_{3}(0.05 \mathrm{M})$ & $<1$ \\
\hline
\end{tabular}

The interfacial tension for the optimized stripping conditions for the NG-CSSX process (i.e., 0.003 M DCiTG and $0.01 \mathrm{M}$ Boric acid) was only $5 \mathrm{dyne}^{-1}$ less than the NG-CSSX solvent (0.003 M DCiTG) in contact with deionized water and so would not affect the process.

\subsection{BREAKING THE EMULSION}

It was shown that even in an extreme case, where the DCiTG concentration was $0.02 \mathrm{M}$ and the boric acid concentration was $0.1 \mathrm{M}$, increasing the ionic strength of the strip solution or introduction of a more lipophilic anion (e.g., $\mathrm{SCN}^{-}$) by the addition of a simple salt, will quickly break any emulsion formed (see Table 8.11).

Washing a preformed emulsion with water was shown to have no effect. The emulsion can be broken if contacted repeatedly with mineral acids $\left(\mathrm{HCl} / \mathrm{HNO}_{3}\right)$, but the introduction of salts of more lipophilic anions (NaSCN, NaOTf) was shown to be more efficient. These may, however, result in a reduction of the $D_{C s}$ value for stripping, requiring several cycles before complete removal from the NG-CSSX system.

Table 8.11. Attempts at "breaking" the emulsion formed between the NG-CSSX Solvent and boric acid

\begin{tabular}{|c|c|c|c|c|}
\hline Solution & Conc. & $\mathbf{O}: \mathbf{A}$ & Observation & Comments \\
\hline $\mathrm{H}_{2} \mathrm{O}$ & - & $3.75: 1$ & Unchanged & - \\
\hline $\mathrm{H}_{2} \mathrm{O}$ & - & $3.75: 2$ & Unchanged & - \\
\hline $\mathrm{H}_{2} \mathrm{O}$ & - & $3.75: 4$ & Unchanged & - \\
\hline $\mathrm{HCl}$ & $0.05 \mathrm{M}$ & $3.75: 1$ & $\begin{array}{l}\text { Emulsion partially } \\
\text { broken } \\
\text { Emulsion broken }\end{array}$ & $\begin{array}{l}\text { Further broken upon second } \\
\text { contact }\end{array}$ \\
\hline $\mathrm{NaCl}$ & $0.1 \mathrm{M}$ & $3.75: 1$ & $\begin{array}{l}\text { upon vortexing } \\
\text { Emulsion partially }\end{array}$ & - \\
\hline $\begin{array}{l}\mathrm{HNO}_{3} \\
\mathrm{HNa}_{2} \mathrm{CO}_{3}\end{array}$ & $\begin{array}{l}0.05 \mathrm{M} \\
0.1 \mathrm{M}\end{array}$ & $\begin{array}{l}3.75: 1 \\
3.75: 1\end{array}$ & $\begin{array}{l}\text { broken } \\
\text { Emulsion broken }\end{array}$ & second contact \\
\hline
\end{tabular}


Table 8.11. Attempts at "breaking" the emulsion formed between the NG-CSSX Solvent and boric acid

\begin{tabular}{|c|c|c|c|c|}
\hline Solution & Conc. & $\mathbf{O}: \mathbf{A}$ & Observation & Comments \\
\hline $\mathrm{NaSCN}$ & $0.1 \mathrm{M}$ & $3.75: 1$ & Emulsion broken & $\begin{array}{l}\text { Phase separation } \\
\text { instantaneous } \\
\text { Phase separation }\end{array}$ \\
\hline $\mathrm{NaOTf}$ & $0.1 \mathrm{M}$ & $3.75: 1$ & $\begin{array}{l}\text { Emulsion broken } \\
\text { Emulsion partially }\end{array}$ & $\begin{array}{l}\text { instantaneous } \\
\text { Emulsion broken upon }\end{array}$ \\
\hline $\mathrm{NH}_{3}$ & $28 \%$ in $\mathrm{H}_{2} \mathrm{O}$ & $3.75: 1$ & broken & vortexing \\
\hline Sodium dodecyl Sulfate (SDS) & $1 \times 10^{-6} \mathrm{M}$ & $3.75: 1$ & Unchanged & - \\
\hline ISOPAR L & - & $3.75: 1^{*}$ & Emulsion thickens & Remains thick after 1 hour \\
\hline ISOPAR L & - & $3.75: 2 *$ & Emulsion thickens & Releases solvent after 1 hour \\
\hline ISOPAR L & - & $3.75: 4^{*}$ & Emulsion thickens & Releases solvent after 1 hour \\
\hline
\end{tabular}

*NG-CSSX solvent : Organic diluent

\subsection{CONCLUSIONS AND RECOMMENDATIONS}

The temperature dependence of the NG-CSSX process is considerably stronger than the baseline BOBCalixC6 CSSX process currently in use. It is possible to linearize the data according to the Van't Hoff formalism as an aid for interpolation, and the regression parameters are provided. Curvature in the extraction and scrubbing plots of $\log D_{\mathrm{Cs}}$ versus $1000 / T$ can be easily explained by potassium loading. It follows that the temperature dependence should be revisited if other feed types are considered, especially for feeds containing higher potassium levels. Taking advantage of the temperature control in the MCU, 23 $\pm 3{ }^{\circ} \mathrm{C}$ on extraction and $33 \pm 3{ }^{\circ} \mathrm{C}$ on stripping, the stronger dependence on temperature in the NG-CSSX process versus the baseline BOBCalixC6 CSSX process means an even greater benefit in terms of decontamination in the limited number of stages of the MCU.

As shown in Table 8.5, NG-CSSX solvent extraction chemistry has the ability to continue performing effectively in the presence of increased concentrations of sodium, at least when all solution components change in proportion to the sodium concentration. These results, albeit encouraging, must be followed by further testing of an increased waste-processing rate from employing more concentrated salt waste feed solutions. Continued study of NG-CSSX as well as system-wide operations are needed to evaluate the potential benefit.

Assuming that contactor performance is not negatively impacted, a modular flowsheet would perform well at any of the sodium concentrations tested, based on flowsheet calculations conducted previously $3,8,10$ For the fixed configuration of the SRS Modular BOBCalixC6 CSSX Unit, consisting of seven extraction, two scrubs, seven strips, and one wash stage ${ }^{20,24,31}$, a target DF of 40,000 should be attainable at or above 5.6 M, subject to reasonable expectations regarding stage efficiency and other-phase carryover. It is less certain that a strict DF goal of 40,000 would be met in the MCU configuration at sodium concentrations 
lower than 5.6 M, although a DF of 40,000 may not be needed to meet SRS waste-acceptance criteria for the decontaminated salt stream ${ }^{3}$. ICP analysis of the aqueous feeds for alkaline metals has further confirmed the efficacy of the $\mathrm{NaOH}$ scrubbing stages for successful removal of potassium and sodium. The introduction of lipophilic impurities, however, does impede the scrubbing stages such that the results is a retention of potassium.

Given that a substantial increase in waste-processing rate is in principle possible based upon increased salt waste concentration in processing, R\&D activities can be identified toward a systems level evaluation. Below is an initial short list of potential R\&D activities:

1. Evaluate the aqueous feed envelope expected from retrieval. Define average and bounding compositions and representative waste simulants for testing. Identify real waste with elevated sodium levels for testing. Examine potential effects related to aluminosilicate precipitation.

2. Examine impacts to actinide/plutonium removal efficiency and processing rate, including filtration.

3. Examine impacts in NG-CSSX processing, including batch tests with simulants and real waste, contactor hydraulics, stage efficiency, and coalescer performance.

4. Examine impacts to salt-stone processing. 


\section{KINETICS OF CESIUM EXTRACTION AND STRIPPING}

Data obtained in this work suggests that the extraction and stripping kinetics of the NG-CSSX process are significantly faster than the BOBCalixC6 CSSX process currently in use at SRS. Given that the NGCSSX process employs a different solvent and a different stripping chemistry as compared with the BOBCalixC6 CSSX process, it was necessary to ensure that the kinetics of NG-CSSX are at least as fast as that of BOBCalixC6 CSSX. A series of timed contacts of the NG-CSSX solvent with simulated SRS salt waste were conducted by a vortexing technique for times up to $60 \mathrm{~s}$. Cesium distribution was determined at each time by gamma counting using ${ }^{137} \mathrm{Cs}$ tracer. For the stripping tests, a truncated form of a batch ESS experiment was employed using SRS waste simulant as the feed, $50 \mathrm{mM} \mathrm{NaOH}$ as the scrub solution, and $10 \mathrm{mM}$ boric acid as the strip solution. Following two scrub contacts, the kinetics of stripping were determined in a manner identical to that of the extraction tests. Kinetics of NG-CSSX surpassed that of BOBCalixC6 CSSX in both extraction and stripping, 90\% mass transfer being reached at $7 \mathrm{~s}$ and $9 \mathrm{~s}$, respectively, less than a half and a third of the corresponding times for BOBCalixC6 CSSX solvent.

\subsection{INTRODUCTION}

Under funding from the DOE-EM Office of Technology Innovation and Development, recent laboratory efforts have been directed toward developing the NG-CSSX process for cesium removal from alkaline tank wastes ${ }^{1}$. In support of EM's drive to accelerate processing of tank wastes at the SRS and Hanford $\operatorname{site}^{2}$, the laboratory efforts have sought increased performance of NG-CSSX, both in decontamination efficiency and waste-processing rate ${ }^{3}$. A key to realizing these dual goals lies in the kinetic performance of NG-CSSX being at least as fast as the BOBCalixC6 CSSX process currently implemented at the SRS. With improved kinetics, mass-transfer efficiencies would be expected to improve, leading to higher DFs, higher throughputs as limited by hydraulics rather than mass transfer, and greater flexibility in contactor operation. High mass-transfer efficiencies are especially important for NG-CSSX, because the benefit of the very high and low $D_{\mathrm{Cs}}$ respectively attained by NG-CSSX chemistry in extraction and stripping can only be realized if a very high fraction of the theoretical mass transfer is actually delivered in the brief residence time of the phases in the mixing zone of the contactor. Flowsheet calculations reported recently for NG-CSSX assumed $>90 \%$ stage efficiencies to exceed a target DF of $40,000^{3}$.

The technical objective of this report is to compare the kinetics of extraction and stripping using the NGCSSX and BOBCalixC6 CSSX solvents in simple vortexing contacting experiments. Prior kinetics data in early chemical development obtained by hand vortexing showed that $90 \%$ stripping is reached at 
approximately $10 \mathrm{~s}$, suggesting reasonable mass-transfer efficiencies would be achievable in centrifugal contactors $^{26}$. Subsequently, conditions were identified for achieving acceptable stage efficiencies in engineering tests of the BOBCalixC6 CSSX process using intermediate- $(5 \mathrm{~cm})^{27},{ }^{28},{ }^{29}$ and full-size $(25.4$ $\mathrm{cm})^{30}$ centrifugal contactors. However, stage efficiencies were shown to be sensitive to contactor design features (e.g., curved versus straight vanes in the bottom of the contactor housing) and operating conditions, leading in certain tests to stage efficiencies falling below the $80 \%$ target used in flowsheet calculations. Stripping has tended to be more problematic than extraction. Despite such limitations, the MCU has greatly exceeded its design DF of 12, achieving sustained operation at DFs exceeding $200^{31}$. Stage efficiencies in the full-scale BOBCalixC6 CSSX process have thus proven adequate in practice. Taking results of simple liquid Liquid vortexing experiments as a qualitative indicator of potential kinetic problems, a demonstration of faster kinetics for NG-CSSX in comparison with BOBCalixC6 CSSX is expected to provide confidence that there should be no kinetic limitations in the implementation of the new solvent system or in the interpretation of future contactor stage-efficiency results.

\subsection{EXPERIMENTAL SECTION}

\subsubsection{Materials}

\subsubsection{Solvent Components}

Solvent components were obtained from commercial sources and judged to be of adequate purity for use as received. $N, N^{\prime}$-Dicyclohexyl- $N^{\prime \prime}$-isotridecylguanidine, the active guanidine reagent in LIX $^{\circledR} 79$, was supplied by Cognis. Calix[4]arene-bis(tert-octylbenzocrown-6) (BOBCalixC6) was obtained from IBC Advanced Technologies. 1-(2,2,3,3-Tetrafluoropropoxy),-3-[4-(sec-butyl)phenoxy]-2-propanol (Cs-7SB modifier) and MaxCalix were obtained from Marshallton Research. Tri- $n$-octylamine (TOA) was obtained from Aldrich, and Isopar $^{\circledR} \mathrm{L}$ was obtained from ExxonMobil. Solvents were prepared by weighing appropriate amounts of extractant, modifier, and suppressor (TOA or LIX 79 guanidine) into volumetric flasks and diluting with Isopar L to the mark. The components are shown in Table 9.1.

Table 9.1. BOBCalixC6 CSSX and NG-CSSX solvent components

\begin{tabular}{lll}
\hline Component & \multicolumn{1}{c}{ Code } & Chemical Name \\
\hline Extractant & MaxCalix & $\begin{array}{l}\text { 1,3-alt-25,27-bis(3,7- } \\
\text { dimethyloctyl-1-oxy) } \\
\text { calix[4]arene- } \\
\text { benzocrown-6 }\end{array}$
\end{tabular}


Table 9.1. BOBCalixC6 CSSX and NG-CSSX solvent components

\begin{tabular}{|c|c|c|c|}
\hline Component & Code & Chemical Name & Structure \\
\hline Extractant & BOBCalixC6 & $\begin{array}{l}\text { Calix[4]arene-bis(tert- } \\
\text { octylbenzocrown-6) }\end{array}$ & \\
\hline Modifier & Cs-7SB & $\begin{array}{l}\text { 1-(2,2,3,3- } \\
\text { Tetrafluoropropoxy)-3- } \\
\text { (4-sec-butylphenoxy)- } \\
\text { 2-propanol }\end{array}$ & \\
\hline Suppressor & LIX 79 & $\begin{array}{l}N, N^{\prime} \text {-Dicyclohexyl- } N " \text { - } \\
\text { isotridecylguanidine }\end{array}$ & \\
\hline Suppressor & TOA & Tri- $n$-octylamine & \\
\hline Diluent & Isopar L & $\begin{array}{l}\mathrm{C}_{12} \text {-isoparaffinic } \\
\text { hydrocarbon }\end{array}$ & \\
\hline
\end{tabular}

\subsubsection{Waste Tank Simulant Composition}

The targeted composition of the SRS-15 tank-waste simulant used in the extraction contacts is given in Table 9.2 according to the preparative method described in detail previously ${ }^{7}$. The SRS- 15 simulant is designed to represent the average SRS tank-waste composition ${ }^{9}$.

Table 9.2. Target composition of aqueous tankwaste simulant SRS-15

\begin{tabular}{ll}
\hline \multicolumn{1}{c}{ Analyte or Species } & mol/L \\
\hline Principal constituents: & \\
$\mathrm{Al}$ as $\left(\mathrm{Al}(\mathrm{OH})_{4}{ }^{-}\right)$ & 0.280 \\
$\mathrm{Cl}^{-}$ & $2.4 \times 10^{-2}$ \\
$\mathrm{CO}_{3}{ }^{2-}$ (TIC) & 0.150 \\
$\mathrm{Cs}^{+}$(Total) & $1.4 \times 10^{-4}$ \\
$\mathrm{~K}^{+}$ & 0.015 \\
$\mathrm{Na}^{+}$ & 5.60 \\
$\mathrm{NO}_{2}{ }^{-}$ & 0.500 \\
$\mathrm{NO}_{3}^{-}$ & 2.03 \\
$\mathrm{OH}^{-}$(Free) & 2.06 \\
$\mathrm{SO}_{4}{ }^{2-}$ & 0.140 \\
\hline $\mathrm{Minor}$ inorganic constituents: & \\
$\mathrm{Ag}(\mathrm{I})$ & $9.3 \times 10^{-8}$ \\
$\mathrm{CrO}$ & ${ }_{4}^{2-}$ \\
$\mathrm{Cu}(\mathrm{II})$ & $1.4 \times 10^{-3}$ \\
& $2.3 \times 10^{-5}$
\end{tabular}


Table 9.2. Target composition of aqueous tankwaste simulant SRS-15

\begin{tabular}{lc}
\hline \multicolumn{1}{c}{ Analyte or Species } & mol/L \\
\hline $\mathrm{F}^{-}$ & $2.8 \times 10^{-2}$ \\
$\mathrm{Fe}(\mathrm{III})$ & $2.6 \times 10^{-5}$ \\
$\mathrm{Hg}(\mathrm{II})$ & $2.5 \times 10^{-7}$ \\
$\mathrm{MoO}_{4}{ }^{2-}$ & $7.0 \times 10^{-5}$ \\
$\mathrm{NH}_{3}$ & $1.0 \times 10^{-3}$ \\
$\mathrm{~Pb}(\mathrm{II})$ & $1.0 \times 10^{-5}$ \\
$\mathrm{Pd}(\mathrm{II})$ & $3.8 \times 10^{-6}$ \\
$\mathrm{PO}_{4}{ }^{3-}$ & $7.0 \times 10^{-3}$ \\
$\left.\mathrm{Rh}^{-} \mathrm{III}\right)$ & $2.0 \times 10^{-6}$ \\
$\mathrm{Ru}(\mathrm{III})$ & $8.1 \times 10^{-6}$ \\
$\mathrm{Si}(\mathrm{IV})$ & $3.0 \times 10^{-2}$ \\
$\mathrm{Sn}(\mathrm{II})$ & $2.0 \times 10^{-5}$ \\
\hline Minor organic constituents: & \\
$n$-Butanol & $2.7 \times 10^{-5}$ \\
Tri- $n$-butylphosphate (TBP) & $1.9 \times 10^{-6}$ \\
Di- $n$-butylphosphate (DBP) & $1.2 \times 10^{-4}$ \\
Mono- $n$-butylphosphate (MBP) & $1.6 \times 10^{-4}$ \\
Formate $\left(\mathrm{HCO}_{2}{ }^{-}\right)$ & $3.3 \times 10^{-2}$ \\
Oxalate $\left(\mathrm{C}_{2} \mathrm{O}_{4}{ }^{2-}\right)$ & $8.0 \times 10^{-3}$ \\
Trimethylamine & $1.7 \times 10^{-4}$ \\
\hline
\end{tabular}

\subsubsection{Methods}

\subsubsection{General}

Multiple solvents comprised of MaxCalix or BOBCalixC6, Cs-7SB modifier, and LIX 79 guanidine or TOA in Isopar L were used during the kinetics experiments. The solvents were prepared and then prewashed prior to use in the following manner: one contact sequentially with $0.010 \mathrm{M} \mathrm{HCl}, \mathrm{H}_{2} \mathrm{O}$, then decreasing concentrations of $\mathrm{NaOH}(0.3 \mathrm{M}, 0.1 \mathrm{M}, 0.03 \mathrm{M}$, and $0.01 \mathrm{M})$, and then once or twice with $\mathrm{H}_{2} \mathrm{O}$ until the solution was $\mathrm{pH}$ neutral. The compositions of the solvents are given in Table 9.3. 
Table 9.3. Solvent compositions used in kinetics experiments ${ }^{a}$

\begin{tabular}{ccccc}
\hline & & Modified & Modified & \\
Solvents & CSSX & NG-CSSX (A) & NG-CSSX (B) & NG-CSSX \\
\hline Extractant & BOBCalixC6 $(0.007 \mathrm{M})$ & MaxCalix $(0.007 \mathrm{M})$ & MaxCalix $(0.007 \mathrm{M})$ & MaxCalix $(0.05 \mathrm{M})$ \\
Modifier & Cs-7SB $(0.75 \mathrm{M})$ & Cs-7SB $(0.75 \mathrm{M})$ & Cs-7SB $(0.5 \mathrm{M})$ & Cs-7SB $(0.5 \mathrm{M})$ \\
Suppressor & TOA $(0.003 \mathrm{M})$ & LIX 79 $(0.003 \mathrm{M})$ & LIX 79 $(0.003 \mathrm{M})$ & LIX 79 $(0.003 \mathrm{M})$ \\
\hline
\end{tabular}

${ }^{a}$ The components are dissolved in Isopar L diluent, an isoparafinic hydrocarbon.

\subsubsection{Cesium Distribution Ratios}

$D_{\mathrm{Cs}}$ for the extraction kinetics for all four solvents with SRS-15 simulant were obtained by contacting aqueous and organic phases vortexing using a Vortex-Genie 1 Touch Mixer with an organic to O:A of 1:4. Phases were contacted in $15 \mathrm{~mL}$ polypropylene centrifuge tubes for varying lengths of time. Prior to contacting, a spike of ${ }^{137} \mathrm{Cs}$ was added to the SRS-15 simulant and the solution vortexed for $30 \mathrm{~s}$ and centrifuged so that the spike was distributed throughout the simulant. The organic phase was then carefully pipetted down the inside tube wall, ensuring the phases would not mix prior to contacting. The liquid phases were vortexed at ambient temperature $\left(22-25^{\circ} \mathrm{C}\right)$. Immediately thereafter, the tubes were centrifuged for $1 \mathrm{~min}$. at $3000 \mathrm{RPM}$ at ambient temperature. An appropriate aliquot of each phase was subsampled and counted using a Packard Cobra II Auto-Gamma counter. $D_{\mathrm{Cs}}$ are given as the ratio of the background-corrected volumetric count rates of the radioisotope in each phase at equilibrium.

\subsubsection{Stripping Kinetics of the NG-CSSX Solvent}

The stripping kinetics of NG-CSSX solvent with boric acid were obtained by contacting the phases in a manner similar to that used for determining the kinetics of extraction. The initial extraction and scrub contacts were performed as described previously ${ }^{3}$, with $\mathrm{ES}_{2} \mathrm{~S}_{3}(\mathrm{O}: \mathrm{A}=1: 4$ for extraction and 3.75:1 for scrubbing). Phases were contacted in polypropylene micro-tubes mounted by clips on a disk rotated for 60 minutes for extractions and 45 minutes for scrubs and strips. The solutions were contacted inside an airbox maintained at a temperature of $25 \pm 0.2{ }^{\circ} \mathrm{C}$. After the contacting period, the tubes were centrifuged for $5 \mathrm{~min}$. at $3000 \mathrm{RPM}$ at the experimental temperature. After the second scrub, an aliquot of the organic phase was subsampled and counted using a Packard Cobra II Auto-Gamma counter. The NG-CSSX solvent that had gone through the extraction and scrubbing stages was then used in the strip kinetics tests. Prior to addition of this prepared solvent, a set amount of $0.010 \mathrm{M} \mathrm{H}_{3} \mathrm{BO}_{3}$ was placed into a $15 \mathrm{~mL}$ polypropylene centrifuge tube and the solvent added gently as described for the extraction test. The tubes were then hand-vortexed for a set length of time and centrifuged for $1 \mathrm{~min}$. at $3000 \mathrm{RPM}$ at ambient temperature. An appropriate aliquot of each phase was subsampled and counted. $D_{\mathrm{Cs}}$ are given as the ratio 
of the background-corrected volumetric count rates of the radioisotope in each phase at equilibrium.

Stripping kinetics of the current BOBCalixC6 CSSX solvent in use at the SRS were determined to allow for a direct comparison to the NG-CSSX solvent. The method used to determine stripping kinetics of the BOBCalixC6 CSSX solvent was similar to that utilized for the NG-CSSX stripping kinetics, except that the scrub and strip aqueous phases appropriate for the BOBCalixC6 CSSX flowsheet ${ }^{7,32,33}$, were employed. Accordingly, in place of $0.025 \mathrm{M} \mathrm{NaOH}$ for the scrub solution, a solution of $0.050 \mathrm{M} \mathrm{HNO}_{3}$ was used, and $0.001 \mathrm{M} \mathrm{HNO}_{3}$ was used in place of $0.010 \mathrm{M} \mathrm{H}_{3} \mathrm{BO}_{3}$. For experimental consistency, however, the NG-CSSX phase ratios were retained in all tests: $\mathrm{O}: \mathrm{A}=1: 4$ for extraction and $\mathrm{O}: \mathrm{A}=3.75: 1$ for scrub and strip (CSSX uses O:A = 1:3 and 5:1 correspondingly).

Kinetic efficiencies (fractional distribution) were calculating using the Murphree definition based on the background-corrected aqueous-phase tracer counts for extraction and organic-phase counts for stripping. Murphree efficiency $E$ can be calculated using Eq. 9.127.

$$
E=\frac{\left(\left(\frac{A_{t}}{A_{o}}\right)-1\right)}{\left(\left(\frac{A_{f}}{A_{o}}\right)-1\right)}
$$

In Eq. 9.1, $A_{\mathrm{t}}$ represents the concentration of cesium in the source phase at a specific time, $A_{\mathrm{o}}$ is the initial concentration in the source phase, and $A_{\mathrm{f}}$ is the final concentration. The value of $A_{\mathrm{f}}$ was difficult to determine exactly, owing to slight drift in the value of $D_{C s}$ for extended contacts to $60 \mathrm{~s}$, presumably due to the slight temperature drift over the time interval. To correct for the points where this was observed, the later three points were taken and averaged to give a value assumed to be the final concentration.

\subsection{RESULTS AND DISCUSSION}

\subsubsection{Kinetics of Extraction of MaxCalix and BobCalix C6}

The time dependent extraction of cesium measured as $D_{C S}$ from SRS simulated waste by BOBCalixC6 CSSX, NG-CSSX, and modified NG-CSSX solvents is given in Table 9.4. In Fig. 9.1, the extraction of cesium by multiple solvents is expressed as the fractional distribution over time (Murphree efficiency). From the curves seen in Fig. 9.1, it can be observed that the NG-CSSX (50 mM MaxCalix) solvent performs much better than the $7 \mathrm{mM}$ BOBCalixC6 CSSX solvent currently in use at SRS. Time to reach $90 \%$ mass transfer is only $7 \mathrm{~s}$ for NG-CSSX versus $16 \mathrm{~s}$ for BOBCalixC6 CSSX. The difference arises 
from the high calixarene concentration of the NG-CSSX solvent. Mono- and biscrown calix[4]arenes in general have approximately the same extraction strength for cesium ${ }^{34}$ and presumably similar kinetics, which is reflected in the overlap of the BOBCalixC6 CSSX and modified NG-CSSX (A) curves. Furthermore, the concentration of modifier appears to have no direct effect on the kinetics of the solvent during extraction, as shown by the overlap of the two $7 \mathrm{mM}$ MaxCalix curves. Thus, in addition to higher $D_{C s}$ on extraction, a benefit of the higher concentration of calixarene in the NG-CSSX solvent is much faster extraction kinetics.

\subsubsection{Kinetics of Stripping of MaxCalix and BOBCalixC6}

The stripping kinetics for BOBCalixC6 CSSX, NG-CSSX, and modified NG-CSSX solvents are given in Table 9.5 and Fig. 9.2. Again, it can be observed that NG-CSSX stripping is much faster than that for BOBCalixC6 CSSX. However, in this case, the difference is ascribable to the different stripping chemistries employed. Time to reach $90 \%$ stripping is $38 \mathrm{~s}$ for BOBCalixC6 CSSX and $9 \mathrm{~s}$ for both the NG-CSSX and modified NG-CSSX (B) solvents. The concentration of MaxCalix is apparently only a minor effect. It may be seen that the $7 \mathrm{mM}$ MaxCalix solvent strips just slightly faster, which may be real in that the higher MaxCalix concentration would raise the $D_{\mathrm{Cs}}$ value.

Table 9.4. $D_{\mathrm{Cs}}$ for extraction by BOBCalixC6 CSSX, NG-CSSX, and modified NG-CSSX solvents as a function of time ${ }^{a}$

\begin{tabular}{|c|c|c|c|c|}
\hline \multirow[t]{2}{*}{ Time (seconds) } & \multicolumn{4}{|c|}{$D_{\mathrm{Cs}}$} \\
\hline & $\begin{array}{c}\text { CSSX } \\
(7 \mathrm{mM} \text { BOBCalix })\end{array}$ & $\begin{array}{c}\text { Modified } \\
\text { NG-CSSX (A) } \\
\text { (7 mM MaxCalix) }\end{array}$ & $\begin{array}{c}\text { Modified } \\
\text { NG-CSSX (B) } \\
\text { (7 mM MaxCalix) }\end{array}$ & $\begin{array}{c}\text { NG-CSSX } \\
\text { (50 mM MaxCalix) }\end{array}$ \\
\hline 5 & 3.9 & 2.9 & 3.2 & 19.0 \\
\hline 10 & 5.7 & 6.0 & 5.6 & 34 \\
\hline 15 & 9.2 & 8.1 & 7.3 & 44 \\
\hline 20 & 10.8 & 10.8 & 10.1 & 53 \\
\hline 30 & 12.8 & 12.5 & 13 & 55.9 \\
\hline 45 & 15.3 & 15 & 14.2 & 58.0 \\
\hline 60 & 14.4 & 11.5 & 14.6 & 56.1 \\
\hline
\end{tabular}

\footnotetext{
${ }^{a}$ The solvent compositions are given in Table 9.3. The aqueous phase for extraction was SRS-15 waste simulant, whose
} components are given in Table 9.2. The O:A ratios for extraction contacts were 1:4, and all contacts were run at $22-25^{\circ} \mathrm{C}$. 


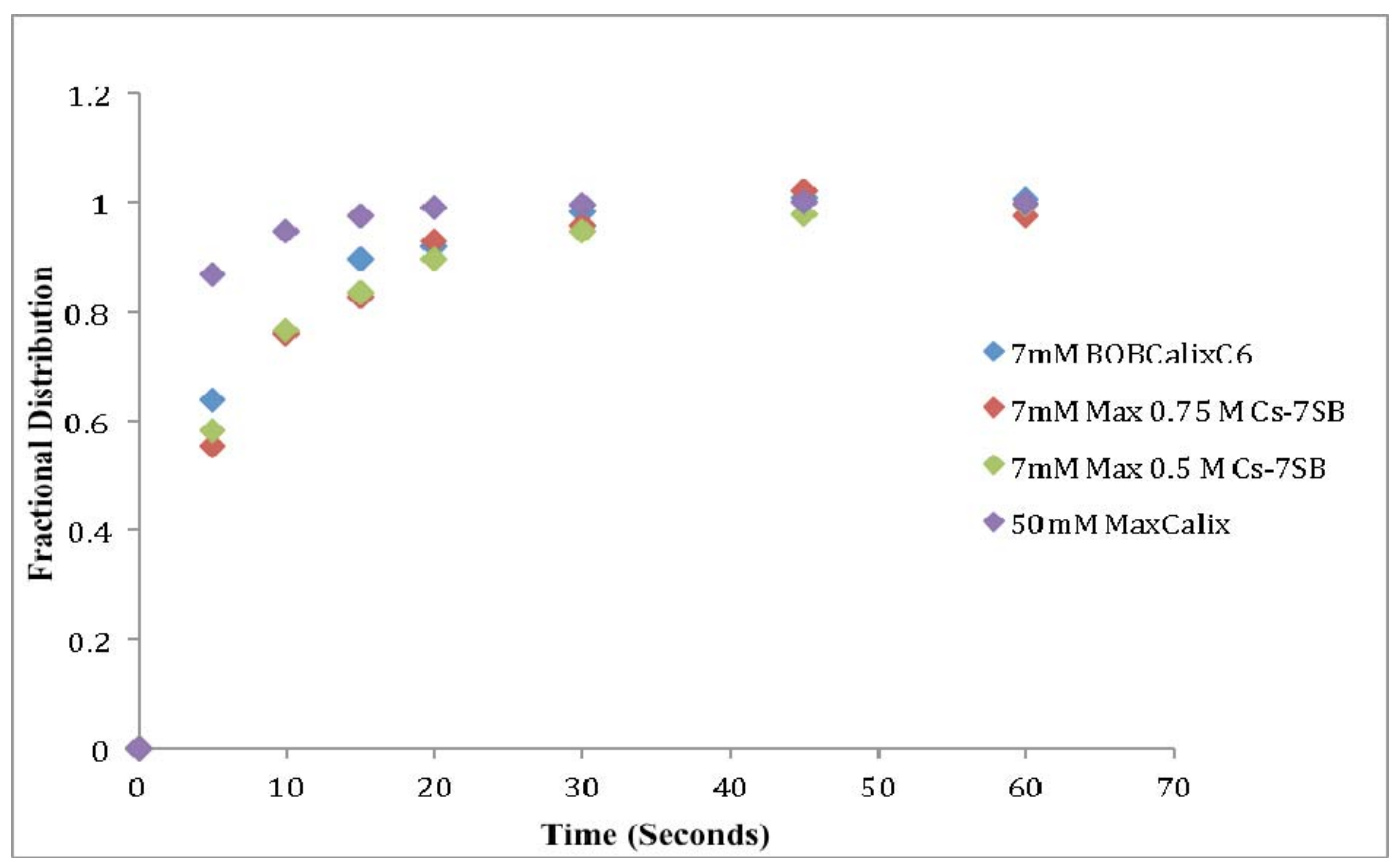

Fig. 9.1. Plot of fractional distribution versus time for extraction. Fractional distribution (Murphree efficiency) of NG-CSSX (50 mM MaxCalix), BOBCalixC6 CSSX (7 mM BOBCalix), modified NB-CSSX (A) (7 $\mathrm{mM}$ MaxCalix with $0.75 \mathrm{M}$ Cs-7SB), and modified NB-CSSX (B) (7 mM MaxCalix with $0.5 \mathrm{M}$ Cs-7SB) is shown as a function of time.

Table 9.5. $D_{\mathrm{Cs}}$ for stripping as a function of time ${ }^{a}$

\begin{tabular}{cccc}
\hline Time (seconds) & \multicolumn{4}{c}{$\boldsymbol{D}_{\text {Cs }}$} \\
\hline & $\begin{array}{c}\text { CSSX } \\
\text { (7 mM BOBCalix) }\end{array}$ & $\begin{array}{c}\text { NG-CSSX } \\
\text { (50 } \mathbf{~ M ~ M a x C a l i x ) ~}\end{array}$ & $\begin{array}{c}\text { Modified NG-CSSX (B) } \\
\text { (7 mM MaxCalix) }\end{array}$ \\
\hline 5 & 0.644 & 0.087 & 0.078 \\
10 & 0.480 & 0.026 & 0.019 \\
15 & 0.31 & 0.00898 & 0.0048 \\
20 & 0.245 & 0.00428 & 0.0022 \\
30 & 0.179 & 0.00317 & 0.0007 \\
45 & 0.155 & 0.00233 & 0.0002 \\
60 & 0.123 & 0.00203 & 0.0003 \\
\hline
\end{tabular}

${ }^{\mathrm{a}}$ The solvent compositions are given in Table 9.3. Solvents were prepared by an extraction (O:A = 1:4) from SRS-15 simulant followed by two scrubs $(\mathrm{O}: \mathrm{A}=3.75: 1)$ at $25^{\circ} \mathrm{C}$ as described in the Experimental Section. The scrub and strip solutions for BOBCalixC6 were $0.050 \mathrm{M}$ and $0.001 \mathrm{M} \mathrm{HNO}_{3}$, respectively. The scrub and strip solutions for MaxCalix were $0.025 \mathrm{M}$ $\mathrm{NaOH}$ and $0.010 \mathrm{M} \mathrm{H}_{3} \mathrm{BO}_{3}$, respectively. The O:A ratios for all stripping contacts were 3.75:1, and all stripping contacts were run at $22-25^{\circ} \mathrm{C}$. 


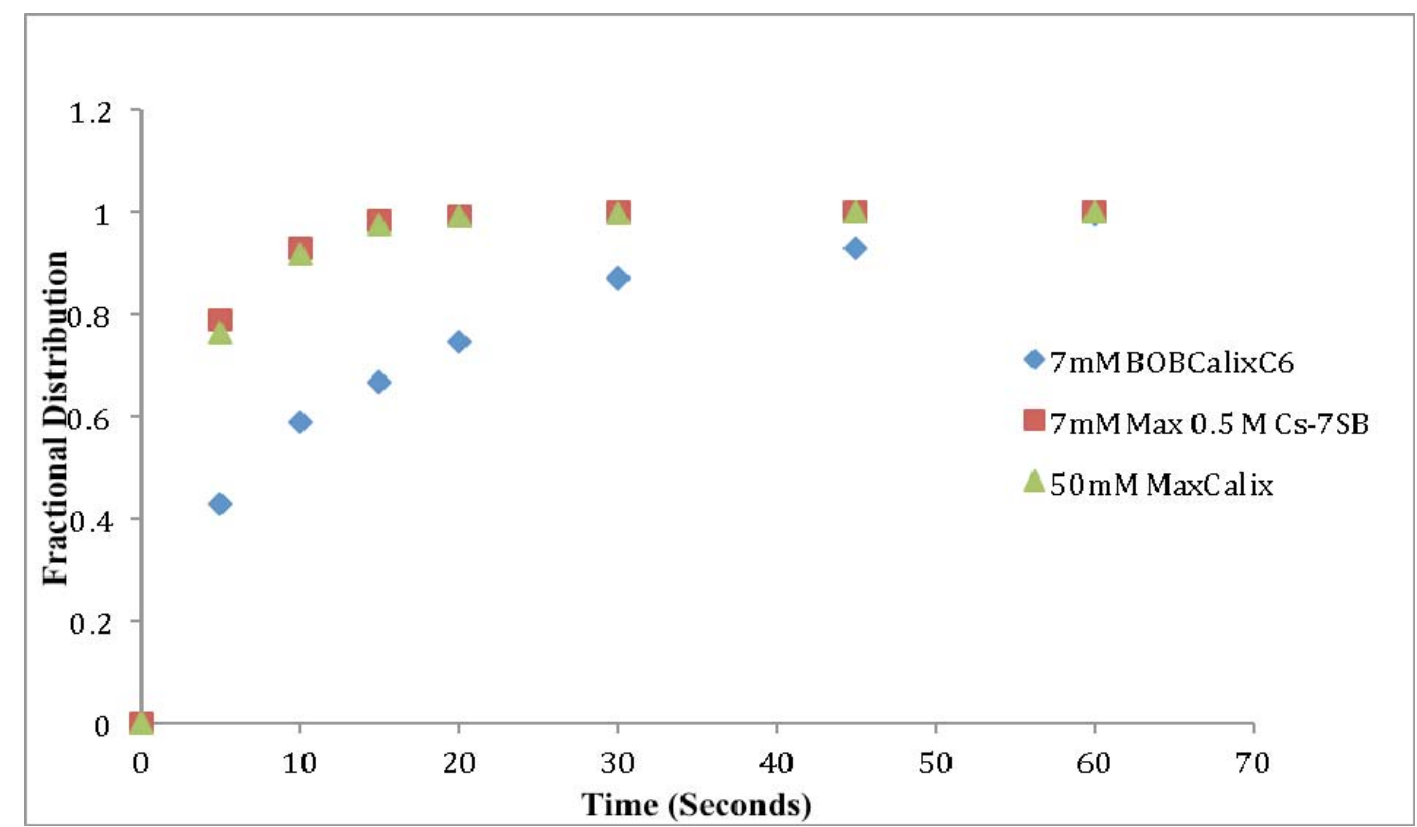

Fig. 9.2. Plot of fractional distribution versus time for stripping stage. Fractional distribution (Murphree efficiency) of NG-CSSX (50 mM MaxCalix), BOBCalixC6 CSSX (7 mM BOBCalix), and modified NG-CSSX (B) (7 mM MaxCalix with 0.5 $\mathrm{M}$ Cs-7SB) solvents is plotted as a function of time.

The slow stripping rate obtained here for the BOBCalixC6 CSSX solvent contrasts with an apparently faster result in early BOBCalixC6 CSSX development ${ }^{26}$. Examination of the conditions of the earlier test, however, cast doubt on the usefulness of any comparison here. Significant differences in technique are evident, hand-vortexing here while orbital vortexing in smaller tubes previously. It is perhaps more significant that the stripping chemistries differ. At the stage of solvent development in the early report ${ }^{26}$, the aqueous strip solution was $0.0001 \mathrm{M} \mathrm{CsNO}_{3}$ in $0.0005 \mathrm{M} \mathrm{HNO}_{3}$. Moreover, the BOBCalixC6 concentration was $10 \mathrm{mM}$, the modifier was an early candidate called Cs-3 used at $0.2 \mathrm{M}$, and there was no TOA in the solvent.

\subsection{CONCLUSIONS AND RECOMMENDATIONS}

Based on simple hand-vortexing experiments at $22-25^{\circ} \mathrm{C}$, the kinetics of NG-CSSX have been shown to be greatly improved in comparison with the current BOBCalixC6 CSSX process, dramatically so for stripping. On extraction, the time to reach $90 \%$ mass transfer for NG-CSSX is only $7 \mathrm{~s}$, less than half the time for BOBCalixC6 CSSX. The improvement is clearly due to the increased concentration of calixarene in the solvent, the modifier concentration having no detectable effect. On stripping, the time to reach $90 \%$ mass transfer for NG-CSSX is only $9 \mathrm{~s}$, less than a third of the time needed for BOBCalixC6 CSSX. The improvement is due to the different stripping chemistry of NG-CSSX, employing boric acid following a scrub with $0.025 \mathrm{M} \mathrm{NaOH}$. 
While the kinetics are interpreted here only at the phenomenological level, it is clear that the new chemistry of NG-CSSX markedly enhances mass-transfer rate in both extraction and stripping, portending good process stage efficiencies in centrifugal contactors. The faster kinetics are needed so that the operating NG-CSSX process using centrifugal contactors can take advantage of the much higher extraction $D_{\mathrm{Cs}}$ values and much lower stripping $D_{\mathrm{Cs}}$ values provided by the NG-CSSX chemistry to attain the high DFs required at the SRS. It is concluded that there should be no issues regarding chemical kinetics, and no further need for experimental work in the area of kinetics is identified. 



\section{NG-CSSX MASS TRANSFER PERFORMANCE IN A PARTIALLY PUMPING 5-CM CENTRIFUGAL CONTACTOR}

\subsection{INTRODUCTION}

This chapter describes testing designed to quantify the mass transfer performance of the NG-CSSX solvent in a 2-in (5-cm-diameter) centrifugal contactor relative to that provided by the predecessor BOBCalixC6 CSSX solvent. In this context, mass transfer testing has been performed using the same procedure, same equipment, and identical test conditions to the previous test $\mathrm{t}^{27}$. The test was designed to compare mass transfer performance obtained using BOBCalixC6 CSSX and NG-CSSX process chemistries, since uncertainty exists as to the ability to correlate contactor size with performance. The previous test indicated acceptable mass transfer performance that was later confirmed in results from operation of the larger-scale MCU. Lacking the ability to scale performance of 2-in commercial contactors to 5- and 10-in units used in the MCU, comparison of BOBCalixC6 CSSX and NG-CSSX results using identical 2-in test equipment coupled with demonstrated acceptable BOBCalixC6 CSSX performance in the larger MCU equipment is considered the most conclusive means of predicting acceptable NG-CSSX performance in MCU.

The work was driven by development and deployment of the improved solvent system for treatment of tank supernatant and dissolved salt wastes at the USDOE SRS as a replacement for the current chemistry. The BOBCalixC6 CSSX chemistry utilizes a solvent matrix comprising calix[4]arene-bis(tertoctylbenzocrown-6), aka BOBCalixC6, 1-(2,2,3,3-tetrafluoropropoxy),-3-[4-(sec-butyl)phenoxy]-2propanol (aka Cs-7SB), and tri-n-octylamine (TOA) in Isopar ${ }^{\circledR} \mathrm{L}$ (added as a diluent). Concentrations of the active components in the solvent matrix are $0.007 \mathrm{M}, 0.75 \mathrm{M}$, and $0.003 \mathrm{M}$, respectively. Cs-7SB acts to increase BOBCalixC solubility, preventing third-phase formation and facilitating increased $D_{C s}$; TOA suppresses impurity and ion-pair dissociation effects that would adversely impact stripping of cesium from the solvent. The modified chemistry (NG-CSSX) utilizes a reformulated solvent in which a monocrown calixarene, MaxCalix, replaces BOBCalixC6. The increased solubility of the former facilitates use of a higher concentration of the extractant in the solvent matrix, providing significantly higher $D_{\mathrm{Cs}}$ on extraction. In addition to the change in extractant, the NG-CSSX solvent utilizes a lower Cs-7SB concentration (intended to improve phase separation) and uses guanidine (in place of TOA) to suppress $D_{\mathrm{Cs}}$ under stripping conditions. In addition, dilute nitric acid used for cesium stripping in the BOBCalixC6 CSSX flowsheet is replaced with boric acid in NG-CSSX, primarily to minimize transfer of nitrate ions to downstream vitrification processing for waste immobilization. The change in strip solution 
necessitated a change in scrubbing; a caustic scrub $(\mathrm{NaOH})$ is used to achieve hydroxide displacement of extracted nitrate prior to stripping, thereby rejecting nitrate to the raffinate from extraction.

A limited set of hydraulic tests were conducted for the specific purpose of verifying adequate phase separation and mixing performance of the equipment used prior to the mass transfer test. In addition, extensive testing has been performed in an attempt to isolate the source(s) of cross-phase contamination that have been encountered during testing and operation of the centrifugal contactors used in the MCU.

The centrifugal contactor used in the study was a commercially available unit purchased from Costner Industries Nevada Company (CINC) in 2000, and was the standard CINC Model V-2 design at that time. Small modifications to the standard design have occurred since then, but should not affect mass transfer or hydraulic performance relative to the previous design. In any case, the contactor used in the reported test was the same unit used in BOBCalixC6 CSSX mass transfer studies between 2000 and 2002, thereby allowing direct comparison of mass transfer performance using BOBCalixC6 CSSX and NG-CSSX chemistries. Poor stage efficiencies from poor mixing during initial BOBCalixC6 CSSX testing were mitigated by modifying the contactor by increasing the size of the opening in the bottom of the rotor to make the contactor partially pumping. This modification increases liquid holdup in the mixing zone that, in turn, increases both the residence time and mixing intensity ${ }^{27}$. A similar modification was applied during one phase of NG-CSSX mass transfer testing. Straight bottom vanes were also used in both previous and current tests; these vanes produce more turbulent mixing than the curved vanes provided by the vendor as standard equipment.

\subsection{PURPOSE AND OBJECTIVES}

The changes that have been made to the process chemistry have the intended effect of altering mass transfer behavior in a multi-stage process as a result of changes in cesium partitioning between solvent and aqueous phases present in the ESS cascade. Additional changes affecting mass transfer stage efficiency may result from the change in solvent composition. Changes in droplet formation and coalescence behavior may alter specific interfacial area such that diffusion mass transfer mechanisms are impacted. Use of a different chemical compound as the extractant may impact the kinetics of formation of the calix-cesium complex. Quantification of the impacts of the changes in solvent composition and process chemistry (e.g. the change in strip solution from dilute $\mathrm{HNO}_{3}$ to $\mathrm{H}_{3} \mathrm{BO}_{3}$ ) is a prerequisite to design of multi-stage extraction cascade. In addition, the results of these tests may indicate needed refinement of the solvent formulation. 
Characterization of NG-CSSX chemistry and engineering performance required for design of a multistage flowsheet is being achieved through two facets of testing. One is focused strictly on fundamental chemical behavior: cesium partitioning as a function of solvent composition (including choice of extractant) and determination of extractant solubility. This work ${ }^{3}$ has proceeded to the point of selecting the new extractant and determining a formulation for near-term testing.

The experimental program performed and reported herein comprised determination of solvent physical properties, phase separation behavior, and hydraulic performance evaluations and mass transfer stage efficiency determinations in a pilot-scale centrifugal contactor. Property determinations were made to support selection of an extractant for the NG-CSSX application and to support equipment modeling and design activities. Phase separation behavior evaluations support solvent formula selection and contactor modeling. These tests are also prerequisite to mass transfer testing as they include determination of throughput for use in that effort. Mass transfer testing performed in a single contactor serves the purposes of verifying the efficacy of the process chemistry and quantifying stage efficiency in an apparatus representative of those used in full-scale processing. This last piece of data is essential for design of multi-stage processes for small-scale, real waste testing, and modeling and operation of production-scale systems.

A final component of the test program was an evaluation of factors potentially resulting in significant levels of effluent stream contamination with quantities of the opposing phase. The overall objectives of this element of testing were to isolate the source(s) of cross-phase contamination, to evaluate the impacts of operating conditions on the carryover problem, and to examine the effectiveness of contactor configuration modifications in mitigating the carryover problem.

Historically, centrifugal contactor-based solvent extraction processes have not demonstrated significant levels of contamination of effluents with the opposing phase except in instances in which the contactor weir configuration is not appropriate for the conditions in effect (flow rates, flow ratios, and phase separation characteristics), or when the combination of separator residence time and separation forces are inadequate to separate the dispersion created during mixing, due to the physical properties of the chemical system.

Significant limitations in contactor operation were first observed in centrifugal contactor hydraulic testing of the original BOBCalixC6 CSSX chemistry using 2-in diameter (Model V-2) centrifugal contactors procured from CINC. Carryover of solvent into the aqueous effluent stream was observed under 
conditions that were expected to generate contamination-free effluent, based on experience with similar chemistry (PUREX) in centrifugal contactors designed by ORNL, ANL and Savannah River Technology Center (SRTC, now the SRNL). Carryover of organic (less-dense) solution in the aqueous (more-dense) effluent was found to increase with increasing rotor speed, when the increase in separating power was expected to mitigate contamination. At the time it was suspected that the carryover was the result of back flow of organic phase foam, based on the observation of foam accumulation in the organic discharge nozzle.

Subsequent testing of CINC Model V-5 (5-in diameter) and Model V-10 (10-in diameter) rotors in support of MCU deployment indicated similar issues of cross-phase contamination, primarily the carryover of solvent in the aqueous effluent, that is exacerbated at both increased flow rate (expected) and increased rotor speed (unexpected). This problem has been mitigated during MCU operation by reducing system capacity to values well below the design capacity of the extraction equipment and by installing coalescers to remove entrained solvent from aqueous effluent streams. The ability to mitigate the problem in the MCU installation and/or future deployments (e.g., the SWPF) should facilitate increased throughput for any given size of contactor equipment, reduce the frequency of coalescer cleaning or replacement, and could potentially eliminate the need for coalescers altogether.

\subsection{PHYSICAL PROPERTY DETERMINATIONS}

Physical properties determined to support solvent formulation selection were density, viscosity, surface tension, and interfacial tension. Dispersion numbers, a dimensionless quantity that describes the tendency of a dispersion of two immiscible liquids to separate into its component phases, were also determined (Eq. 10.1). This quantity is determined by agitating the bulk solutions so as to generate a dispersion and measuring the time $t_{b}$ required for the thickness of the dispersion band to be reduced by a distance $z$ at an acceleration of $a$. In the work reported, predetermined volumes of aqueous and organic solutions were placed into a $100 \mathrm{~mL}$ graduated cylinder, a ground glass stopper was placed into the cylinder, and the solutions were manually shaken to create the dispersion. The vessel was vigorously shaken vertically for $20 \mathrm{~s}$, was allowed to stabilize for $10 \mathrm{~s}$, and was agitated for another $20 \mathrm{~s}$ interval, after which timing was initiated. In all cases, the method of agitation resulted in the entire depth of liquid becoming dispersed, and separation was timed until the interface between the two liquids returned to its original position and no individually distinguishable entrained droplets were visible in either phase. (However, timing was not continued until complete transparency of both bulk phases was restored.) Using the procedure described, $z$ becomes the total height of the liquid column in the graduated cylinder and $a$ is the gravitational constant. Where $\mathrm{N}_{\mathrm{Di}}$ values were obtained under extraction, scrubbing, stripping, and washing conditions, the 
determinations were made sequentially and the solvent was retained and reused in sequence in order to simulate solvent use in a full mass transfer cascade.

$$
N_{D t}=\frac{1}{\hbar_{b}} \sqrt{\frac{z}{\sigma}}
$$

As indicated by the expression, the dispersion number is inversely proportional to the time required for a band of dispersed liquids to separate into its component solutions, hence higher values of $\mathrm{N}_{\mathrm{Di}}$ indicate greater ease of separation.

The dispersion numbers obtained for a range of solvent formulations initially under consideration are presented in Table 10.1. Results in the table are for triplicate tests using three different extractants (BOBCalixC6, BEHBCalix, and mono-crown calixarene), both individually and in some combinations. In all cases, the background solvent matrix included $0.75 \mathrm{M}$ Cs-7SB and $0.003 \mathrm{M}$ TOA in Isopar L. The scrub and wash solutions used in the determinations were $0.1 \mathrm{M} \mathrm{NaOH}$. Strip solutions were boric acid at various concentrations; the values associated with the term 'Strip' in the tables are $\mathrm{H}_{3} \mathrm{BO}_{3}$ concentrations expressed as molarities. All dispersion numbers reported were obtained at $25{ }^{\circ} \mathrm{C}$. While there are no conditions under which phase separation could not be achieved in centrifugal contactors, low dispersion numbers when the solvent is dispersed in very dilute $(1 \mathrm{mM}) \mathrm{H}_{3} \mathrm{BO}_{3}$ stripping solution indicate a possible need to significantly limit throughput in stripping to obtain the residence time required for adequate separation to be achieved.

Subsequent to the decision to focus on mono-crown solvent formulations, additional dispersion number determinations were performed to examine the effects of modifier (Cs-7SB) concentration, the use of guanidine in place of TOA as a suppressor, and phase ratio on phase separation performance. Results of these determinations are presented in Tables $10.2-10.4$.

Data in Table 10.2 indicates consistent improvements in phase separation performance with decreased modifier concentration under conditions of extraction, scrubbing, stripping and solvent washing. Data presented in Table 10.3 were obtained to evaluate the effect of modified phase ratios on separation behavior. Phase ratio modifications from the current baseline are intended to improve phase separation performance by reducing throughput and by increasing residence time in the separating zone of the contactors. The $\mathrm{N}_{\mathrm{Di}}$ results obtained are in the same general range as those obtained for all candidate solvents when the baseline phase ratios were applied (Table 10.1), but are lower than those obtained for 
$20 \mathrm{mM}$ MaxCalix at various modifier concentrations (Table 10.2). Based on the results, it is unlikely that that modified phase ratios will have significant impacts on phase separation performance.

Table 10.1. Dispersion numbers for candidate solvents*

\begin{tabular}{|c|c|c|c|c|c|c|}
\hline \multicolumn{2}{|l|}{ Extractant } & \multicolumn{3}{|c|}{ - } & \multirow{2}{*}{$\frac{\text { Avg. } \mathrm{t}_{\mathrm{b}}, \mathrm{s}}{146.7}$} & \multirow{2}{*}{$\frac{\mathrm{N}_{\mathrm{Di}}}{8.23 \times 10^{-4}}$} \\
\hline $20 \mathrm{mM}$ BEHB & Extraction & 155 & 142 & 143 & & \\
\hline & Scrub & 255 & 250 & 242 & 249.0 & $4.45 \times 10^{-4}$ \\
\hline & Strip 0.001 & 520 & 510 & 510 & 513.3 & $2.16 \times 10^{-4}$ \\
\hline & Strip 0.01 & 320 & 340 & 335 & 331.7 & $3.34 \times 10^{-4}$ \\
\hline & Strip 0.1 & 226 & 210 & 253 & 229.7 & $4.83 \times 10^{-4}$ \\
\hline & Wash & 290 & 288 & 250 & 276.0 & $3.91 \times 10^{-4}$ \\
\hline \multirow[t]{7}{*}{$20 \mathrm{mM}$ Mono } & Extraction & 124 & 125 & 140 & 129.7 & $9.31 \times 10^{-4}$ \\
\hline & Scrub & 140 & 195 & 300 & 211.7 & $5.24 \times 10^{-4}$ \\
\hline & Strip 0.001 & 460 & 462 & 510 & 477.3 & $2.32 \times 10^{-4}$ \\
\hline & Strip 0.01 & 445 & 425 & 385 & 418.3 & $2.65 \times 10^{-4}$ \\
\hline & Strip 0.1 & 195 & 250 & 220 & 221.7 & $5.00 \times 10^{-4}$ \\
\hline & Strip 0.5 & 90 & 110 & 95 & 98.3 & $1.13 \times 10^{-3}$ \\
\hline & Wash & 305 & 295 & 305 & 301.7 & $3.58 \times 10^{-4}$ \\
\hline \multirow[t]{6}{*}{$7 \mathrm{mM} \mathrm{BoB}$} & Extraction & 150 & 154 & 145 & 149.7 & $8.06 \times 10^{-4}$ \\
\hline & Scrub & 310 & 280 & 288 & 292.7 & $3.79 \times 10^{-4}$ \\
\hline & Strip 0.001 & 300 & 290 & 285 & 291.7 & $3.80 \times 10^{-4}$ \\
\hline & Strip 0.01 & 125 & 133 & 142 & 133.3 & $8.32 \times 10^{-4}$ \\
\hline & Strip 0.1 & 195 & 165 & 180 & 180.0 & $6.16 \times 10^{-4}$ \\
\hline & Wash & 148 & 188 & 190 & 175.3 & $6.16 \times 10^{-4}$ \\
\hline \multirow[t]{6}{*}{$7 \mathrm{mM}$ BEHB } & Extraction & 134 & 135 & 135 & 134.7 & $8.96 \times 10^{-4}$ \\
\hline & Scrub & 162 & 186 & 182 & 176.7 & $6.28 \times 10^{-4}$ \\
\hline & Strip 0.001 & 190 & 178 & 173 & 180.3 & $6.15 \times 10^{-4}$ \\
\hline & Strip 0.01 & 170 & 162 & 175 & 169.0 & $6.56 \times 10^{-4}$ \\
\hline & Strip 0.1 & 170 & 145 & 160 & 158.3 & $7.00 \times 10^{-4}$ \\
\hline & Wash & 120 & 128 & 130 & 126.0 & $8.57 \times 10^{-4}$ \\
\hline \multirow[t]{6}{*}{$7 \mathrm{mM}$ Mono } & Extraction & 137 & 133 & 136 & 135.3 & $8.92 \times 10^{-4}$ \\
\hline & Scrub & 205 & 200 & 312 & 239.0 & $4.64 \times 10^{-4}$ \\
\hline & Strip 0.001 & 410 & 396 & 390 & 398.7 & $2.78 \times 10^{-4}$ \\
\hline & Strip 0.01 & 135 & 142 & 153 & 143.3 & $7.74 \times 10^{-4}$ \\
\hline & Strip 0.1 & 230 & 220 & 240 & 230.0 & $4.82 \times 10^{-4}$ \\
\hline & Wash & 185 & 210 & 190 & 195.0 & $5.54 \times 10^{-4}$ \\
\hline $7 \mathrm{mM}$ BoB and & Extraction & 118 & 123 & 118 & 119.7 & $1.01 \times 10^{-3}$ \\
\hline \multirow[t]{5}{*}{$13 \mathrm{mM}$ BEHB } & Scrub & 248 & 276 & 285 & 269.7 & $4.11 \times 10^{-4}$ \\
\hline & Strip 0.001 & 360 & 360 & 445 & 388.3 & $2.86 \times 10^{-4}$ \\
\hline & Strip 0.01 & 165 & 188 & 172 & 175.0 & $6.34 \times 10^{-4}$ \\
\hline & Strip 0.1 & 190 & 185 & 210 & 195.0 & $5.69 \times 10^{-4}$ \\
\hline & Wash & 160 & 170 & 75 & 135.0 & $8.00 \times 10^{-4}$ \\
\hline \multirow{6}{*}{$13 \mathrm{mM}$ Mono } & Extraction & 137 & 128 & 130 & 131.7 & $9.17 \times 10^{-4}$ \\
\hline & Scrub & 200 & 216 & 114 & 176.7 & $6.28 \times 10^{-4}$ \\
\hline & Strip 0.001 & 480 & 515 & 530 & 508.3 & $2.18 \times 10^{-4}$ \\
\hline & Strip 0.01 & 320 & 245 & 290 & 285.0 & $3.89 \times 10^{-4}$ \\
\hline & Strip 0.1 & 195 & 225 & 226 & 215.3 & $5.15 \times 10^{-4}$ \\
\hline & Wash & 225 & 225 & 225 & 225.0 & $4.80 \times 10^{-4}$ \\
\hline
\end{tabular}

"Organic-to-aqueous phase ratios applied were 1:3 for extraction and 5:1 for scrubbing, stripping and washing. 
Table 10.2. Effect of modifier concentration on dispersion number*

(20 mM mono-crown calix, $3 \mathrm{mM}$ guanidine suppressor)

\begin{tabular}{clccccc}
\hline $\begin{array}{c}\text { Modifier } \\
\text { concentration }\end{array}$ & & & & & & \\
& & $----------\mathbf{t}_{\mathbf{b}}, \mathbf{s}$-------- & Avg. $\mathbf{t}_{\mathbf{b}}, \mathbf{s}$ & $\mathbf{N}_{\mathbf{D i}}$ \\
\hline 0.75 M Cs-7SB & Extraction & 115 & 118 & 115 & 116.0 & $1.04 \times 10^{-3}$ \\
& Scrub & 170 & 165 & 175 & 170.0 & $6.52 \times 10^{-4}$ \\
& Strip 0.1 & 146 & 118 & 155 & 139.7 & $7.94 \times 10^{-4}$ \\
& Wash & 260 & 260 & 260 & 260.0 & $4.15 \times 10^{-4}$ \\
\hline 0.5 M Cs-7SB & Extraction & 104 & 100 & 102 & 102.0 & $1.18 \times 10^{-3}$ \\
& Scrub & 105 & 105 & 120 & 110.0 & $1.01 \times 10^{-3}$ \\
& Strip 0.1 & 125 & 130 & 105 & 120.0 & $9.24 \times 10^{-4}$ \\
& Wash & 110 & 100 & 100 & 103.3 & $1.04 \times 10^{-3}$ \\
\hline 0.25 M Cs-7SB & Extraction & 90 & 82 & 87 & 86.3 & $1.40 \times 10^{-3}$ \\
& Scrub & 80 & 73 & 77 & 76.7 & $1.45 \times 10^{-3}$ \\
& Strip 0.1 & 105 & 90 & 85 & 93.3 & $1.19 \times 10^{-3}$ \\
& Wash & 60 & 66 & 65 & 63.7 & $1.70 \times 10^{-3}$ \\
\hline
\end{tabular}

"Organic-to-aqueous phase ratios applied were 1:3 for extraction and 5:1 for scrubbing, stripping and washing.

Table 10.3. Dispersion numbers at modified phase ratios

(50 mM mono-crown calix, $3 \mathrm{mM}$ guanidine suppressor)

\begin{tabular}{|c|c|c|c|c|c|c|}
\hline \multicolumn{2}{|c|}{ Phase ratio } & \multicolumn{3}{|c|}{---------t } & \multirow{2}{*}{$\frac{\text { Avg. } \mathbf{t}_{\mathbf{b}}, \mathbf{s}}{121.3}$} & $\mathbf{N}_{\text {Di }}$ \\
\hline $1: 4$ & Extraction & 118 & 122 & 124 & & $9.95 \times 10^{-4}$ \\
\hline $3.75: 1$ & Scrub $25 \mathrm{mM} \mathrm{NaOH}$ & 145 & 152 & 167 & 154.7 & $6.78 \times 10^{-4}$ \\
\hline $3.75: 1$ & Strip $10 \mathrm{mM} \mathrm{H}_{3} \mathrm{BO}_{3}$ & 145 & 130 & 120 & 131.7 & $7.36 \times 10^{-4}$ \\
\hline $5: 1$ & Wash $10 \mathrm{mM} \mathrm{NaOH}$ & 147 & 134 & 127 & 136.0 & $6.61 \times 10^{-4}$ \\
\hline $3.75: 1$ & Wash $10 \mathrm{mM} \mathrm{NaOH}$ & 176 & 157 & 150 & 161.0 & $5.47 \times 10^{-4}$ \\
\hline
\end{tabular}

Evaluations of phase separation behavior as a function of scrub solution concentration and solvent-toscrub phase ratio were performed to determine if latitude exists to vary these parameters. The results of this test are presented in Table 10.4. Taken as a whole, dispersion numbers obtained show little difference from those determined under other conditions considered previously. Individually, there is slight improvement in phase separation associated with increased $\mathrm{NaOH}$ concentration, as was anticipated. However, all values obtained are indicative of solution pairs that can be effectively separated in centrifugal contactors.

Table 10.4. Effects of scrub solution concentration and phase ratio on dispersion number

(50 mM mono-crown calix, $3 \mathrm{mM}$ guanidine suppressor)

\begin{tabular}{ccccccc}
\hline Solution & Phase ratio & $---------\mathbf{t}_{\mathbf{b}}, \mathbf{s}$-------- & Avg. $\mathbf{t}_{\mathbf{b}}, \mathbf{s}$ & $\mathbf{N}_{\mathbf{D i}}$ \\
\hline $25 \mathrm{mM} \mathrm{NaOH} \mathrm{Scrub}$ & $5: 1$ & 165 & 172 & 190 & 175.7 & $6.31 \times 10^{-4}$ \\
$25 \mathrm{mM} \mathrm{NaOH} \mathrm{Scrub}$ & $2.5: 1$ & 150 & 183 & 190 & 174.3 & $6.36 \times 10^{-4}$ \\
$50 \mathrm{mM} \mathrm{NaOH} \mathrm{Scrub}$ & $5: 1$ & 165 & 160 & 162 & 162.3 & $6.83 \times 10^{-4}$ \\
$50 \mathrm{mM} \mathrm{NaOH}$ Scrub & $2.5: 1$ & 135 & 127 & 143 & 135.0 & $8.21 \times 10^{-4}$ \\
\hline
\end{tabular}


Additional physical property data was obtained to support modeling activities and to provide additional information supporting selection of a solvent formulation. Those data are presented in Tables 10.5 through 10.8. Of perhaps most interest are the interfacial tension values in Table 10.8. These data are consistent with dispersion number trends. Higher interfacial tension values under the extraction condition (as opposed to scrubbing, stripping and washing), which are indicative of stable interfaces that are more difficult to break apart, are also reflected in higher dispersion numbers that indicate less resistance to dispersion separation.

Table 10.5. Solution densities

\begin{tabular}{llcc}
\hline \multicolumn{1}{c}{ Solution } & $\begin{array}{c}\text { Suppressor } \\
\text { (if solvent) }\end{array}$ & Modifier [M] & Density [g/cc] \\
\hline $20 \mathrm{mM} \mathrm{Max}$ & $3 \mathrm{mM}$ TOA & 0.5 & 0.821 \\
$30 \mathrm{mM} \mathrm{Max}$ & $3 \mathrm{mM}$ DCiTG & 0.5 & 0.824 \\
$50 \mathrm{mM} \mathrm{Max}$ & $3 \mathrm{mM}$ DCiTG & 0.5 & 0.826 \\
$7 \mathrm{mM} \mathrm{BoB}$ & $3 \mathrm{mM} \mathrm{DCiTG}$ & 0.75 & 0.846 \\
$7 \mathrm{mM} \mathrm{BoB}$ & $3 \mathrm{mM} \mathrm{TOA}$ & 0.75 & 0.850 \\
$\mathrm{SRS}-15 \mathrm{Simulant}$ & & & 1.242 \\
$25 \mathrm{mM} \mathrm{NaOH}$ & & & 1.005 \\
$10 \mathrm{mM} \mathrm{NaOH}$ & & & 1.001 \\
$10 \mathrm{mM} \mathrm{H} \mathrm{BO}_{3}$ & & & 1.002 \\
\hline
\end{tabular}

Table 10.6. Solution viscosities

\begin{tabular}{llccccc}
\hline \multicolumn{1}{c}{ Solution } & $\begin{array}{c}\text { Suppressor } \\
\text { (if solvent) }\end{array}$ & $\begin{array}{c}\text { Modifier } \\
{[\boldsymbol{M}]}\end{array}$ & $\begin{array}{c}\text { Viscosity } \\
{[\mathbf{c P}]}\end{array}$ & $\begin{array}{c}\text { Viscometer } \\
\text { spindle rpm }\end{array}$ & $\begin{array}{c}\text { Viscosity } \\
{[\mathbf{c P}]}\end{array}$ & $\begin{array}{c}\text { Viscometer } \\
\text { spindle rpm }\end{array}$ \\
\hline $20 \mathrm{mM} \mathrm{Max}$ & $3 \mathrm{mM} \mathrm{TOA}$ & 0.5 & 2.76 & 50 & 2.8 & 100 \\
$30 \mathrm{mM} \mathrm{Max}$ & $3 \mathrm{mM} \mathrm{DCiTG}$ & 0.5 & 2.44 & 50 & 2.45 & 100 \\
$50 \mathrm{mM} \mathrm{Max}$ & $3 \mathrm{mM} \mathrm{DCiTG}$ & 0.5 & 2.75 & 50 & 2.77 & 100 \\
$7 \mathrm{mM} \mathrm{BoB}$ & $3 \mathrm{mM} \mathrm{DCiTG}$ & 0.75 & 3.55 & 50 & 3.56 & 100 \\
$7 \mathrm{mM} \mathrm{BoB}$ & $3 \mathrm{mM} \mathrm{TOA}$ & 0.75 & 3.5 & 50 & 3.44 & 100 \\
$\mathrm{SRS}-15 \mathrm{Simulant}$ & & & 2.6 & 60 & 2.62 & 100 \\
$25 \mathrm{mM} \mathrm{NaOH}$ & & & 1.01 & 60 & 1.34 & 100 \\
$10 \mathrm{mM} \mathrm{NaOH}$ & & & 0.97 & 60 & 1.31 & 100 \\
$10 \mathrm{mM} \mathrm{H} \mathrm{BO}_{3}$ & & & 1 & 60 & 1.31 & 100 \\
\hline
\end{tabular}

Table 10.7. Solution surface tensions

\begin{tabular}{llccccc}
\hline \multicolumn{1}{c}{ Solution } & $\begin{array}{c}\text { Suppressor } \\
\text { (if solvent) }\end{array}$ & Modifier $[\boldsymbol{M}$ ] & -----Surface Tension----- & \\
[dynes/cm] & & Average: \\
\hline $20 \mathrm{mM} \mathrm{Max}$ & $3 \mathrm{mM}$ TOA & 0.5 & 26 & 26.5 & 26 & 26.16 \\
$30 \mathrm{mM} \mathrm{Max}$ & $3 \mathrm{mM} \mathrm{DCiTG}$ & 0.5 & 25 & 25 & 25.5 & 25.16 \\
$50 \mathrm{mM} \mathrm{Max}$ & $3 \mathrm{mM} \mathrm{DCiTG}$ & 0.5 & 24 & 25 & 25 & 24.66 \\
$7 \mathrm{mM} \mathrm{BoB}$ & $3 \mathrm{mM} \mathrm{DCiTG}$ & 0.75 & 26 & 26 & 26 & 26 \\
$7 \mathrm{mM} \mathrm{BoB}$ & $3 \mathrm{mM} \mathrm{TOA}$ & 0.75 & 26 & 26 & 25.5 & 25.83 \\
$\mathrm{SRS}-15 \mathrm{Simulant}$ & & & 40.5 & 42 & 42 & 41.5 \\
$25 \mathrm{mM} \mathrm{NaOH}$ & & & 50.5 & 51 & 53 & 51.5 \\
$10 \mathrm{mM} \mathrm{NaOH}$ & & & 49 & 52 & 50 & 50.33 \\
$10 \mathrm{mM} \mathrm{H}_{3} \mathrm{BO}_{3}$ & & & 50 & 50 & 52 & 50.66 \\
\hline
\end{tabular}


Table 10.8. System interfacial tensions

\begin{tabular}{|c|c|c|c|c|c|c|c|}
\hline \multirow{2}{*}{$\frac{\text { Extractant }}{20 \mathrm{mM} \mathrm{Max}}$} & \multirow{2}{*}{$\frac{\text { Suppressor }}{3 \mathrm{mM} \text { TOA }}$} & \multirow{2}{*}{$\frac{\text { Modifier }[M]}{0.5}$} & Aqueous sol'n & \multicolumn{3}{|c|}{$\begin{array}{c}\text {----Interfacial Tension---- } \\
\text { [dynes/cm] }\end{array}$} & \multirow{2}{*}{$\begin{array}{c}\begin{array}{c}\text { Average } \\
{[\text { dynes/cm }}\end{array} \\
20\end{array}$} \\
\hline & & & SRS-15 Simulant & 20 & 22 & 18 & \\
\hline & & & 25 mM Scrub & 15 & 14.5 & 16 & 15.16 \\
\hline & & & 100 mM Strip & 14 & 16 & 17 & 15.66 \\
\hline & & & $10 \mathrm{mM}$ Wash & 15 & 16 & 16 & 15.66 \\
\hline \multirow[t]{5}{*}{$30 \mathrm{mM}$ Max } & $3 \mathrm{mM}$ & 0.5 & & & & & \\
\hline & DCiTG & & SRS-15 Simulant & 11 & 12 & 11 & 11.33 \\
\hline & & & 25 mM Scrub & 10 & 11 & 12 & 11 \\
\hline & & & 100 mM Strip & 14 & 14 & 13 & 13.66 \\
\hline & & & $10 \mathrm{mM}$ Wash & 12 & 13 & 12 & 12.33 \\
\hline \multirow[t]{5}{*}{$50 \mathrm{mM}$ Max } & $3 \mathrm{mM}$ & 0.5 & & & & & \\
\hline & DCiTG & & SRS-15 Simulant & 19 & 18 & 18 & 18.33 \\
\hline & & & 25 mM Scrub & 17 & 16 & 17 & 16.66 \\
\hline & & & 100 mM Strip & 12 & 14 & 15 & 13.66 \\
\hline & & & $10 \mathrm{mM}$ Wash & 13 & 13 & 15 & 13.66 \\
\hline \multirow[t]{5}{*}{$50 \mathrm{mM}$ Max } & $3 \mathrm{mM}$ & 0.5 & & & & & \\
\hline & DCiTG & & SRS-15 Simulant & 19 & 18 & 18 & 18.33 \\
\hline & & & 25 mM Scrub & 17 & 16 & 17 & 16.66 \\
\hline & & & 10 mM Strip & & & & NA \\
\hline & & & $10 \mathrm{mM}$ Wash & & & & NA \\
\hline \multirow[t]{5}{*}{$7 \mathrm{mM}$ BoB } & $3 \mathrm{mM}$ & 0.75 & & & & & \\
\hline & DCiTG & & SRS-15 Simulant & 20 & 18 & 16 & 18 \\
\hline & & & 25 mM Scrub & 15.5 & 16 & 16 & 15.83 \\
\hline & & & 100 mM Strip & 16 & 16 & 15 & 15.66 \\
\hline & & & $10 \mathrm{mM}$ Wash & 14 & 15 & 15.5 & 14.83 \\
\hline \multirow[t]{4}{*}{$7 \mathrm{mM}$ BoB } & $3 \mathrm{mM}$ TOA & 0.75 & SRS-15 Simulant & 19 & 16 & 17 & 17.33 \\
\hline & & & 25 mM Scrub & 14 & 13 & 14 & 13.66 \\
\hline & & & 100 mM Strip & 14.5 & 15 & 13.5 & 14.33 \\
\hline & & & $10 \mathrm{mM}$ Wash & 15 & 16 & 16 & 15.66 \\
\hline
\end{tabular}

\subsection{PRELIMINARY HYDRAULIC PERFORMANCE EVALUATION}

\subsubsection{Reagents Used}

The solvent formulation used in both mass transfer testing and in the associated, preliminary contactor hydraulic test is listed in Table $10.9^{3}$. SRS-15 tank waste simulant (Table 10.10) was used in extractioncondition testing. The scrub solution used in all hydraulic testing was $25 \mathrm{mM} \mathrm{NaOH}$. Strip-condition testing was performed using $10 \mathrm{mM} \mathrm{H}_{3} \mathrm{BO}_{3}$.

\subsubsection{Procedure}

A series of tests were performed to determine phase separation performance using a pilot-scale centrifugal contactor operating under extraction, scrubbing, stripping, and solvent washing conditions. The device used was a CINC Model V-2 centrifugal solvent extraction contactor (CINC) of the manufacturer's standard design with the exception of the vane configuration located beneath the contactor rotor. As received, CINC contactors are equipped with housing end plates that have eight, curved vanes machined 
into the upper surface so as to be located in close proximity to the bottom face of the rotor, once installed.

As is the case with all lower vanes used in centrifugal contactors, these are intended to prevent vortex formation on the rotor's bottom surface at the axis of rotation. Creation of a vortex in this location has been found to be very effective in preventing flow of solution into the rotor, resulting in flooding of the apparatus. The additional intent of curved vanes (which are curved inward, in the direction of rotor rotation) is to direct flow into the rotor, thereby reducing turbulence at the bottom of the rotor to enhance phase separation. Most contactor testing reported here was performed using a bottom cap having eight, straight, radial vanes, as this configuration provides better mixing than the curved vane approach, which results in increased solute transfer efficiency. For comparison purposes, some extraction-condition hydraulic testing was performed using curved vanes (as noted in the presentation of results).

Table 10.9. NG-CSSX formulation

\begin{tabular}{|c|c|c|c|c|}
\hline Component & Concentration & Code & Chemical Name & Structure \\
\hline Extractant & $0.050 \mathrm{M}$ & MaxCalix & $\begin{array}{l}\text { 1,3-alt-25,27-Bis(3,7- } \\
\text { dimethyloctyl-1-oxy) } \\
\text { calix[4]arene- } \\
\text { benzocrown- } 6\end{array}$ & \\
\hline Modifier & $0.50 M$ & Cs-7SB & $\begin{array}{l}\text { 1-(2,2,3,3- } \\
\text { Tetrafluoropropoxy)-3- } \\
\text { (4-sec-butylphenoxy)-2- } \\
\text { propanol }\end{array}$ & \\
\hline Suppressor & $0.003 M$ & LIX 79 & $\begin{array}{l}N, N \text {-Dicyclohexyl- } N " \text { - } \\
\text { isotridecylguanidine }\end{array}$ & \\
\hline Diluent & & Isopar L & $\mathrm{C}_{12}$-isoparaffinic hydrocarbon & \\
\hline
\end{tabular}

Table 10.10. Target concentration of SRS-15 simulant

\begin{tabular}{ll}
\hline \multicolumn{1}{c}{ Specie } & \multicolumn{1}{c}{$\begin{array}{c}\text { Concentration, } \\
\boldsymbol{M}\end{array}$} \\
\hline Principal constituents: & \\
$\mathrm{Al}$ as $\left(\mathrm{Al}(\mathrm{OH})_{4}{ }^{-}\right)$ & 0.280 \\
$\mathrm{Cl}^{-}$ & $2.4 \times 10^{-2}$ \\
$\mathrm{CO}_{3}{ }^{2-}$ (TIC) & 0.150 \\
$\mathrm{Cs}^{+}$(Total) & $1.4 \times 10^{-4}$ \\
$\mathrm{~K}^{+}$ & 0.015 \\
$\mathrm{Na}^{+}$ & 5.60 \\
$\mathrm{NO}_{2}{ }^{-}$ & 0.500 \\
$\mathrm{NO}_{3}{ }^{-}$ & 2.03 \\
$\mathrm{OH}^{-}$(Free) & 2.06 \\
$\mathrm{SO}_{4}{ }^{2-}$ & 0.140 \\
\hline $\mathrm{Minor}$ inorganic constituents: & \\
$\mathrm{Ag}(\mathrm{I})$ & $9.3 \times 10^{-8}$ \\
$\mathrm{Bi}(\mathrm{III})$ &
\end{tabular}


Table 10.10. Target concentration of SRS-15 simulant

\begin{tabular}{|c|c|}
\hline Specie & $\begin{array}{c}\text { Concentration, } \\
M\end{array}$ \\
\hline \multicolumn{2}{|l|}{$\mathrm{Ca}^{2+}$} \\
\hline $\mathrm{CrO}_{4}^{2-}$ & $1.4 \times 10^{-3}$ \\
\hline $\mathrm{Cu}(\mathrm{II})$ & $2.3 \times 10^{-5}$ \\
\hline $\mathrm{F}^{-}$ & $2.8 \times 10^{-2}$ \\
\hline $\mathrm{Fe}(\mathrm{III})$ & $2.6 \times 10^{-5}$ \\
\hline $\operatorname{Hg}(\mathrm{II})$ & $2.5 \times 10^{-7}$ \\
\hline \multicolumn{2}{|l|}{$\mathrm{La}(\mathrm{III})$} \\
\hline \multicolumn{2}{|l|}{$\mathrm{Mn}(\mathrm{VII})$} \\
\hline $\mathrm{MoO}_{4}^{2-}$ & $7.0 \times 10^{-5}$ \\
\hline \multicolumn{2}{|l|}{$\mathrm{Ni}(\mathrm{II})$} \\
\hline $\mathrm{NH}_{3}$ & $1.0 \times 10^{-3}$ \\
\hline $\mathrm{Pb}(\mathrm{II})$ & $1.0 \times 10^{-5}$ \\
\hline $\mathrm{Pd}(\mathrm{II})$ & $3.8 \times 10^{-6}$ \\
\hline $\mathrm{PO}_{4}^{3-}$ & $7.0 \times 10^{-3}$ \\
\hline $\mathrm{Rh}(\mathrm{III})$ & $2.0 \times 10^{-6}$ \\
\hline $\mathrm{Ru}(\mathrm{III})$ & $8.1 \times 10^{-6}$ \\
\hline $\mathrm{Si}(\mathrm{IV})$ & $3.0 \times 10^{-2}$ \\
\hline $\mathrm{Sn}(\mathrm{II})$ & $2.0 \times 10^{-5}$ \\
\hline \multicolumn{2}{|l|}{$\mathrm{Sr}^{2+}$} \\
\hline $\mathrm{Zn}(\mathrm{II})$ & $1.2 \times 10^{-4}$ \\
\hline $\mathrm{Zr}(\mathrm{IV})$ & \\
\hline \multicolumn{2}{|l|}{ Organic constituents: } \\
\hline$n$-Butanol & $2.7 \times 10^{-5}$ \\
\hline Tri-n-butylphosphate (TBP) & $1.9 \times 10^{-6}$ \\
\hline Di-n-butylphosphate (DBP) & $1.2 \times 10^{-4}$ \\
\hline Mono-n-butylphosphate (MBP) & $1.6 \times 10^{-4}$ \\
\hline Formate $\left(\mathrm{HCO}_{2}^{-}\right)$ & $3.3 \times 10^{-2}$ \\
\hline Oxalate $\left(\mathrm{C}_{2} \mathrm{O}_{4}{ }^{2-}\right)$ & $8.0 \times 10^{-3}$ \\
\hline Trimethylamine & $1.7 \times 10^{-4}$ \\
\hline
\end{tabular}

Testing was performed in a closed loop configuration. Under any particular set of conditions, the solvent phase was placed in one supply vessel, which also served as the solvent collection vessel. A similar arrangement was used for aqueous feed solution supply and collection. Solutions were transferred to the contactor using Cole-Parmer Micropumps (Model 75211-30, manufactured by the Barnant Co.). The pumps were variable speed gear-type, equipped with N23 gear heads, capable of delivering flows of 40$2400 \mathrm{~mL} / \mathrm{min}$ controllable in $10 \mathrm{~mL} / \mathrm{min}$ increments. Most testing was performed using a heavy-phase weir having a diameter of 1.00 in. Some additional testing was performed with weirs of different sizes when results indicated poor phase separation. Similarly, most testing was performed with the rotor operating at $3600 \mathrm{rpm}$. When indicated by test results, some variation of rotor speed was used.

In all cases, contactor operation at the desired speed was initiated, followed by introduction of the aqueous feed solution. The flow of the organic solution was started once discharge of aqueous flow from 
the contactor's upper collector ring was observed. Extraction-condition testing was performed at organicto-aqueous phase ratios of 1:2,1:3, and 1:4; variations from the 1:3 BOBCalixC6 CSSX baseline were considered to evaluate the ability to increase waste processing throughput by increasing the aqueous feed rate without increasing total (aqueous + organic) contactor throughput. Scrub-condition testing was performed at phase ratios of 5:1 (the current baseline) and 3.75:1. Strip-condition evaluations were performed at phase ratios of $3.75: 1,4: 1$, and 5:1 (the current baseline).

It is important to note that the standard practice at the start of testing was to maintain a specific flow condition for 2-3 minutes before collecting samples of the effluents for visual examination for entrainment. If no entrainment in the form of visibly discernable droplets of carryover were observed, or if no organic film or sheen was seen on the surface of an aqueous sample, flow rates were increased and additional samples were collected. If entrainment was apparent, flow rates were reduced until acceptable performance was reestablished. Near the end of testing, the interval of operation at a flow condition was extended, as described below.

\subsubsection{Hydraulic Testing Observations}

\subsubsection{Extraction}

Initial testing was performed using a contactor equipped with curved lower vanes. Testing was conducted at a phase ratio of $1: 2$, using a rotor fitted with a $1.0 \mathrm{in}$. heavy phase weir operating at $3600 \mathrm{rpm}$. No carryover was observed in either effluent solution at total flows up to $750 \mathrm{~mL} / \mathrm{min}$. The appearance of a slight sheen on the aqueous effluent was observed at a total flow rate of $1050 \mathrm{~mL} / \mathrm{min}$. Distinguishable droplets of organic were seen on the surface of an aqueous effluent sample at a total throughput of $2100 \mathrm{~mL} / \mathrm{min}$. At this point, flows were reduced and reset to provide a phase ratio of 1:3. No entrainment was observed at total flow rates up to $1200 \mathrm{~mL} / \mathrm{min}$. A sheen was observed on top of an aqueous phase sample collected at a total throughput of $1480 \mathrm{~mL} / \mathrm{min}(370 \mathrm{~mL} / \mathrm{min}$ organic: $1110 \mathrm{~mL} / \mathrm{min}$ aqueous $)$. The same observation was made at $1600 \mathrm{~mL} / \mathrm{min}$; distinct organic droplets were observed in the aqueous effluent at a total throughput of $1840 \mathrm{~mL} / \mathrm{min}$. At this point, flows were reduced and reset to obtain a throughput phase ratio of 1:4. Testing was initiated at $500 \mathrm{~mL} / \mathrm{min}$ total throughput. Flows were increased incrementally (in $100 \mathrm{~mL} / \mathrm{min}$ increments) and samples were collected for examination. Solvent droplets were observed in aqueous samples collected at a total throughput of $1300 \mathrm{~mL} / \mathrm{min}$. Entrainment in the aqueous sample increased when the throughput was increased to $1400 \mathrm{~mL} / \mathrm{min}$. Subsequently, when the rotor speed was increased to $4000 \mathrm{rpm}$, no effect on performance (i.e., no improvement) was observed. 
Additional testing was performed using straight lower vanes ( 8 radial vanes). Testing in this configuration was initiated at a phase ratio of 1:2 and a rotor speed of $3600 \mathrm{rpm}$. A slight film of organic was observed on an aqueous effluent sample at a total throughput of $1230 \mathrm{~mL} / \mathrm{min}$. Increasingly significant sheens were observed on samples collected at throughputs of 1290, 1350, and $1440 \mathrm{~mL} / \mathrm{min}$. Flows were reduced and reset to obtain a throughput O:A ratio of 1:3. From an initial throughput of $520 \mathrm{~mL} / \mathrm{min}$, flows were increased incrementally with no indication of entrainment up to a throughput of $960 \mathrm{~mL} / \mathrm{min}$. Sheens of organic material on aqueous effluent samples were observed at $1080 \mathrm{~mL} / \mathrm{min}(270: 810)$ and $1120 \mathrm{~mL} / \mathrm{min}$ (280:840). Desirable performance was reestablished by reducing the throughput to $960 \mathrm{~mL} / \mathrm{min}$ and was lost again upon increasing flow to $1080 \mathrm{~mL} / \mathrm{min}$. At the 1:4 phase ratio, no entrainment was observed up to a throughput of $900 \mathrm{~mL} / \mathrm{min}$. A solvent film was observed over aqueous effluent samples collected at 1000 and $1050 \mathrm{~mL} / \mathrm{min}$.

Testing was repeated using a heavy phase weir with a $0.95 \mathrm{in}$. heavy phase weir. At a phase ratio of 1:2 both aqueous and organic samples were transparent and free of cross-phase contamination up to a total throughput of $600 \mathrm{~mL} / \mathrm{min}$. At $600 \mathrm{~mL} / \mathrm{min}$ and a 1:3 phase ratio, contamination was not apparent but the aqueous effluent samples were no longer transparent. The same observation was made at a phase ratio of 1:4 and a throughput of $550 \mathrm{~mL} / \mathrm{min}$. At the 1:2 phase ratio, the first distinct appearance of an organic film on aqueous samples appeared at $1050 \mathrm{~mL} / \mathrm{min}$ (350:700). The first appearance of an organic film on aqueous samples collected at the 1:3 ratio occurred at $960 \mathrm{~mL} / \mathrm{min}$; aqueous sample contamination at 1:4 was also first observed at $900 \mathrm{~mL} / \mathrm{min}$. Flows were reset to 200:600 (O:A in $\mathrm{mL} / \mathrm{min}$ ), which resulted in elimination of visible carryover. Droplets of solvent in aqueous samples reappeared upon increase of the flow rate to $960 \mathrm{~mL} / \mathrm{min}$. Increasing the rotor speed to $4200 \mathrm{rpm}$ had no visible effect on entrainment. Still at the 1:3 phase ratio, flows were reset to $900 \mathrm{~mL} / \mathrm{min}$ total and acceptable operation (at $4200 \mathrm{rpm}$ ) was restored. The rotor speed was reduced to $3600 \mathrm{rpm}$ with no change in sample quality. Contamination of the aqueous effluent sample reappeared upon increasing the flow rate to $1000 \mathrm{~mL} / \mathrm{min}$ at the $1: 3$ phase ratio. Additional testing was performed at $3600 \mathrm{rpm}$ and the $1: 3$ ratio at throughputs up to $1520 \mathrm{~mL} / \mathrm{min}$. While some increase in the number of organic droplets in the aqueous phase was observed, catastrophic failure (i.e., appearance of large quantities of the dispersed phase) was never seen. At no point in all extraction-condition testing performed was contamination of the solvent with aqueous solution indicated.

\subsubsection{Scrubbing}

Initial testing was performed at the current baseline phase ratio of 5:1, using a contactor operated at 3600 rpm that was equipped with a 1.0 in. heavy phase weir. As stated previously, the scrub solution used was 
$25 \mathrm{mM} \mathrm{NaOH}$; solvent used had been recovered from hydraulic testing performed under extraction conditions, and had therefore been equilibrated with waste simulant.

No entrainment was observed in samples of either effluent at a total throughput of $600 \mathrm{~mL} / \mathrm{min}$. The onset of carryover of organic into the aqueous effluent was seen at $660 \mathrm{~mL} / \mathrm{min}$ in the form of a sheen on the aqueous sample surface. Flows were then lowered and reset to an approximate phase ratio of 3.75:1. (The pumps used are adjustable in increments of $10 \mathrm{~mL} / \mathrm{min}$, limiting the ability to create the precise phase ratio with small variations in total flow.) Contactor conditions (speed and weir size) were unchanged from the previous test. At this phase ratio, droplets of solvent were visible at the upper surface of aqueous effluent samples at a throughput of $560 \mathrm{~mL} / \mathrm{min}$. Solvent flow was terminated and the aqueous flow rate was increased to $700 \mathrm{~mL} / \mathrm{min}$ to clear the collector ring and discharge tubing of solvent. The scrub solution flow was decreased to $100 \mathrm{~mL} / \mathrm{min}$ and solvent flow was resumed at $200 \mathrm{~mL} / \mathrm{min}$. A $25-\mathrm{mL}$ sample of aqueous effluent was collected and found to be free of solvent when examined under a magnifying glass. Additional samples were collected after 5, 7, 9, 11, and 15 minutes of operation. Slightbut-increasing levels of organic contamination in the aqueous effluent were observed. The solvent flow rate was increased to $300 \mathrm{~mL} / \mathrm{min}$ (for an O:A ratio of 3:1). After 2 minutes of operation at this condition, solvent droplets were observed in aqueous effluent samples. Additional samples were collected after 4 and 8 minutes of operation; increasing levels of solvent carryover with increased period of operation were observed. Solvent flow was increased to $370 \mathrm{~mL} / \mathrm{min}$, which resulted in an increased number of solvent droplets in the aqueous effluent. Additional increases in flow were made to 560 and $660 \mathrm{~mL} / \mathrm{min}$ total throughput (at a constant 3.75:1 phase ratio), both resulting in increased aqueous effluent contamination.

Under no conditions was contamination of the organic effluent with aqueous solution indicated.

\subsubsection{Stripping}

Testing under the stripping condition was performed using a rotor fitted with a 1.0 in heavy phase weir, operating at $3600 \mathrm{rpm}$. The strip solution used was $10 \mathrm{mM} \mathrm{H}_{3} \mathrm{BO}_{3}$; solvent used was the solution used previously (and sequentially) in extraction- and scrubbing-condition hydraulic testing.

Testing was performed at phase ratios of 3:1, 3.75:1, 4:1, and 5:1. At all phase ratios, excellent phase separation was achieved at throughputs up to $500 \mathrm{~mL} / \mathrm{min}$. At the $5: 1$ phase ratio, contamination of aqueous effluent samples was first indicated at $600 \mathrm{~mL} / \mathrm{min}$. Aqueous sample contamination was observed at $630 \mathrm{~mL} / \mathrm{min}$ at $3.75: 1$, and at $600 \mathrm{~mL} / \mathrm{min}$ at the $4: 1$ phase ratio. Aqueous samples collected under these conditions were transparent, i.e., there was no visible indication of entrained organic as 
dispersed 'haze.' Testing was continued to total throughputs of $1250 \mathrm{~mL} / \mathrm{min}$ (at 4:1), $1500 \mathrm{~mL} / \mathrm{min}$ (at $5: 1)$ and $1660 \mathrm{~mL} / \mathrm{min}(3.6: 1)$. At these flow rates there was the continued appearance of organic droplets in aqueous effluent samples (but no organic layer) and increased turbidity of the aqueous effluent.

There was no indication of aqueous carryover in organic effluent samples at any of the conditions examined.

\subsubsection{Washing}

Due to the need to proceed with mass transfer testing and because phase separation evaluations under washing conditions are not needed to support mass transfer evaluations, no hydraulic testing under solvent washing conditions was performed.

\subsubsection{Summary of Results}

Defining acceptable performance as a carryover rate that can be addressed with downstream coalescers, use of the new solvent formulation poses no limitations relative to the baseline BOBCalixC6 CSSX blend. Physical modifications to the device had little effect on performance. Increased rotor speed, which should reduce entrainment due to inadequate dispersion separation, had no noticeable effect on organic carryover in aqueous effluents. Similarly, changing the heavy phase weir diameter, which shifts the position of the dispersion band within the rotor, had no significant effect on contactor throughput in the testing that was performed.

Under extraction conditions, changing the phase ratio from 1:3 to 1:4 in order to increase waste processing capacity has a slight adverse effect on throughput, but the impact is not great enough to negate the benefit of changing the ratio.

Throughput under scrubbing and stripping conditions was favorable at throughputs less than $600 \mathrm{~mL} / \mathrm{min}$ at phase ratios ranging from 3.75:1 to 5:1, though separation under scrubbing conditions was not as effective as under stripping.

Increases in organic carryover into aqueous effluent as a function of time, as was indicated during scrubcondition testing, do not indicate poor separation within the rotor. If separation within the rotor is poor resulting in a contaminated rotor effluent, the effluent exiting the contactor should display a constant level of contamination. It is more likely that the time dependency on the level of organic contamination of the 
aqueous effluent reflects accumulation of organic in the aqueous discharge path resulting from some effect other than inadequate separation within the rotor.

\subsection{MASS TRANSFER EVALUATIONS}

Two sets of mass transfer experiments were performed, with a set defined as testing under extraction, scrubbing and stripping conditions. In each set, the organic product from extraction was collected and used in the subsequent scrub- and strip-condition tests.

Results from the first test were indicative of acceptable performance in extraction and poorer than-desired stripping performance. In both cases, the test conditions were sufficiently different from previous testing of BOBCalixC6 CSSX chemistry (performed a decade ago) to prevent direct comparison. In order to address an apparent limitation in stripping performance and to facilitate direct comparison between NGCSSX and BOBCalixC6 CSSX system performance, a second mass transfer test of the NG-CSSX chemistry was performed under conditions identical to the prior BOBCalixC6 CSSX work.

\subsubsection{Reagents Used}

The composition of solvent used in all mass transfer tests was listed previously (Table 10.9). The aqueous feed used in testing under extraction conditions was a simplified SRS waste simulant, described in Table 10.11. The aqueous scrub solution used in testing was $25 \mathrm{mM} \mathrm{NaOH} ; 10 \mathrm{mM} \mathrm{H} \mathrm{BO}_{3}$ was the strip solution. The aqueous solutions were prepared using at least ACS reagent grade chemicals, which were dissolved or diluted with deionized water.

The simple simulant was spiked with $0.1 \mathrm{mCi}$ of $\mathrm{Cs}-137$, which allows the samples to be counted by a gamma detector. The previous BOBCalixC6 CSSX work did not use Cs-137, but instead used ICP/atomic emission spectroscopy to determine cesium concentration ${ }^{27}$. The 'cold' chemical analysis has the distinct disadvantage of low resolution for $\mathrm{Cs}$, resulting in potentially large error at low concentrations. By using the Cs-137 tracer, the sample processing time was reduced from 2 weeks to less than one day and the error was reduced from $10 \%$ to less than $1 \%$.

Table 10.11. Simple Savannah River simulant formulation

\begin{tabular}{lc}
\hline \multicolumn{1}{c}{ Chemical } & Concentration M \\
\hline Sodium Nitrate $\left(\mathrm{NaNO}_{3}\right)$ & 1.17 \\
Potassium Nitrate $\left(\mathrm{KNO}_{3}\right)$ & 0.015 \\
Cesium Nitrate $\left(\mathrm{CsNO}_{3}\right)$ & 0.00014 \\
Sodium Sulfate $\left(\mathrm{Na}_{2} \mathrm{SO}_{4}\right)$ & 0.14
\end{tabular}


Table 10.11. Simple Savannah River simulant formulation

\begin{tabular}{lc}
\hline \multicolumn{1}{c}{ Chemical } & Concentration M \\
\hline Sodium Hydroxide $(\mathrm{NaOH})$ & 3.18 \\
Aluminum Nitrate $\left(\mathrm{Al}\left(\mathrm{NO}_{3}\right)_{3}\right)$ & 0.280 \\
Sodium Carbonate $\left(\mathrm{Na}_{2} \mathrm{CO}_{3}\right)$ & 0.15 \\
Sodium Chloride $(\mathrm{NaCl})$ & 0.024 \\
Sodium Nitrite $\left(\mathrm{NaNO}_{2}\right)$ & 0.64 \\
\hline
\end{tabular}

\subsubsection{Mass Transfer Test Procedure-Initial Test}

Mass transfer testing was performed in a CINC Model V-2 centrifugal contactor identical to the unit used in hydraulic testing. The device was fitted with an end cap having 8 straight, radial vanes to maximize mixing. Pumps used were also identical to those used in hydraulic testing. However, the test equipment was configured for once through processing of all solutions. Under all test conditions, the contactor was operated at a speed of $3600 \mathrm{rpm}$ and was fitted with a heavy phase weir having a 1.0 in diameter opening.

Prior to all contactor mass transfer operations aliquots of the feed solutions were collected for determination of initial feed concentrations, which serve as a basis for stage efficiency calculations. In addition, the feed solution aliquots were contacted in the proportion equal to the phase ratio used in testing in order to obtain distribution coefficients. Dispersion of the phases was performed manually using the same agitation technique as was used in dispersion number determinations. Dispersions were separated by gravity; samples from the separated phases were collected using a manual pipettor.

In all tests, the aqueous solution (feed, scrub, or strip acid) was introduced alone until discharge of solution from the heavy phases collector ring was observed. Organic feed solution flow was initiated at that point. Timing of the experiment was initiated when the organic feed first entered the contactor mixing zone. All initial phase testing was performed using a standard, fully-pumping contactor rotor.

Extraction testing was performed at a phase ratio of 1:4 and a total flow rate of $600 \mathrm{~mL} / \mathrm{min}$. The flow rate was selected based on hydraulic testing results that indicated no discernible cross-phase contamination at this condition. Samples were collected from both discharge streams at 1, 2, 3, and 5 minutes after introduction of solvent.

Similarly, scrubbing was performed at a phase ratio of 3.77:1 and a total flow rate of $620 \mathrm{~mL} / \mathrm{min}(490$ $\mathrm{mL} / \mathrm{min}$ solvent: $130 \mathrm{~mL} / \mathrm{min} 25 \mathrm{mM} \mathrm{NaOH}$ ). Solvent used in this test was the collected effluent from the extraction-condition test. Due to the higher solvent flow rate and the limited solvent inventory available, samples were collected at $60 \mathrm{~s}, 90 \mathrm{~s}$, and $120 \mathrm{~s}$. 
Strip-condition testing was performed at a phase ratio of $5: 1$ and a total throughput of $600 \mathrm{~mL} / \mathrm{min}$. As stated previously, the strip solution used was $\mathrm{H}_{3} \mathrm{BO}_{3}$ at a concentration of $10 \mathrm{mM}$. Due to solvent inventory availability the test duration was brief; effluent samples were collected at $45 \mathrm{~s}, 60 \mathrm{~s}$, and $90 \mathrm{~s}$. The solvent supply became depleted at the time the last sample collection was completed.

\subsubsection{Results of Initial Mass Transfer Test}

Results of tests performed under all three conditions are presented in Table 10.12. The reported efficiencies were calculated using the Murphree definition based on aqueous-phase concentrations for extraction and organic-phase concentrations for stripping. Efficiencies have not been calculated for the scrubbing condition as the transfer of cesium between phases in the scrubbing section of a BOBCalixC6 CSSX cascade is not of primary interest. The Murphree efficiency calculated under the extraction condition is defined for a single stage by Eq. $1 ; X$ denotes aqueous phase concentration and the subscript $f$ indicates feed solution.

$$
\eta=\frac{X_{f}-X_{\text {aqueous stage effluent }}}{X_{f}-X_{\text {aqueous equilibration sample }}}
$$

Table 10.12. Results of first mass transfer test

\begin{tabular}{|c|c|c|c|c|c|}
\hline Condition & Elapsed time & $\begin{array}{c}\text { organic } \\
\text { sample, } \\
\text { counts } / \mathbf{m L}\end{array}$ & $\begin{array}{c}\text { aqueous } \\
\text { sample, } \\
\text { counts/mL }\end{array}$ & $\boldsymbol{D}_{C s}$ & $\begin{array}{c}\text { Murphree } \\
\text { Efficiency\% }\end{array}$ \\
\hline \multirow[t]{5}{*}{ Extraction } & $1 \mathrm{~min}$ & 157201 & 6304.5 & 24.93 & 92.3 \\
\hline & $2 \min$ & 153802 & 4873 & 31.56 & 95.8 \\
\hline & $3 \mathrm{~min}$ & 152223 & 4913.5 & 30.98 & 95.7 \\
\hline & $5 \mathrm{~min}$ & 86931 & 4784.5 & 18.17 & 96.0 \\
\hline & Equilibration & 164998 & 3168 & 52.08 & \\
\hline \multirow[t]{4}{*}{ Scrub } & $60 \mathrm{~s}$ & 107397 & 20333 & 5.28 & \\
\hline & $90 \mathrm{~s}$ & 108111 & 36576.5 & 2.96 & \\
\hline & $120 \mathrm{~s}$ & 109128 & 46239.5 & 2.36 & \\
\hline & Equilibration & 118398 & 30474 & 3.89 & \\
\hline \multirow[t]{4}{*}{ Strip } & $45 \mathrm{~s}$ & 84945 & 12173 & 6.98 & 40.6 \\
\hline & $60 \mathrm{~s}$ & 84647.5 & 154505 & 0.55 & 41.1 \\
\hline & $90 \mathrm{~s}$ & 86009 & 108020 & 0.80 & 38.9 \\
\hline & Equilibration & 49001.5 & 312855 & 0.16 & \\
\hline
\end{tabular}

Efficiencies under the extraction condition are uniformly high and are typical for centrifugal contactor performance. Mass transfer efficiencies under the stripping condition (calculated from organic phase results) were low, a result which was also obtained during initial stripping tests performed in 2001 using 
the BOBCalixC6 CSSX process chemistry. The validity of the stripping results can be called into question, given the high degree of variation in the corresponding aqueous-phase results.

\subsubsection{Mass Transfer Test Procedure-Second Test}

A single contactor stage was configured for once-through processing and all testing was performed at a rotor speed of $3600 \mathrm{rpm}$, as before. Unlike the initial test described above, the flow rates and phase ratios utilized in this test were the same as had been used in previous mass transfer tests on the BOBCalixC6 CSSX solvent. This testing scheme was intended to allow for the direct comparison of results with the results of tests performed in $2001^{27}$. In addition, a partially pumping contactor rotor was used. (A partially pumping rotor is one in which the lower opening has been increased in size such that the remaining lower surface is insufficient to support a liquid cylinder of sufficient height to reach the weir diameter. Consequently, lifting of liquid is partially dependent on hydraulic head in the mixing zone, resulting in partial flooding, increased liquid holdup, and increased residence time in the mixing zone of the contactor.)

Before each test, the contactor was rinsed with deionized water and then drained. As in all previous testing, the flow of aqueous solution was initiated with the rotor turning at $3600 \mathrm{rpm}$. Organic feed solution flow was initiated when aqueous solution was observed flowing from the aqueous discharge. The experiment clock was started once the organic entered the contactor. Stage samples were collected at various times once the experimental clock was started. To maximize test duration, contactor operation was continued until the organic feed solution was depleted. At this point, the aqueous feed flow was continued until flow from the organic discharge ceased. The rotor was left spinning until the aqueous solution stopped flowing from the discharge. Then, the rotor was stopped and the remaining solution was drained through a valve at the bottom of the contactor housing. As before, all organic solution from a phase of testing was collected from the previous test to for use in the subsequent test phase. The scrub and strip solvent feeds included the solvent effluent collected during the startup and shutdown processes from the previous phase of testing.

During each mass transfer test, samples of the organic and aqueous effluent were collected and stored in glass vials. Sample drains were installed on the discharge lines to prevent sample spillage. The drains were 0.375 -in OD tubing (0.062-in wall) and were approximately 2 inches long from valve to contactor discharge line (the reservoir); holdup of liquid in the sample drains was approximately $0.5 \mathrm{~mL}$. The drains were flushed for a minimum of $15 \mathrm{~s}$ before samples for analysis were collected, during which $>20 \mathrm{~mL}$ of solution was collected. Approximately $10 \mathrm{~mL}$ of solution was collected during each sampling period. In 
addition to the samples collected during the mass transfer tests, samples of the feed solutions were also collected and processed as in the previous tests. For consistency, contactor mass transfer testing was performed immediately after the equilibration of feed solutions.

The reported gamma counts have been corrected for background via subtraction.

\subsubsection{Results of Second Mass Transfer Test}

\subsubsection{Extraction}

During the extraction test, samples of aqueous and organic effluents were collected after $4 \mathrm{~min}, 7 \mathrm{~min}$, and $10 \mathrm{~min}$ of operation. The samples were allowed to sit for 72 hours and no cross-phase contamination was observed. The aqueous flow rate was $480 \mathrm{~mL} / \mathrm{min}$ while the organic flow rate was $150 \mathrm{~mL} / \mathrm{min}$. The weir size used for the extraction tests was 1.000 in. A $1 \mathrm{~mL}$ aliquot was taken from each sample for gamma counting for $30 \mathrm{~min}$ to determine the amount of cesium in the organic and aqueous solutions. Using these results (Table 10.13) and the results from the feed (Table 10.14) and shakeout samples (Table 10.15), the stage efficiencies were calculated.

Stage efficiencies obtained are marginally higher than those obtained previously, remaining consistently higher than is required to meet target MCU DFs.

\subsubsection{Scrub}

All of the solvent from the extraction test was collected and became the feed for the scrub test. The solvent went through two scrub tests to ensure the potassium was removed from the solvent to condition the solution for subsequent stripping. For each scrub test, the aqueous flow rate was $30 \mathrm{~mL} / \mathrm{min}$ and the organic flow was $150 \mathrm{~mL} / \mathrm{min}$. Originally, a heavy phase weir having a 1.000 in.-dia. opening was used, but considerable aqueous carryover was observed in the organic effluent during the first scrub pass. The pumps and contactor were shut down and a replacement weir having a $1.125 \mathrm{in}$. opening was installed. The test was restarted, and organic was observed in the aqueous effluent. The weir was replaced again with a 1.075 in.-dia. Weir. Organic carryover was reduced but remained. Finally, a weir with a 1.025 in.dia. opening was installed, which provided adequate separation for the rest of the first scrub pass as well as the second pass. Liquid effluent samples of both phases were collected at $4 \mathrm{~min}, 7 \mathrm{~min}$, and $10 \mathrm{~min}$ of the second pass. Samples were not collected on the first scrub pass. All of the solvent was collected and became the organic feed for the strip test. 


\subsubsection{Strip}

The stripping test was performed at the same flow conditions as the scrub test. As in the scrub test, a 1.025-in. heavy phase weir was used; a small degree of aqueous carryover in the organic effluent was observed. However, the limited quantity of solvent feed available precluded replacement of the weir as insufficient feed was available for a restart without impacting test duration significantly. The inability to properly separate the solutions inside the rotor does not affect the mass transfer that takes place in the mixing zone and the aqueous carryover in the organic samples was sufficiently disengaged that uncontaminated organic aliquots could easily be recovered for gamma counting. Effluent samples were collected at $4 \mathrm{~min}, 7 \mathrm{~min}$, and $9.25 \mathrm{~min}$. Organic aliquots for counting were removed from the grab samples immediately after collection to reduce incidental mass transfer between the organic effluent and the aqueous carryover. Due to the low level of activity in the stripped solvent and administrative limits on how much Cs-137 that could be added as a counting spike, $2 \mathrm{~mL}$ organic samples were collected (as opposed to $1 \mathrm{~mL}$ samples for aqueous effluent) and were counted for 30 minutes.

Stripping stage efficiencies are markedly higher than those indicated from the initial test for NG-CSSX stripping chemistry, but remain lower than those obtained in extraction. Possible causes of the difference between extraction and stripping performance are the difference in phase ratio, difference in dispersed versus continuous phase, and differences in kinetics between extraction and stripping processes. While stripping efficiency does not match extraction efficiency, it is still high enough to effectively recover cesium from the solvent in the MCU flowsheet.

Table 10.13. NG-CSSX mass transfer efficiency

\begin{tabular}{|c|c|c|c|c|c|c|c|}
\hline $\begin{array}{c}\text { Test } \\
\text { Condition }\end{array}$ & $\begin{array}{c}\text { Sample } \\
\text { Collection } \\
\text { Time } \\
\text { (min) }\end{array}$ & $\begin{array}{c}\text { Aqueous } \\
\text { Flow } \\
(\mathrm{mL} / \mathrm{min})\end{array}$ & $\begin{array}{c}\text { Organic } \\
\text { Flow } \\
(\mathrm{mL} / \mathrm{min}) \\
\end{array}$ & $\begin{array}{c}\text { Aqueous Effluent } \\
\text { Cs Conc. } \\
\text { (counts/mL) }\end{array}$ & $\begin{array}{c}\text { Organic Effluent } \\
\text { Cs Conc. } \\
\text { (counts/mL) }\end{array}$ & $D_{C s}$ & $\begin{array}{c}\text { Efficiency } \\
(\%)\end{array}$ \\
\hline Extraction & 4 & 480 & 150 & 1613 & 91300 & 56.6 & 98.8 \\
\hline Extraction & 7 & 480 & 150 & 1378 & 90236 & 65.5 & 99.6 \\
\hline Extraction & 10 & 480 & 150 & 1325 & 90488 & 68.3 & 99.8 \\
\hline Scrub & 4 & 30 & 150 & 14687 & 94848 & 6.5 & 24.5 \\
\hline Scrub & 7 & 30 & 150 & 22206 & 92515 & 4.2 & 41.4 \\
\hline Scrub & 10 & 30 & 150 & 26500 & 91423 & 3.5 & 49.3 \\
\hline Strip & 4 & 30 & 150 & 303391 & 41885 & 0.1 & 72.2 \\
\hline Strip & 7 & 30 & 150 & 334385 & 39636 & 0.1 & 75.5 \\
\hline Strip & 9.25 & 30 & 150 & 335339 & 40831 & 0.1 & 73.8 \\
\hline
\end{tabular}

Table 10.14. NG-CSSX feed results

\begin{tabular}{ccc}
\hline & $\begin{array}{c}\text { Aqueous Cs Conc. } \\
\text { Test Condition }\end{array}$ & $\begin{array}{c}\text { Organic Cs Conc. } \\
\text { (counts } / \mathbf{m L})\end{array}$ \\
\hline
\end{tabular}




\begin{tabular}{ll}
\hline Extraction & 309450 \\
Scrub $\left(2^{\text {nd }}\right.$ Pass $)$ & \\
Strip & 98225 \\
\hline
\end{tabular}

Table 10.15. NG-CSSX equilibration results

\begin{tabular}{lccccc}
\hline $\begin{array}{c}\text { Test } \\
\text { Condition }\end{array}$ & $\begin{array}{c}\text { Aqueous } \\
(\mathbf{m L})\end{array}$ & $\begin{array}{c}\text { Organic } \\
(\mathbf{m L})\end{array}$ & $\begin{array}{c}\text { Aqueous Cs Conc. } \\
\text { (counts/mL) }\end{array}$ & $\begin{array}{c}\text { Organic Cs } \\
\text { Conc. } \\
\text { (counts/mL) }\end{array}$ & $\boldsymbol{D}_{\boldsymbol{C} \text { s }}$ \\
\hline Extraction & 480 & 150 & 1270 & 94366 & 74.3 \\
Scrub & 30 & 150 & 61282 & 84431 & 1.4 \\
Strip & 30 & 150 & 347395 & 22656 & 0.07 \\
\hline
\end{tabular}

Table 10.16. BOBCalixC6 CSSX mass transfer efficiencies - extraction ${ }^{1}$

\begin{tabular}{lcccccccc}
\hline $\begin{array}{c}\text { Aqueous } \\
\text { Flow } \\
(\mathbf{m L} / \mathbf{m i n})\end{array}$ & $\begin{array}{c}\text { Organic } \\
\text { Flow } \\
(\mathbf{m L} / \mathbf{m i n})\end{array}$ & $\begin{array}{c}\text { Rotor } \\
\text { Speed } \\
(\mathbf{r p m})\end{array}$ & $\begin{array}{c}\text { Stage } \\
\text { Temperature } \\
\left({ }^{\circ} \mathbf{C}\right)\end{array}$ & $\begin{array}{c}\text { Aqueous } \\
\text { Effluent Cs } \\
\text { Conc. }(\mathbf{p p m})\end{array}$ & $\begin{array}{c}\text { Organic } \\
\text { Effluent Cs } \\
\text { Conc. (ppm) }\end{array}$ & $\begin{array}{c}\text { Equilibrated } \\
\text { Organic Phase } \\
\text { Cs. Conc. (ppm) }\end{array}$ & $\mathbf{D}_{\text {Cs }}$ & Efficiency\% \\
\hline 484 & 150 & 3600 & 23.8 & 1.75 & 39.4 & 44.1 & 22.5 & 88.8 \\
484 & 150 & 3600 & 23.8 & 1.83 & 38.8 & 44.7 & 21.2 & 86.1 \\
306 & 95 & 3600 & 23.5 & 1.52 & 40.3 & 44.4 & 26.5 & 90.3 \\
306 & 95 & 3600 & 23.5 & 1.54 & 39.7 & 44.9 & 25.8 & 87.8 \\
\hline
\end{tabular}

Table 10.17. ВOBCalixC6 CSSX mass transfer efficiencies - strip ${ }^{1}$

\begin{tabular}{|c|c|c|c|c|c|c|c|c|}
\hline $\begin{array}{c}\text { Aqueous } \\
\text { Flow } \\
(\mathrm{mL} / \mathrm{min})\end{array}$ & $\begin{array}{c}\text { Organic } \\
\text { Flow } \\
(\mathrm{mL} / \mathrm{min})\end{array}$ & $\begin{array}{l}\text { Rotor } \\
\text { Speed } \\
(\text { rpm) }\end{array}$ & $\begin{array}{c}\text { Stage } \\
\text { Temperature } \\
\left({ }^{\circ} \mathrm{C}\right) \\
\end{array}$ & $\begin{array}{c}\text { Aqueous } \\
\text { Effluent Cs } \\
\text { Conc. (ppm) } \\
\end{array}$ & $\begin{array}{c}\text { Organic } \\
\text { Effluent Cs } \\
\text { Conc. (ppm) } \\
\end{array}$ & $\begin{array}{c}\text { Equilibrated } \\
\text { Organic Phase } \\
\text { Cs. Conc. (ppm) }\end{array}$ & $\boldsymbol{D}_{C s}$ & Efficiency \% \\
\hline 30 & 150 & 3600 & 23.0 & 45.5 & 15.1 & 15.2 & 0.33 & 100.7 \\
\hline 30 & 150 & 3600 & 23.0 & 46.3 & 15.0 & 14.1 & 0.32 & 94.2 \\
\hline 60 & 300 & 3600 & 23.3 & 46.0 & 18.7 & 15.0 & 0.41 & 74.8 \\
\hline 60 & 300 & 3600 & 23. & 49.1 & 18.3 & 15.4 & 0.37 & 79.8 \\
\hline
\end{tabular}

\subsubsection{Summary}

Extraction stage efficiencies are consistently high; some increase is apparent in the second test. The only significant differences between first and second extraction tests are the use of the partially pumping rotor and a reduction in volumetric phase ratio (1:4 versus 1:3.2 organic-to-aqueous) in the latter test. Efficiencies are considerably higher than those obtained using BOBCalixC6 CSSX chemistry under the same test conditions (Table 10.16).

Stripping results are adequate for MCU implementation of NG-CSSX, but are lower than those obtained for stripping under the same conditions utilizing BOBCalixC6 CSSX chemistry (as indicated by comparison of Table 10.13 with Table 10.17). Efficiencies obtained for NG-CSSX are approximately equal to those obtained for BOBCalixC6 CSSX stripping at twice the throughput. Expressed differently, efficiencies for the two stripping processes are similar when the residence time in NG-CSSX is twice that 
in a BOBCalixC6 CSSX stripping operation. However (and as stated previously), stripping efficiencies are adequate for MCU implementation.

\subsection{EXAMINATION OF PHASE CARRYOVER}

\subsubsection{Preliminary testing}

Various test elements required operation of the contactor with a resident volume of liquid in the mixing zone but without flow of liquid through the device (a 'stagnant' condition). To achieve a no-flow liquid condition, contactor housings with bottom plates having no vanes were used. As described previously, operation of the contactor with this type of plate results in the formation of a vortex between the plate and the bottom surface of the rotor, which effectively prevents flow into the rotor and facilitates liquid holdup within the contactor mixing zone during 'no flow' operation.

Testing was performed to determine the volume of liquid required to achieve a significant liquid height in the mixing zone while avoiding pulsing of liquid from this region into the lower collector ring. In addition to using the modified bottom to promote vortex formation, internal vanes were removed from the rotor to prevent any liquid that entered the rotor from being lifted to either of the weirs. Testing to determine the volume of liquid required to achieve desired liquid heights under the no-flow condition was performed in a transparent housing that was built based on dimensions measured from a standard, stainless steel CINC model V-2 housing (Fig. 10.1). 


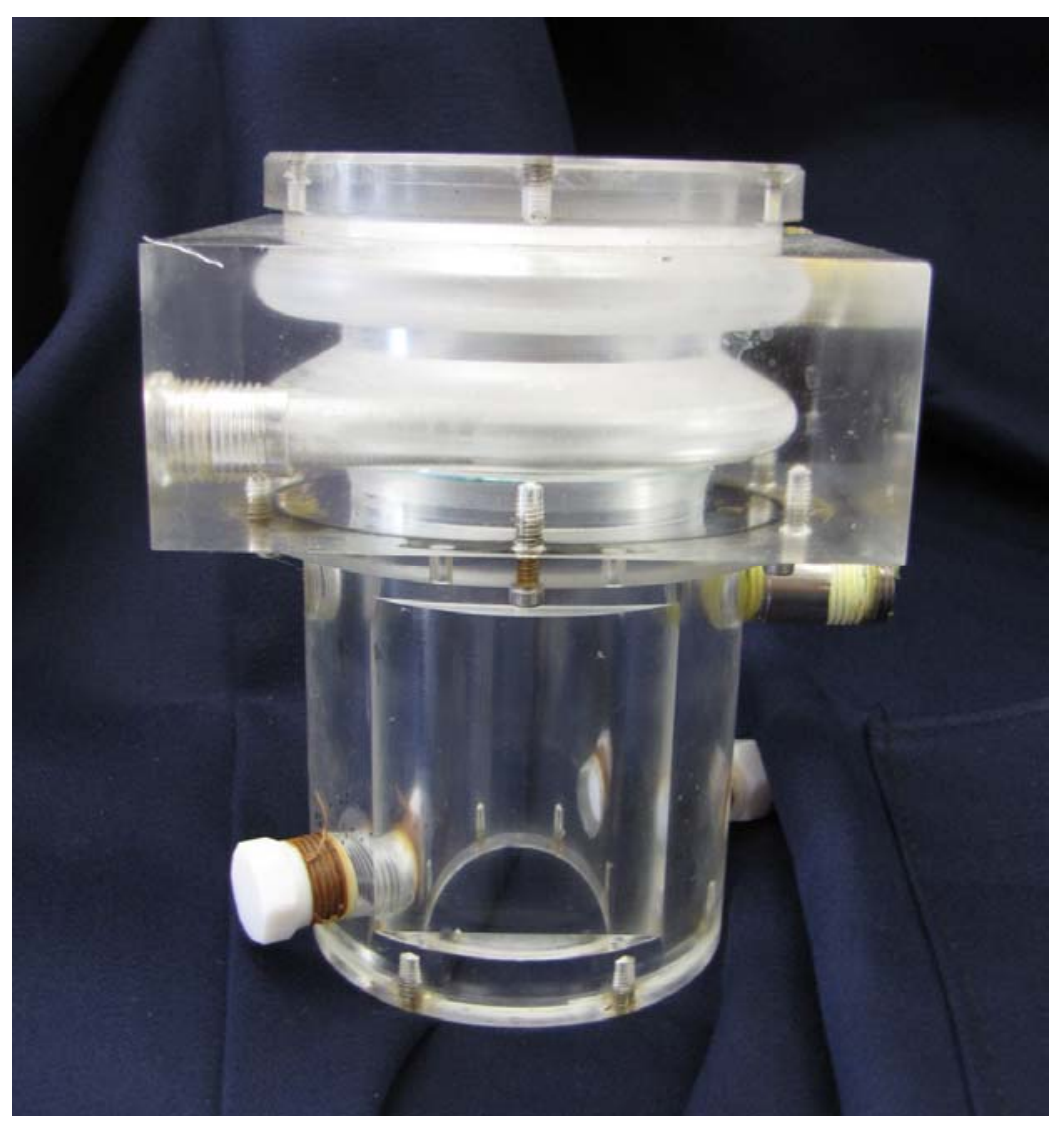

Fig. 10.1. Transparent housing based on CINC model V-2 contactor.

With modifications in place and no liquid in the system, contactor rotation was initiated at $3600 \mathrm{rpm}$. Liquid was added to the device from a graduated cylinder via one of the feed nozzles; the other nozzle was capped. The amount of liquid required to obtain a liquid seal at the bottom of the rotor, i.e., the volume required to fill the gap between the rotor and lower housing flange, (in a no-flow condition, recognizing that part of this volume is occupied by the vortex) was $19 \mathrm{~mL}$. The rotor speed was then increased to $4000 \mathrm{rpm}$ to determine if the seal remained, which it did.

The rotor speed was reset to $3600 \mathrm{rpm}$ and additional liquid was added until the liquid height in the mixing zone at maximum pulse height reached the bottom of the inlet nozzle. Obtaining this mixing zone height was found to require addition of $19 \mathrm{~mL}$ of liquid to that required to form a bottom seal. Again, the rotor speed was increased to $4000 \mathrm{rpm}$ to determine the effect on liquid height. The increase in rotor speed was observed in increase the maximum pulse height to the approximate centerline of the feed nozzle. 
Again, the rotor speed was reduced to $3600 \mathrm{rpm}$ and additional liquid was poured into the housing until the pulse height reached the baffle (the reduced diameter lip) just below the lower collector ring (Fig. 10.2). The total volume of liquid required to achieve this condition was $68 \mathrm{~mL}$ (including all previous liquid additions). The rotor speed was then increased to $4000 \mathrm{rpm}$, which resulted in periodic pulsing of liquid into the lower collector ring.

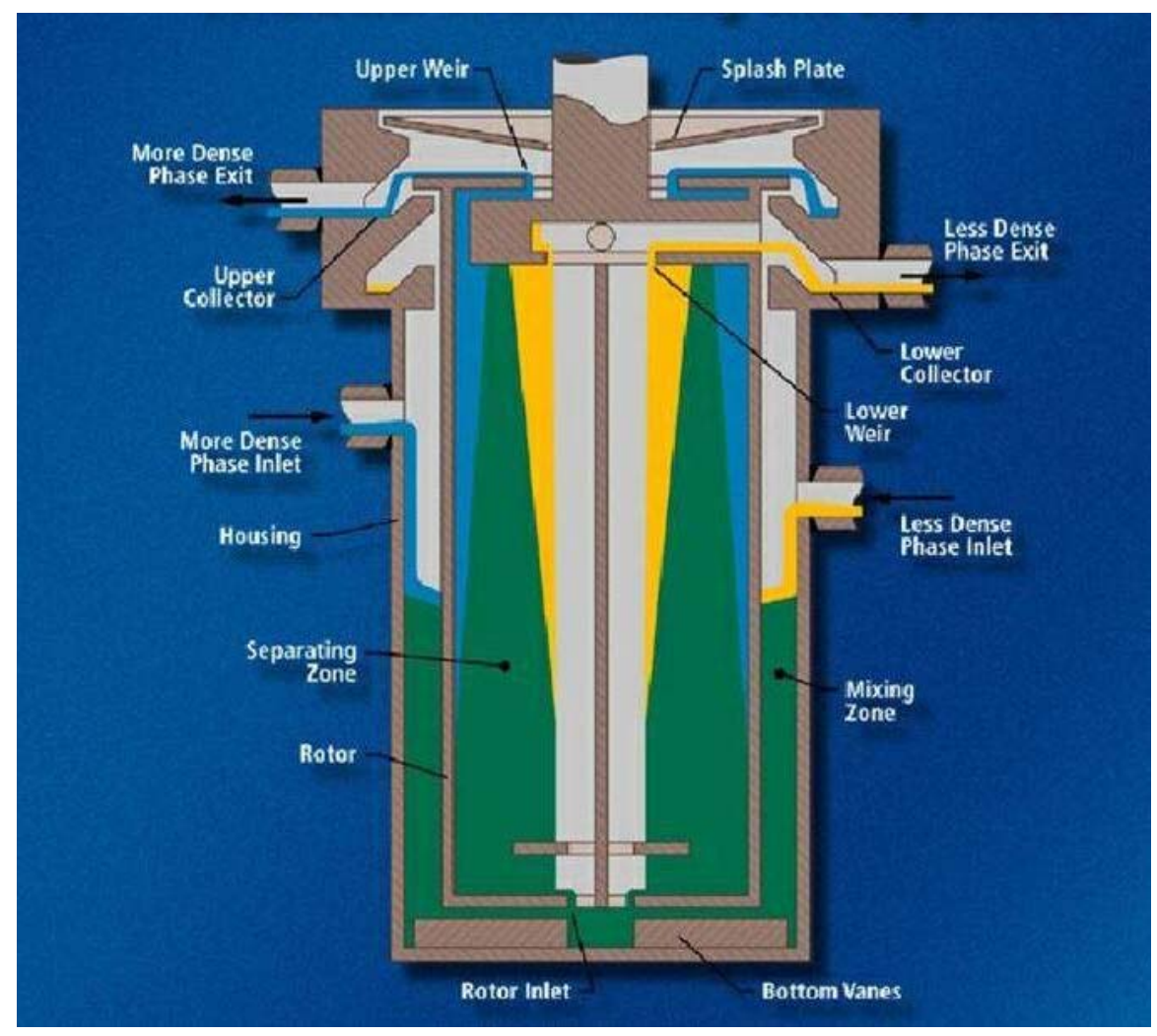

Fig. 10.2. Generic contactor flow path schematic.

\subsubsection{Identification of Entrainment Sources}

The first phase of testing performed (beyond hold-up determinations) was examination of the possible transfer of solution from the mixing zone into the collector ring region. Since the light phase collector ring (typically collecting solvent) is nearer the mixing zone than the heavy phase ring, transfer from the mixing zone would have the effect of preferentially contaminating the less dense effluent.

Using the volume measurements from the preceding test and retaining the same contactor configuration (i.e., the 'no flow' case), testing was performed to attempt to generate transfer of liquid from the mixing zone directly into the collector rings. However, to eliminate contactor materials of construction and any 
dimensional inconsistencies between the as-received CINC model V-2 contactor housing and the transparent replica, this phase of testing was performed in the former.

All contactor components were thoroughly dried prior to assembly. After assembly, contactor operation was initiated at $4000 \mathrm{rpm}$. A volume of NG-CSSX solvent simulant (solvent without MaxCalix) sufficient to produce a pulse that reached the feed nozzle $(38 \mathrm{~mL})$ was slowly added to the mixing zone. The contactor was operated for 30 minutes under this condition, after which the mixing zone was drained through an opening in the bottom housing plate without terminating contactor operation. Rotor speed was then slowly reduced to a complete stop. After stopping, the contactor was disassembled and the rotor was carefully removed from the housing so as not to allow liquid from the rotor surface to enter either collector ring.

After disassembly, both collector rings were wiped with foam tipped swabs that were then inspected for liquid. In addition, the rotor was removed from the bearing assembly, the cap over the heavy-phase weir was removed, and the upper and lower surfaces of the weir plate were examined for liquid to verify that no liquid was 'pumped' through the rotor.

After the period of operation under the conditions described, no traces of liquid were detected in either of the collector rings or on any of the rotor surfaces above the mixing zone of the contactor. Testing was not performed with aqueous solution. Due to its lower surface tension, the organic solvent is more susceptible to mist formation and represents a worst-case condition with respect to generation of droplet migration from the mixing zone into the region of the collector rings.

\subsubsection{Investigation of Rotor Discharge Behavior}

Testing to isolate any source(s) of entrainment in the upper region of the contactor - the rotor discharges and the collector rings - was performed using a CINC model V-2 contactor configured for continuous recycle using a single liquid feed and a single discharge stream. All testing was performed using a bottom housing plate with 8 straight, radial vanes. Solutions were transferred using the same model of pump used in previous hydraulic and mass transfer testing.

Initial testing was performed using water to which had been added dye to improve visibility. Testing was performed using a rotor insert having vanes that extend across the entire diameter of the rotor. All contactor components were thoroughly dried prior to assembly to avoid false indications of mist or droplet migration. 
With the contactor rotating at $3600 \mathrm{rpm}$, aqueous feed was initiated at a rate of $300 \mathrm{~mL} / \mathrm{min}$. Operation was continued for 10 minutes, timed from the first appearance of liquid in the contactor discharge line. At the end of the 10-min period, the feed pump was turned off and the contactor mixing zone was drained with the rotor still turning. After draining the mixing zone, contactor operation was terminated, allowing the rotor to drain. The contactor was disassembled and the rotor was removed from the housing taking care not to allow liquid from the rotor surface to enter the lower (light phase) collector ring. The lower collector ring was swabbed with a paper lab wipe that was compressed to fit inside the collector ring and pushed along the circumference of the trough. No liquid was observed, a fact that was confirmed by the lack of a mass increase of the swab material after wiping the collector ring. Subsequently, the contactor was reassembled and testing was repeated at a flow rate of $500 \mathrm{~mL} / \mathrm{min}$. After 10 minutes of operation the contactor was disassembled and inspected as before. No aqueous material was found in the light phase collector ring. Finally, the test was repeated at $500 \mathrm{~mL} / \mathrm{min}$ after increasing the rotor speed to $4200 \mathrm{rpm}$. As under the previous conditions, no carryover into the light phase collector ring was detected.

The contactor was disassembled, dried, and reassembled. The rotor was modified to force solvent into the light phase collector ring in the absence of an aqueous feed, since under normal operation, a single feed solution will pass from the rotor through the heavy phase underflows and into the heavy phase collector ring. The modification consisted of installing an aqueous weir plate with a small diameter (0.800 in) and placing a butyl rubber washer over the rotor shaft so as to cover the opening between the shaft and the heavy phase weir. In the test assembly created, the nut that holds the bearing pack in place on the rotor shaft also serves to compress the weir cap against the washer, sealing the heavy phase flow path.

To verify the effectiveness of the flow path modification, the contactor was operated for ten minutes after charging only enough liquid to the contactor to transfer some into the rotor, but not enough to cross either weir $(50 \mathrm{~mL})$. No liquid was observed in the area of the aqueous weir plate, i.e., no leakage from outer the circumference of the aqueous weir plate or across the washer used to block the aqueous weir was indicated.

The feed lines were drained and the aqueous solution (water) was replaced with simulated NG-CSSX solvent. With the contactor configured as before (straight bottom vanes, full diameter rotor vanes, and continuous liquid recycle), testing was performed at a rotor speed of $3600 \mathrm{rpm}$ and a flow rate of 100 $\mathrm{mL} / \mathrm{min}$. Upon disassembly, no visible indications of liquid were observed on any upper contactor surfaces. Mass measurements were not made since the flow rate condition is too low to maintain efficient 
mixing in the contactor, and would not normally be used as an operating condition. The upper regions of the contactor cylinder were wiped to remove any liquid that might have been present, and the unit was reassembled.

Subsequently, a test was conducted at a flow rate of $300 \mathrm{~mL} / \mathrm{min}$ and $3600 \mathrm{rpm}$ using the same equipment configuration as in the previous test. As before, the contactor was operated for 10 minutes recorded from the time liquid was first observed exiting the discharge nozzle. After 10 minutes, the feed pump was turned off and the contactor mixing zone was drained. Contactor rotation was terminated with the drain valve still open, allowing the contents of the device to drain without any increase in liquid height inside the apparatus. The unit was disassembled, with the rotor removed carefully to prevent any transfer of liquid from the cylindrical wall of the rotor into either collector ring. The upper (heavy-phase) collector ring was swabbed with a paper wipe that was folded to fit inside. The paper was weighed prior to use, using a calibrated scale with resolution to $0.1 \mathrm{mg}$. Weighing of the swab after testing indicated collection of $348.3 \mathrm{mg}$ of solvent in the upper collector ring. Two replicate tests were performed, after which 230.3 and $223.2 \mathrm{mg}$ of solvent were collected. In the replicate tests the collector ring was swabbed twice to absorb all the liquid present relative to the mass and surface area of the swabs. The contactor was disassembled after all tests and inspected to verify that liquid was not leaking past the heavy phase weir plate; in all cases the weir plate and washer surfaces were dry after testing.

On the assumption that reduced rotor vane surface in the vicinity of the rotor centerline could reduce air flow through the contactor, thereby mitigating droplet migration in the area of collector rings, testing was performed using a rotor insert on which the vanes extend inward only to the light (organic) phase weir.

After reassembling the contactor (with flow path modifications), testing was performed under the same flow and rotor speed conditions as before $-300 \mathrm{~mL} / \mathrm{min}$ flow at a rotor speed of $3600 \mathrm{rpm}$. The period of operation and the method of liquid collection after testing were the same as before. Testing under this set of conditions resulted in diversion of $279.1 \mathrm{mg}$ solvent into the upper collector ring. Replicate testing under these conditions resulted in collection of $320.5 \mathrm{mg}$.

Results obtained after changing the internal vane configuration indicate no consistent or significant impact on carryover.

Using the worst-case vane configuration, additional testing was performed to evaluated effects of increased flow rate and rotor speed on organic migration. Using the blocked-weir rotor with full diameter 
internal vanes, testing was performed at a flow rate of $500 \mathrm{~mL} / \mathrm{min}$ and a rotor speed of $3600 \mathrm{rpm}$, and at a flow rate of $300 \mathrm{~mL} / \mathrm{min}$ and a rotor speed of $4200 \mathrm{rpm}$. Replicate tests were performed at each of the conditions. Results are summarized in Table 10.18, below. The results indicate considerable scatter. No reduction in transfer due to the change in rotor vane profile is indicated. There does appear to be a negative impact associated with increased flow rate; given the scatter within the data, speed is not conclusively indicated as having an impact on mist/droplet migration between collector rings.

Table 10.18. Results of solvent carryover evaluations

\begin{tabular}{llccc}
\hline $\begin{array}{l}\text { Vane } \\
\text { configuration }\end{array}$ & Housing type & $\begin{array}{l}\text { Flow rate, } \\
\text { mL/min }\end{array}$ & $\begin{array}{l}\text { Rotor speed, } \\
\text { rpm }\end{array}$ & $\begin{array}{l}\text { Mass of solvent collected } \\
\text { from aqueous ring, mg }\end{array}$ \\
\hline Full diameter & Standard V-2 & 300 & 3600 & 344.8 \\
Full diameter & Standard V-2 & 300 & 3600 & $230.3^{a}$ \\
Full diameter & Standard V-2 & 300 & 3600 & $223.2^{a}$ \\
Partial diameter & Standard V-2 & 300 & 3600 & 279.1 \\
Partial diameter & Standard V-2 & 300 & 3600 & $320.5^{a}$ \\
Full diameter & Standard V-2 & 500 & 3600 & $307^{1}$ \\
Full diameter & Standard V-2 & 500 & 3600 & $402.3^{a}$ \\
Full diameter & Standard V-2 & 300 & 4200 & $288.2^{a}$ \\
Full diameter & Standard V-2 & 300 & 4200 & $226.9^{a}$ \\
Full diameter & Modified V-2 & 300 & 3600 & 6.4 \\
Full diameter & Modified V-2 & 300 & 3600 & 12.7 \\
Full diameter & Modified V-2 & 300 & 3600 & 12.3 \\
Full diameter & Modified V-2 & 500 & 3600 & 14.6 \\
Full diameter & Modified V-2 & 500 & 3600 & 6.3 \\
Full diameter & Modified V-2 & 300 & 4200 & 14.4 \\
Full diameter & Modified V-2 & 300 & 4200 & \\
\hline \\
Sum of masses collected from two swabbing operations, which were required due to the quantity of liquid obtained from the \\
collector ring.
\end{tabular}

Having confirmed that transfer of less-dense material does, in fact, occur and having isolated the transfer to the region between the collector rings and the rotor discharge points, no additional testing was performed using the standard CINC V-2 housing to quantify effects of flow rate and rotor speed on the phenomena. Instead, a housing having a modified collector ring configuration was installed and tested. The housing used was fabricated by CINC from drawings and instructions provided by ORNL. The modifications were made to correct two discrepancies between the CINC contactor models and previous ANL/ORNL/SRTC designs: excessive $\left(>45^{\circ}\right)$ downward pitch on the upper faces of the collector rings and the use of horizontal discharge nozzles. The latter has been found to result in accumulation of foam, particularly in the organic flow path, that results from the inherent mixing of air with organic solvent during discharge from the rotor. The former is suspected to promote splash back of discharge liquid into the gap between the rotor and housing in the vicinity of the collector rings. The standard and modified housings are shown in Fig. 10.3. 


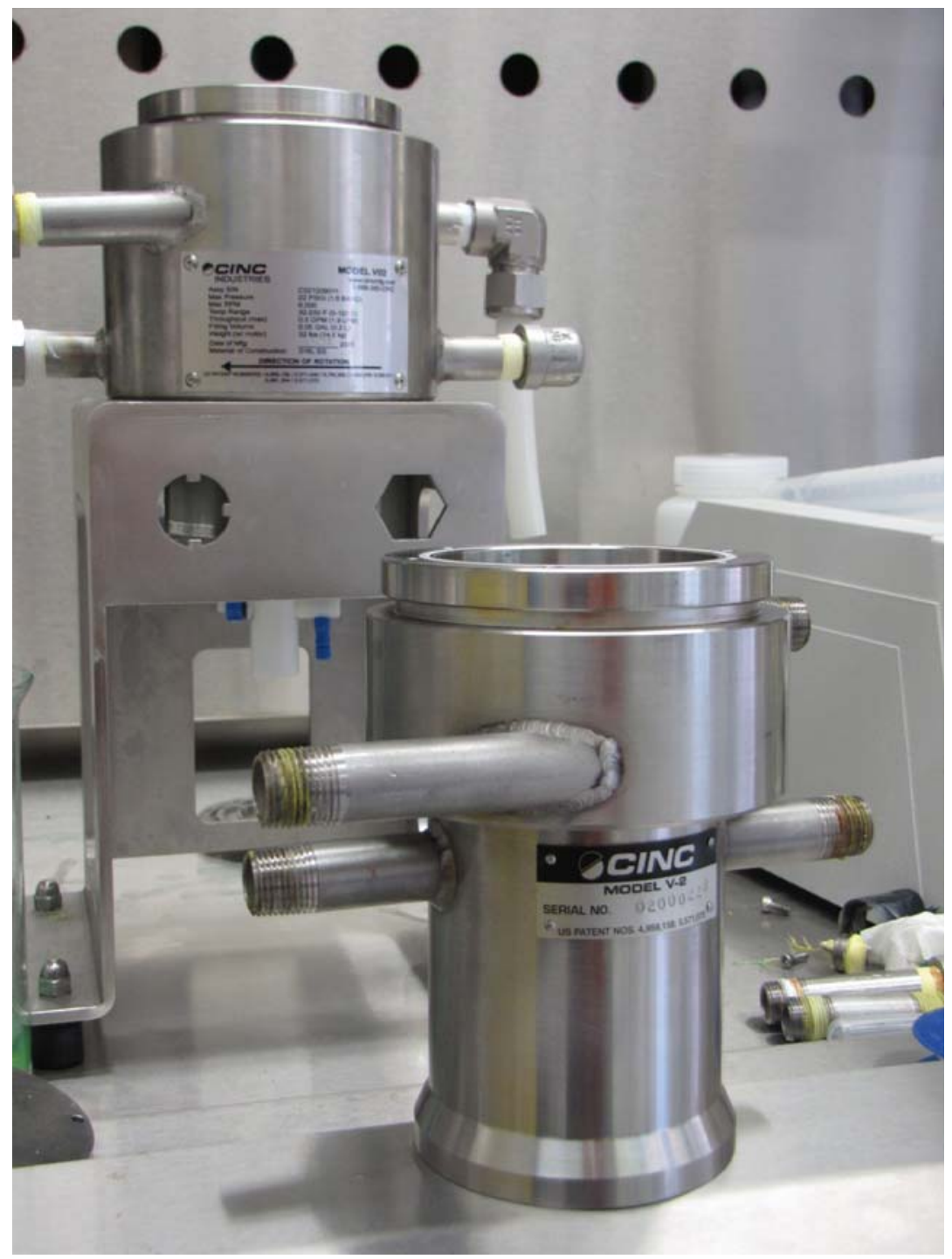

Fig. 10.3. Photograph of standard CINC V-2 housing (foreground) and modified housing.

Since there was no indication of aqueous (heavy) phase transfer into the light (organic) phase collector ring using a standard housing, testing using the modified housing was performed only for the organiconly flow case. In the initial case, the contactor was operated at $3600 \mathrm{rpm}$ and a flow rate of $300 \mathrm{~mL} / \mathrm{min}$. The test duration was 10 minutes, as before. Using the same disassembly and swabbing procedure, $6.4 \mathrm{mg}$ of solvent were collected from the heavy phase collector ring. Subsequently, testing was performed with an increased flow rate of $500 \mathrm{~mL} / \mathrm{min}$. This modification in test conditions resulted in diversion of $14.6 \mathrm{mg}$ of solvent into the heavy phase collector. After reassembly of the test apparatus, contactor rotation was resumed at an increased rate of $4200 \mathrm{rpm}$ and the solvent flow rate was returned to the previous value of $300 \mathrm{~mL} / \mathrm{min}$. Sampling after 10 minutes of operation resulted in collection of $6.3 \mathrm{mg}$ of solvent from the organic collector ring. Results of all testing, including replicates at each condition, are 
presented in Table 10.11. Minor differences between carryover values at the different speed and flow rate conditions fall within a narrow range, and are not significant enough to make a distinction between flow and rotation effects on carryover when the modified housing was used.

\subsection{EVALUATION OF CONTRACTOR AIR FLOWS}

The objective of this part of the test program was to determine the motivation for air flow through the contactor, as this impacts both the generation of mist and the gradual evaporation of volatile solvent components, the latter creating a constant process transient. As shown in Fig. 10.5, a column of air exists along the centerline of a centrifugal contactor during operation. Vanes located axially inside the rotor prevent slippage of liquid at the rotor wall, transferring angular acceleration from the rotor to the dispersion, effecting separation.

In ANL/ORNL/SRTC designs, these vanes are typically attached to the rotor wall and extend inward only as far as the light phase (usually organic) weir. In the CINC model V-2 the vanes extend to the centerline of the contactor. In CINC V-5 and V-10 models, the vanes extend to a central shaft that is used to facilitate internal flushing of the device. In all cases with the CINC units, the possibility exists of creating a slight vacuum in the region of the rotor's centerline due to outward acceleration of air away from the centerline, toward the liquid surface that extends from the rotor inlet to the light phase weir. In the case of the $\mathrm{V}-5$ and $\mathrm{V}-10$, this condition should be partially mitigated by the presence of the central shaft. In the case of ANL/ORNL/SRNL designs, creation of a slight vacuum by the action of the rotor vanes is essentially eliminated by the lack of vane surfaces in the region from the light phase weir to the centerline.

A second possible air motivator is the light phase discharge mechanism, i.e. the system of radial ports extending from the wall of the light phase collection chamber to the rotor's outer wall. Since there is no liquid seal within the rotor along the light phase pathway while the heavy phase pathway is sealed, any air present in the rotor must either remain there or exit with light phase effluent. At start up, air occupies the rotor, including the light phase discharge nozzles. It is reasonable to expect that this air is projected outward when rotor operation is started, creating a slight vacuum in the region of the light phase weir.

As it is possible that the flow of air accompanying light phase effluent could promote the generation of mist that could migrate into the heavy phase effluent path, testing was performed to isolate pressure/air flow effects within the rotor. 


\subsubsection{Procedure}

All testing was performed using a standard CINC Model V-2 contactor. Initial testing was performed using a rotor insert having internal vanes that extend across the diameter of the rotor, as received from the fabricator. This phase of testing was performed using a straight-vane bottom housing plate. A piece of $1 / 4^{-}$ in tubing was inserted through an opening on the bottom housing plate such that tubing extended into the bottom of the rotor but did not contact the vane insert. The tubing was locked in place and sealed to the housing using a bulkhead compression fitting. The other end of the tubing was submerged in a beaker filled with water. The rotor was started, slowly at first (in case there were any interferences), then the speed was increased to $2000 \mathrm{rpm}$. At this condition there was no indication of a pressure difference as no liquid was drawn into the tube. Flow (of water) was then initiated at $100 \mathrm{~mL} / \mathrm{min}$ and continued until aqueous effluent appeared at which point the feed pump was turned off. At this point during testing, a 1$2 \mathrm{~mm}$ lift of liquid into the pressure tube was noted. The rotor speed was then increased to $3600 \mathrm{rpm}$ and flow of liquid at $100 \mathrm{~mL}$ was resumed. As before, pumping was terminated when flow was observed from the aqueous effluent nozzle. At this condition a liquid level of $1 \mathrm{~cm} \mathrm{H}_{2} \mathrm{O}$ was observed in the pressure tube.

The contactor was disassembled and the vane insert was replaced with one having vanes extending only to the light phase weir. As before, the contactor speed was taken to $2000 \mathrm{rpm}$ without inlet flow. No pressure difference between the ambient atmosphere and the inside of the rotor was indicated. Again, a differential pressure of 1-2 mm was indicated at $2000 \mathrm{rpm}$ after operating the contactor with aqueous flow long enough to establish a liquid seal under the heavy phase underflows. As before, a pressure differential of $1 \mathrm{~cm} \mathrm{H}_{2} \mathrm{O}$ was observed after increasing the rotor speed and reestablishing flow long enough to ensure the presence of an aqueous seal inside the rotor.

Following these tests, the contactor was disassembled, the rotor was disconnected from the bearing housing, the vane insert was removed, and the contactor was reassembled. In addition, the bottom housing plate was replaced with the vane Less plate. This change was necessitated by the inability of the rotor to 'pump' liquids in the absence of internal vanes that would have resulted in flooding the region of the contactor where the upper end of the pressure tube was located.

Operation was initiated at a rotor speed of $2000 \mathrm{rpm}$ with no liquid present in the contactor. Under this condition there was no indication of pressure differential. At this point, $50 \mathrm{~mL}$ of water were introduced via one of the feed nozzles. With liquid present in the mixing zone at a rotor speed of $2000 \mathrm{rpm}$, no 
pressure differential was observed. Upon increasing the rotor speed to $3600 \mathrm{rpm}$, a pressure differential between ambient and the inside of the rotor of $2 \mathrm{~mm} \mathrm{H}_{2} \mathrm{O}$ was indicated.

The apparatus was drained and disassembled, and the partial diameter vane insert was reinstalled in the rotor. After reassembly, testing was repeated. With the internal vanes in place, there was no pressure differential in the liquid-free contactor at $2000 \mathrm{rpm},<1 \mathrm{~mm} \mathrm{H}_{2} \mathrm{O}$ differential after adding $50 \mathrm{~mL}$ liquid to the mixing zone, and 2-3 $\mathrm{mm}$ differential at $3600 \mathrm{rpm}$ with liquid in the mixing zone.

Finally, the rotor was disassembled, the full diameter vane insert was installed, and the contactor was reassembled for testing. With these vanes in place, there was $<1 \mathrm{~mm} \mathrm{H}_{2} \mathrm{O}$ pressure difference in the dry system at a rotor speed of $2000 \mathrm{rpm}$, the same pressure differential after adding $50 \mathrm{~mL} \mathrm{H}_{2} \mathrm{O}$ to the device, and a $3 \mathrm{~mm}$ differential at $3600 \mathrm{rpm}$ with liquid in the mixing zone. As configured in this test, the heavy phase flow path is unblocked. Consequently, any pressure reduction generated mechanically should be balanced by inflow of air across the heavy phase weir and through the underflows, into the rotor.

An additional test was performed with the rotor modified as in the entrainment tests; the heavy phase weir was blocked to prevent flow of air via this pathway when the rotor was operated without internal liquid. As in the previous test, the contactor was operated at $2000 \mathrm{rpm}$ and the height differential in a tube submerged in water was observed and recorded. The same observation was made after increasing the rotor speed to $3600 \mathrm{rpm}$. At the lower speed, a pressure differential of approximately $3 \mathrm{~mm}$ liquid was observed. Upon increasing the speed to $3600 \mathrm{rpm}$ the differential increased to $9-10 \mathrm{~mm}$, with regular pulsing between these values being observed.

\subsection{CONCLUSIONS}

\subsubsection{Property Determinations}

Results of dispersion number determinations indicate that all solution pairs considered for use in extraction, scrubbing, and stripping operations can be separated adequately in centrifugal contactors. Decreases in modifier (Cs-7SB) concentration were found to have a consistent effect of improving phase separation behavior across the process cascade, i.e., throughout extraction, scrubbing, and stripping. Dispersion numbers for stripping operations using $\mathrm{H}_{3} \mathrm{BO}_{3}$ at a concentration of $1 \mathrm{mM}$ are undesirably low; hence, it is recommended that a minimum acid concentration of $10 \mathrm{mM} \mathrm{H}_{3} \mathrm{BO}_{3}$ be used in stripping. As expected, dispersion number results are consistent with interfacial tension data, in that higher values of 
the later (indicating greater difficulty in interface breakage) were obtained for systems having higher dispersion numbers (indicating greater tendency for dispersion separation).

\subsubsection{Contactor Hydraulics}

Phase separation was good over a fairly wide range of conditions, but not at throughputs approaching the vendor-claimed processing capacity of $>2 \mathrm{~L} / \mathrm{min}$. Under extraction conditions performance was acceptable for the $50 \mathrm{mM}$ MaxCalix solvent formulation at the phase ratios considered for use in the MCU, but, at flows less than $50 \%$ the manufacturer-stated maximum. Acceptable separation under scrubbing and stripping conditions was limited to throughputs below $600 \mathrm{~mL} / \mathrm{min}$ total flow. It is important to note the aqueous samples were found to be free of visible organic contamination in all cases, and that increases in organic contamination of aqueous effluents were observed as a function of time (with all other factors being constant). The latter indication is believed to be indicative of an approach to steady-state entrainment resulting from factors external to the rotor.

\subsubsection{Mass Transfer}

Stage efficiencies obtained under both extraction and stripping conditions were adequate for achieving MCU DF targets using the existing MCU stage configuration. The results under the extraction condition were consistently greater than $90 \%$. Stripping results using a fully pumping rotor were low, but were consistent with results obtained during similar testing of the BOBCalixC6 CSSX chemistry that was performed in 2001. As in the earlier tests, improved mass transfer efficiency was obtained by increasing residence time in the mixing zone of the contactor, which was accomplished through use of a partially pumping rotor.

Given that stripping results in the most recent test are comparable to those obtained in prior BOBCalixC6 CSSX testing and that BOBCalixC6 CSSX processing has been performed successfully in MCU, it is concluded that NG-CSSX operating in the existing MCU flowsheet will also be successful.

\subsubsection{Air Flow and Entrainment Evaluations}

Testing performed using a variety of internal vanes - full diameter, partial diameter, and no vanesindicate that generation of a slight negative pressure in the air space located along the centerline of the rotor is not due to the internal rotor vanes. Furthermore, the source of the pressure effect appears to be the configuration of the light phase discharge ports and the upper end of the rotor, as the effect was present when no vanes were in place. The pressure effect includes a slight, regular and periodic fluctuation that is 
consistent with liquid pulsing in the mixing zone of the contactor. The combined observations explain the evaporation of solvent components during operation, which results in a minor transient condition that requires supplemental addition of solvent. Elimination of the pressure effect and the flow it induces would require a design modification of some significance.

The transfer of 'less dense' organic phase material into the heavy phase collector ring was indicated during testing. Corresponding transfer of heavy phase material (aqueous solution) in the opposing collector ring was not. The difference is likely a reflection that the light phase discharge is a discharge of both liquid and gas and that the organic material used has a lower surface tension than the aqueous solution, than the result of a difference in collector ring configuration. Increasing throughput tended to exacerbate the problem, while an increase in rotor speed had minimal effect, if any. This observation is consistent with the mitigation of entrainment issues in MCU by reduction of processing throughput.

A modification to the collector ring did result in a consistent and significant reduction in the quantity of organic solution transferred into the aqueous flow path. At a minimum (within the data scatter), a 15-fold reduction in carryover is indicated. 


\section{EFFECT OF POSSIBLE IMPURITIES ON PERFORMANCE}

This work was undertaken to provide a better understanding of the effects that impurities and solventbreakdown products could have on the NG-CSSX process. A question regarding the NG-CSSX process relative to the baseline BOBCalixC6 CSSX process now employed in the MCU is what effect the decomposition products of the new suppressor (i.e., $N, N^{\prime}$-dicyclohexyl- $N^{\prime \prime}$-isotridecylguanidine) used in the NG-CSSX process could have on cesium distribution in extraction, scrubbing, and stripping. It was found that a set of anticipated guanidine breakdown products at $1 \mathrm{mM}$ initial concentration in the NGCSSX solvent (i.e., with an assumed decomposition of a third of the guanidine with no mitigation) has a negligible effect on ESS performance. It was also shown that the known 4-sec-butylphenol (SBP) decomposition product of the Cs-7SB modifier at a concentration of $10 \mathrm{mM}$ in the NG-CSSX solvent impairs stripping performance, approximately to the same degree as already known for BOBCalixC6 CSSX. However, the SBP is readily washed from the solvent, and its buildup to such a level is not expected in normal operation. Initial results thus indicate no particular sensitivity to impurities that have not been previously encountered in BOBCalixC6 CSSX. However, the actual NG-CSSX degradation products have not yet been measured, and future work is recommended to analyze degraded solvent and to determine the fate and effect of degradation products.

\subsection{INTRODUCTION}

Under funding from the DOE-EM Office of Technology Innovation and Development, recent laboratory efforts have been directed toward developing the NG-CSSX process for cesium removal from alkaline tank wastes ${ }^{1}$. In support of EM's drive to accelerate processing of tank wastes at the SRS and Hanford sites $^{2}$, laboratory efforts have sought increased performance over the current baseline, both in decontamination efficiency and waste-processing rate ${ }^{3}$. The ability of the NG-CSSX solvent to properly function in the presence of impurities resulting from either the gradual degradation of solvent components or impurities present in tank waste is a major question pertaining to the long-term stability of process performance. Toward an initial answer to this question, the sensitivity of NG-CSSX performance to anticipated breakdown products was assessed by monitoring the $D_{\mathrm{Cs}}$ in batch ESS tests.

The NG-CSSX solvent components and tested impurities are shown in Table 11.1. A description of the function of the NG-CSSX solvent components has been provided previously ${ }^{3}$. The primary sources of the impurities of most interest, at least at this stage of our understanding of the chemistry of NG-CSSX, are the degradation products of the guanidine suppressor, $N, N^{\prime}$-dicyclohexyl- $N$ "-isotridecylguanidine (DCiTG) and the modifier, 1-(2,2,3,3-tetrafluoropropoxy),-3-[4-(sec-butyl)phenoxy]-2-propanol (Cs- 
7SB). The guanidine introduces new chemistry not present in BOBCalixC6 CSSX, which uses tri-noctylamine (TOA) as the suppressor. Although the actual DCiTG breakdown products have not been identified, an educated projection of possible species can be made. They include $N, N^{\prime}$-dicyclohexylurea (DCU), iTDA, and $N, N^{\prime}, N^{\prime}$-tricyclohexylguanidine (TCHG), which have already been encountered as impurities in the synthesis of DCiTG (see Chapter 4) and may therefore be present at low levels even in pristine solvent. In the case of Cs-7SB, it has already been shown that the major decomposition product of concern, both from chemical-thermal and radiolytic pathways, is $\mathrm{SBP}^{7}$, also a minor impurity in pristine solvent. Potential degradation products of MaxCalix were not evaluated at this time, being regarded as relatively low risk, given the structural similarity of MaxCalix to the calixarene BOBCalixC6 used in BOBCalixC6 CSSX, which has been found to be robust both in degradation tests in the prior BOBCalixC6 CSSX development ${ }^{7}$ and in actual MCU performance.

Table 11.1. NG-CSSX solvent components and possible degradation products

\begin{tabular}{|c|c|c|c|}
\hline Component & Code & Chemical Name & Structure \\
\hline Extractant & MaxCalix & $\begin{array}{l}\text { 1,3- alt-25,27-Bis }(3,7- \\
\text { dimethyloctyl-1-oxy) } \\
\text { calix[4]arene-benzocrown- } \\
6\end{array}$ & \\
\hline Modifier & Cs-7SB & $\begin{array}{l}\text { 1-(2,2,3,3- } \\
\text { Tetrafluoropropoxy)-3-(4- } \\
\text { sec-butylphenoxy)-2- } \\
\text { propanol }\end{array}$ & $\mathrm{F}_{2} \mathrm{CF}_{2} \mathrm{H}$ \\
\hline Suppressor & $\begin{array}{l}\text { DCiTG or } \\
\text { LIX } 79\end{array}$ & $\begin{array}{l}N, N^{\prime} \text {-Dicyclohexyl- } N " \text { - } \\
\text { isotridecylguanidine }\end{array}$ & \\
\hline Diluent & Isopar L & $\begin{array}{l}\mathrm{C}_{12} \text {-isoparaffinic } \\
\text { hydrocarbon }\end{array}$ & \\
\hline $\begin{array}{l}\text { DCiTGnidine } \\
\text { Impurity } 1\end{array}$ & DCU & $N, N^{\prime}$-Dicyclohexylurea & \\
\hline $\begin{array}{l}\text { DCiTGnidine } \\
\text { Impurity } 2\end{array}$ & iTDA & Isotridecylamine & $\begin{array}{c}i \mathrm{C}_{13} \mathrm{H}_{27} \mathrm{NH}_{2} \text { (branched alkyl chain averaging } \\
\text { approx. } 10 \text { carbons long) }\end{array}$ \\
\hline $\begin{array}{l}\text { DCiTGnidine } \\
\text { Impurity } 3\end{array}$ & TCHG & $\begin{array}{l}N, N, N N^{\prime}- \\
\text { Tricyclohexylguanidine }\end{array}$ & \\
\hline Modifier Impurity & SBP & 4-sec-Butylphenol & \\
\hline
\end{tabular}


The present results, together with the results from the thermal and radiolytic stability testing (Chapter 12), should serve as an indication of the sensitivity of solvent performance to specific potential impurities and related species.

The second major source of impurities is the tank waste being treated, which is already known to contain a certain level of the alky-phosphates, mono-, di-, and tri-butyl phosphate. Hence, a second group of possible impurities are surfactants, long chain lipophilic anionic impurities, which could potentially enter the flowsheet by a number of means. SDS was chosen as a surfactant that could be traced and two further systems were tested containing different amounts of SDS (see Table 11.2). In order to test how these impurities behave in a bench top process, simulants of various compositions were subjected to cold $\mathrm{ES}_{2} \mathrm{~S}_{3}$ testing and the aqueous feeds were subsequently analyzed for phosphorous and sulfur using ICP-AES.

Table 11.2. Partial target compositions of aqueous tank-waste simulants used for ICP-AES analysis*

\begin{tabular}{|c|c|c|c|c|c|}
\hline Analyte or Species & $\begin{array}{l}\text { SRS-15 } \\
\mathrm{mol} / \mathrm{L}\end{array}$ & $\begin{array}{l}\text { SRS-45 } \\
\mathrm{mol} / \mathrm{L}\end{array}$ & $\begin{array}{c}\text { Hanford } \\
\mathrm{mol} / \mathrm{L}\end{array}$ & $\begin{array}{c}\mathrm{SDS} \\
\mathrm{mol} / \mathrm{L}\end{array}$ & $\begin{array}{c}\text { SDS-spike } \\
\mathrm{mol} / \mathrm{L}\end{array}$ \\
\hline \multicolumn{6}{|l|}{ Principal constituents: } \\
\hline $\mathrm{Al}$ as $\left(\mathrm{Al}(\mathrm{OH})_{4}{ }^{-}\right)$ & 0.280 & 0.280 & 0.570 & 0.280 & 0.280 \\
\hline $\mathrm{Cl}^{-}$ & $2.4 \times 10^{-2}$ & $2.4 \times 10^{-2}$ & $9.4 \times 10^{-2}$ & $2.4 \times 10^{-2}$ & $2.4 \times 10^{-2}$ \\
\hline $\mathrm{NO}_{2}^{-}$ & 0.500 & 0.500 & 1.17 & 0.500 & 0.500 \\
\hline $\mathrm{NO}_{3}^{-}$ & 2.03 & 2.06 & 1.90 & 2.03 & 2.03 \\
\hline $\mathrm{OH}^{-}$(Free) & 2.06 & 2.06 & 1.45 & 2.06 & 2.06 \\
\hline $\mathrm{SO}_{4}{ }^{2-}$ & 0.140 & 0.140 & $3.22 \times 10^{-2}$ & 0.140 & \\
\hline \multicolumn{6}{|l|}{ Minor organic constituents: } \\
\hline Tri-n-butylphosphate (TBP) & $1.9 \times 10^{-6}$ & $1.9 \times 10^{-6}$ & & $1.9 \times 10^{-6}$ & \\
\hline Di-n-butylphosphate (DBP) & $1.2 \times 10^{-4}$ & $1.2 \times 10^{-4}$ & & $1.2 \times 10^{-4}$ & \\
\hline Mono-n-butylphosphate (MBP) & $1.6 \times 10^{-4}$ & $1.6 \times 10^{-4}$ & & $1.6 \times 10^{-4}$ & \\
\hline Formate $\left(\mathrm{HCO}_{2}^{-}\right)$ & $3.3 \times 10^{-2}$ & $3.3 \times 10^{-2}$ & & $3.3 \times 10^{-2}$ & \\
\hline Oxalate $\left(\mathrm{C}_{2} \mathrm{O}_{4}{ }^{2-}\right)$ & $8.0 \times 10^{-3}$ & $8.0 \times 10^{-3}$ & $1.0 \times 10^{-2}$ & $8.0 \times 10^{-3}$ & \\
\hline Trimethylamine & $1.7 \times 10^{-4}$ & $1.7 \times 10^{-4}$ & & $1.7 \times 10^{-4}$ & \\
\hline Sodium dodecyl sulfate (SDS) & & & & $1.0 \times 10^{-3}$ & $1.0 \times 10^{-3 * *}$ \\
\hline
\end{tabular}

*For full aqueous tank-waste simulant compositions see Chapter 2.

**Component spiked into simulant whilst in contact with the NG-CSSX solvent. Components of interest for this study highlighted in bold.

\subsection{EXPERIMENTAL SECTION}

\subsubsection{Materials}

Solvent components were obtained from commercial sources and judged to be of adequate purity for use as received. DCiTG was synthesized as an $\mathrm{HCl}$ salt. 1-(2,2,3,3-Tetrafluoropropoxy),-3-[4-(secbutyl)phenoxy]-2-propanol (Cs-7SB modifier, Lot No. MOD2010-M-2) and 1,3-alt-25,27-bis(3,7- 
dimethyloctyl-1-oxy)calix[4]arene-benzocrown-6 (MaxCalix, Lot No. 71-061-15) were obtained from Marshallton Research Laboratories, and Isopar L (Lot No. US67377A) was obtained from ExxonMobil. Solvents were prepared by weighing appropriate amounts of extractant, modifier, and suppressor (DCiTG) into volumetric flasks and diluting with Isopar L to the mark. The iTDA and $N, N^{\prime}, N$ ',tricyclohexylguanidine were synthesized according to reported procedures (Chapter 4). N,N'Dicyclohexylurea and the cyclohexylamine starting material for the $N, N$ ', $N$ "-tricyclohexylguanidine were obtained from Aldrich. 4-sec-Butylphenol was obtained from TCI America. All chemicals obtained from commercial sources were used as received.

Multiple solvents comprised of 0.05 M MaxCalix, 0.5 M Cs-7SB modifier, and either 0.003 or $0.002 \mathrm{M}$ DCiTG guanidine in Isopar L used in the contacting experiments were prepared and prewashed. The prewashes were carried out in following manner: one contact sequentially with $0.010 \mathrm{M} \mathrm{HCl}, \mathrm{H}_{2} \mathrm{O}$, then decreasing concentrations of $\mathrm{NaOH}(0.3 \mathrm{M}, 0.1 \mathrm{M}, 0.03 \mathrm{M}$, and $0.01 \mathrm{M})$, and then once or twice with $\mathrm{H}_{2} \mathrm{O}$ until the solution was $\mathrm{pH}$ neutral. The components used to simulate the possible impurities/breakdown products were added to the solvents after the solvents were washed. This was done to prevent the washing-out of simulated decomposition products.

The aqueous phase used in the ESS tests was the SRS tank-waste simulant SRS-15, whose composition and preparation has been described in detail previously ${ }^{3,7,9}$.

Multiple solvents comprised of 0.05 M MaxCalix, 0.5 M Cs-7SB modifier, and either 0.003 or $0.002 \mathrm{M}$ DCiTG guanidine in Isopar L used in the contacting experiments were prepared and prewashed. The prewashes were carried out in following manner: one contact sequentially with $0.010 \mathrm{M} \mathrm{HCl}, \mathrm{H}_{2} \mathrm{O}$, then decreasing concentrations of $\mathrm{NaOH}(0.3 \mathrm{M}, 0.1 \mathrm{M}, 0.03 \mathrm{M}$, and $0.01 \mathrm{M})$, and then once or twice with $\mathrm{H}_{2} \mathrm{O}$ until the solution was $\mathrm{pH}$ neutral. The components used to simulate the possible impurities/breakdown products were added to the solvents after the solvents were washed. This was done to prevent the washing-out of simulated decomposition products.

\subsubsection{Methods}

\subsubsection{Cesium distribution ratios $\left(D_{\mathrm{Cs}}\right)$}

$D_{\mathrm{Cs}}$ with simulants were obtained in duplicate in a manner similar to that described previously ${ }^{3}$ with $\mathrm{ES}_{2} \mathrm{~S}_{3}$. Phases were contacted in polypropylene micro-tubes mounted by clips on a disk rotated for 60 minutes for extractions and 45 minutes for scrubs and strips. The solutions were contacted inside of an 
air-box maintained at a temperature of $25 \pm 0.2{ }^{\circ} \mathrm{C}$. After the contacting period, the tubes were centrifuged for 5 minutes at 3000 RPM at the experimental temperature. An appropriate aliquot of each phase was subsampled and counted using a Packard Cobra II Auto-Gamma counter. A spike of Cs-137 was added to the second and third aqueous strip solutions, owing to the low number of counts remaining after the each strip. To keep samples at the equilibration temperature, tubes were removed individually from the temperature-controlled centrifuge for subsampling. Based on the agreement between duplicate samples, the precision of $D_{\mathrm{Cs}}$ values is $\pm 5 \%$ (extraction and scrub), $\pm 10 \%$ (first strip), and $\pm 30 \%$ (second and third strips). $D_{\mathrm{Cs}}$ are given as the ratio of the background-corrected volumetric count rates of the radioisotope in each phase at equilibrium.

\subsubsection{Aqueous feed sulfur and phosphorus concentration}

The solvent used in these experiments comprised of MaxCalix (0.05 M), Cs-7SB modifier $(0.5 \mathrm{M})$, and DCiTG L guanidine suppressor $(0.003 \mathrm{M})$ in Isopar L. The solvent was prepared and prewashed prior to use in the following manner: sequential contacts (single contact with an $\mathrm{O}: \mathrm{A}$ of $1: 1$ ) with $0.010 \mathrm{M} \mathrm{HCl}$, $\mathrm{H}_{2} \mathrm{O}$, then decreasing concentrations of $\mathrm{NaOH}(0.3 \mathrm{M}, 0.1 \mathrm{M}, 0.03 \mathrm{M}$, and $0.01 \mathrm{M})$, and then with $\mathrm{H}_{2} \mathrm{O}$ until the solution was $\mathrm{pH}$ neutral.

ESS analysis using each of the waste simulants was carried out in a manner similar to that described in Chapter 2, after the contacting period, the tubes were centrifuged for 5 minutes at 3000 RPM at the experimental temperature. The aqueous and organic phases from the scrub and strip stages were separated and the aqueous phases were subsampled $(1.0 \mathrm{~mL})$ and diluted ten-fold with deionized water for ICP analysis.

An IRIS Intrepid II XSP Inductively Coupled Plasma Atomic Emission spectrometer was used for sample analysis using the Thermo elemental TEVA ${ }^{\circledR} 2001$ software. The machine was calibrated using standards purchased from High Purity Standards.

Table 11.3. Wavelengths used for ICP-AES analysis given along with the correlation of the calibration curve for each analyte and the precision of the measurements $<10 \mathrm{ppm}$

\begin{tabular}{lccc}
\hline Element & Wavelength $(\mathbf{n m})$ & Calibration $\mathbf{R}^{\mathbf{2}}$ & Observed precision at $<\mathbf{1 0} \mathbf{~ p p m}$ \\
\hline $\mathrm{Na}$ & 589.592 & 0.999 & $\pm 0.50 \mathrm{ppm}$ \\
$\mathrm{K}$ & 766.491 & 0.999 & $\pm 0.10 \mathrm{ppm}$ \\
$\mathrm{B}$ & 249.773 & 0.999 & $\pm 0.25 \mathrm{ppm}$ \\
$\mathrm{S}$ & 180.731 & 0.999 & $\pm 0.02 \mathrm{ppm}$ \\
$\mathrm{P}$ & 177.499 & 0.999 & $\pm 0.02 \mathrm{ppm}$ \\
$\mathrm{Al}$ & 308.215 & 0.999 & $\pm 0.25 \mathrm{ppm}$ \\
\hline
\end{tabular}


Mixed element standards comprised of phosphorus and sulfur 1, 2.5, 5, 7.5, 10, 25, 50, 100 and 500ppm were prepared $(50 \mathrm{~mL}$ in deionized water). The wavelengths used for each element are given in Table 11.3 along with the correlation, $\mathrm{R}^{2}$, of the calibration curve obtained. Each experiment was carried out in duplicate and the analysis of each sample was carried out in quadruplicate.

\subsection{RESULTS AND DISCUSSION}

\subsubsection{Potential Breakdown Product Impurity Testing}

The compositions of the solvents tested are given in Table 11.4. The concentrations of the impurities were chosen as a possible worst case where $1 / 3$ of the guanidine has decomposed with breakdown products remaining in the solvent (instead of being washed out). This is overly conservative because iTDA and TCHA are less lipophilic than DCiTG and are expected to be washed out of the solvent primarily in the strip effluent. The tested concentration of the decomposition product of the modifier, SBP, was greatly exaggerated at $2 \%$ loss or $0.010 \mathrm{M}$, which was earlier seen to be the approximate concentration corresponding to onset of stripping failure in BOBCalixC6 CSSX. The controls included the baseline NGCSSX solvent $^{3}$ and the analogous solvent with only $2 / 3$ of the baseline DCiTG concentration.

No effect of the presence of possible DCiTG decomposition products on ESS performance was detectable at the level of $1 \mathrm{mM}$ in the solvent. Table 11.5 presents the values determined for $D_{C s}$ in standard $\mathrm{ES}_{2} \mathrm{~S}_{3}$ batch tests of the various NG-CSSX solvents containing impurities. The $0.002 \mathrm{M}$ control represents the effect of $1 / 3$ loss of DCiTG but with no impurities. It may be seen that the $1 / 3$ loss of DCiTG has no effect on extraction or scrubbing, but stripping is slightly worsened. In comparison, the DCU, iTDA, DCU + iTDA, and TCHA solvents also exhibited no change in extraction and scrubbing. Among these solvents, only the TCHA exhibited a change in stripping, which was a minor worsening of no significance.

Table 11.4. Solvent compositions used in ESS experiments ${ }^{a}$

\begin{tabular}{ccccclll}
\hline Solvents & $\begin{array}{c}\mathbf{0 . 0 0 3} \mathbf{M} \\
\text { Control }\end{array}$ & $\begin{array}{c}\mathbf{0 . 0 0 2} \mathbf{M} \\
\text { Control }\end{array}$ & SBP & DCU & iTDA & $\begin{array}{c}\text { DCU }+ \\
\text { iTDA }\end{array}$ & TCHA \\
\hline MaxCalix & $0.05 \mathrm{M}$ & $0.05 \mathrm{M}$ & $0.05 \mathrm{M}$ & $0.05 \mathrm{M}$ & $0.05 \mathrm{M}$ & $0.05 \mathrm{M}$ & $0.05 \mathrm{M}$ \\
Cs-7SB & $0.5 \mathrm{M}$ & $0.5 \mathrm{M}$ & $0.5 \mathrm{M}$ & $0.5 \mathrm{M}$ & $0.5 \mathrm{M}$ & $0.5 \mathrm{M}$ & $0.5 \mathrm{M}$ \\
DCiTG & $0.003 \mathrm{M}$ & $0.002 \mathrm{M}$ & $0.003 \mathrm{M}$ & $0.002 \mathrm{M}$ & $0.002 \mathrm{M}$ & $0.002 \mathrm{M}$ & $0.002 \mathrm{M}$ \\
Impurities & None & None & SBP & DCU & iTDA & DCU and & TCHG \\
& & & $0.01 \mathrm{M}$ & $0.001 \mathrm{M}$ & $0.001 \mathrm{M}$ & iTDA, each & $0.001 \mathrm{M}$ \\
& & & & & & at $0.001 \mathrm{M}$ & \\
\hline
\end{tabular}

\footnotetext{
${ }^{a}$ The components are dissolved in Isopar L diluent, an isoparafinic hydrocarbon.
} 
Anticipated impact of DCU, iTDA, and TCHA on process performance is expected to be negligible. As bases, iTDA and TCHA are more hydrophilic than the larger DCiTG and are expected to transfer to the acidic strip effluent stream. Work in progress, which will be presented in a subsequent report, shows that the synthesized DCiTG material has a partition ratio on the order of 50 in contact with the strip solution. Given that TCHA, iTDA, and any guanidine derivative of DCiTG formed by loss of an alkyl group would have at least 6 aliphatic carbons less than DCiTG and therefore would partition $\geq 1000$-fold more strongly to the boric acid strip solution than DCiTG, no buildup of any such decomposition product would be expected. Assuming that up to $1 \mathrm{mM}$ of these basic impurities could form under any unusual conditions of solvent use or handling, no effect of TCHA or iTDA would be expected based upon the ESS test results in Table 3. On the other hand, DCU is more lipophilic, with an estimated ClogP of 3.5 (CS ChemDraw Ultra 12.0), meaning that the estimated partition ratio of DCU between 1-octanol and water is 3200. If its rate of production is conservatively assumed to be $1 \mathrm{mM}$ per 1000 solvent cycles (unknown but likely conservative), then a loss solely through stripping would correspond to a steady-state buildup in the solvent to give $12 \mathrm{mM}$ DCU after thousands of solvent cycles. This is a larger concentration than tested in this report, but given that the effect of this neutral oxygen-donor compound would be similar to that of tri-n-butylphosphate, found to have no effect on ESS up to $30 \mathrm{mM}$ in BOBCalixC6 CSSX solvent ${ }^{7}$, no effect of DCU is anticipated, despite its low loss to the aqueous effluent phases.

Table 11.5. $D_{\mathrm{Cs}}$ for ESS batch tests ${ }^{\mathrm{a}}$

\begin{tabular}{|c|c|c|c|c|c|c|c|}
\hline Solvent & $\begin{array}{l}\text { 0.003 M } \\
\text { Control }\end{array}$ & 0.002 M Control & SBP & DCU & iTDA & $\begin{array}{l}\text { DCU + } \\
\text { iTDA }\end{array}$ & ТСНА \\
\hline Extraction & $52(1)$ & $54.1(6)$ & $74(1)$ & $59(3)$ & $55(2)$ & $53.4(8)$ & $53.9(3)$ \\
\hline Scrub 1 & $2.20(0)$ & $2.30(0)$ & $29.3(7)$ & $2.30(5)$ & $3(1)$ & $2.4(1)$ & $2.6(1)$ \\
\hline Scrub 2 & $0.90(1)$ & $0.90(1)$ & $26.4(3)$ & $0.90(2)$ & $0.90(5)$ & $0.5(6)$ & $0.90(6)$ \\
\hline Strip 1 & $1.2(2) \times 10^{-3}$ & $1.3(1) \times 10^{-3}$ & $1.6(2) \times 10^{-1}$ & $1.4(3) \times 10^{-3}$ & $1.4(1) \times 10^{-3}$ & $1.23(2) \times 10^{-3}$ & $2.05(8) \times 10^{-3}$ \\
\hline Strip 2 & $2(1) \times 10^{-4}$ & $4.1(3) \times 10^{-4}$ & $5.5(3) \times 10^{-3}$ & $1.0(7) \times 10^{-3}$ & $3.86(4) \times 10^{-4}$ & $4.2(5) \times 10^{-4}$ & $6.1(2) \times 10^{-4}$ \\
\hline Strip 3 & $4(1) \times 10^{-4}$ & $3.53(9) \times 10^{-4}$ & $8(2) \times 10^{-4}$ & $2.77(6) \times 10^{-4}$ & $3(2) \times 10^{-4}$ & $6(3) \times 10^{-4}$ & $3(1) \times 10^{-4}$ \\
\hline
\end{tabular}

${ }^{a}$ The aqueous phases were SRS- 15 waste simulant for extraction, $0.025 \mathrm{M} \mathrm{NaOH}$ for scrubs, and $0.01 \mathrm{M} \mathrm{H}_{3} \mathrm{BO}_{3}$ for strips. Solvent compositions are defined in Table 2. The O:A ratios were 1:4 for extraction and 3.75:1 for both scrubbing and stripping. The temperature was $25.0 \pm 0.2^{\circ} \mathrm{C}$.

The only significant change in the $D_{C s}$ values in the ESS batch testing appeared when $10 \mathrm{mM}$ SBP is present as the impurity. This concentration is much larger than would be credible, but it represents the level for onset of stripping failure in the BOBCalixC6 CSSX process ${ }^{7}$. As may be seen from Table 11.5, the addition of $10 \mathrm{mM} \mathrm{SBP}$ to the solvent enhances the $D_{C s}$ values in all steps. Its effect on extraction and scrubbing is certainly due to its conversion to the anionic 4-sec-butylphenolate form, which would synergistically enhance cesium extraction. A way to visualize this is the strongly favored exchange of the organic-phase $\mathrm{Na}^{+}$cations, associated with the $\mathrm{SBP}^{-}$anions and weakly bound by MaxCalix, for aqueous- 
phase $\mathrm{Cs}^{+}$ions, extracted and strongly bound by MaxCalix. This behavior would hold under the alkaline conditions of extraction and scrubbing, but on stripping, the SBP would be expected to be reprotonated by the boric acid. As in BOBCalixC6 CSSX, stripping conditions can therefore tolerate fairly high concentrations of SBP, no longer in the anion form. The effect therefore must arise in the capacity of the organic-phase $\mathrm{SBP}^{-}$anions to consume protons from the aqueous acid solutions. In BOBCalixC6 CSSX, this reaction affected the $50 \mathrm{mM}$ and $1 \mathrm{mM} \mathrm{HNO}_{3}$ scrub and strip solutions, respectively, where at $\mathrm{O}: \mathrm{A}=$ 5 the system would fail at $10 \mathrm{mM} \mathrm{SBP}$, sufficient to neutralize all of the scrub acid. In the NG-CSSX system, impaired stripping is expected when the boric acid strip acid is depleted by reaction with the SBP anions. Given that stripping $D_{\mathrm{Cs}}$ values increase with increasing $\mathrm{pH}$ as the boric acid is neutralized ${ }^{11}$, an increasing concentration of $\mathrm{SBP}^{-}$in the solvent is expected to induce higher $D_{\mathrm{Cs}}$ strip values. In effect, this is what is seen in Table 11.5. The first strip $D_{\mathrm{Cs}}$ value is high, and with successive contacts with fresh $10 \mathrm{mM}$ boric acid, the $D_{\mathrm{Cs}}$ values drop to near normal by the third strip contact. Thus, the observed effect of SBP if allowed to build up in the solvent is consistent with neutralization of the boric acid strip solution, rendering it less effective in stripping the cesium.

\subsubsection{ICP Analysis for SDS and Phosphate Containing Impurities}

The organic phosphate and sulfate impurities found in the majority of the simulants tested are shown to be extracted to a certain extent although appear to be almost completely removed in the scrubbing stages.

\subsubsection{Effect of simulant on sulfur concentration}

The major sulfur containing species in the simulants are sulfate and, where added, dodecyl sulfate (Table 11.2). For the SRS-15, SRS-45 and Hanford simulants no measurable sulfur was observed and only low levels of sulfur were transferred for the SDS and cesium-free simulants upon scrubbing. The SDS-spiked simulant did, however, show the transfer of more significant amounts of sulfur and, as this simulant was sulfur free apart from the SDS, this tends to suggest that no sulfate is normally transferred to the solvent upon extraction. The major difference between the two SDS-containing simulants was the stage at which the SDS was added. For the SDS simulant, the SDS was added to the simulant together with the remaining components and the simulant was then allowed to settle. Due to the reduced solubility of the SDS in the highly ionic solvent, it is possible that the concentration of SDS is not accurate due to precipitation. For the SDS-spiked simulant the SDS was added only upon contact with the solvent, resulting in a much more accurate SDS concentration upon extraction. Only the SDS-spiked simulant gave any recordable sulfur transfer to the aqueous phase upon stripping. The sulfur transferal increases on 
consecutive strips, arising from neutralization of the boric acid; and, hence, removial of the alkaline metals before washing out the sulfur containing species.

\subsubsection{Effect of simulant on phosphorus concentration}

The total phosphorus transferred from the solvent upon scrubbing is shown in Table 11.6. The SRS-based solvents show total levels of phosphorus transferred of between 0.05 and $0.09 \mathrm{mM}$. It can be assumed that the phosphorus transferred for the SRS-based simulants is from the organic phosphate impurities as both the Hanford simulant and the SDS-spike simulant contain $0.134 \mathrm{mM}$ and $0.07 \mathrm{mM}$ of inorganic phosphate respectively, yet show very little transferred phosphorus.

If all of the organic phosphates in the simulant, about $0.28 \mathrm{mM}$, were extracted the total NG-CSSX solvent concentration would be $1.2 \mathrm{mM}$. If only the polyalkyl phosphates were extracted the concentration in the solvent would be $0.48 \mathrm{mM}$. The partitioning $\left(\log \mathrm{P}_{\text {Octano/ Water }}\right)$ of mono- and dibutyl phosphate between octanol and water has been determined experimentally as 2.017 and 0.179 respectively ${ }^{35}$ which correspond well with the calculated values of 2.38 and 0.68 (calculated using the chemaxon.marvin.plugin.calculator program ${ }^{36}$ at www.chemicalize.org). Were these values to be applied in the NG-CSSX extraction stage one could calculate a total organic concentration of around $0.92 \mathrm{mM}$. It has been reported ${ }^{35}$ that in basic media mono-n-butylphosphate and dibutylphenol will exist almost exclusively in the salt form resulting in considerably lower partitioning into an organic phase. The high ionic strength of the simulants may, however, salt out the alkyl phosphate salts into the solvent to a greater extent. For the SRS-based simulants, if the polyalkyl phosphates remain in the NG-CSSX solvent after extraction, the percentage of removed on scrubbing has been determined to be between 43 and $76 \%$.

These data clearly pertain to both the importance and efficacy of the $\mathrm{NaOH}$ scrubbing stage in removing impurities from the solvent in the NG-CSSX process, which in turn enables the boric acid stripping chemistry to remain effective. 


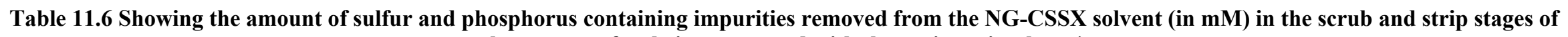
the process after being contacted with the various simulants*

\begin{tabular}{|c|c|c|c|c|c|c|c|c|c|c|c|c|}
\hline \multirow{2}{*}{$\begin{array}{l}\text { Simulant } \\
\text { Element }\end{array}$} & \multicolumn{2}{|c|}{ SRS-15 } & \multicolumn{2}{|c|}{ SRS-45 } & \multicolumn{2}{|c|}{ Hanford } & \multicolumn{2}{|c|}{ Cs-Free } & \multicolumn{2}{|r|}{ SDS } & \multicolumn{2}{|c|}{$\begin{array}{c}\text { S, K, TM, Org } \\
\text { Free - SDS Spike*** }\end{array}$} \\
\hline & $\mathrm{S}$ & $\mathrm{P}$ & $\mathrm{S}$ & $\mathrm{P}$ & $\mathrm{S}$ & $\mathrm{P}$ & $\mathrm{S}$ & $\mathrm{P}$ & $\mathrm{S}$ & $\mathrm{P}$ & $\mathrm{S}$ & $\mathrm{P}$ \\
\hline Scrub 1 & $0.003(1)$ & $0.185(2)$ & $0.002(2)$ & $0.124(2)$ & $0.001(1)$ & $0.011(2)$ & $0.010(1)$ & $0.247(2)$ & $0.007(1)$ & $0.141(3)$ & $0.058(1)$ & $<0.01$ \\
\hline Scrub 2 & $0.003(1)$ & $0.085(2)$ & $<0.001$ & $0.081(2)$ & $<0.001$ & $<0.01$ & $0.018(1)$ & $0.113(2)$ & $<0.001$ & $0.072(2)$ & $0.0195(7)$ & $<0.01$ \\
\hline $\begin{array}{l}\text { Scrub } \\
\text { total }\end{array}$ & $0.006(2)$ & $0.270(4)$ & $0.002(2)$ & $0.201(4)$ & $0.001(1)$ & $0.015(5)$ & $0.028(2)$ & $0.361(3)$ & $0.007(1)$ & $0.214(5)$ & $0.077(2)$ & $<0.01$ \\
\hline Strip 1 & $0.002(1)$ & $<0.01$ & $<0.001$ & $<0.01$ & $<0.001$ & $<0.01$ & $<0.001$ & $<0.01$ & $0.001(1)$ & $<0.01$ & $0.001(1)$ & $<0.01$ \\
\hline Strip 2 & $<0.001$ & $<0.01$ & $<0.001$ & $<0.01$ & $<0.001$ & $<0.01$ & $<0.001$ & $<0.01$ & $0.001(1)$ & $<0.01$ & $0.003(1)$ & $<0.01$ \\
\hline Strip 3 & $<0.001$ & $<0.01$ & $<0.001$ & $<0.01$ & $<0.001$ & $<0.01$ & $<0.001$ & $<0.01$ & $<0.001$ & $<0.01$ & $0.015(1)$ & $<0.01$ \\
\hline Strip total & $0.002(1)$ & $<0.01$ & $<0.001$ & $<0.01$ & $<0.001$ & $<0.01$ & $<0.001$ & $<0.01$ & $0.002(2)$ & $<0.01$ & $0.019(3)$ & $<0.01$ \\
\hline
\end{tabular}

* "Concentration removed from solvent" determined by ICP-AES of the aqueous solutions before and after contact. Final numbers take into account the $3.75: 1$ O:A ratio for both the scrub and strip stages.

***SDS spike was added to each contact individually as opposed to the SDS system where the SDS was added to the simulant together with all other components and allowed to settle before contact with each solvent. 


\subsection{CONCLUSIONS AND RECOMMENDATIONS}

It may be concluded from a combination of ESS testing and qualitative expectations for impurity partitioning that anticipated solvent breakdown products at credible maximum concentrations will not affect the ability of the NG-CSSX solvent's ability to perform effectively. All three anticipated breakdown products of the guanidine suppressor have negligible effect on ESS behavior. Basic decomposition products would be expected to readily wash out of the solvent. Neutral decomposition products such as $N, N^{\prime}$-dicyclohexylurea may build up in the solvent but likely not to a level sufficient to have an adverse effect. Likewise, the effect of 4-sec-butylphenol, the major decomposition product of the Cs-7SB modifier, is expected to be negligible, posing no risk for long-term operation. This phenol can impair stripping at or above $10 \mathrm{mM}$ in the solvent, but its production rate is expected to be low, and it can be expected to wash out in the extraction, scrub, and wash sections of the flowsheet, assuming it behaves in the manner found for BOBCalixC6 $\mathrm{CSSX}^{7}$. It has also been confirmed that the organic phosphates, present in the tank waste, will also be effectively removed from the solvent upon scrubbing and, hence, do not show any adverse results in ESS testing.

It is recommended that future work be conducted to identify the actual breakdown products of the solvent and to measure both their partition ratios in the flowsheet and effects on process performance. From the partitioning ratios, a suitable wash solution can then be recommended and tested to mitigate the inevitable breakdown of solvent components over time. Restoration of the solvent by the washing stage should be demonstrated with degraded solvent. The present positive results reflect only anticipated decomposition products and maximum concentration levels, considering only the suppressor and modifier components of the solvent. The identities of actual decomposition products and their effects are unknown. Decomposition of MaxCalix, completely neglected in this analysis, remains to be considered. Reliable long-term performance of the NG-CSSX solvent depends upon an improved knowledge of solvent decomposition rate and of the identities and fate of the decomposition products. 


\section{EFFECT OF RADIOLYTIC AND THERMAL TREATMENT ON THE PERFORMANCE OF THE NG-CSSX SOLVENT}

\subsection{INTRODUCTION}

While initial test results have demonstrated markedly enhanced performance in most respects, the process will only be practical to the extent that the NG-CSSX solvent is stable to chemical and radiolytic degradation. Thus, the need exists in the development of the NG-CSSX process to ensure the stability of the solvent.

In the development of the original BOBCalixC6 CSSX process, an experimental basis was established for the expectation that the solvent will remain fully functional for at least a year with provision for solvent washing and periodic makeup of solvent components ${ }^{7}$. Dose calculations projected an annual absorbed dose of only $73 \mathrm{krad}(0.73 \mathrm{kiloGray}[\mathrm{kGy}])$, taking into account equipment geometry and configuration similar to that being deployed in the SRS SWPF and the expected levels of ${ }^{137} \mathrm{Cs}$ in the solvent and aqueous phases in the various stages of the process ${ }^{7 \text { Error! Bookmark not defined., }}{ }^{37}$. The maximum sustained temperature was assumed to be $35{ }^{\circ} \mathrm{C}$, limited to the strip section only. Experiments were performed under conditions greatly exceeding the expected stresses to the solvent, both to assess the point and mode of failure and to enable a determination of the identity and fate of degradation products. The expectation of solvent stability derived from the results has been confirmed by over three years of MCU operation without solvent replacement.

Although the NG-CSSX solvent has features in common with BOBCalixC6 CSSX, the use of a different suppressor and different scrub and strip conditions raises the possibility that NG-CSSX solvent could degrade faster than BOBCalixC6 CSSX solvent or that the breakdown products of the NG-CSSX could be more pernicious than those of the BOBCalixC6 CSSX solvent. Based on the previous data on BOBCalixC6 CSSX solvent degradation ${ }^{7}$, it was not expected that MaxCalix or the modifier Cs-7SB would be prone to significantly faster degradation than the corresponding BOBCalixC6 CSSX components. The major degradation product expected for Cs-7SB is SBP, which has been shown in an earlier report not to be more harmful to NG-CSSX performance than it is to BOBCalixC6 CSSX performance (see Chapter 11). Moreover, SBP is expected to wash out of the solvent into the alkaline effluent streams ${ }^{7}$. On the other hand, the stability of the guanidine DCiTG and its effect on the stability of other solvent components have not been examined previously under conditions relevant to SRS processing, and the effect of boric acid on solvent stability is unknown. 
The objective of the work described in this chapter is to test whether the performance of the NG-CSSX process will remain within acceptable limits at least one year under process conditions. A two-pronged approach has been undertaken, first in assessing performance as a function of the degree of thermal and radiolytic stress and second in measuring the actual degradation of the solvent itself. In this chapter, only the performance of the solvent system will be presented, taking the standard ESS testing protocol as the best means of monitoring solvent degradation.

\subsection{EXPERIMENTAL SECTION}

\subsubsection{Materials}

\subsubsection{Solvent Components}

Solvent components were obtained from commercial sources as described in Chapter 2. Solvents were prepared as described in Chapter 2 and the solvent composition can be found in Table 2.2.

\subsubsection{Aqueous Solution Preparation}

The SRS-15 simulant, designed to represent the average SRS tank-waste composition ${ }^{9}$, was prepared according to a method described previously ${ }^{38}$ (Table 2.3).

\subsection{METHODS}

\subsubsection{Radiolytic Treatment}

\subsubsection{Solvent preparation}

Solvent comprised of MaxCalix (0.05 M), Cs-7SB modifier (0.5 M), and DCiTG-M2 guanidine (0.003 M) in Isopar $^{\circledR} \mathrm{L}$ was used for the radiation-treatment studies. The solvent was prepared and washed according to the protocol outlined in Chapter 2, Section 2.2.2.1.

\subsubsection{Pre-irradiation treatment}

Samples of NG-CSSX solvent were irradiated in contact with a) the SRS-15 simulant (not spiked with ${ }^{137} \mathrm{Cs}$ ), b) $25 \mathrm{mM} \mathrm{NaOH}$ scrub solution, and c) $10 \mathrm{mM} \mathrm{H}_{3} \mathrm{BO}_{3}$ strip solution. In order to accurately mimic the state of the solvent in actual extraction, scrub, and strip stages in the process, during each irradiation test the solvents were precontacted as described in Table 12.1. The solvent and aqueous phases were precontacted in polypropylene microtubes mounted by clips on a disk rotated for $60 \mathrm{~min}$. for extractions and $45 \mathrm{~min}$. for scrubs and strips. The solutions were contacted inside an air box maintained at a 
temperature of $25 \pm 0.2{ }^{\circ} \mathrm{C}$. After the contacting period, the tubes were centrifuged for $5 \mathrm{~min}$. at 3000 RPM at the experimental temperature. Due to volume limitations in the ${ }^{60}$ Co source, the 1:4 O:A ratio appropriate for the process could not be accommodated. Rather, a precontact with SRS-15 simulant at $\mathrm{O}: \mathrm{A}=1: 3.5$ was employed so that the loading of the solvent would be approximately the same during the irradiation contact. For the scrub and strip contacts, process O:A ratios were used, 3.75:1. Each sample was contacted for $60 \mathrm{~min}$. inside an air box maintained at a temperature of $25 \pm 0.2{ }^{\circ} \mathrm{C}$ prior to irradiation to ensure the system had reached equilibrium.

Table 12.1. Solvent treatments prior to irradiation

\begin{tabular}{lcccc}
\hline \multicolumn{1}{c}{ Stage } & $\begin{array}{c}\text { Contact solution } \\
\text { during irradiation }\end{array}$ & $\begin{array}{c}\text { Contact O:A ratio } \\
\text { during irradiation }\end{array}$ & Solvent precontacts & $\begin{array}{c}\text { Precontact O:A } \\
\text { ratio }\end{array}$ \\
\hline Extract & SRS-15 simulant & $2: 1$ & SRS-15 simulant & $1: 3.5$ \\
Scrub & $25 \mathrm{mM} \mathrm{NaOH}$ & $3.75: 1$ & SRS-15 simulant & $1: 4$ \\
Strip & $10 \mathrm{mM} \mathrm{Boric} \mathrm{acid}$ & $3.75: 1$ & SRS-15 simulant, & $1: 4$ \\
& & & $2 \times \mathrm{NaOH}(25 \mathrm{mM})$ & $3.75: 1$ \\
\hline
\end{tabular}

\subsubsection{Irradiation}

Radiolytic treatment of the NG-CSSX solvent was carried out using a ${ }^{60} \mathrm{Co}$ source. The samples were contained in sealed $250 \mathrm{~mL}$ Teflon ${ }^{\circledR}$-FEP containers and continuously mixed using an air-driven magnetic stirrer plate. After each level of irradiation, a sample of the contacted solvent and the aqueous layer were removed for ESS testing and analysis, whilst maintaining a constant O:A ratio of the sample undergoing further irradiation.

\subsubsection{Absorbed dose calculation}

The solvent irradiations took place in a J. L. Shepherd Co-60 Irradiator (Model \# 7810-0109-R). The original activity of the source was $24,000 \mathrm{Ci}$ in 1977 . At the time of installation, the dose rate $\left(D_{0}\right)$ was determined to be $18,500 \mathrm{~Gy} / \mathrm{h}$. The half Life $\left(t_{1 / 2}\right)$ of ${ }^{60} \mathrm{Co}$ is $5.27 \mathrm{y}$, which is used to calculate the current dose rate. Using Eq. $1^{39}$, the dose rate was calculated to be approximately $229 \mathrm{~Gy} / \mathrm{h}$. Samples were analyzed at 2500,5000,10,000, 25,000, and 50,000 Gy. The length of irradiations was calculated by simply dividing the desired dose by the dose rate.

$$
D=D_{0} e^{-0.693 t / t_{1 / 2}}
$$

Verification of the dose rate was confirmed with a Fricke dosimeter ${ }^{40,41}$, also called a ferrous sulfate 
dosimeter. It is a chemical dosimeter that determines the oxidation of ferrous ions to ferric ions by ionizing radiation. A spectrophotometer is used to detect the increase in concentration of the ferric ions. The absorbed dose can be calculated from the spectrophotometer data as shown by Eq. $2^{41}$, where $D$ is the absorbed dose to the Fricke solution (in Gy), $\Delta A$ is the net absorbance at the optimum wavelength $(302 \mathrm{~nm}), \rho$ denotes the density of dosimetric solution (in $\mathrm{kg} / \mathrm{m}^{3}$ ), $\varepsilon$ is the molar linear absorption coefficient of the ferric ions $\left(\mathrm{m}^{2} / \mathrm{mol}\right), G$ is the radiation chemical yield of ferric ions (in $\left.\mathrm{mol} / \mathrm{J}\right)$, and $l$ is the optical path length of the dosimetric solution in the cuvette (in $\mathrm{m}$ ). The difference between the calculated dose and the absorbed dose of the Fricke Solution is small and is shown in Table 12.2.

$$
D=\frac{\Delta A}{\varepsilon \times G \times \rho \times l}
$$

Table 12.2. Doses calculated and determined by Fricke dosimetry

\begin{tabular}{ccc}
\hline Calculated Dose (Gy) & Fricke Dose 300 (Gy) & Error (\%) \\
\hline 7.62 & 7.13 & 6 \\
15.2 & 14.1 & 7 \\
38.1 & 34.9 & 8 \\
57.2 & 59.3 & -4 \\
114 & 112 & 2 \\
229 & 230 & -0.5 \\
343 & 347 & -1 \\
\hline
\end{tabular}

For additional confirmation of absorbed dose, a MCNP model of the irradiator was developed at ORNL and used every dimension of the ${ }^{60} \mathrm{Co}$ irradiator and used the dimensions of the Fricke solution. The MCNP model came within 16\% of the calculated dose using Eq. 12.2, which was then deemed acceptable to use as the correct absorbed dose rate for the solvent irradiation calculations. Table 12.3 gives the determined duration of contact for each of the desired doses.

Table 12.3. Defined doses and calculated exposure times

\begin{tabular}{lccccc}
\hline Dose (kGy) & 2.5 & 5 & 10 & 25 & 50 \\
Contact duration $(\mathrm{h})$ & 11 & 22 & 44 & 110 & 220 \\
\hline
\end{tabular}




\subsubsection{Postirradiation treatment}

After irradiation, each sample was cycled through the remaining steps of the "cold" $\mathrm{ES}_{2} \mathrm{~S}_{3}$ cycle followed by a wash step as indicated in Table 12.4. This was done to ensure each solvent was in the same state prior to the "hot" ESS with ${ }^{137} \mathrm{Cs}$ tracer.

Table 12.4. Solvent Treatments following irradiation

\begin{tabular}{|c|c|c|c|c|}
\hline Stage & Contact solution & $\begin{array}{l}\text { Contact } \\
\text { O:A ratio }\end{array}$ & $\begin{array}{c}\text { Solvent } \\
\text { postcontacts }\end{array}$ & $\begin{array}{l}\text { Postcontact } \\
\text { O:A ratio }\end{array}$ \\
\hline Extract & SRS-15 simulant & $2: 1$ & $\begin{array}{c}2 \times \mathrm{NaOH}(25 \mathrm{mM}) \\
3 \times \mathrm{H}_{3} \mathrm{BO}_{3}(10 \mathrm{mM}) \\
\mathrm{NaOH}(10 \mathrm{mM})\end{array}$ & $\begin{array}{l}3.75: 1 \\
3.75: 1 \\
3.75: 1\end{array}$ \\
\hline Scrub & $25 \mathrm{mM} \mathrm{NaOH}$ & $3.75: 1$ & $\begin{array}{c}\mathrm{NaOH}(25 \mathrm{mM}) \\
3 \times \mathrm{H}_{3} \mathrm{BO}_{3}(10 \mathrm{mM}) \\
\mathrm{NaOH}(10 \mathrm{mM})\end{array}$ & $\begin{array}{l}3.75: 1 \\
3.75: 1 \\
3.75: 1\end{array}$ \\
\hline Strip & $10 \mathrm{mM}$ Boric acid & $3.75: 1$ & $\begin{array}{c}2 \times \mathrm{H}_{3} \mathrm{BO}_{3}(10 \mathrm{mM}) \\
\quad \mathrm{NaOH}(10 \mathrm{mM})\end{array}$ & $\begin{array}{l}3.75: 1 \\
3.75: 1\end{array}$ \\
\hline
\end{tabular}

\subsubsection{Thermal Treatment}

\subsubsection{Solvent preparation}

Solvent comprised of 0.05 M MaxCalix, 0.5 M Cs-7SB modifier, and 0.003 M DCiTG-E guanidine in Isopar L was prepared. The solvent was prepared and washed according to the protocol outlined in Chapter 2, Section 2.2.2.1.

Samples of NG-CSSX solvent were thermally treated in contact with a) the SRS-15 simulant (not spiked with ${ }^{137} \mathrm{Cs}$ ), b) $25 \mathrm{mM} \mathrm{NaOH}$ scrub solution, and c) $10 \mathrm{mM} \mathrm{H}_{3} \mathrm{BO}_{3}$ strip solution. In order to accurately mimic the likely state of the solvent in the process, the solvents were precontacted as described in Table 12.5. Each sample was contacted for $60 \mathrm{~min}$. inside an air box maintained at a temperature of $25 \pm 0.2^{\circ} \mathrm{C}$ prior to thermal treatment to ensure the system had reached equilibrium.

Table 12.5. Solvent contacts prior to, and during, thermal treatment

\begin{tabular}{lcccc}
\hline \multicolumn{1}{c}{ Sample } & $\begin{array}{c}\text { Contact solution } \\
\text { during treatment }\end{array}$ & $\begin{array}{c}\text { Contact } \\
\text { O:A ratio }\end{array}$ & $\begin{array}{c}\text { Solvent } \\
\text { precontacts }\end{array}$ & $\begin{array}{c}\text { Precontact } \\
\text { O:A ratio }\end{array}$ \\
\hline Extract & SRS-15 simulant & $4: 1$ & None & None \\
Scrub & $25 \mathrm{mM} \mathrm{NaOH}$ & $3.75: 1$ & SRS-15 simulant & $1: 4$ \\
Strip & $10 \mathrm{mM} \mathrm{Boric} \mathrm{acid}$ & $3.75: 1$ & SRS-15 simulant, & $1: 4$ \\
& & & $2 \times \mathrm{NaOH}(25 \mathrm{mM})$ & $3.75: 1$ \\
\hline
\end{tabular}




\subsubsection{Thermal treatment}

Thermal treatment of the NG-CSSX solvent was carried out in a Lab Line Imperial III Incubator at a constant temperature of $35.0 \pm 0.5^{\circ} \mathrm{C}$ (as determined by biweekly temperature monitoring). The samples were contained in sealed Teflon-FEP containers, using Teflon (pipe thread) tape to avoid significant solvent loss over time. The containers were mounted on a disk, which was then continuously rotated in the incubator. The initial masses of the sealed samples were taken, and any sample loss was determined via mass difference (see below). To minimize sample loss, the Teflon-FEP containers were regularly checked and tightened if necessary.

\subsubsection{Solvent Loss Due to Leakage}

To determine physical sample loss through leakage, the sample weights were determined before and after contact. Preliminary experiments indicated a problem with loss of the organic phase due to leakage around the cap, likely owing to thermal expansion in the incubator. Surveillance and periodic tightening of the caps reduced this leakage. The small losses observed for each sample are given in Table 12.6. The loss from each sample varied but remained on the order of $1 \mathrm{~mL}$ of solvent at most. This amount corresponds to at most $15 \%$ loss of solvent for the extraction contacts, which contained the least volume of solvent. Given the insensitivity of $D_{\mathrm{Cs}}$ value to O:A in extraction, the minor leakage has no significant effect on the ESS results.

Table 12.6. Calculated sample losses

\begin{tabular}{cccccc}
\hline Sample & 1 Month & 2 Months & 3 Months & 5 Months & 13 Months \\
\hline Extraction 1 & $0.85 \mathrm{~g}$ & $0.65 \mathrm{~g}$ & $0.8 \mathrm{~g}$ & $0.42 \mathrm{~g}$ & $0.15 \mathrm{~g}$ \\
${\text { Extraction } 2^{a}}^{a}$ & $0.48 \mathrm{~g}$ & $0.52 \mathrm{~g}$ & $0.62 \mathrm{~g}$ & $0.04 \mathrm{~g}$ & $0.07 \mathrm{~g}$ \\
Scrub & $0.88 \mathrm{~g}$ & $0.14 \mathrm{~g}$ & $0.04 \mathrm{~g}$ & $0.50 \mathrm{~g}$ & $0.42 \mathrm{~g}$ \\
Strip & $0.03 \mathrm{~g}$ & $0.52 \mathrm{~g}$ & $0.03 \mathrm{~g}$ & $0.06 \mathrm{~g}$ & $0.12 \mathrm{~g}$ \\
\hline
\end{tabular}

${ }^{a}$ In order to get duplicates in ESS testing, a minimum of $14 \mathrm{~mL}$ of solvent was required, due to the O:A of the extraction step being high and the limited volume of the Teflon containers $(45 \mathrm{~mL})$. Two extraction samples were required for each test.

\subsubsection{Post-thermal treatment}

After thermal treatment, each sample was cycled through the remaining steps of the cold $\mathrm{ES}_{2} \mathrm{~S}_{3}$ cycle followed by a wash step as indicated in Table 12.7. The post-thermal treatment was performed in a similar manner as described for the postirradiation treatment in section 11.3.1.5. 


\subsubsection{Cesium Distribution Ratios $\left(D_{\mathrm{Cs}}\right)$}

$D_{\mathrm{Cs}}$ in duplicate $\mathrm{ES}_{2} \mathrm{~S}_{3}$ tests with simulants spiked with ${ }^{137} \mathrm{Cs}$ were run in a manner similar to that described previously ${ }^{7}$ (see also Chapter 2), with one extraction stage followed by two scrub stages using $0.025 \mathrm{M} \mathrm{NaOH}$, and three strip stages with $0.010 \mathrm{M} \mathrm{H}_{3} \mathrm{BO}_{3}$.

Table 12.7. Solvent contacts following thermal treatment

\begin{tabular}{|c|c|c|c|c|}
\hline Sample & Contact solution & $\begin{array}{c}\text { Contact } \\
\text { O:A ratio }\end{array}$ & $\begin{array}{c}\text { Solvent } \\
\text { postcontacts }\end{array}$ & $\begin{array}{c}\text { Postcontact } \\
\text { O:A ratio }\end{array}$ \\
\hline Extract & SRS-15 simulant & $4: 1$ & $\begin{array}{c}2 \times \mathrm{NaOH}(25 \mathrm{mM}) \\
3 \times \mathrm{H}_{3} \mathrm{BO}_{3}(10 \mathrm{mM}) \\
\mathrm{NaOH}(10 \mathrm{mM})\end{array}$ & $\begin{array}{l}3.75: 1 \\
3.75: 1 \\
3.75: 1\end{array}$ \\
\hline Scrub & $25 \mathrm{mM} \mathrm{NaOH}$ & $3.75: 1$ & $\begin{array}{c}\mathrm{NaOH}(25 \mathrm{mM}) \\
3 \times \mathrm{H}_{3} \mathrm{BO}_{3}(10 \mathrm{mM}) \\
\mathrm{NaOH}(10 \mathrm{mM})\end{array}$ & $\begin{array}{l}3.75: 1 \\
3.75: 1 \\
3.75: 1\end{array}$ \\
\hline Strip & $10 \mathrm{mM}$ Boric acid & $3.75: 1$ & $\begin{array}{c}2 \times \mathrm{H}_{3} \mathrm{BO}_{3}(10 \mathrm{mM}) \\
\quad \mathrm{NaOH}(10 \mathrm{mM})\end{array}$ & $\begin{array}{l}3.75: 1 \\
3.75: 1\end{array}$ \\
\hline
\end{tabular}

Based upon the agreement of duplicate samples run within the same set of measurements, the precision of $D_{\mathrm{Cs}}$ values within an experiment has generally been found to worsen in the steps of an ESS as follows: $\pm 5 \%$ (extraction and scrub), $\pm 10 \%$ (first strip), and $\pm 30 \%$ (second and third strips). This duplicate precision correlates with volumetric precision ( $\pm 3 \%$ ) and counting precision, which is approximately $\pm 3 \%$ (extraction), $\pm 1 \%$ (scrub), and $\pm 30-50 \%$ (strip). Owing to the temperature sensitivity of cesium distribution (ca. $10 \%$ change in $D_{\mathrm{Cs}}$ per ${ }^{\circ} \mathrm{C}^{7}$ ), sample handling can introduce additional error. Thus, effective overall precision of extraction, scrub, and first-strip $D_{\mathrm{Cs}}$ values is estimated to be on the order of $\pm 10 \%$. Each value presented in the tables in this report is the average of two duplicates; the error given represents the standard deviation of the duplicates, the parenthetic number referring to the precision of the corresponding previous digit or, in a few cases, two digits (e.g., 2.11(3) $\times 10^{-1}$ means $0.211 \pm 0.003$ and $3.5(15) \times 10^{-4}$ means $\left.0.00035 \pm 0.00015\right)$.

\subsection{RESULTS AND DISCUSSION}

\subsubsection{Reference Limits}

Reference limits are needed for evaluating ESS performance with the purpose of identifying conditions under which the chemistry is not functioning as intended. An excursion outside of the reference limits thus indicates an additional factor, introduced by experimental conditions, which could eventually lead to process upset, especially if the trend away from the reference limit continues over time. It is thus a "caution light." For standard $\mathrm{ES}_{2} \mathrm{~S}_{3}$ tests carried out at $25{ }^{\circ} \mathrm{C}$, the following reference limits have been 
selected:

$$
\begin{gathered}
D_{\mathrm{Cs}} \geq 40 \text { for extraction, } \\
0.5 \leq D_{\mathrm{Cs}} \leq 10 \text { for scrubbing, } \\
D_{\mathrm{Cs}} \leq 0.1 \text { for stripping. }
\end{gathered}
$$

Actual process $D_{C s}$ tolerances corresponding to probable process upset depend on feed conditions, equipment design, and site requirements and thus have to be set for a given flowsheet. It should therefore be kept in mind that the above selected limits are reference points that do not necessarily indicate that the performance has deteriorated anywhere near the level of process upset. In fact, multistage centrifugalcontactor flowsheet testing on simulated and real SRS high Level salt waste operated with consistent DF values an order of magnitude above the target performance despite having some scrub and strip stages apparently operating outside of the reference $D_{C S}$ limits given above ${ }^{41}$.

Extraction performance depends strongly on feed composition, particularly the potassium concentration ${ }^{30}$, as discussed in Chapter 8.4. In this study, only the SRS-15 simulant, having a potassium concentration of $0.015 \mathrm{M}$, was employed in order to reduce the number of variables. At the SRS, the bounding concentration of potassium is $0.045 \mathrm{M}^{7}$, which depresses the $D_{C s}$ value noticeably, ca. $20 \%$ to a value of 41 at $25^{\circ} \mathrm{C}^{6}$. With its many stages, the SWPF would be less impacted by a decrease in extraction $D_{\mathrm{Cs}}$ value than the MCU. A value of 40 could likely be accommodated at the MCU, which could be increased if needed by decreasing the temperature of the extraction section ${ }^{7}$ (see Chapter 8).

The scrub reference limits $0.5 \leq D_{\mathrm{Cs}} \leq 10$ are not critical in themselves, but rather they indicate potential impact to extraction and stripping as follows. If the scrub $D_{\mathrm{Cs}}$ falls much below 0.5 , a significant cesium recycle back to extraction will occur depending on the scrub O:A used (assumed to be at least 3.75:1). If the scrub $D_{\mathrm{Cs}}$ value exceeds 10 , a significant amount of potassium may start to report to the stripping section, impairing its performance. Scrubbing performance, particularly the first scrub, can vary somewhat with waste feed, but stripping should perform approximately independently of the feed composition because the scrub section prepares the solvent so it always enters the strip section in the same chemical state. 


\subsection{RADIOLYTIC TREATMENT}

\subsubsection{General Observations}

Effects of radiation on the solvent are evident in solvent discoloration and impairment of ESS performance. However, it is shown based on the results presented below that, for the irradiated NG-CSSX in contact with SRS-15 simulant, $25 \mathrm{mM} \mathrm{NaOH}$, and $10 \mathrm{mM} \mathrm{H}_{3} \mathrm{BO}_{3}$, the $D_{\mathrm{Cs}}$ values in batch ESS tests are not sufficiently affected to impair processing until the contacted solutions are exposed to more than $10 \mathrm{kGy}$. An annual radiation dose to the solvent for a facility configuration similar to the SWPF was estimated to be $0.73 \mathrm{kGy}^{7,37}$. Assuming that the MCU will process feeds having a maximum ${ }^{137} \mathrm{Cs}$ activity that is $1 / 6$ that of the maximum ${ }^{137} \mathrm{Cs}$ activity of the SWPF, the annual dose to the solvent at the MCU is expected to be on the order of only $0.12 \mathrm{kGy}$. On average, Hanford feeds would have a lower ${ }^{137} \mathrm{Cs}$ activity than those at the SRS. Thus, the expected effect of radiation on NG-CSSX performance in anticipated tank-waste related applications is negligible. A careful examination of the data below reveals what parts of the process undergo the most degradation and what parts of the process are most affected by the degradation.

\subsubsection{Coloration of Irradiated Solvent}

The effect of irradiation on the color of the NG-CSSX solvent is shown in Fig. 12.1. Irradiation in the presence of SRS-15 simulant gave the largest degree of discoloration, with the solvent developing a dark amber hue after $50 \mathrm{kGy}$. Discoloration of the irradiated solvent in contact with the $25 \mathrm{mM} \mathrm{NaOH}$ scrub solution was similar to that of solvent in contact with the SRS-15 simulant but to a lesser extent. In contrast, the irradiated solvent in contact with the $10 \mathrm{mM} \mathrm{H}_{3} \mathrm{BO}_{3}$ strip solution showed little to no color change with increased dose. It would appear, from solvent discoloration, that the solvent is less radiolytically stable under basic conditions. From the ESS data tabulated below, it will be seen that the degree of coloration correlates with the effect on cesium distribution behavior. 


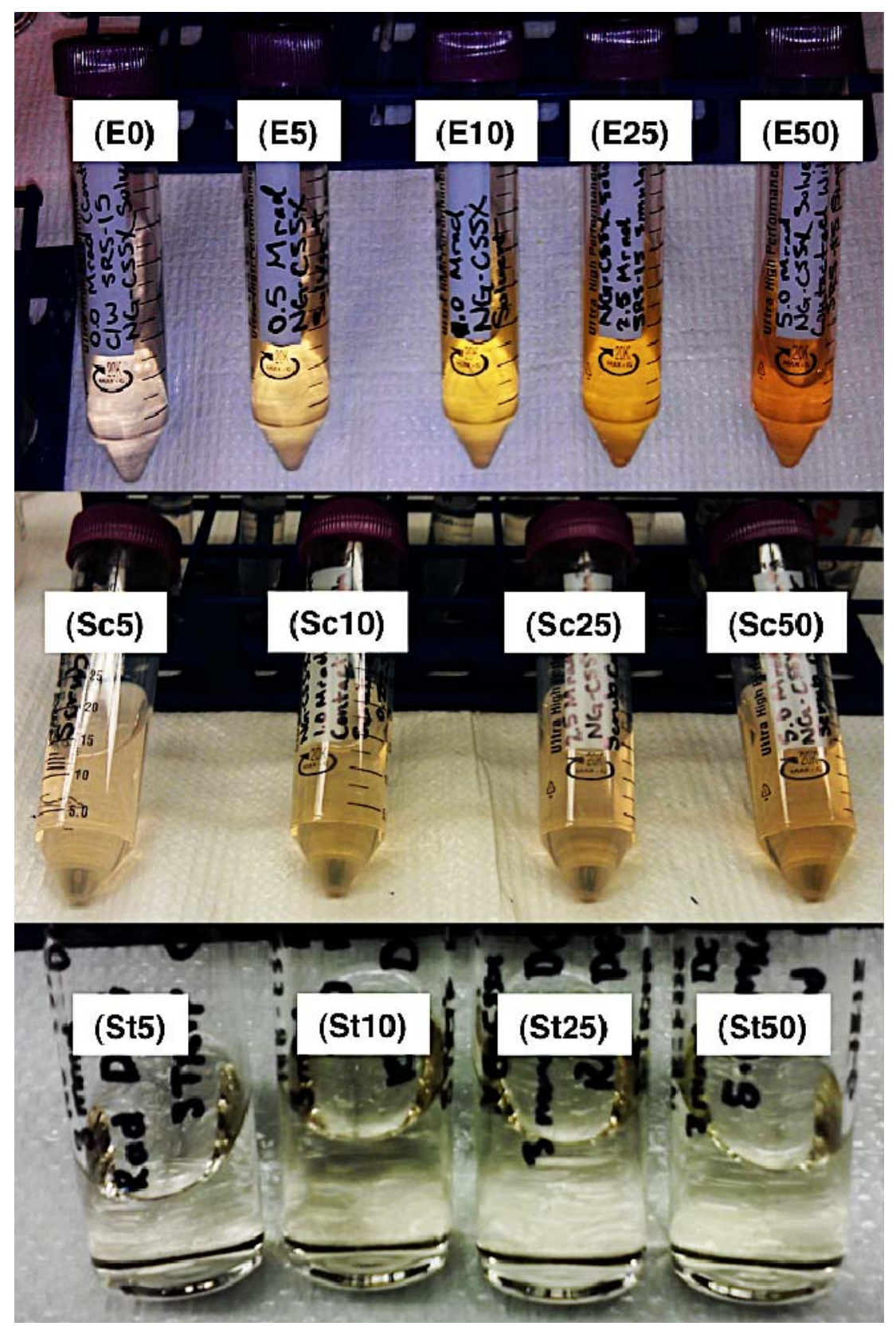

Fig. 12.1. NG-CSSX solvent samples after irradiation in contact with SRS-15 simulant (E series), 25 mM $\mathrm{NaOH}$ (Sc series), and $10 \mathrm{mM} \mathrm{H}_{3} \mathrm{BO}_{3}$ (St series). Figure numbers denote the absorbed dose in kGy.

\subsubsection{Irradiation During Contact with SRS-15 Simulant}

Irradiation in the presence of SRS-15 simulant proved to have the strongest effect of the three aqueous phases tested. The $D_{\mathrm{Cs}}$ values for the $\mathrm{ES}_{2} \mathrm{~S}_{3}$ test of the irradiated NG-CSSX solvent whilst in contact with cold SRS-15 simulant are given in Table 12.8. It was found that the $D_{\mathrm{Cs}}$ values for extraction vary little with increased irradiation, with an average $D_{\mathrm{Cs}}$ of $61 \pm 2$. There is a slight upward trend, possibly real if one supposes an effect of anionic degradation products, but the trend is not statistically significant. The 
$D_{\mathrm{Cs}}$ values for scrubbing definitely creep upward, reaching the reference limit of 10 at $10 \mathrm{kGy}$. Also, the $D_{\mathrm{Cs}}$ value of the second scrub overtakes that of the first after $5 \mathrm{kGy}$, which can be interpreted as a residual effect of an anionic degradation product that is not scrubbed out in the first scrub contact. The second scrub should be more sensitive to anionic impurities than the first scrub, as the effect is applied to a lower concentration of cations, principally cesium, as sodium and potassium should be mostly removed in the first scrub. The $D_{\mathrm{Cs}}$ values for stripping also increase with increased exposure and at $21 \mathrm{kGy}$ reach the reference value of 0.1 . At $50 \mathrm{kGy}$ the stripping fails catastrophically, shown by $D_{\mathrm{Cs}}$ values exceeding unity and increasing on consecutive stripping stages.

Table 12.8. $D_{\mathrm{Cs}}$ for ESS batch tests ${ }^{\text {a }}$ following radiolytic treatment of NG-CSSX solvent in contact with SRS-15 simulant at $\mathrm{O}: \mathrm{A}=\mathbf{2 : 1}$

\begin{tabular}{llllll}
\hline \multicolumn{1}{c}{ Stage } & \multicolumn{1}{c}{$\mathbf{0}$} & \multicolumn{1}{c}{$\mathbf{5} \mathbf{~ k G y}$} & \multicolumn{1}{c}{$\mathbf{1 0} \mathbf{~ k G y}$} & \multicolumn{1}{c}{$\mathbf{2 5} \mathbf{~ k G y}$} & $\mathbf{5 0} \mathbf{k G y}$ \\
\hline Extraction & $59.0(3)$ & $58.5(1)$ & $62(1)$ & $61.3(1)$ & $63(2)$ \\
Scrub 1 & $2.5(1)$ & $6.3(3)$ & $7.9(5)$ & $12(1)$ & $18(3)$ \\
Scrub 2 & $1.70(0)$ & $6.25(5)$ & $10.4(7)$ & $21.7(2)$ & $29(1)$ \\
Strip 1 & $2.54(9) \times 10^{-3}$ & $1.1(2) \times 10^{-2}$ & $2.77(7) \times 10^{-2}$ & $1.4(1) \times 10^{-1}$ & $1.320(0)$ \\
Strip 2 & $2.0(2) \times 10^{-4}$ & $1.1(2) \times 10^{-3}$ & $2.8(2) \times 10^{-3}$ & $1.8(2) \times 10^{-2}$ & $1.6(1)$ \\
Strip 3 & $1.7(12) \times 10^{-4}$ & $9(4) \times 10^{-4}$ & $1.10(3) \times 10^{-3}$ & $5.4(3) \times 10^{-3}$ & $1.9(2)$ \\
\hline
\end{tabular}

${ }^{a}$ The aqueous phases were SRS-15 waste simulant for extraction, $0.025 \mathrm{M} \mathrm{NaOH}$ for the scrub stages, and $0.01 \mathrm{M} \mathrm{H}_{3} \mathrm{BO}_{3}$ for the strip stages. Solvent composition is defined in Table 2.2. The O:A ratios in the test ESS were 1:4 for extraction and 3.75:1 for both scrubbing and stripping (extraction O:A was 2:1 in irradiation). ESS experiments were carried out at a constant temperature of $25 \pm 0.2^{\circ} \mathrm{C}$.

\subsubsection{Irradiation During Contact with $25 \mathrm{mM} \mathrm{NaOH}$}

Behavior of the irradiated solvent in contact with scrub solution was similar to that of solvent in contact with the SRS-15 simulant, though not as severe. The $D_{\mathrm{Cs}}$ values for the $\mathrm{ES}_{2} \mathrm{~S}_{3}$ tests of the irradiated NGCSSX solvent whilst in contact with $25 \mathrm{mM} \mathrm{NaOH}$ are given in Table 12.9. It was found that the $D_{\mathrm{Cs}}$ values for extraction again vary little with increased irradiation with an average of $56 \pm 2$. In this case, the slight trend is downward, again not statistically significant. The values of $D_{\mathrm{Cs}}$ for scrubbing increase, but to a slightly lesser extent than seen for the irradiated solvent in contact with SRS-15 simulant. The scrub reference limit is not reached until $20 \mathrm{kGy}$ (interpolated), and the second scrub does not overtake the first until about $30 \mathrm{kGy}$. The $D_{\mathrm{Cs}}$ values for stripping also increase with increased dose, reaching the reference value at approximately $34 \mathrm{kGy}$ (interpolated), though still with consecutive strips each performing better than the previous strip. 
Table 12.9. $D_{\mathrm{Cs}}$ for $E S S$ batch tests ${ }^{\text {a }}$ following radiolytic treatment of NG-CSSX solvent in contact with 25 $\mathrm{mM} \mathrm{NaOH}$ at $\mathrm{O}: \mathrm{A}=3.75: 1$

\begin{tabular}{llllll}
\hline \multicolumn{1}{r}{ Stage } & \multicolumn{1}{c}{$\mathbf{0}$} & \multicolumn{1}{c}{$\mathbf{5} \mathbf{~ k G y}$} & $\mathbf{1 0} \mathbf{~ k G y}$ & \multicolumn{1}{c}{$\mathbf{2 5} \mathbf{~ k G y}$} & $\mathbf{5 0} \mathbf{~ k G y}$ \\
\hline Extraction & $57(1)$ & $56.6(1)$ & $57.2(8)$ & $56.6(9)$ & $54.0(6)$ \\
Scrub 1 & $3.40(9)$ & $5.5(8)$ & $7.80(5)$ & $11(1)$ & $13.6(1)$ \\
Scrub 2 & $1.64(2)$ & $3.6(1)$ & $5.1(2)$ & $9.9(1)$ & $17.0(1)$ \\
Strip 1 & $2.5(2) \times 10^{-3}$ & $5.6(4) \times 10^{-3}$ & $9.9(6) \times 10^{-3}$ & $3.27(9) \times 10^{-2}$ & $2.12(4) \times 10^{-1}$ \\
Strip 2 & $2.10(2) \times 10^{-4}$ & $6(2) \times 10^{-4}$ & $1.11(1) \times 10^{-3}$ & $3.7(4) \times 10^{-3}$ & $7.7(2) \times 10^{-2}$ \\
Strip 3 & $2.6(7) \times 10^{-4}$ & $4(1) \times 10^{-4}$ & $6.8(2) \times 10^{-4}$ & $1.62(4) \times 10^{-3}$ & $2.4(1) \times 10^{-2}$ \\
\hline
\end{tabular}

${ }^{a}$ The aqueous phases were SRS-15 waste simulant for extraction, $0.025 \mathrm{M} \mathrm{NaOH}$ for the scrub stages, and $0.01 \mathrm{M} \mathrm{H}_{3} \mathrm{BO}_{3}$ for the strip stages. Solvent composition is defined in Table 2.2. The O:A ratios in the ESS tests were 1:4 for extraction and 3.75:1 for both scrubbing and stripping. ESS experiments were carried out at a constant temperature of $25 \pm 0.2^{\circ} \mathrm{C}$.

\subsubsection{Irradiation During Contact with $10 \mathrm{mM} \mathrm{H}_{3} \mathrm{BO}_{3}$}

Contact with the strip solution during irradiation affected the ESS performance approximately the same as contact with scrub solution. The $D_{\mathrm{Cs}}$ values for the $\mathrm{ES}_{2} \mathrm{~S}_{3}$ tests are given in Table 12.10. It was found that, when in contact with $10 \mathrm{mM} \mathrm{H}_{3} \mathrm{BO}_{3}$ during irradiation at $\mathrm{O}: \mathrm{A}=3.75: 1$, the $D_{\mathrm{Cs}}$ values on subsequent extraction vary little with increased irradiation with an average of $60 \pm 4$, a noted upward trend not being statistically significant. The $D_{\mathrm{Cs}}$ for scrubbing increased in a similar manner as seen above, reaching the reference limit at $20 \mathrm{kGy}$, with the second scrub overtaking the first scrub. The trend in the $D_{\mathrm{Cs}}$ values for stripping, however, is somewhat different than that observed for irradiation of contacted with SRS-15 simulant and scrub solutions. The $D_{\mathrm{Cs}}$ values for strips 1 and 2 increase with increased exposure, the first strip reaching the reference limit at $32 \mathrm{kGy}$. The $D_{\mathrm{Cs}}$ values for the third strip, however, remained low. This suggests that the NG-CSSX solvent is somewhat more stable to radiolytic degradation under the neutral conditions of stripping.

Table 12.10. $D_{\mathrm{Cs}}$ for ESS batch tests ${ }^{\text {a }}$ following radiolytic treatment of NG-CSSX solvent in contact with $10 \mathrm{mM} \mathrm{H}_{3} \mathrm{BO}_{3}$ at $\mathrm{O}: \mathrm{A}, 3.75: 1$

\begin{tabular}{llcllc}
\hline \multicolumn{1}{c}{ Stage } & \multicolumn{1}{c}{$\mathbf{0}$} & $\mathbf{5 ~ k G y}$ & $\mathbf{1 0} \mathbf{~ k G y}$ & \multicolumn{1}{c}{$\mathbf{2 5} \mathbf{~ k G y}$} & $\mathbf{5 0 ~ k G y}$ \\
\hline Extraction & $56(2)$ & $58.0(7)$ & $58(2)$ & $64(1)$ & $64.7(8)$ \\
Scrub 1 & $3.1(2)$ & $5.6(6)$ & $6.63(1)$ & $11.01(2)$ & $17.4(3)$ \\
Scrub 2 & $1.27(1)$ & $3.8(1)$ & $5.2(2)$ & $12.9(4)$ & $21.9(5)$ \\
Strip 1 & $2.1(1) \times 10^{-3}$ & $4.6(4) \times 10^{-3}$ & $8.5(7) \times 10^{-3}$ & $5.2(3) \times 10^{-2}$ & $3.9(4) \times 10^{-1}$ \\
Strip 2 & $4(2) \times 10^{-4}$ & $6.6(3) \times 10^{-4}$ & $7.5(6) \times 10^{-4}$ & $4.7(2) \times 10^{-3}$ & $1.40(7) \times 10^{-1}$ \\
Strip 3 & $2.7(4) \times 10^{-4}$ & $1.68(7) \times 10^{-3}$ & $6.65(3) \times 10^{-4}$ & $4.2(5) \times 10^{-4}$ & $3.2(4) \times 10^{-4}$ \\
\hline \multicolumn{7}{l}{ The aqueous phases were SRS-15 waste simulant for extraction, $0.025 \mathrm{M} \mathrm{NaOH}$ for the scrub stages, and $0.01 \mathrm{M} \mathrm{H}_{3} \mathrm{BO}_{3}$} \\
for the strip stages. Solvent composition is defined in Chapter 2. The O:A ratios in the ESS tests were $1: 4$ for extraction and \\
3.75:1 for both scrubbing and stripping. Experiments were carried out at a constant temperature of $25 \pm 0.2{ }^{\circ} \mathrm{C}$.
\end{tabular}




\subsubsection{Impact of Radiation on Process Performance}

Based on the above data, the anticipated impact of radiation on process performance is expected to be negligible. All samples irradiated under process conditions perform within adopted reference limits until scrub $D_{\mathrm{Cs}}$ values for solvent irradiated in the presence of SRS-15 simulant exceed a reference value of 10 at $10 \mathrm{kGy}$. If it is assumed conservatively that all of the dose to the solvent is received in the presence of the most severe aqueous phase (SRS-15), then $10 \mathrm{kGy}$ corresponds to a 13 year absorbed dose, using the SWPF basis of $0.73 \mathrm{kGy} / \mathrm{y}^{7,38}$. The post-irradiation ESS behavior of all samples is similar, extraction $D_{\mathrm{Cs}}$ being rather constant, scrubbing $D_{\mathrm{Cs}}$ increasing eventually to above the reference value with the second scrub overtaking the first, and the first and second strip $D_{\mathrm{Cs}}$ increasing, the first eventually exceeding the reference limit. Stripping $D_{\mathrm{Cs}}$ values change most rapidly in all cases, but interestingly, it is the scrub $D_{\mathrm{Cs}}$ values that exceed the reference limit first. Overall, the deterioration of ESS performance is worst when the solvent is irradiated in the presence of the SRS-15 simulant.

It should be noted that the experimental setup for radiolysis is overly conservative, as all radiolysis products are confined to the system throughout the entire irradiation. Under normal operation, degradation products would be continuously washed out of the solvent according to their partitioning to the aqueous process solutions. In the test, chemically reactive species formed by radiolytic degradation of the solvent components remain in the system to either build up or chemically react.

The most likely explanation for the slightly increased scrubbing $D_{\mathrm{Cs}}$ values and increased stripping $D_{\mathrm{Cs}}$ values with increased exposure is the buildup of lipophilic anionic impurities combined with a loss of suppressor capacity to neutralize the effect of the anionic impurities. The following analyses quantify the loss of the suppressor, the buildup of the lipophilic impurity SBP, and the effect these have on the loading of sodium by the irradiated solvent.

\subsubsection{Solvent analysis for degratation}

Irradiation resulted in the increase in sodium capacity of the NG-CSSX solvent. The $D_{\mathrm{Na}}$ values for each of the irradiated solvents after contact with $\mathrm{NaOH}(0.5 \mathrm{M}, \mathrm{O}: \mathrm{A} 1: 1)$ are given in Table 12.11. It was found that irradiation of the solvent for $50 \mathrm{kGy}$ results in an approximate ten-fold increase in the levels of sodium extracted. This increase in sodium loading capability together with the $\mathrm{ES}_{2} \mathrm{~S}_{3}$ data suggests an increase in the presence of lipophilic anions in the irradiated solvents. 
Table 12.11. Sodium distribution ratios for NG-CSSX solvent post radiolytic treatment in contact with $0.5 \mathrm{M} \mathrm{NaOH}$ at $\mathrm{O}: \mathrm{A}, 1: 1$

\begin{tabular}{llllll}
\hline \multicolumn{1}{c}{ Stage } & \multicolumn{1}{c}{$\mathbf{0}$} & \multicolumn{1}{c}{$\mathbf{5} \mathbf{~ k G y}$} & \multicolumn{1}{c}{$\mathbf{1 0} \mathbf{~ k G y}$} & \multicolumn{1}{c}{$\mathbf{2 5} \mathbf{~ k G y}$} & \multicolumn{1}{c}{$\mathbf{5 0} \mathbf{~ k G y}$} \\
\hline Extraction & $1.1(2) \times 10^{-3}$ & $3.0(4) \times 10^{-3}$ & $4.4(4) \times 10^{-3}$ & $7.4(1) \times 10^{-3}$ & $1.2(2) \times 10^{-2}$ \\
Scrub & $9.6(4) \times 10^{-4}$ & $3.9(7) \times 10^{-3}$ & $5(1) \times 10^{-3}$ & $7.8(9) \times 10^{-3}$ & $1.03(3) \times 10^{-2}$ \\
Strip & $1.29(6) \times 10^{-3}$ & $2.8(2) \times 10^{-3}$ & $3.9(6) \times 10^{-3}$ & $6(1) \times 10^{-3}$ & $9(2) \times 10^{-3}$ \\
\hline
\end{tabular}

The solvent irradiated whilst in contact with the extraction aqueous feed showed the largest sodium uptake after $5 \mathrm{MRad}$, but those in contact with the scrub and strip solutions also showed a significant increase.

\subsubsection{Mass Spec Analysis of NG-CSSX Solvent after Irradiation in Contact with SRS-15}

As the initial concentration of cesium in the SRS-15 simulant is known, and its distribution has been determined for each level of irradiation, the cesium concentration in the solvent after extraction remains constant at $5.50(2) \times 10^{-4} \mathrm{M}$. By using the $[\text { MaxCalix-Cs] }]^{+}$peak as an internal standard, mass spectrometry can be used to monitor change in the speciation occurring, albeit qualitative, before and after irradiation.

Fig. 12.2 shows the three major observable species in the electrospray ionization mass spectrometry (ESIMS) of the NG-CSSX solvent before and after irradiation whilst in contact with the SRS-15 simulant to be $[\text { MaxCalix-Cs }]^{+},[\text {MaxCalix-K }]^{+}$and $[\mathrm{DCiTG}-\mathrm{H}]^{+}$, all present in the spectrum due to their inherent ionic nature. There are distinct changes in the relative intensities of the $[\text { MaxCalix-K }]^{+}$and $[\mathrm{DCiTG}-\mathrm{H}]^{+}$ cations upon irradiation. The relative increase in $[\mathrm{MaxCalix}-\mathrm{K}]^{+}$intensity gives further weight to the argument that lipophilic anions are formed upon irradiation, facilitating the extraction of potassium and resulting in the impairment of the scrubbing $D_{C s}$ values observed. The reduction in peak intensity of the $[\text { DCiTG-H }]^{+}$peak confirms the observed reduction in suppressor concentration upon irradiation, thought to be one of the causes of the increased stripping $D_{C s}$ values. 


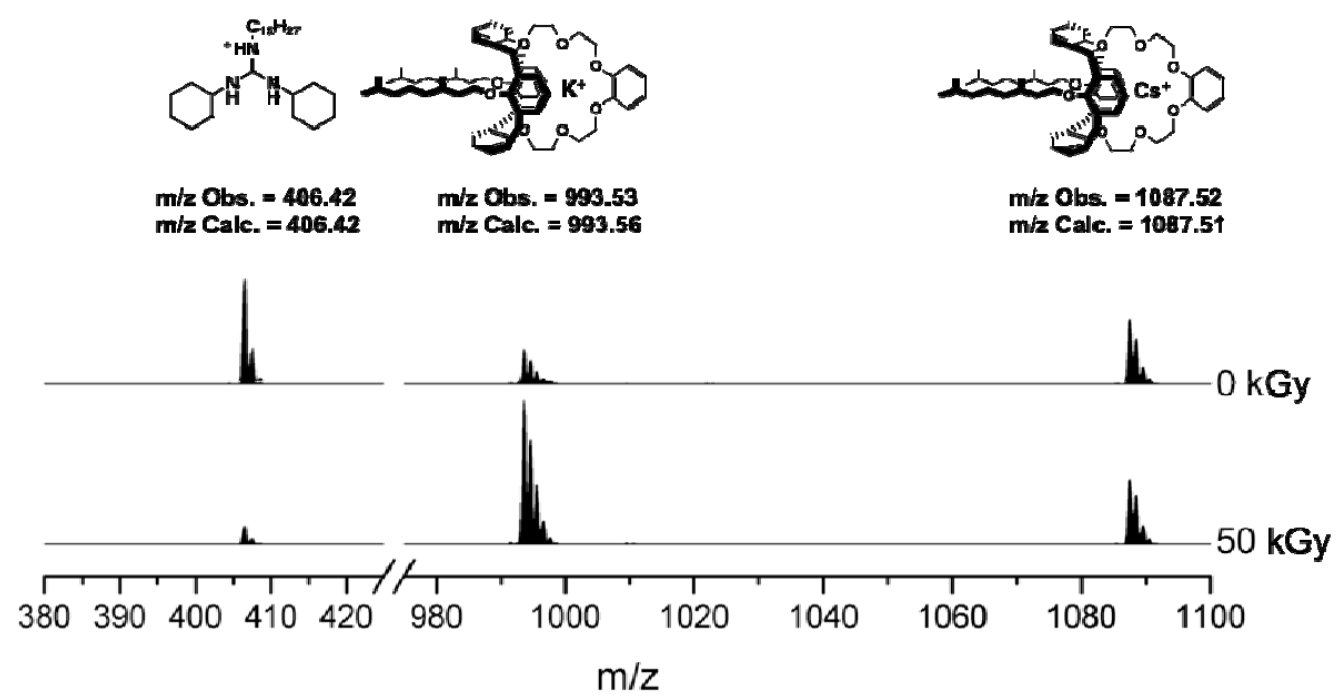

Fig. 12.2. Partial mass spectra of the NG-CSSX solvent pre- and postirradiation whilst in contact with SRS-15.

\subsubsection{GC Quantification for the degradation of the DCiTGnidine suppressor}

GC was successfully used to quantify the degradation of the guanidine suppressor. The results from the GC analysis are presented in Table 12.12, the solvent irradiated whilst in contact with the waste simulant showed the largest reduction in suppressor concentration of around $50 \%$.

As described in more detail in Chapter 3, under pristine solvent conditions the minimum suppressor concentration with which the solvent can function is $0.25 \mathrm{mM}^{27}$. This indicates that the deviation of the $D_{C S}$ values shown in the ESS testing is the result of more than one process, as the guanidine levels are still more than enough for the solvent to function, were it pristine. The formation of species due to the degradation of solvent components must also be occurring.

Table 12.12 The DCiTG suppressor concentration before and after solvent irradiation

\begin{tabular}{llllcc}
\hline \multicolumn{1}{c}{ Stage } & 0 MRad & 0.5 MRad & 1.0 MRad & 2.5 MRad & 5.0 MRad \\
\hline Extraction & $2.9(1)$ & $2.6(1)$ & $2.3(1)$ & $1.8(1)$ & $1.4(1)$ \\
Scrub & $3.1(2)$ & $2.4(1)$ & $2.2(1)$ & $2.0(1)$ & $1.9(1)$ \\
Strip & $3.0(2)$ & $2.7(1)$ & $2.4(1)$ & $2.2(1)$ & $2.1(1)$ \\
Control $^{\mathrm{a}}$ & $2.9(1)$ & $2.8(1)$ & $2.6(1)$ & $2.4(1)$ & $2.0(1)$ \\
\hline
\end{tabular}

The control solvent was a sample of NG-CSSX solvent that had been undergone the solvent wash stages but was irradiated

${ }^{\mathrm{a}}$ The control solvent was irradiated whilst not in contact with any aqueous feed.

The error given is the precision of the measurement determined to be $5 \%$; the maximum replicate error of the external calibration (carried out in triplicate, see ESI). 


\subsubsection{Degradation product identification}

Quantitative and qualitative investigations have been carried out in an attempt to identify some of the radiolytic degradation products and their possible influence on the efficacy of the NG-CSSX solvent.

\subsubsection{Degradation of the Cs-7SB Modifier}

The concentration of SBP in each of the irradiated solvents was determined using GC. As can be seen in Table 12.13, the level of SBP in the irradiated solvents increases with dose, and that solvent in contact with basic SRS-15 and $\mathrm{NaOH}$ solutions was found to contain higher levels. If the presence of SBP is directly related to the loss of Cs-7SB due to radiolytic breakdown, then the presence of $2.72 \mathrm{mM}$ of SBP after $5 \mathrm{MRad}$ is equivalent to be less than $0.01 \%$ loss per annual dose at SWPF.

Table 12.13 The sec-butylphenol concentration (mM) in the NG-CSSX solvent after irradiation as determined via GC

\begin{tabular}{lllccc}
\hline \multicolumn{1}{c}{ Stage } & 0 MRad & 0.5 MRad & 1.0 MRad & 2.5 MRad & 5.0 MRad \\
\hline Extraction & $0.06(1)$ & $0.85(1)$ & $1.71(7)$ & $2.39(3)$ & $2.72(4)$ \\
Scrub & $0.14(1)$ & $0.61(3)$ & $0.89(2)$ & $1.53(2)$ & $1.89(7)$ \\
Strip & $0.08(1)$ & $0.27(1)$ & $0.56(2)$ & $0.65(1)$ & $0.99(3)$ \\
\hline
\end{tabular}

The control solvent was a sample of NG-CSSX solvent that had been undergone the solvent wash stages but was not irradiated.

\subsubsection{ESI-MS of partitioned degradation products in strip feed}

ESI-MS was used to further analyze for breakdown products that could potentially have partitioned out of the solvent in the scrubbing stages. The first $\mathrm{NaOH}$ scrub solution after contact with the NG-CSSX solvent that had been irradiated (50 MRad) in contact with SRS-15 was analyzed qualitatively. Table 12.14 lists the $\mathrm{m} / \mathrm{z}$ for the peaks observed, the possible origin and the predicted $\mathrm{m} / \mathrm{z}$ for the proposed species. The majority of the species identified were clusters of sodium and potassium salts of the Cs-7SB modifier $\left(\left[((\mathrm{Mod}-\mathrm{H}) \mathrm{M})_{\mathrm{n}} \mathrm{M}\right]^{+}\right.$, where $\left.\mathrm{M}=\mathrm{Na}, \mathrm{K}\right)$, but the suppressor ([DCiTG-H $\left.]^{+}\right)$was also identified. More interestingly a species containing the extractant, $\left[\left(\left(\text { MaxCalix }-\mathrm{C}_{10} \mathrm{H}_{21}\right) \mathrm{Na}\right) \mathrm{K}\right]^{+}(\mathrm{m} / \mathrm{z}=875.5)$, was also identified, indicating that the extractant MaxCalix can radiolytically cleave in the same manner as observed for the Cs-7SB modifier. 
Table 12.14: Peaks identified from the ESI-MSof the 1st stripping solution post contact with the NG-CSSX solvent (irradiated in contact with SRS-15 for 50 MRad)

\begin{tabular}{clc}
\hline Peak m/z & \multicolumn{1}{c}{ Proposed species } & 0.5 MRad \\
\hline 361.02 & {$[(\mathrm{Cs}-7 \mathrm{SB}) \mathrm{Na}]^{+}$} & 361.14 \\
383.12 & {$[((\mathrm{Cs}-7 \mathrm{SB}-\mathrm{H}) \mathrm{Na}) \mathrm{Na}]^{+}$} & 383.12 \\
399.12 & {$[((\mathrm{Cs}-7 \mathrm{SB}-\mathrm{H}) \mathrm{Na}) \mathrm{K}]^{+}$} & 399.10 \\
406.42 & {$[(\mathrm{DCiTG}) \mathrm{H}]^{+}$} & 406.42 \\
415.03 & {$[((\mathrm{Cs}-7 \mathrm{SB}-\mathrm{H}) \mathrm{K}) \mathrm{K}]^{+}$} & 415.07 \\
743.24 & {$\left[((\mathrm{Cs}-7 \mathrm{SB}-\mathrm{H}) \mathrm{Na})_{2} \mathrm{Na}\right]^{+}$} & 743.25 \\
759.24 & {$\left[((\mathrm{Cs}-7 \mathrm{SB}-\mathrm{H}) \mathrm{Na})_{2} \mathrm{~K}\right]^{+}$} & 759.23 \\
775.24 & {$\left[((\mathrm{Cs}-7 \mathrm{SB}-\mathrm{H}) \mathrm{K})_{2} \mathrm{Na}\right]^{+}$} & 775.20 \\
875.54 & {$\left[\left(\left(\mathrm{MaxCalix}-\mathrm{C}_{10} \mathrm{H}_{21}\right) \mathrm{Na}\right) \mathrm{K}\right]^{+}$} & 875.39 \\
\hline
\end{tabular}

\subsection{THERMAL TREATMENT}

\subsubsection{General Observations}

Overall, the results presented below show that thermal treatment of the NG-CSSX solvent at $35{ }^{\circ} \mathrm{C}$ in contact with SRS-15 simulant, $25 \mathrm{mM} \mathrm{NaOH}$, and $10 \mathrm{mM} \mathrm{H}_{3} \mathrm{BO}_{3}$ will not adversely affect the ability of the solvent to perform within reference limits for 3 years of operation. As in the case of the radiationstability tests, the following ESS references (at $25^{\circ} \mathrm{C}$ ) are adopted as indicating a need for mitigation in that performance is far from design performance and a likely risk for meeting facility decontamination requirements: $D_{\mathrm{Cs}} \geq 40$ (extraction), $0.5 \leq D_{\mathrm{Cs}} \leq 10$ (scrub), $D_{\mathrm{Cs}} \leq 0.1$ (strip). In the operating process, elevated temperature is only expected in the strip section, which is heated to $33 \pm 3{ }^{\circ} \mathrm{C}^{30}$, but fortunately the solvent under stripping conditions is the least affected by thermal treatment. It was observed that, for the thermally treated NG-CSSX in contact with SRS-15 simulant and $25 \mathrm{mM} \mathrm{NaOH}$ scrub solution, the $D_{\mathrm{Cs}}$ values in ESS tests are not significantly affected until the solvent is exposed to more than 4 months of continuous contact at $35{ }^{\circ} \mathrm{C}$. Given that most of the solvent resides in a hold tank under ambient conditions, the thermal treatment is the equivalent of a 3 year duration at the MCU, even under conservative assumption that the solvent in the cooler extraction and scrubbing sections degrades as if the temperature were $35^{\circ} \mathrm{C}$. The test also does not take credit for any washing-out of degradation products that would occur under actual processing conditions with two wash stages.

\subsubsection{Control Solvent}

The control for the thermal-stability test was a sample of the initial batch of NG-CSSX solvent stored in the absence of any aqueous phase and maintained in darkness at ambient temperature. The $D_{\mathrm{Cs}}$ values for the $\mathrm{ES}_{2} \mathrm{~S}_{3}$ tests of the control solvent determined at the same times as for the thermally treated samples are given in Table 12.15. It was found that the $D_{\mathrm{Cs}}$ values for extraction and scrubbing vary little with time, 
averaging $60 \pm 5$ for extraction, $2.6 \pm 0.2$ for Scrub 1, and $1.1 \pm 0.1$ for Scrub 2. The $D_{\mathrm{Cs}}$ values for stripping, however, increased noticeably over time, though performance remained excellent overall. The changes suggest that unused solvent may have a shelf life of perhaps one year, at which time its condition will have changed and should be checked.

Table 12.15. $D_{\mathrm{Cs}}$ for ESS batch tests ${ }^{a}$ of untreated control solvent ${ }^{b}$ for thermal treatment

\begin{tabular}{lllllll}
\hline \multicolumn{1}{c}{ Stage } & \multicolumn{1}{c}{ 0 Months } & \multicolumn{1}{c}{ 1 Month } & \multicolumn{1}{c}{ 2 Months } & \multicolumn{1}{c}{ 3 Months } & 5 Months & 13 Months \\
\hline Extraction & $61.0(6)$ & $55(1)$ & $57.5(5)$ & $60.1(4)$ & $68(3)$ & $60(1)$ \\
Scrub 1 & $2.8(3)$ & $2.7(4)$ & $2.7(1)$ & $2.20(1)$ & $2.5(11)$ & $2.73(4)$ \\
Scrub 2 & $1.01(3)$ & $0.93(5)$ & $1.210(4)$ & $1.230(7)$ & $1.28(2)$ & $1.20(1)$ \\
Strip 1 & $1.3(1) \times 10^{-3}$ & $1.2(3) \times 10^{-3}$ & $2.6(1) \times 10^{-3}$ & $3.51(2) \times 10^{-3}$ & $5.2(2) \times 10^{-3}$ & $7.93(4) \times 10^{-3}$ \\
Strip 2 & $2.1(8) \times 10^{-4}$ & $4.3(5) \times 10^{-4}$ & $7(2) \times 10^{-4}$ & $6.4(1) \times 10^{-4}$ & $1.2(3) \times 10^{-3}$ & $2.17(3) \times 10^{-3}$ \\
Strip 3 & $1.5(2) \times 10^{-4}$ & $2(2) \times 10^{-4}$ & $5.83(3) \times 10^{-4}$ & $3.1(1) \times 10^{-4}$ & $5.21(7) \times 10^{-4}$ & $5.1(4) \times 10^{-4}$ \\
\hline
\end{tabular}

${ }^{a}$ The aqueous phases were SRS-15 waste simulant for extraction, $0.025 \mathrm{M} \mathrm{NaOH}$ for the scrub stages, and $0.01 \mathrm{M} \mathrm{H}_{3} \mathrm{BO}_{3}$ for the strip stages. Solvent composition is defined in Table 2.2. The O:A ratios were 1:4 for extraction and 3.75:1 for both scrubbing and stripping. Experiments were carried out at a constant temperature of $25 \pm 0.2^{\circ} \mathrm{C}$.

${ }^{b}$ Control solvent stored away from light at ambient temperature with no aqueous phase present.

\subsubsection{Thermal Treatment During Contact with SRS-15 Simulant}

ESS performance of NG-CSSX solvent proved sensitive to thermal treatment in the presence of SRS-15 simulant. The $D_{\mathrm{Cs}}$ values in an $\mathrm{ES}_{2} \mathrm{~S}_{3}$ test of the thermally treated NG-CSSX solvent whilst in contact with the waste simulant are given in Table 12.16. It was found that the $D_{\mathrm{Cs}}$ values for extraction (average $57 \pm 12)$ vary significantly compared with experimental precision $( \pm 22 \%$ versus $\pm 10 \%)$, perhaps as a symptom of minor solvent leakage (see Experimental Section) and greater random errors for measurements made over many weeks.

Table 12.16. $D_{\mathrm{Cs}}$ for ESS batch tests ${ }^{a}$ of NG-CSSX solvent in continuous contact with SRS-15 simulant at $35{ }^{\circ} \mathrm{C}$

\begin{tabular}{lllllll}
\hline \multicolumn{1}{c}{ Stage } & 0 Months & \multicolumn{1}{c}{ 1 Month } & \multicolumn{1}{c}{ 2 Months } & \multicolumn{1}{c}{ 3 Months } & \multicolumn{1}{c}{ 5 Months } & 13 Months \\
\hline Extraction & $61.0(6)$ & $74(5)$ & $40.4(2)$ & $52(1)$ & $52.70(6)$ & $57(1)$ \\
Scrub 1 & $2.8(3)$ & $4.2(3)$ & $3(1)$ & $1.89(6)$ & $2.93(3)$ & $2.84(4)$ \\
Scrub 2 & $1.01(3)$ & $1.89(5)$ & $1.4(5)$ & $1.25(2)$ & $1.78(2)$ & $1.82(4)$ \\
Strip 1 & $1.3(1) \times 10^{-3}$ & $1.12(2) \times 10^{-2}$ & $1.40(3) \times 10^{-2}$ & $3.62(6) \times 10^{-2}$ & $8.1(3) \times 10^{-2}$ & $4.12(3) \times 10^{-1}$ \\
Strip 2 & $2.1(8) \times 10^{-4}$ & $2.5(3) \times 10^{-3}$ & $3.9(9) \times 10^{-3}$ & $1.7(1) \times 10^{-2}$ & $1.6(2) \times 10^{-1}$ & $5.65(2) \times 10^{-1}$ \\
Strip 3 & $1.5(2) \times 10^{-4}$ & $1.0(3) \times 10^{-3}$ & $1.0(2) \times 10^{-3}$ & $3.5(5) \times 10^{-3}$ & $9.33(2) \times 10^{-2}$ & $5.98(6) \times 10^{-1}$ \\
\hline
\end{tabular}

${ }^{a}$ The aqueous phases were SRS- 15 waste simulant for extraction, $0.025 \mathrm{M} \mathrm{NaOH}$ for the scrub stages, and $0.01 \mathrm{M} \mathrm{H}_{3} \mathrm{BO}_{3}$ for the strip stages. Solvent composition is defined in Table 2.2. The O:A ratios were 1:4 for extraction and 3.75:1 for both scrubbing and stripping. ESS tests were carried out at a constant temperature of $25 \pm 0.2{ }^{\circ} \mathrm{C}$. 
However, no trend is observable with time, and the reference value of 40 is not crossed. The $D_{\mathrm{Cs}}$ values for scrubbing also exhibit some scatter without any apparent trend, with an average of $2.9 \pm 0.8$ for Scrub 1 and $1.5 \pm 0.3$ for Scrub 2. The $D_{\mathrm{Cs}}$ values for stripping, however increase quite significantly over time, crossing the reference limit of 0.1 after 4.6 months of continuous contact at $35{ }^{\circ} \mathrm{C}$.

\subsubsection{Thermal Treatment During Contact with $25 \mathrm{mM} \mathrm{NaOH}$}

Solvent treated in the present of scrub solution behaved almost identically to that of solvent treated in the presence of the SRS-15 simulant, indicating an important effect of alkalinity on solvent stability. The $D_{\mathrm{Cs}}$ values for $\mathrm{ES}_{2} \mathrm{~S}_{3}$ tests of the thermally treated NG-CSSX solvent whilst in contact with $25 \mathrm{mM} \mathrm{NaOH}$ are given in Table 12.17. It was found that the $D_{\mathrm{Cs}}$ values for extraction exhibit no apparent trend, averaging $65 \pm 5$. Scatter is also seen with the $D_{\mathrm{Cs}}$ scrubbing values with no apparent trend, averaging $4 \pm 1$ for Scrub 1 and $2 \pm 1$ for Scrub 2. As for solvent treated with the SRS-15 simulant, the $D_{\mathrm{Cs}}$ value for stripping again increases quite significantly over time, with crossover of the reference limit at 4 months.

Table 12.17. $D_{\mathrm{Cs}}$ for ESS batch tests ${ }^{a}$ of NG-CSSX solvent in continuous contact with $0.025 \mathrm{M} \mathrm{NaOH}$ at $35{ }^{\circ} \mathrm{C}$

\begin{tabular}{llclccc}
\hline \multicolumn{1}{c}{ Stage } & 0 Months & 1 Month & 2 Months & 3 Months & 5 Months & 13 Months \\
\hline Extraction & $61.0(6)$ & $70.9(5)$ & $61(4)$ & $65.1(2)$ & $67.5(3)$ & $60.8(4)$ \\
Scrub 1 & $2.8(3)$ & $5.68(4)$ & $4.0(16)$ & $2.4(1)$ & $3.7(2)$ & $3.04(3)$ \\
Scrub 2 & $1.01(3)$ & $4.4(15)$ & $1.25(3)$ & $1.27(1)$ & $1.63(0)$ & $1.56(2)$ \\
Strip 1 & $1.3(1) \times 10^{-3}$ & $2(1) \times 10^{-2}$ & $2.5(5) \times 10^{-2}$ & $4.91(7) \times 10^{-2}$ & $8.5(9) \times 10^{-2}$ & $2.90(9) \times 10^{-1}$ \\
Strip 2 & $2.1(8) \times 10^{-4}$ & $1.15(2) \times 10^{-3}$ & $9(1) \times 10^{-3}$ & $3.7(5) \times 10^{-2}$ & $2.0(2) \times 10^{-1}$ & $4.8(2) \times 10^{-1}$ \\
Strip 3 & $1.5(2) \times 10^{-4}$ & $9(6) \times 10^{-4}$ & $2.0(3) \times 10^{-3}$ & $8(2) \times 10^{-3}$ & $1.41(3) \times 10^{-1}$ & $5.17(6) \times 10^{-1}$ \\
\hline
\end{tabular}

${ }^{a}$ The aqueous phases were SRS-15 waste simulant for extraction, $0.025 \mathrm{M} \mathrm{NaOH}$ for the scrub stages, and $0.01 \mathrm{M}$ $\mathrm{H}_{3} \mathrm{BO}_{3}$ for the strip stages. Solvent composition is defined in Table 2.2. The O:A ratios were 1:4 for extraction and 3.75:1 for both scrubbing and stripping. Experiments were carried out at a constant temperature of $25 \pm 0.2^{\circ} \mathrm{C}$.

\subsubsection{Contact with $10 \mathrm{mM} \mathrm{H}_{3} \mathrm{BO}_{3}$ During Thermal Treatment}

By contrast to thermal treatment under alkaline conditions, treatment in the presence of the strip solution degraded performance much less. The $D_{\mathrm{Cs}}$ values for an $\mathrm{ES}_{2} \mathrm{~S}_{3}$ test of the thermally treated NG-CSSX solvent whilst in contact with $10 \mathrm{mM} \mathrm{H}_{3} \mathrm{BO}_{3}$ are given in Table 12.18. Again, it was found that the $D_{\mathrm{Cs}}$ values for extraction and scrubbing exhibit no trend, averaging $62 \pm 5$ for extraction, $4 \pm 1$ for Scrub 1 and $2 \pm 1$ for Scrub 2. The $D_{\mathrm{Cs}}$ values for stripping, however, increase over time in comparison with the control solvent, but the increase is an order of magnitude smaller than observed under alkaline conditions. This indicates that under neutral conditions the solvent is more resistant to thermal degradation than when contacted with alkaline solutions. 
Table 12.18. $D_{\mathrm{Cs}}$ for ESS batch tests ${ }^{a}$ of NG-CSSX solvent in continuous contact with $0.01 \mathrm{M} \mathrm{H}_{3} \mathrm{BO}_{3}$ at $35{ }^{\circ} \mathrm{C}$

\begin{tabular}{lccllll}
\hline \multicolumn{1}{c}{ Stage } & $\mathbf{0}$ & $\mathbf{1}$ Month & \multicolumn{1}{c}{ 2 Months } & 3 Months & 5 Months & 13 Months \\
\hline Extraction & $61.0(6)$ & $68(9)$ & $61(4)$ & $56(1)$ & $66.7(1)$ & $63(2)$ \\
Scrub 1 & $2.8(3)$ & $5.9(4)$ & $3.26(9)$ & $2.59(1)$ & $3.49(7)$ & $3.07(6)$ \\
Scrub 2 & $1.01(3)$ & $4(1)$ & $1.66(3)$ & $1.73(0)$ & $1.67(2)$ & $1.58(4)$ \\
Strip 1 & $1.3(1) \times 10^{-3}$ & $1.6(9) \times 10^{-2}$ & $7.9(2) \times 10^{-3}$ & $1.56(0) \times 10^{-2}$ & $2.1(3) \times 10^{-2}$ & $8.1(5) \times 10^{-2}$ \\
Strip 2 & $2.1(8) \times 10^{-4}$ & $3(4) \times 10^{-3}$ & $1.79(1) \times 10^{-3}$ & $4.8(5) \times 10^{-3}$ & $3.8(4) \times 10^{-3}$ & $1.72(3) \times 10^{-1}$ \\
Strip 3 & $1.5(2) \times 10^{-4}$ & $9(5) \times 10^{-4}$ & $7.4(7) \times 10^{-4}$ & $8.9(1) \times 10^{-4}$ & $2.3(6) \times 10^{-3}$ & $1.30(2) \times 10^{-1}$ \\
\hline
\end{tabular}

${ }^{a}$ The aqueous phases were SRS-15 waste simulant for extraction, $0.025 \mathrm{M} \mathrm{NaOH}$ for the scrub stages, and $0.01 \mathrm{M}$ $\mathrm{H}_{3} \mathrm{BO}_{3}$ for the strip stages. Solvent composition is defined in Table 2.2. The O:A ratios were 1:4 for extraction and 3.75:1 for both scrubbing and stripping. Experiments were carried out at a constant temperature of $25 \pm 0.2^{\circ} \mathrm{C}$.

\subsubsection{Electrospray Mass Spectrometry}

The composition of the thermally treated NG-CSSX was investigated using electrospray mass spectrometry. Significant changes in the composition of the mass spectra of the NG-CSSX solvent that had been contacted at $35{ }^{\circ} \mathrm{C}$ with sodium hydroxide $(25 \mathrm{mM})$ can be observed as the duration of thermal treatment is increased. As can be seen in Table 12.19 and Figs. 12.3, 12.4 and 12.5, the concentration of the DCiTG suppressor $\left([\mathrm{M}+\mathrm{H}]^{+}, \mathrm{m} / \mathrm{z}\left(\mathrm{C}_{13}\right)=406.4\right)$ is significantly reduced over time, potentially responsible for the significantly increase in the stripping $D_{C s}$ values (see Table 12.18). The increased presence of the amine at $\mathrm{m} / \mathrm{z} 200.1\left(\left[\mathrm{C}_{13} \mathrm{NH}_{3}\right]^{+}\right)$as well the amine bound to the extractant, $\left[\mathrm{Max}-\mathrm{R}_{1} \mathrm{NH}_{3}\right]^{+}$, at $\mathrm{m} / \mathrm{z} 1154.7$, suggests that base hydrolysis of the guanidine is occurring over time. A distinct increase in the peaks $[\mathrm{Max}-\mathrm{K}]^{+},[\mathrm{Max}-\mathrm{Na}]^{+},\left[\mathrm{Max}-\mathrm{NH}_{4}\right]^{+}$and $\left[\mathrm{Max}-\mathrm{R}_{1} \mathrm{NH}_{3}\right]^{+}$can be observed, which could partially explain the increased $D_{C s}$ values for scrubbing (see Table 12.17); suggesting that lipophilic anions are present in solution preventing the removal of the more weakly bound amines and alkaline metals.

Table 12.19. The major species observed in the ESI-MS of the NG-CSSX solvent after thermal treatment at $35^{\circ} \mathrm{C}$ whilst in contact with $\mathrm{NaOH}(25 \mathrm{mM})$ for $0,3,5$ and 13 months.

\begin{tabular}{|c|c|c|c|c|c|c|c|}
\hline $\begin{array}{c}\text { Time } \\
\text { (Months) }\end{array}$ & $\begin{array}{c}{\left[\mathrm{R}_{\mathbf{1}} \mathrm{NH}_{3}\right]^{+}} \\
(\mathbf{2 0 0 . 1})\end{array}$ & $\begin{array}{c}{[\mathrm{DCiTG}-} \\
\mathrm{H}]^{+}(\mathbf{4 0 6 . 4 )}\end{array}$ & $\begin{array}{c}\left.\text { Max- } \mathrm{NH}_{4}\right]^{+} \\
(972.6)\end{array}$ & $\begin{array}{c}{[\mathrm{Max}-\mathrm{Na}]^{+}} \\
(\mathbf{9 7 7 . 4 )}\end{array}$ & $\begin{array}{c}{[\mathrm{Max}-\mathrm{K}]^{+}} \\
(\mathbf{9 9 3 . 5})\end{array}$ & $\begin{array}{c}{[\mathrm{Max}-\mathrm{Cs}]^{+}} \\
(\mathbf{1 0 8 7 . 4 )}\end{array}$ & $\begin{array}{c}\text { [Max- } \\
\left.\mathrm{R}_{1} \mathrm{NH}_{3}\right]^{+} \\
(\mathbf{1 1 5 4 . 7})\end{array}$ \\
\hline 0 & $0 \%$ & $164 \%$ & $0 \%$ & $0 \%$ & $54 \%$ & $100 \%$ & $0 \%$ \\
\hline 3 & $4 \%$ & $79 \%$ & $6 \%$ & $8 \%$ & $67 \%$ & $100 \%$ & $3 \%$ \\
\hline 5 & $1 \%$ & $48 \%$ & $2 \%$ & $3 \%$ & $106 \%$ & $100 \%$ & $1 \%$ \\
\hline 13 & $123 \%$ & $6 \%$ & $231 \%$ & $139 \%$ & $570 \%$ & $100 \%$ & $102 \%$ \\
\hline
\end{tabular}




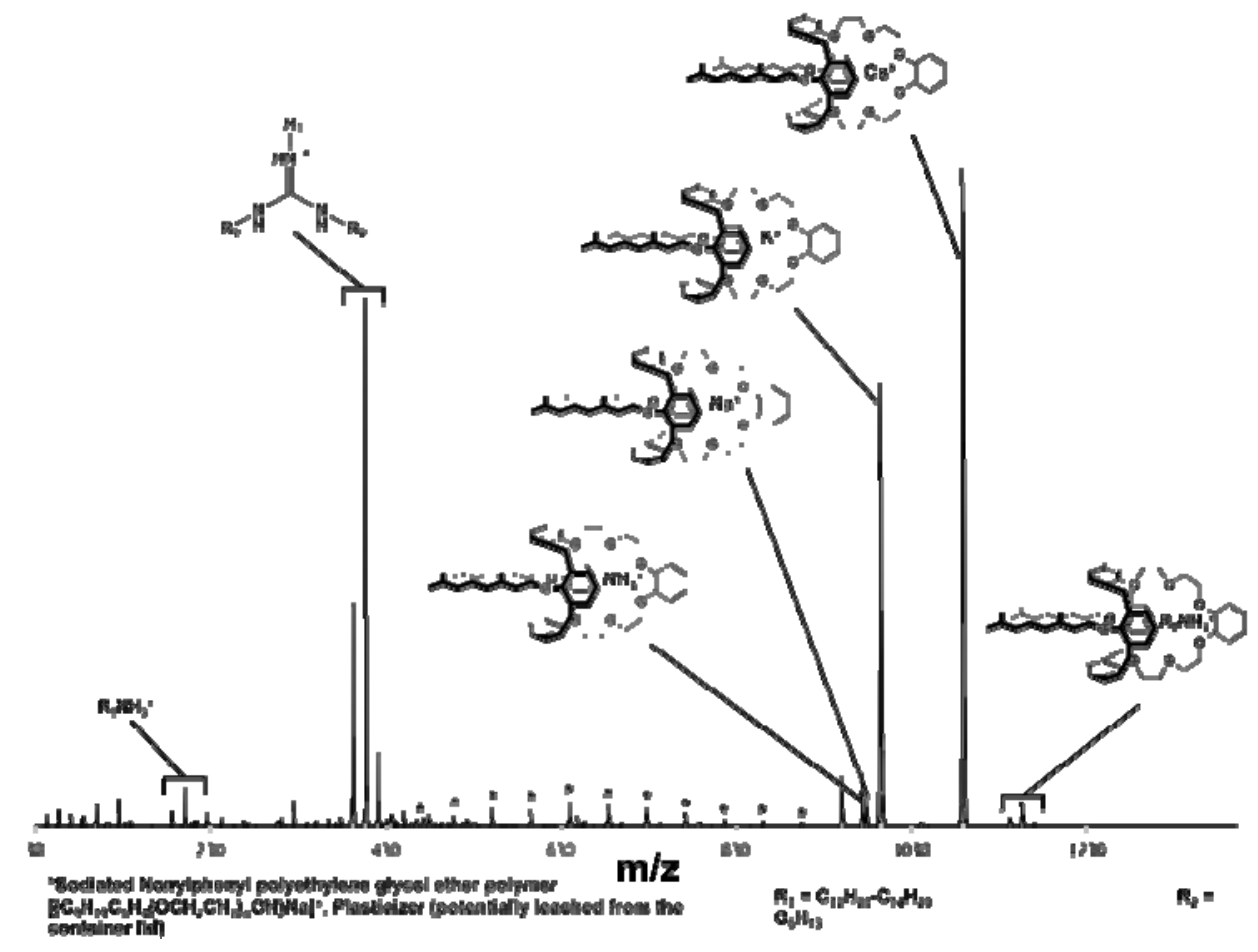

Fig. 12.3. ESI-MS of NG-CSSX solvent (1,000 fold dilution in MeCN) after 3 months thermal treatment whilst in contact with $\mathrm{NaOH}(25 \mathrm{mM})$.

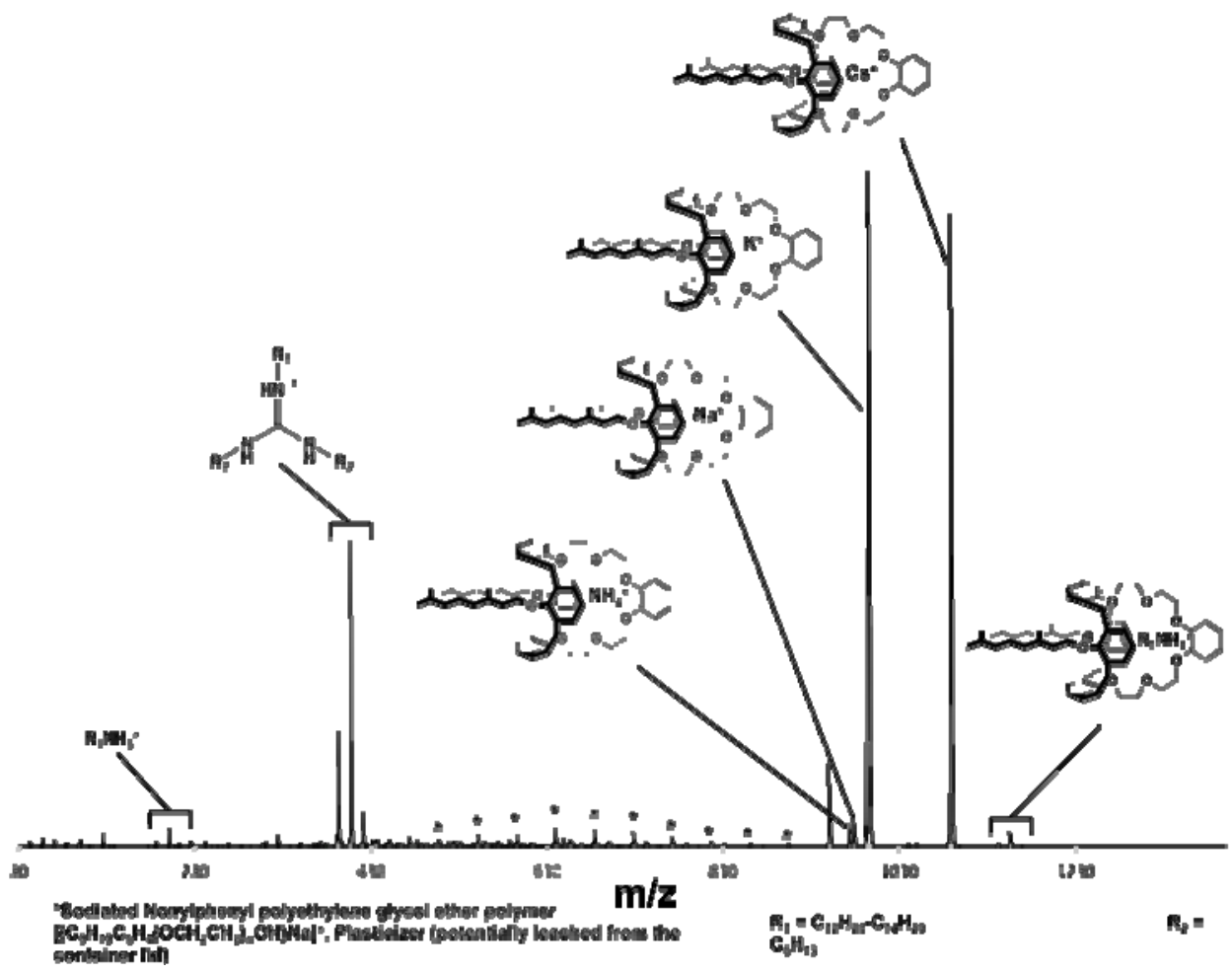

Fig. 12.4. ESI-MS of NG-CSSX solvent $(1,000$ fold dilution in $\mathrm{MeCN})$ after 5 months thermal treatment whilst in contact with $\mathrm{NaOH}(25 \mathrm{mM})$. 


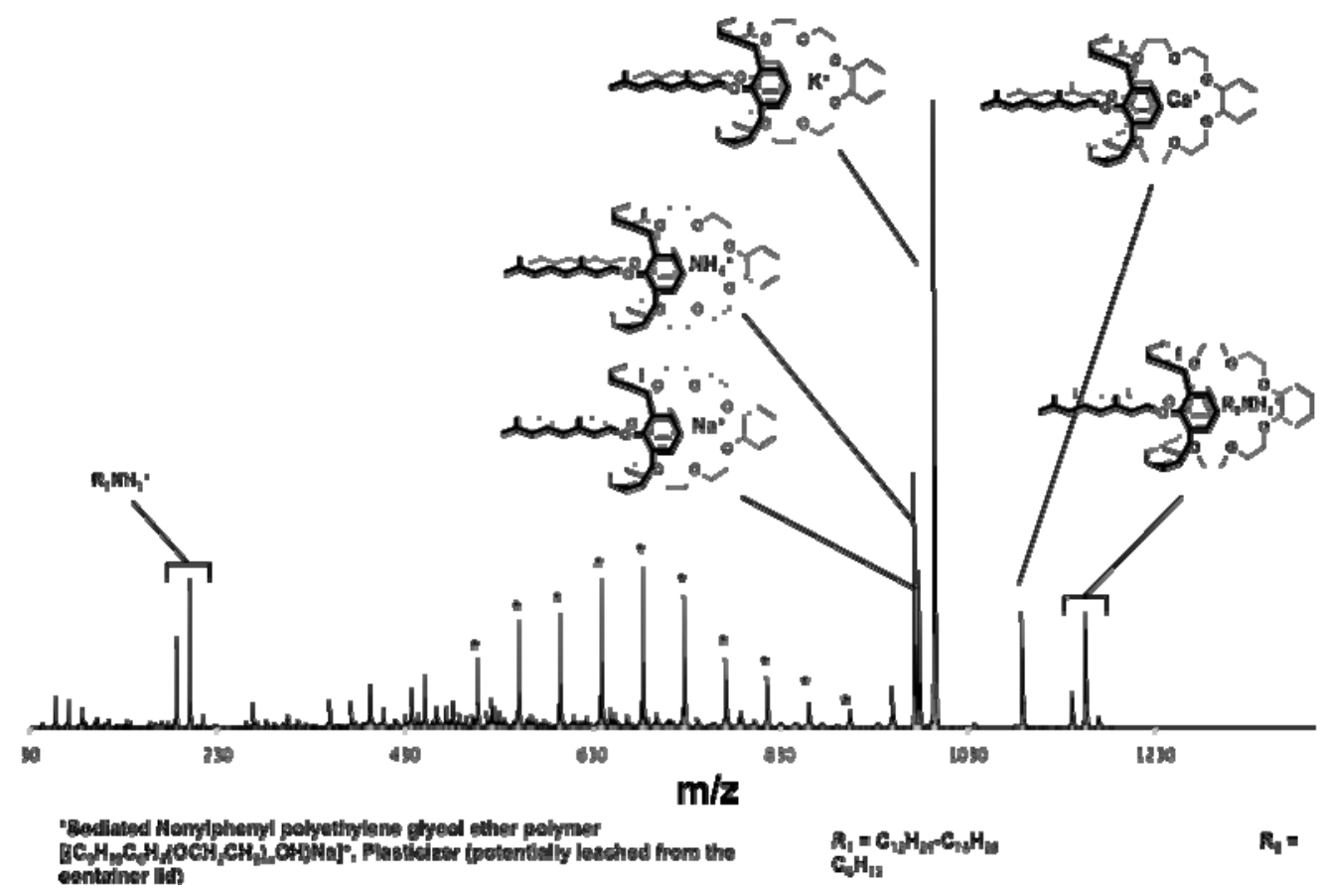

Fig. 12.5. ESI-MS of NG-CSSX solvent (1,000 fold dilution in MeCN) after 13 months thermal treatment whilst in contact with $\mathrm{NaOH}(25 \mathrm{mM})$.

After the initial mass spectrometry analyses the thermally treated solvent ( 5 and 13 month samples) were contacted a second time with fresh sodium hydroxide solution $(25 \mathrm{mM}$, O:A, 3.75:1) followed by 3 contacts with boric acid $(10 \mathrm{mM}, \mathrm{O}: \mathrm{A}, 3.75: 1)$ to complete an $\mathrm{ES}_{2} \mathrm{~S}_{3}$. A final contact with sodium hydroxide (25 mM, O:A, 3.75:1), which represented the solvent wash stage, was carried out and the washed solvents were again analyzed by ESI-MS. Figs. 12.6 and 12.7 detail the resulting data from ESIMS analyses after a 1000 fold dilution in acetonitrile (HPLC grade). At 5 months, after the stripping and wash stages, there is still visible $\left[\mathrm{Max}-\mathrm{NH}_{4}\right]^{+},[\mathrm{Max}-\mathrm{Na}]^{+},[\mathrm{Max}-\mathrm{K}]^{+}$, and $\left[\mathrm{Max}-\mathrm{R}_{1} \mathrm{NH}_{3}\right]^{+}$in the ESI mass spectrum. The $[\mathrm{Max}-\mathrm{Cs}]^{+}$appears to have essentially been removed and the $[\mathrm{DCiTG}-\mathrm{H}]^{+}$peak is still visible, despite the clear onset of DCiTG degradation, indicative by the presence of the Max-bound [Max$\left.\mathrm{RNH}_{3}\right]^{+}$, and the unbound $\left[\mathrm{RNH}_{3}\right]^{+}$(see Fig. 12.6). At 13 months, after the stripping and wash stages, the relative intensities of the $\left[\mathrm{Max}-\mathrm{NH}_{4}\right]^{+},[\mathrm{Max}-\mathrm{Na}]^{+},[\mathrm{Max}-\mathrm{K}]^{+}$, and $\left[\mathrm{Max}-\mathrm{R}_{1} \mathrm{NH}_{3}\right]^{+}$peaks have not changed dramatically. The major differences are the presence of the $[\mathrm{Max}-\mathrm{Cs}]^{+}$peak, indicating incomplete removal of cesium from the solvent, and the lack of the $[\mathrm{DCiTG}-\mathrm{H}]^{+}$peak, suggesting complete degradation of the DCiTG guanidine suppressor to the primary amine responsible for the $\left[\mathrm{RNH}_{3}\right]^{+}$and $\left[\mathrm{Max}-\mathrm{RNH}_{3}\right]^{+}$peaks (see Fig. 12.7). 


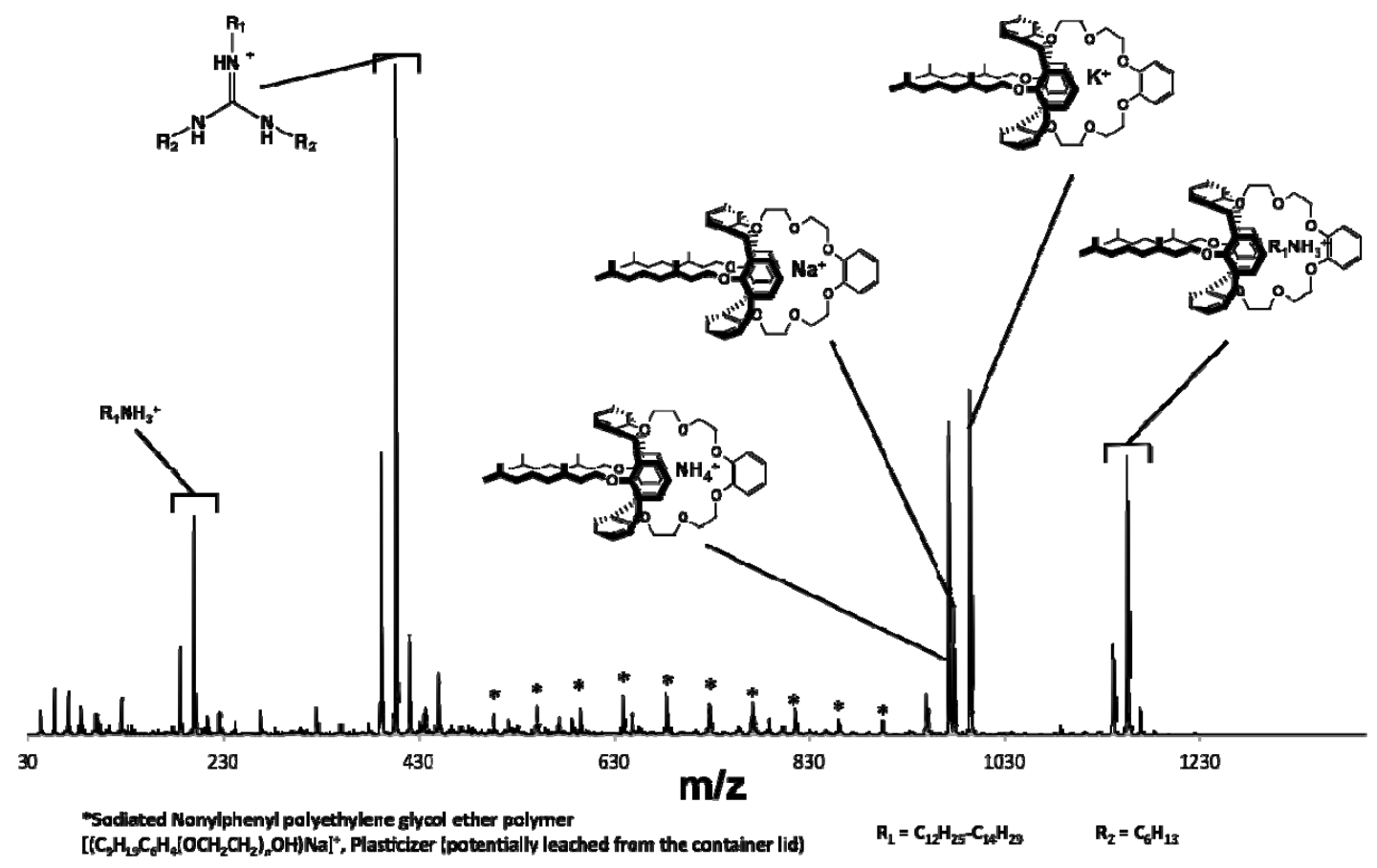

Fig. 12.6. ESI-MS of NG-CSSX solvent (1,000 fold dilution in MeCN) after 5 months thermal treatment whilst in contact with $\mathrm{NaOH}(25 \mathrm{mM})$ followed by consecutive contacts at an O:A of 3.75:1 with $\mathrm{NaOH}$ (25 mM), $2 \times \mathrm{H}_{3} \mathrm{BO}_{3}(10 \mathrm{mM})$, and $\mathrm{NaOH}(25 \mathrm{mM})$. 


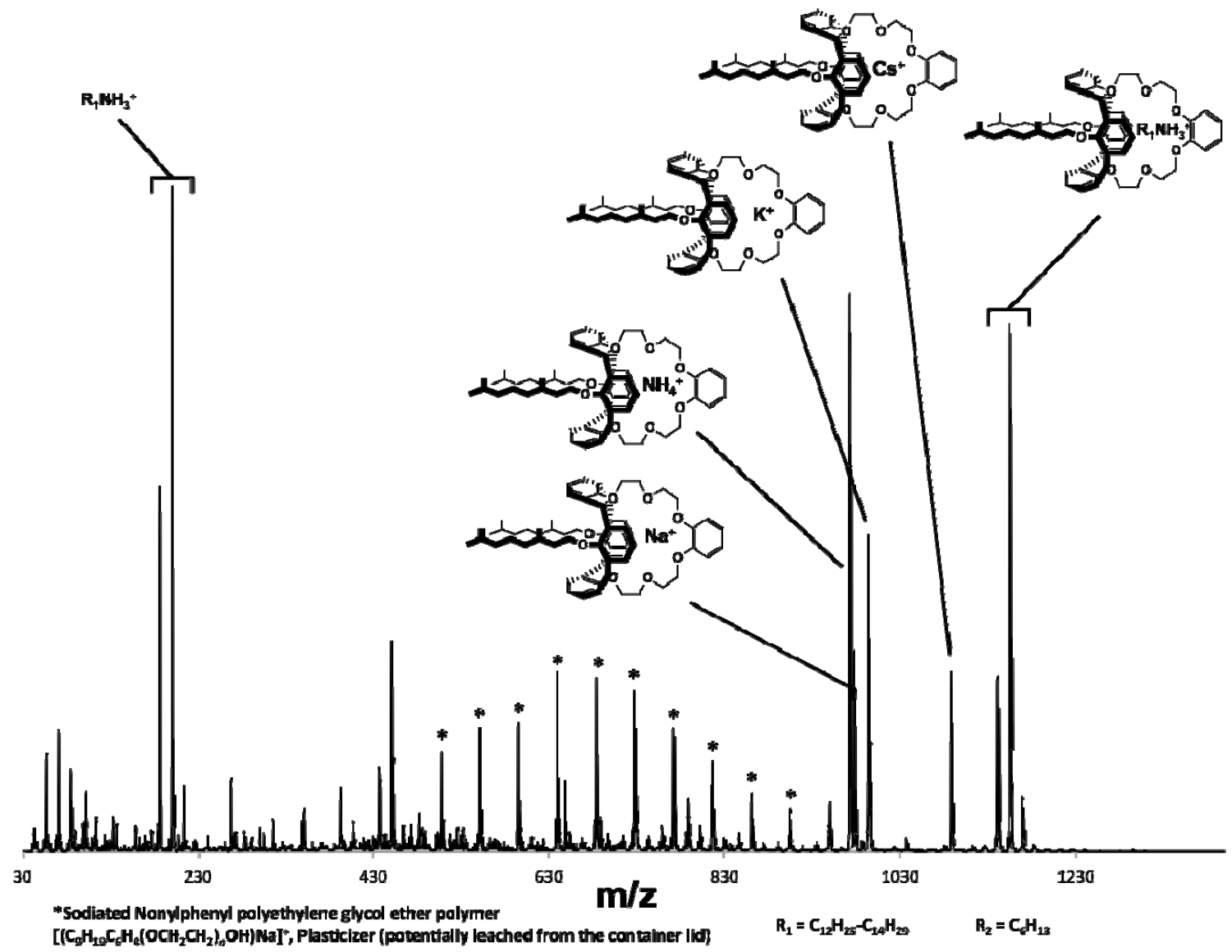

Fig. 12.7. ESI-MS of NG-CSSX solvent (1,000 fold dilution in MeCN) after 13 months thermal treatment whilst in contact with $\mathrm{NaOH}(25 \mathrm{mM})$ followed by consecutive contacts at an O:A of 3.75:1 with $\mathrm{NaOH}$ $(25 \mathrm{mM}), 2 \times \mathrm{H}_{3} \mathrm{BO}_{3}(10 \mathrm{mM})$, and $\mathrm{NaOH}(25 \mathrm{mM})$.

\subsubsection{Sodium Capacity of Thermally Treated Solvent}

Thermal treatment also resulted in an increase in the sodium capacity of the NG-CSSX solvent. The sodium concentrations, $[\mathrm{Na}]^{+}$, in $\mathrm{mM}$ for each of the thermally treated solvents after contact with $\mathrm{NaOH}$ (0.5 M, O:A 1:1) are given in Table 12.20. Over the 13-month period of thermal treatment, a 2-3 fold increase in the levels of sodium extracted by solvent occurs. This increase in sodium loading capability together with the $\mathrm{ES}_{2} \mathrm{~S}_{3}$ data, again, suggests an increase in the presence of lipophilic anions in the thermally treated solvents, facilitating the transferal of sodium to the solvent.

Table 12.20. Sodium loading in the solvents thermally treated with SRS-15, $\mathrm{NaOH}(25 \mathrm{mM})$, and $\mathrm{H}_{3} \mathrm{BO}_{3}$ $(10 \mathrm{mM})$ determined using $\mathrm{Na22}$ by contacting the solvent with $\mathrm{NaOH}(0.5 \mathrm{M})$ at an O:A ratio of 1:1 


\begin{tabular}{rccc}
\hline & ([Na] $^{+}$in solvent, $\left.\mathbf{~ m M}\right)$ & ([Na] in solvent, $\mathbf{m M})$ & ([Na] in solvent, $\mathbf{m M})$ \\
\hline 0 & $0.58(1)$ & $0.48(1)$ & $0.58(4)$ \\
3 & $0.90(2)$ & $0.61(2)$ & $0.52(1)$ \\
5 & $1.5(1)$ & $0.97(4)$ & $0.82(1)$ \\
13 & $1.77(6)$ & $1.34(6)$ & $1.09(2)$ \\
\hline
\end{tabular}

\subsubsection{Impact of Thermal Treatment on Process Performance}

Overall, the data presented above support a solvent lifetime of three years under process thermal stresses. The conditions used to test the thermal stability of the NG-CSSX solvent were chosen to be conservative with respect to the conditions and practice at the MCU (and also expected for SWPF). In this study, the solvent is in constant contact with the same aqueous phase, in contrast to the MCU where at any one time only $12 \%$ of the total solvent is in contact with an aqueous phase (see Table 12.21) and where the solvent is continuously washed by process solutions in sequence. In comparison with the irradiation tests, where scrubbing $D_{\mathrm{Cs}}$ values were the first to reach the reference limit, it is the stripping $D_{\mathrm{Cs}}$ values that first exceed reference limit in the thermally stressed solvent. The contacts that have shown the most increase in stripping $D_{\mathrm{Cs}}$ values, the extraction and stripping stages, are also held at significantly lower temperatures $\left(10^{\circ} \mathrm{C}\right.$ less) at the $\mathrm{MCU}$, and thus one would therefore expect significant reductions in solvent degradation kinetics. Even so, if one assumes conservatively that degradation in extraction and scrubbing would occur at the same rate as found for $35{ }^{\circ} \mathrm{C}$ in the present tests, then after 4 months the stripping would still function within limits, equivalent to a three year lifetime for the bulk solvent inventory at the MCU.

It can be shown by using the $D_{\mathrm{Cs}}$ values observed for each of the ESS stages and the average time that the NG-CSSX solvent spends in each stage (Table 12.22) that the performance of the NG-CSSX solvent remains within reference limits for the entire duration (13 months) of the experiment. Real-time process $D_{\mathrm{Cs}}$ values measured for the control solvent were used to represent the solvent hold tank stage, and the scrub stage. These $D_{\mathrm{Cs}}$ values were also used for the wash stage, given the similarity in the aqueous feeds (10 mM NaOH cf. $25 \mathrm{mM} \mathrm{NaOH})$.

Table 12.21. Assumptions for solvent volumes ${ }^{41}$ and temperatures ${ }^{30,41}$ for estimation of thermal degradation

\begin{tabular}{cccccc}
\hline Stage & $\begin{array}{c}\text { Solvent hold } \\
\text { tank }\end{array}$ & Extraction & Scrubbing & Stripping & Washing \\
\hline Solvent Volume & $\sim 150 \mathrm{gal}$ & $11 \mathrm{gal}$ & $1.5 \mathrm{gal}$ & $7 \mathrm{gal}$ & $1.5 \mathrm{gal}$ \\
\hline
\end{tabular}




\begin{tabular}{cccccc}
\hline$\%$ Solvent & $88 \%$ & $6 \%$ & $1 \%$ & $4 \%$ & $1 \%$ \\
Stage temperature & $33 \pm 3{ }^{\circ} \mathrm{C}$ & $23 \pm 3{ }^{\circ} \mathrm{C}$ & $26{ }^{\circ} \mathrm{C} \max$ & $33 \pm 3{ }^{\circ} \mathrm{C}$ & $33 \pm 3{ }^{\circ} \mathrm{C}$ \\
\hline
\end{tabular}

Table 12.22. Estimated ESS $D_{\mathrm{Cs}}$ for NG-CSSX solvent inventory over time at $35{ }^{\circ} \mathrm{C}^{a}$

\begin{tabular}{lllllll}
\hline \multicolumn{1}{c}{ Stage } & \multicolumn{1}{c}{ 0 } & 1 Month & 2 Months & 3 Months & 5 Months & 13 Months \\
\hline Extraction & 61 & 56.8 & 56.7 & 59.6 & 66.9 & 60.0 \\
Scrub 1 & 2.78 & 2.95 & 2.83 & 2.20 & 2.60 & 2.76 \\
Scrub 2 & 1.01 & 1.19 & 1.24 & 1.26 & 1.33 & 1.26 \\
Strip 1 & $1.30 \times 10^{-3}$ & $2.77 \times 10^{-3}$ & $3.90 \times 10^{-3}$ & $6.87 \times 10^{-3}$ & $1.20 \times 10^{-2}$ & $4.07 \times 10^{-2}$ \\
Strip 2 & $2.06 \times 10^{-4}$ & $6.83 \times 10^{-4}$ & $1.11 \times 10^{-3}$ & $2.53 \times 10^{-3}$ & $1.47 \times 10^{-2}$ & $5.23 \times 10^{-2}$ \\
Strip 3 & $1.49 \times 10^{-4}$ & $3.27 \times 10^{-4}$ & $6.41 \times 10^{-4}$ & $6.67 \times 10^{-4}$ & $8.97 \times 10^{-3}$ & $5.19 \times 10^{-2}$ \\
\hline
\end{tabular}

${ }^{a}$ Based on the percentage of solvent in each stage at any one time given in Table 12.21.

\subsection{CONCLUSIONS}

Although effects of chemical degradation are clearly evident in the coloration and in the ESS testing of solvent subjected to thermal and radiolytic stresses, test results show that NG-CSSX solvent would perform within acceptable limits for up to 3 years of process operation. This conservative conclusion does not take credit for the continuous washing-out of deleterious degradation products into the aqueous effluent streams under actual process conditions. Acceptable performance limits are adopted as $D_{\mathrm{Cs}} \geq 40$ (extraction), $0.5 \leq D_{\mathrm{Cs}} \leq 10$ (scrub), $D_{\mathrm{Cs}} \leq 0.1$ (strip). Upon correction for the fact that only a small fraction of the solvent inventory actually undergoes degradative stress at any given time, test results imply acceptable performance for up to 3 years based on thermal stability only. Effects of radiolysis are even less, with performance expected to remain within limits for 13 years. On the other hand, there remain questions that should be addressed (see below).

In both the thermal and radiolytic degradation studies, the most severe degradation occurred in the presence of the SRS-15 simulant, though the thermal degradation in the presence of $25 \mathrm{mM} \mathrm{NaOH}$ was equally severe. The observed increase in $D_{\mathrm{Cs}}$ values for stripping in both studies indicates that possible impurities formed upon degradation of the solvent will eventually impair stripping. Scrubbing $D_{\mathrm{Cs}}$ values increased in response to radiation, actually reaching the reference limit before the stripping $D_{\mathrm{Cs}}$ values. In contrast, the scrubbing $D_{\mathrm{Cs}}$ values were not much affected by thermal treatment. If an increase in scrubbing $D_{\mathrm{Cs}}$ values is assumed to reflect the presence of anionic impurities, then it would follow that thermal treatment does not produce anionic degradation products as fast as the radiation treatment. Moreover, the worsening of stripping on thermal treatment must then imply that the chief effect must be the degradation of the guanidine. Extraction $D_{\mathrm{Cs}}$ values were relatively stable under both thermal and radiation treatment and never fell below the reference value of 40 . 


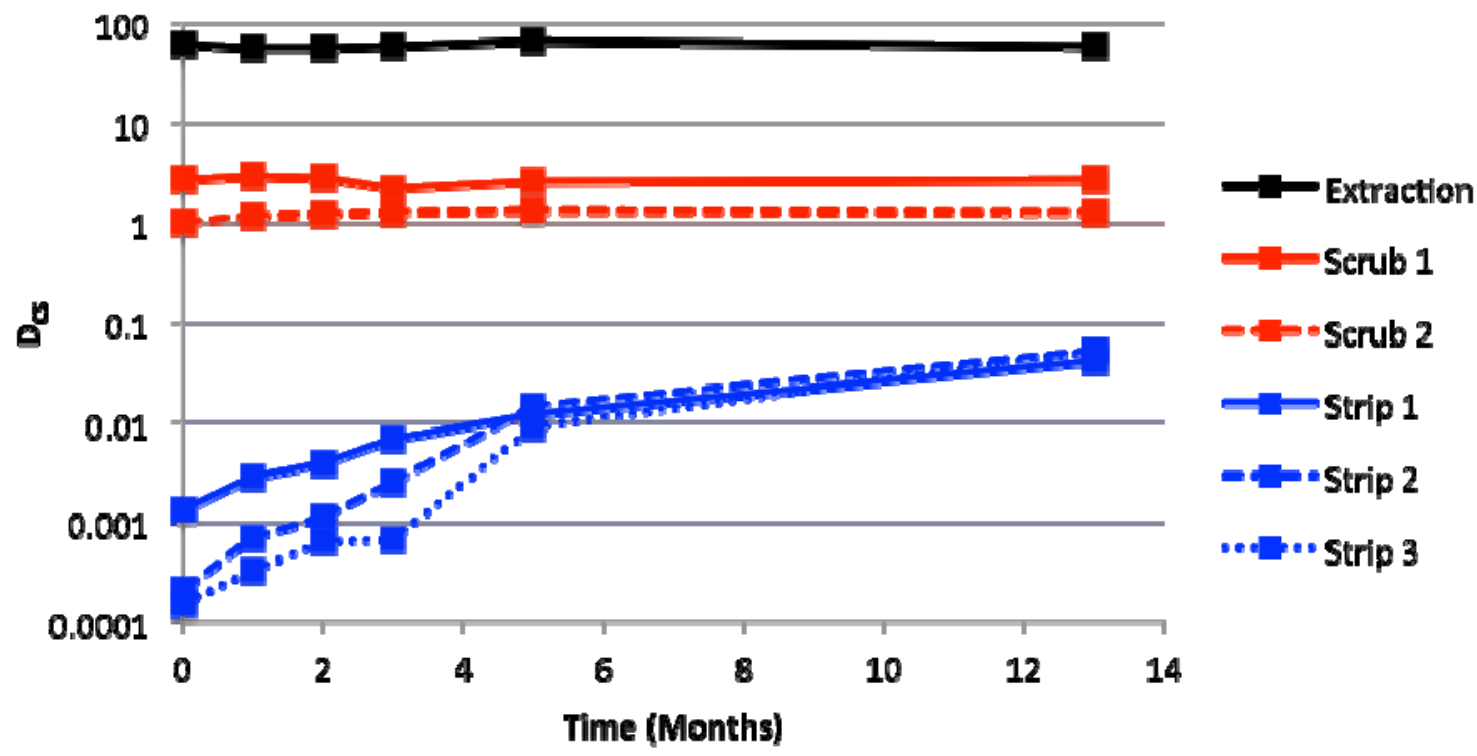

Fig. 12.8. Calculated $D_{\mathrm{Cs}}$ for NG-CSSX solvent inventory plotted versus time. Solvent fraction in the contactors is assumed to be at $35^{\circ} \mathrm{C}$. The $\mathrm{Y}$ axis is shown on a log scale. Data points are taken from Table 12.17. Shaded areas represent reference limits.

It was shown that, when in contact with $10 \mathrm{mM} \mathrm{H}_{3} \mathrm{BO}_{3}$ solution, the NG-CSSX solvent shows the most radiolytic and thermal stability. Alkaline conditions therefore appear to be the most severe. Interestingly, the pristine solvent used as the reference in the thermal treatment study appeared to show mild aging effects and possible degradation over time, even when uncontacted and untreated. A possible reason for this is reaction of the suppressor DCiTG with other solvent components. The guanidine suppressor is strongly basic (with a possible $\mathrm{p} K_{\mathrm{a}}$ in the range $12-13.5^{42}$ ) such that, when present in the deprotonated form, it may deprotonate or attack other solvent components, leading to further reactions.

Although the identities of certain major degradation products have been confirmed via ESI-MS and GC, their fates, and individual effects are currently unknown. The fate of these degradation products needs to be determined, if necessary by measuring their partitioning between the solvent and the process aqueous solutions, along with their potential to build up in the solvent. On this basis, defining a suitable solventwash solution also needs to be considered with an aim to demonstrate restoration of the degraded solvent.

Other factors also need to be clarified. The present positive interpretation of the results for a 3-year solvent lifetime only takes into account independent degradation processes, and increased levels of impurities from simultaneous thermal and radiolytic processes may influence solvent performance in an unknown manner. In addition, the ESS testing does not reflect a countercurrent configuration in which 
impurities can become effectively trapped in a reflux loop, as is possible between the extraction and scrub sections. Quite possibly, 4-sec-butylphenol, the main decomposition product of the Cs-7SB modifier, could exhibit such behavior. The stability of NG-CSSX solvent with regards to $\mathrm{HNO}_{3}$ should also be investigated. It was previously reported ${ }^{21}$ that LIX $^{\circledR} 79$ guanidine suppressor performed poorly following a prewash of the solvent with dilute nitric acid, raising the question of stability of the guanidine in the presence of nitric acid, a favorite solution for cleaning contactors.

Although a 1-year solvent lifetime was previously set as a performance standard ${ }^{7}$, in fact, multiyear service is desired in view of the great expense of procuring the solvent and likely comparable cost of its disposal. Perhaps even more costly would be the down-time that would be borne upon replacing the solvent. Given that no standard exists for deciding on solvent replacement, even for BOBCalixC6 CSSX, costs related to schedule delay as process problems are diagnosed, decisions made, and actions taken could easily exceed $\$ 10 \mathrm{M}$. The long-term stable performance of the NG-CSSX solvent is thus a critical matter of cost and schedule, and an improved knowledge of the chemistry involved in degradation and a better understanding of the identity and partitioning of the degradation products formed would provide the applicable basis for establishing a solvent-wash chemistry, setting process operational parameters, developing the needed process monitoring, and facilitating inevitable troubleshooting. As a strong precedent, the successful operation of the MCU since April $2008^{31}$ without a solvent replacement is in part attributable to the enabling research that was directed at these questions of solvent stability and the fate of impurities. 


\section{SUMMARY OF ACCOMPLISHMENTS}

\subsection{DOCUMENTATION IN REPORTS, PUBLICATIONS, AND PRESENTATIONS}

This report encompasses work performed by ORNL and its partners concerning the development of the NG-CSSX technology during the 2010 through 2012 period. A complete listing is provided at the end of this report, together with a list of publications and presentations. For expediency in the climate of an accelerated development and testing program, the reports have been reviewed (internally at ORNL in all cases and externally by project participants at ANL and SRNL in the majority of cases), cleared for release, and issued as letter reports to a limited distribution of DOE managers, national laboratory scientists and engineers, contractors, and site personnel having an interest in tank-waste cleanup. However, the reports are full technical reports, complete with applicable details regarding their specific topic. It is planned to convert the content of the letter reports, and also some results not yet documented, to open literature that will remain permanently retrievable. Accordingly, it is planned to publish the results in a combination of journal articles, conference proceedings, and publicly available ORNL-TM type reports. Much of the content of the letter reports is of a nature and quality sufficient for publication in journal articles, which offer an additional layer of quality assurance and validation through external peer review.

At the present time, three peer-reviewed publications and twenty-one reports (including this one) have been issued. Two of the publications were in the form of peer-reviewed conference proceedings articles and one was a journal article. Six presentations have been given, four at national meetings and two at an international forum.

\subsection{ACCOMPLISHMENTS}

\subsubsection{Solvent Chemistry Development}

\subsubsection{Optimized solvent composition}

The optimized NG-CSSX solvent composition chosen for flowsheet testing and demonstration for MCU deployment is shown in Table 13.1 (also see Chapter 3). The NG-CSSX solvent composition consists of 0.05 M MaxCalix cesium extractant, 0.5 M Cs-7SB modifier, and 0.003 DCiTG guanidine in Isopar $^{\circledR} \mathrm{L}$ diluent. It will meet the target DF of 40,000 in the MCU equipment configuration, assuming at least a $90 \%$ stage efficiency ${ }^{4}$ (also see Chapter 3 ). 
Table 13.1. NG-CSSX solvent components

\begin{tabular}{|c|c|c|c|}
\hline Component & Function & Chemical Name & Structure \\
\hline $\begin{array}{c}\text { MaxCalix } \\
0.05 \mathrm{M}\end{array}$ & Extractant & $\begin{array}{l}\text { 1,3-alt-25,27-Bis(3,7- } \\
\text { dimethyloctyl-1-oxy) } \\
\text { calix[4]arene- } \\
\text { benzocrown-6 }\end{array}$ & \\
\hline $\begin{array}{l}\text { Cs-7SB } \\
0.5 \mathrm{M}\end{array}$ & Modifier & $\begin{array}{l}\text { 1-(2,2,3,3- } \\
\text { Tetrafluoropropoxy)-3- } \\
\text { (4-sec-butylphenoxy)-2- } \\
\text { propanol }\end{array}$ & \\
\hline $\begin{array}{l}\text { DCiTG } \\
0.003 \mathrm{M}\end{array}$ & Suppressor & $\begin{array}{l}N, N \text {-Dicyclohexyl- } N " \text { - } \\
\text { isotridecylguanidine }\end{array}$ & \\
\hline Isopar L & Diluent & $\begin{array}{l}\mathrm{C}_{12} \text {-isoparaffinic } \\
\text { hydrocarbon }\end{array}$ & \\
\hline
\end{tabular}

The NG-CSSX solvent has undergone considerable evolution and even still is evolving. A monocrown calixarene called MaxCalix (Table 13.1) (see Chapter 3) has been selected as the best choice as the cesium extractant in the NG-CSSX solvent. With its high solubility, resistance to third-phase formation, and cesium extraction strength comparable to BOBCalixC6, it has simultaneously allowed higher DF and higher throughputs to be obtained. Higher DFs can be obtained because of the high extraction $D_{\mathrm{Cs}}$ values that result from use of much higher calixarene concentrations than was possible with BOBCalixC6 in the baseline BOBCalixC6 CSSX process. An earlier choice of $0.020 \mathrm{M}$ as the concentration for MaxCalix (see Chapter 3) was found in flowsheet calculations not to be high enough to guarantee a cesium DF of $\geq 40,000$ in the MCU configuration (see Chapter 3). MaxCalix supplanted the earlier leading calixarene candidate, called BEHBCalix, which readily formed a third phase at temperatures approaching preferred process temperatures and was therefore deemed not suitable. The Cs-7SB modifier is necessary for solubilizing all other solvent components and extracted species and for increasing cesium extraction strength. It functions well, but because it contributes to solvent viscosity and density, it was beneficial for contactor hydraulics to reduce its concentration in NG-CSSX to $0.5 \mathrm{M}$ from the $0.75 \mathrm{M}$ level used in BOBCalixC6 CSSX. The guanidine DCiTG enables stripping with boric acid, replacing the tri-n-octylamine (TOA) used as the suppressor in BOBCalixC6 CSSX. A stronger base is needed as the suppressor in NG-CSSX, because of the higher $\mathrm{pH}$ of the boric acid stripping conditions. It should be noted that recent experiments ${ }^{6}$ have indicated that it would be beneficial to replace DCiTG with a more lipophilic alternative suppressor, as the data show that DCiTG partitions somewhat to the aqueous boric acid strip solution. 


\subsubsection{Third-phase formation temperatures for NG-CSSX solvent}

Like the BOBCalixC6 CSSX solvent, the NG-CSSX solvent forms a third phase in contact with waste simulant as the temperature is decreased below a critical threshold. This temperature increases with potassium content of the waste and was shown to be $12{ }^{\circ} \mathrm{C}$ for NG-CSSX solvent in contact with average SRS waste at the bounding potassium concentration (see Chapters 3 and 8). Data for an earlier NG-CSSX composition with 0.02 M MaxCalix have also been reported (see Chapter 3). Effects of the concentrations of modifier and MaxCalix as well as extensions to BOBCalixC6 and BEHBCalix were also determined (unreported results).

\subsubsection{Solvent specifications}

Purity specifications for MaxCalix and for DCiTG have been issued (see Chapter 6) which allows the procurement of these two solvent components.

\subsubsection{Preparation of DCiTG suppressor}

A preparative method for the DCiTG suppressor was developed (see Chapter 4). The guanidine suppressors that have been under investigation include the active agent of the LIX 79 (DCiTG L), a commercially available reagent supplied by Cognis; DCiTG-E, a reagent synthesized at ORNL; and DCiTG-M1 and DCiTG-M2, two batches of suppressor synthesized using different conditions by Marshallton Research Laboratories. At the outset of the development of NG-CSSX, DCiTG L (LIX 79 guanidine) was the only available form of DCiTG. However, Cognis could provide only gram quantities out of existing stock (which did, however, support considerable progress in NG-CSSX development) and was unable to fill orders for less than $10 \mathrm{t}$. Quantities needed for MCU or SWPF solvent inventory are on the order of only $1 \mathrm{~kg}$, and thus, a new source of DCiTG was sought. At ORNL, a preparation was developed based in part on the Cognis patent (now expired), but employing EXXAL 13 as the starting alcohol. Marshallton Research Laboratories subsequently made available their own variant of DCiTG, employing the isotridecyl alcohol feedstock Jarcol $^{\mathrm{TM}}$ I-13. Whereas EXXAL 13 has a distribution of molecular weights around a carbon number of 13, Jarcol I-13 has only 13-carbon branched chains, though still with many isomers.

\subsubsection{Analytical method for DCiTG suppressor}

A method for the analysis of DCiTG by GC and its detection by mass spectrometry and FID was developed (see Chapter 5). The analytical method applies both to the analysis of DCiTG in synthetic samples and in the NG-CSSX solvent mixture. 


\subsubsection{Characterization of DCiTG suppressor}

The performance of four variants of the DCiTG suppressor was shown to be almost equivalent, as characterized by ESS tests and by measurement of the partitioning to the aqueous boric acid strip solution ${ }^{6}$. Since the starting isotridecanol used in the preparation of DCiTG is a mixture of branchedchain aliphatic alcohols varying in composition with manufacturer, the resulting DCiTG itself is a mixture. Thus, it is necessary to address how the solvent performance will be affected by the different preparations of the DCiTG solvent component. ESI-MS analysis was used to identify the major components of four DCiTG variants from three sources. In batch ESS tests, it was shown that the source of and, to a certain extent, the purity of the guanidine has little effect on the cesium distribution behavior of the NG-CSSX solvent for three simulants of tank salt waste. The simulants included average SRS salt waste simulant (SRS-15), SRS salt waste simulant at the bounding potassium concentration (SRS-45), and Hanford tank 241-AP-108 simulant with a moderately high potassium concentration. It was also shown that the concentration of the suppressor (nominally $3 \times$ $10^{-3} \mathrm{M}$ ) could drop to as low as $2.5 \times 10^{-4} \mathrm{M}$ before stripping performance is significantly impaired. While the potassium concentration in the feed apparently does not affect the function of the guanidine suppressor in stripping, the addition of the surfactant SDS to the SRS-15 simulant has a strong effect, revealing a wide range of abilities among the DCiTG candidates to suppress the effect of surfactant anions on stripping. The significant partitioning of all of the tested DCiTG suppressors to the strip solution as measured by GC is, however, a concern, though one with a ready solution, either through added process monitoring or replacement with an alternative guanidine. Accordingly, future work is recommended to develop a more lipophilic guanidine analog that is less aqueous soluble and also that lends itself to easier analysis.

\subsubsection{Preparation of guanidine alternatives to DCiTG}

Several new guanidine suppressors were prepared and characterized as alternatives to DCiTG (unreported results). Preparative methods were identified for various DCiTG analogs, primarily with replacement alkyl groups for the isotridecyl group in DCiTG. Cesium distribution measurements in batch ESS tests showed that the performance of the different suppressors does not vary strongly with guanidine structure. Unfortunately, none of the candidates employed larger alkyl groups than isotridecyl and thus are not sufficiently lipophilic to solve the problem of the partitioning loss of DCiTG to the aqueous boric acid strip solution. The synthetic methodology gained, however, will enable the rapid preparation of DCiTG analogs with higher lipophilicity more appropriate for process 
use.

\subsubsection{Solvent stability to process thermal/chemical and radiation stresses}

From solvent-degradation test results, it may be projected that the performance of the NG-CSSX solvent will remain within acceptable limits for 3 years under conditions applicable to the MCU or SWPF (see Chapters 11 and 12). Solvent samples were subjected to thermal treatment for 5 months at $35{ }^{\circ} \mathrm{C}$ in the presence of SRS-15 salt waste simulant, $0.025 \mathrm{M} \mathrm{NaOH}$ scrub solution, and $0.010 \mathrm{M}$ boric acid strip solution. In radiation-degradation tests, solvent samples were subjected to external gamma irradiation in a ${ }^{60} \mathrm{Co}$ source for up to $50 \mathrm{kGy}$ absorbed dose in the presence of SRS-15 salt waste simulant, $0.025 \mathrm{M} \mathrm{NaOH}$ scrub solution, and $0.010 \mathrm{M}$ boric acid strip solution. $\mathrm{ES}_{2} \mathrm{~S}_{2}$ tests were performed on the irradiated and thermally treated NG-CSSX solvent. In both cases, the most severe degradation occurred in the presence of the SRS-15 simulant, though the thermal degradation in the presence of $0.025 \mathrm{M} \mathrm{NaOH}$ scrub solution was equally severe. The observed increase in $D_{\mathrm{Cs}}$ values for stripping in both studies indicates that possible impurities formed upon degradation of the solvent will eventually impair stripping. Preliminary GC results show that DCiTG also degrades, which likely contributes to the deterioration of stripping performance. ESI-MS characterization of the degraded solvents enables conclusions concerning the identities of some of the solvent degradation products. It is recommended that further work be carried out to identify the major degradation products and to determine their fates in the flowsheet, supporting a rational choice of wash solution for the wash stages.

\subsubsection{Solvent blend strategy for $\mathrm{MCU}$}

An equal-volume blending strategy has been validated for upgrading freshly prepared BOBCalixC6 CSSX solvent to a blended solvent functionally equivalent to NG-CSSX solvent ${ }^{43}$. Blending fresh BOBCalixC6 CSSX solvent as currently used in MCU with an equal volume of an NG-CSSX solvent concentrate of appropriate composition yields a blended solvent composition (46.5 mM of MaxCalix, $3.5 \mathrm{mM}$ of BOBCalix, $0.5 \mathrm{M}$ of Cs-7SB, $3 \mathrm{mM}$ of guanidine suppressor, and $1.5 \mathrm{mM}$ of TOA in Isopar L) that exhibits equivalent batch ESS performance to that of the NG-CSSX solvent containing $50 \mathrm{mM}$ of MaxCalix, $0.5 \mathrm{M}$ of Cs-7SB, and $3 \mathrm{mM}$ of guanidine suppressor in Isopar L. The solvent blend composition is robust to third-phase formation. Results also show that a blend containing up to $60 \% \mathrm{v} / \mathrm{v}$ of BOBCalixC6 CSSX solvent could be accommodated with minimal risk. Extraction and density data for the effect of solvent concentration mimicking diluent evaporation or over-dilution of the equal-volume blended solvent are also given, providing input for setting operational limits. Given 
that the experiments employed all pristine chemicals, the results do not qualify a blended solvent starting with actual used MCU solvent, which can be expected to have undergone some degree of degradation. Consequently, further work should be considered to test blending with actual MCU used solvent.

\subsubsection{Flowsheet Behavior of NG-CSSX Solvent}

\subsubsection{Optimized flowsheet for MCU}

The NG-CSSX flowsheet applicable for MCU (see Chapter 3) consists of 7 stages of extraction at $\mathrm{O}: \mathrm{A}=1: 4,2$ stages of scrubbing with $0.025 \mathrm{M} \mathrm{NaOH}$ at $\mathrm{O}: \mathrm{A}=3.75: 1$, and 7 stages of stripping with $0.01 \mathrm{M}$ boric acid at $\mathrm{O}: \mathrm{A}=3.75: 1$. The default solvent wash is 2 stages with $0.01 \mathrm{M} \mathrm{NaOH}$ at $\mathrm{O}: \mathrm{A}=$ $3.75: 1$, though there is only a limited basis for this choice. Test data at ORNL and SRNL ${ }^{44}$ and associated calculations (ANL and SRR) to date confirm that this solvent will be able to deliver a minimum cesium DF of 40,000 in the fixed 18-stage configuration of the MCU with modestly increased throughput ${ }^{4}$. The corresponding flowsheet optimized for MCU is shown in Fig. 13.1. It can also provide for higher throughput at SWPF by allowing feed flowrates to be increased, mainly by reducing O:A ratios to as low as 1:10 but also possibly by better hydraulic performance. Because of the high extraction strength of the solvent, a modular deployment at Hanford also becomes possible.

\subsubsection{Model for predicting $D_{\mathrm{Cs}}$ from salt-waste compositions}

In collaboration with Tennessee Technological University (Prof. Dale Ensor), progress was made in developing a model for predicting cesium distribution in extraction from various salt-waste compositions with NG-CSSX solvent (Chapter 7). A large data set of $D_{\mathrm{Cs}}$ values was collected for basic salt mixtures containing a range of concentrations of $\mathrm{Na}^{+}, \mathrm{K}^{+}, \mathrm{NO}_{3}^{-}$, and $\mathrm{OH}^{-}$ions. A simple analog model based on mass action was derived and refined by least squares in a spreadsheet based on four adjustable parameters. The model was shown to predict $D_{\mathrm{Cs}}$ for two waste simulants within ca. $\pm 15 \%$ of the experimental $D_{\mathrm{Cs}}$ values. Further distribution data are being collected at different total salt concentration, and future modeling will be extended with the more sophisticated program SXFIT. 


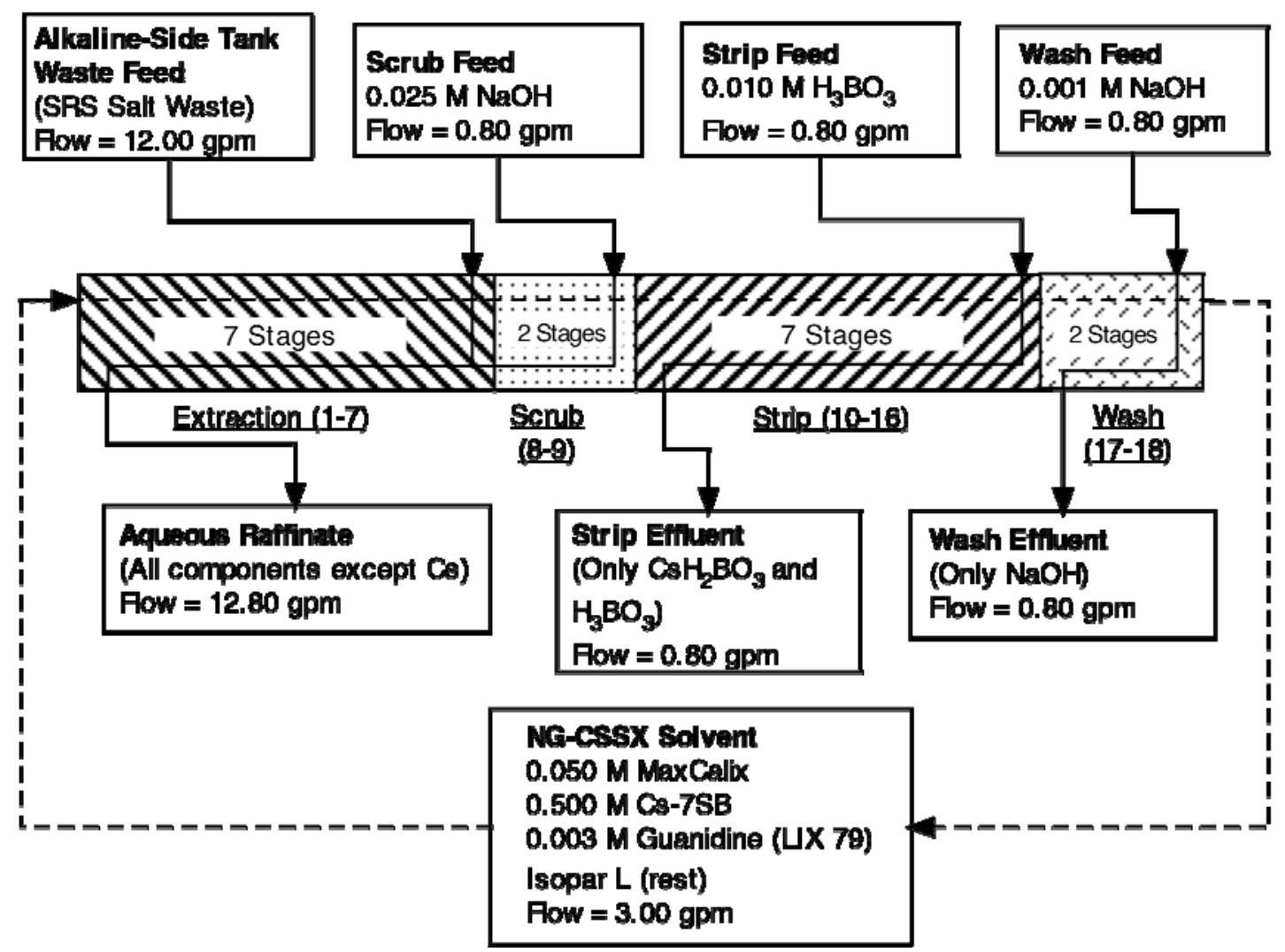

Fig. 13.1. Using simulant test data provided by ORNL as verified by real-waste test data at SRNL, the above flowsheet was calculated to have DF $\geq 40,000$ in the MCU equipment configuration (see Chapter $3^{39}$ ).

\subsubsection{Ten-cycle experiment}

Ten sequential $\mathrm{ES}_{2} \mathrm{~S}_{3}$ experiments were conducted using SRS-15 simulant, showing that the NGCSSX chemistry is robust to a limited number of cycles without significant change in $D_{\mathrm{Cs}}$ values (unreported results).

\subsubsection{ESS testing at high sodium concentration}

A batch ESS experiment using SRS-15 simulant demonstrated that the cesium extraction strength of the NG-CSSX solvent increases with waste concentration above the design feed concentration of 5.6 $M$ sodium, in support of a possible strategy to increase waste-processing rate by increasing salt concentration (see Chapter 8). A series of five SRS simulants were utilized in the experiment with concentrations of sodium ranging from 3.5 to $8 \mathrm{M}$, all other components remaining in the same proportion. Assuming that hydraulics and stage efficiency are not negatively impacted, the simple batch results obtained here would imply that the NG-CSSX process could support an increased wasteprocessing rate based on increased waste concentration while maintaining required waste 
decontamination. Further R\&D is needed to test these assumptions on NG-CSSX performance and to examine system-wide impacts. Factors to be studied include: risk of precipitation of solids in equipment; slower filtration rates at higher viscosity; effects on pump efficiency for transfer; impacts on phase separation of entrained solvent in the more concentrated waste; and formulation of the grout used for final disposal of decontaminated solutions all require exploration.

\subsubsection{Effect of temperature on cesium distribution in ESS batch tests}

A much steeper temperature dependence was found for NG-CSSX in comparison with the currently employed BOBCalixC6 CSSX process in the MCU (see Chapter 8). ESS batch tests were performed with SRS-15 waste simulant using NG-CSSX solvent at 15,25 , and $35^{\circ} \mathrm{C}$. Whereas $D_{\mathrm{Cs}}$ decreases 3.7-fold for the extraction step of BOBCalixC6 CSSX when the temperature is increased from $15{ }^{\circ} \mathrm{C}$ to $35^{\circ} \mathrm{C}, D_{\mathrm{Cs}}$ decreases 11.5 -fold for the extraction step of NG-CSSX over the same temperature range. Scrubbing and stripping in NG-CSSX also show an order-of-magnitude decrease in $D_{\mathrm{Cs}}$ over the same temperature range. It may be concluded that NG-CSSX process performance and predictability depends on the implementation of adequate temperature control and may be deliberately enhanced with lower temperature during extraction and higher temperature in stripping.

\subsubsection{Kinetics of extraction and stripping at $25^{\circ} \mathrm{C}$}

Data obtained in this work show that the extraction and stripping kinetics of the NG-CSSX process are significantly faster than the BOBCalixC6 CSSX process currently in use at the SRS (see Chapter 9). Given that the NG-CSSX process employs a different solvent and a different stripping chemistry as compared with the BOBCalixC6 CSSX process, it was necessary to ensure that the kinetics of NGCSSX are at least as fast as that of BOBCalixC6 CSSX. A series of timed contacts of the NG-CSSX solvent with simulated SRS salt waste were conducted by vortexing up to $60 \mathrm{~s}$. Stripping kinetics were studied for the SRS-15 waste simulant system, with a $0.01 \mathrm{M}$ boric acid strip solution following one contact with SRS-15 feed and two scrub contacts with $0.05 \mathrm{M} \mathrm{NaOH}$. Kinetics of NG-CSSX surpassed that of BOBCalixC6 CSSX in both extraction and stripping, 90\% mass transfer being reached at $7 \mathrm{~s}$ and $9 \mathrm{~s}$, respectively, less than a half and a third of the corresponding times for BOBCalixC6 CSSX solvent. The faster kinetics of NG-CSSX are a promising prerequisite for good mass-transfer efficiency in centrifugal contactors. 


\subsubsection{Effect of solvent impurities on cesium distribution in ESS batch tests}

Results of ESS tests with various impurities added to the NG-CSSX solvent indicated no significant impacts to process performance, at least insofar as the conditions tested (see Chapter 11). A new question posed, regarding the NG-CSSX process relative to the baseline BOBCalixC6 CSSX process now in use in the MCU, is the effect of DCiTG suppressor decomposition products on cesium distribution in ESS. It was found that a set of anticipated guanidine breakdown products at $1 \mathrm{mM}$ initial concentration in the NG-CSSX solvent (i.e., assuming the decomposition of a third of the guanidine with no mitigation) has a negligible effect on ESS performance. It was also shown that the known 4-sec-butylphenol (SBP) decomposition product of the Cs-7SB modifier at a concentration of $10 \mathrm{mM}$ in the NG-CSSX solvent impairs stripping performance, approximately to the same degree as already known for BOBCalixC6 CSSX. The SBP is readily washed from the solvent, and its buildup to such a level is not expected in normal operation. Initial results thus indicate no particular sensitivity to impurities relative to experience with BOBCalixC6 CSSX. However, the actual NG-CSSX degradation products have not yet been identified, and actual partition ratios of the major impurities in the process are not known. Future work is recommended to analyze degraded solvent and to determine the fate and effect of degradation products, as well as to provide a rational basis for choice of a solvent-wash solution.

\subsubsection{Emulsion formation behavior}

Tests show that the NG-CSSX has a low risk of problems from emulsion formation. However, formation of a thick, stable emulsion occurs on contact of NG-CSSX solvent with boric acid solutions when DCiTG in the solvent and the aqueous boric acid concentration exceed threshold concentrations (unreported results). At the $0.003 \mathrm{M}$ DCiTG concentration employed in the solvent, emulsion formation was not observed at any boric acid concentration at $25{ }^{\circ} \mathrm{C}$, and thus, there appears to be minimal risk to the NG-CSSX process solvent and flowsheet as designed. As the aqueous boric acid concentration is raised at a given DCiTG concentration, the interfacial tension is observed to drop dramatically as the emulsion-formation threshold is approached. The emulsion can be easily broken on contact with certain aqueous solutions. Further characterization of emulsion formation and conditions for breaking the emulsion applicable to process use is warranted, as emulsions lead to process upset and not all needed data have been acquired. In particular, the temperature dependence is needed, as stripping is carried out at $33 \pm 3{ }^{\circ} \mathrm{C}$, and the data were obtained at $25^{\circ} \mathrm{C}$. In addition, the relationship of guanidine structure and emulsion formation is of interest in that a more lipophilic alternative to DCiTG is a recommended change to the solvent composition ${ }^{6}$. 


\subsubsection{Acid-base behavior of the NG-CSSX solvent}

A limited amount of data have been collected on the protonation behavior of DCiTG and the distribution of boric acid and other acids to the NG-CSSX solvent in the stripping stage (unreported data). It is important to acquire an improved understanding of stripping chemistry, as it is currently not well understood and, yet, is very sensitive to process conditions. In addition, acid equivalents carried by the solvent from stripping to the wash section will neutralize the wash solution. It is desirable to know the extent of this neutralization in order to set effective wash conditions properly. It appears that borate species are not extracted into the solvent in the stripping step. However, this is known only from negative results from nuclear magnetic resonance (NMR) spectroscopy and ESI-MS experiments.

\subsubsection{Fate of metals in extraction, scrubbing, and stripping}

Results of ICP-AES analysis of scrub and strip aqueous phases in a standard ESS test with SRS-15 feed showed that the scrub section is effective at removing the major competing metals sodium and potassium (Section 8.10). Only traces of these metals reach stripping, which appears to produce a fairly pure stream of cesium in dilute boric acid. Other metals such as aluminum do not report to the scrub or strip solutions. An analysis of the scrub and strip solutions for the major anions nitrate and nitrite is desirable, as it is known that nitrate especially deteriorates stripping performance.

\subsubsection{Effect of feed cesium concentration on cesium distribution in ESS batch tests}

The primary effect of cesium loading was observed to be significantly increased stripping $D_{\mathrm{Cs}}$ values, but within acceptable limits under anticipated process bounds at the SRS. The effect of cesium loading up to, and in excess of, the maximum expected loading under SRS conditions was determined in standard ESS tests (unreported data). Loading at the level expected for low-curie feeds to the MCU is seen to have only a small impact on stripping, while the maximum cesium concentration expected in SWPF feed would raise stripping $D_{\mathrm{Cs}}$ values an order of magnitude. While stripping is still performing satisfactorily at the maximum expected cesium loading, the partial loss of stripping capacity means that the margin for deterioration of stripping performance due to stresses of solvent degradation and impurities buildup is diminished. Given that NG-CSSX is currently optimized for MCU conditions, it is recommended that the loading issue be examined further to determine whether an adjustment to the NG-CSSX flowsheet might be warranted for SWPF. A possible approach is to raise the boric acid concentration of the strip solution to $15-20 \mathrm{mM}$ to increase the capacity of the 
boric acid to absorb the added cesium hydroxide equivalents. Information on the temperature dependence of stripping would be useful, since stripping is carried out at $33 \pm 3{ }^{\circ} \mathrm{C}$.

\subsubsection{Effect of O:A ratio on cesium distribution in ESS batch tests}

The O:A ratio on stripping can be raised to $10: 1$ with only minor increase in stripping $D_{\mathrm{Cs}}$ values, allowing process operation to take place with variable CF up to at least 40 (unreported data). Because the O:A ratio on extraction has only a minor effect on extraction, it was not systematically examined. However, on stripping, the increased transfer of cesium into the strip solution as the stripping O:A ratio is is increased, is expected to have a similar effect as an increase in cesium feed concentration (see above). The flexibility of O:A in stripping means that there is the possibility that process operators could adjust the CF so that the strip effluent curie content can be maintained at a constant value, thereby minimizing water delivery to, and concomitant evaporation burden on, the Defense Waste Processing Facility.

\subsubsection{Physical Properties of the NG-CSSX Solvent and 5-cm Contactor Performance}

\subsubsection{Property determinations}

Results of dispersion-number determinations indicate that all solution pairs considered for use in extraction, scrubbing, and stripping operations can be separated adequately in centrifugal contactors. Decreases in Cs-7SB concentration were found to have a consistent effect of improving phase separation behavior across the process cascade. Dispersion numbers for stripping operations using $\mathrm{H}_{3} \mathrm{BO}_{3}$ at a concentration of $1 \mathrm{mM}$ are undesirably low; it is recommended that minimum acid concentration of $10 \mathrm{mM} \mathrm{H}_{3} \mathrm{BO}_{3}$ be used in stripping. As expected, dispersion number results are consistent with interfacial tension data, in that higher values of the later (indicating greater difficulty in interface breakage) were obtained for systems having higher dispersion numbers (indicating greater tendency for dispersion separation).

\subsubsection{Contactor hydraulics}

Phase separation was good over a fairly wide range of conditions, but not at throughputs approaching the vendor-claimed processing capacity of $>2 \mathrm{~L} / \mathrm{min}$. Under extraction conditions, performance was acceptable for the $50 \mathrm{mM}$ MaxCalix solvent formation at the phase ratios considered for use in the MCU at flow rates less than half the manufacturer's claims, assuming the continued use of coalescers for removal of minor levels of organic entrainment. Acceptable separation under scrubbing and stripping conditions was limited to throughputs below $600 \mathrm{~mL} / \mathrm{min}$, total flow. It is important to note 
that aqueous samples were found to be free of visible organic contamination in all cases, and that increases in organic contamination of aqueous effluents were observed as a function of time (with all other factors being constant), interpreted as an approach to steady state. The limitations in throughput are believed to be due to design features of the commercial contactor used and could be mitigated by modifications tested as part of NG-CSSX development.

\subsubsection{Mass transfer}

Mass-transfer efficiencies for extraction and stripping indicate high likelihood of success in a possible transition from BOBCalixC6 CSSX to NG-CSSX processing using the contactors in place in the MCU and SWPF. The extraction efficiencies are higher than those obtained using the BOBCalixC6 CSSX solvent. Once steady state was reached, the NG-CSSX solvent had efficiencies in the range 98-99\%, compared with $86-89 \%$ for BOBCalixC6 CSSX. The improved stage efficiencies in extraction should easily support the target DF of $\geq 40,000$, as flowsheet calculations had earlier assumed $\geq 90 \%{ }^{3}$. In the previous work using the BOBCalixC6 CSSX solvent, cesium partitioning behavior under the scrub condition was not determined. The NG-CSSX system had relatively poor stage efficiencies, in the range $25-49 \%$, with the lower value recorded before steady state had been reached. It should be borne in mind, however, that scrubbing stage efficiencies probably should be defined more in terms of potassium or sodium distribution, given the main purpose of the scrub stages in removing these metals. Stage efficiencies obtained under stripping conditions (in the range $72-$ $75 \%$ ) were lower than those obtained in NG-CSSX extraction or in BOBCalixC6 CSSX stripping (94-100\%), but are still considered acceptable, given the very low $D_{\mathrm{Cs}}$ values in stripping. The lower mass-transfer efficiencies measured for NG-CSSX contrast with the faster kinetics (see Chapter 9). Additional mass transfer testing at various flow rates (residence times) would be of benefit, particularly under scrubbing and stripping conditions, in determining if these efficiency values are rate dependent.

\subsubsection{Air flow and entrainment evaluations and contactor modifications}

Testing performed using a variety of internal vanes - full diameter, partial diameter, and no vanesindicate the generation of a slight negative pressure in the air space located along the centerline of the rotor. Further, the source of the pressure effect appears to be the configuration of the light-phase discharge ports and the upper end of the rotor, as the effect was present when no vanes were in place. The pressure effect includes a slight regular and periodic fluctuation that is consistent with liquid pulsing in the mixing zone of the contactor. The combined observations tend to explain the 
evaporation of solvent components during operation, which results in a minor transient condition that requires supplemental addition of these components. Elimination of the pressure effect and the flow it induces would require a design modification of some significance.

The transfer of less-dense organic-phase material into the heavy-phase collector ring was indicated during testing. Corresponding transfer of heavy phase material (aqueous solution) in the opposing collector ring was not. The difference is more likely a reflection of the fact that the light-phase discharge is actually a discharge of both liquid and gas and that the organic material used has a lower surface tension than the aqueous solution, than it is the result of any difference in configuration between the two collector rings. Increasing throughput tended to exacerbate the problem, while an increase in rotor speed had minimal effect, if any. This observation is consistent with the mitigation of entrainment issues in MCU by reduction of processing throughput.

A modification to the collector ring did result in a consistent and significant reduction in the quantity of organic solution transferred into the aqueous flow path. At a minimum (within the data scatter), a 15 -fold reduction in carryover is indicated.

\subsection{CONCLUSIONS AND RECOMMENDATIONS}

In the itemized summary presented above, the NG-CSSX process chemistry has been shown to provide remarkable added value for salt-waste processing in comparison with the current baseline BOBCalixC6 CSSX process. Benefits include markedly higher DF, from a design value of 12 in the MCU to $\geq 40,000$ in the same equipment, together with enhanced throughput. Although it is the equipment that must deliver the throughput - full-scale contactor tests at the SRS are successfully addressing the question of greater throughput - the chemistry alone supports a higher throughput by allowing a large decrease in O:A ratio on extraction, meaning aqueous feed flow can be increased at constant total contactor throughput. In addition, salt concentration can potentially be increased to obtain another major gain in waste-processing rate. A further benefit obtainable from NG-CSSX is a higher CF, which the data show can easily reach a value of 40 in comparison with a nominal value of 15 commonly adopted at the SRS. All of these benefits can markedly improve waste-processing performance at the SRS (both MCU and SWPF) as well as at Hanford.

In more specific terms regarding work performed, the NG-CSSX solvent has been optimized and characterized, a flowsheet has been developed and characterized in detail specifically for MCU deployment, and 5-cm contactor performance including a variety of device modifications has been 
determined. Extraction, scrubbing, and stripping are robust over a range of feed compositions, flowsheet parameters, and stresses due to thermal and radiation degradation. Solvent performance is projected to be stable to expected thermal and radiation stresses for up to 3 years of operation. Preparative methods for the guanidine suppressor DCiTG have been developed along with an analytical method based on GC. Properties of DCiTG, including effective stripping and adequate stability have been measured; partitioning loss to the aqueous strip solution is not as low as desired, though this issue can be readily dealt with by added process monitoring and solvent trimming or by use of a more lipophilic guanidine. Methods have also been developed for straightforward preparation of alternative guanidines. Flowsheet design provides for a DF $\geq 40,000$ in the 18-stage MCU configuration of centrifugal contactors with at least a modest increase in throughput. Although the NG-CSSX solvent delivers improved physical properties and faster kinetics than the baseline BOBCalixC6 CSSX solvent, 5-cm contactor testing indicates that throughput and mass transfer may be more a matter of contactor design and dynamics than it is solvent chemistry and physical properties. Nevertheless, powerful extraction and stripping of NG-CSSX can deliver higher throughput even without better contactor hydraulics.

Based on the results of the ORNL research in the past 18 months, further development and testing are recommended to add further value to NG-CSSX process and to fill gaps in understanding of the process, permitting better predictability and control of the process:

1) An alternative, more lipophilic guanidine suppressor to replace DCiTG would reduce the cost of solvent monitoring and improve ease of analysis.

2) The identity of solvent degradation products should be determined along with their fate in the process cascade. These data should be used to make an informed choice of solvent-wash solution. The effect of nitric acid on solvent stability should also be investigated, as nitric acid is a common process chemical and contactor cleaning agent.

3) Optimization of the solvent composition, aqueous compositions, and flowsheet design should be considered for SWPF and for any Hanford application. NG-CSSX has been developed for MCU deployment and may not be optimum for other applications. This study should include options to achieve much higher CFs in order to minimize the evaporation burden in vitrification.

4) The potential for a higher waste-processing rate by increasing salt concentration should be examined. Impacts of high sodium concentration on the performance of process chemistry, contactor hydraulics and mass transfer need to be determined, as well as the performance of the SRS systemwide waste processing. 
5) Elucidate emulsion-formation conditions as a function of temperature to $35{ }^{\circ} \mathrm{C}$ and design better methods for breaking the emulsion should it form.

6) Since solvent blending has only been validated for blending of fresh chemicals, the blending of actual used MCU solvent needs to be validated.

7) Further development of a process model for prediction of $D_{\mathrm{Cs}}$ as a function of feed composition is needed for applicability to all waste types.

8) Improved understanding of stripping and acid-base reactions in scrubbing, stripping, and washing is desirable. The fate of anions in scrubbing and stripping is also not understood.

9) An improved understanding of the effect of temperature on ESS performance at high cesium and potassium feed concentrations is desirable for predictability of processing performance of maximum curie feeds at the SWPF and for high potassium feeds at Hanford.

10) Additional mass-transfer testing at various flow rates would be of benefit, particularly under scrubbing and stripping conditions, in determining if efficiency values are rate dependent.

\subsection{PUBLICATIONS, REPORTS, AND PRESENTATIONS RESULTING FROM THIS RESEARCH AND DEVELOPMENT PROGRAM}

\subsubsection{Publications}

B. A. Moyer, P. V. Bonnesen, L. H. Delmau, F. V. Sloop, Jr., N. J. Williams, J. F. Birdwell, Jr., D. L. Lee, R. A. Leonard, S. D. Fink, T. B. Peters, and M. W. Geeting, "Development of the NextGeneration Caustic-Side Solvent Extraction (NG-CSSX) Process for Cesium Removal from High Level Tank Waste," Paper 11346, Proc. Waste Management 2011, February 27-March 3, 2011, Phoenix, AZ; Waste Management Symposia, Tempe, AZ (2011).

L. H. Delmau, J. F. Birdwell, Jr., P. V. Bonnesen, N. B. Ladd, B. A. Moyer, E. L. Stoner, D. L. Lee, F. V. Sloop, Jr., "Next-Generation Caustic-Side Solvent Extraction (NG-CSSX) Process," Proc. International Solvent Extraction Conference ISEC 2011, Oct. 3-7, 2011, Santiago, Chile.

N. C. Duncan, B. D. Roach, N. J. Williams, P. V. Bonnesen, A. Rajbanshi, B. A. Moyer, N,N'Dicyclohexyl-N"-Isotridecylguanidine as Suppressor for the Next Generation Caustic Side Solvent Extraction (NG-CSSX) Process, Sep. Sci. Technol. 47 (14-15), 2074-2087 (2012).

\subsubsection{Reports (ORNL)}

B. A. Moyer, "Initial Next-Generation Caustic-Side Solvent Extraction (NG-CSSX) Process Solvent Recommendation," ORNL LTR-NGCSSX-001, Oak Ridge National Laboratory, Oak Ridge, TN, June 30, 2010.

B. A. Moyer, "Optimized Next-Generation Caustic-Side Solvent Extraction (NG-CSSX) Process Solvent Composition," ORNL LTR-NGCSSX-002, Oak Ridge National Laboratory, Oak Ridge, TN, September 30, 2010. 
P. V. Bonnesen, "Purity Specifications for 1,3-alt-25,27-di(3,7-dimethyloctyl-1-oxy)calix[4]arenebenzocrown-6 ('MaxCalix')," ORNL LTR-NGCSSX-003, Oak Ridge National Laboratory, Oak Ridge, TN, February 2011.

J. F. Birdwell and D. L. Lee, "Property Determinations and Preliminary Engineering Evaluation of Next Generation CSSX Processing," ORNL LTR-NGCSSX-004, Oak Ridge National Laboratory, Oak Ridge, TN, December 30, 2010.

B. A. Moyer, P. V. Bonnesen, L. H. Delmau, F. V. Sloop, Jr., N. J. Williams, J. F. Birdwell, Jr., D. L. Lee, R. A. Leonard, S. D. Fink, T. B. Peters, and M. W. Geeting, "Development of the NextGeneration Caustic-Side Solvent Extraction (NG-CSSX) Process for Cesium Removal from High Level Tank Waste," ORNL LTR-NGCSSX-005, Oak Ridge National Laboratory, Oak Ridge, TN, March 2011; Paper 11346, Proceedings Waste Management 2011, February 27-March 3, 2011, Phoenix, AZ; Waste Management Symposia, Tempe, AZ (2011).

P. V. Bonnesen and N. C. Duncan, "Minimum Purity Requirements for 1,3-Dicyclohexyl-2(isotridecyl)guanidine and 1,3-Dicyclohexyl-2-(isotridecyl)guanidine Hydrochloride (DCiTG and DCiTG $\bullet \mathrm{HCl}$, Suppressor for Next Generation CSSX Process Solvent)," ORNL LTR-NGCSSX-006, Oak Ridge National Laboratory, Oak Ridge, TN, February 2011.

P. V. Bonnesen, N. C. Duncan, and A. Rajbanshi, "Preparation of 1,3-dicyclohexyl-2(isotridecyl)guanidine and 1,3-dicyclohexyl-2-(isotridecyl)guanidine hydrochloride (DCiTG and DCiTG.HCl, suppressor for Next Generation CSSX process solvent)," ORNL LTR-NGCSSX-007, Oak Ridge National Laboratory, Oak Ridge, TN, April 2011.

N. J. Williams and B. A. Moyer, "Effect of High Sodium Levels on Cesium Distribution in the Next Generation Caustic Side Solvent Extraction (NG-CSSX) Process," ORNL LTR-NGCSSX-008, Oak Ridge National Laboratory, Oak Ridge, TN, August 5, 2011.

J. F. Birdwell, Jr., D. L. Lee, and P. L. Szczygiel, "Next Generation CSSX Mass Transfer Performance in a Partially Pumping 5-cm Centrifugal Contactor," ORNL LTR-NGCSSX-009, Oak Ridge National Laboratory, Oak Ridge, TN, March 31, 2011.

L. H. Delmau and B. A. Moyer, "Solvent Blending Strategy to Upgrade MCU CSSX Solvent to Equivalent Next-Generation CSSX Solvent," ORNL LTR-NGCSSX-010, Oak Ridge National Laboratory, Oak Ridge, TN, July 22, 2011.

N. C. Duncan and P. V. Bonnesen, "Method Development for the Gas Chromatography (GC) Analysis of 1,3-Dicyclohexyl-2-(isotridecyl)guanidine (DCiTG, Suppressor for Next Generation CSSX Process Solvent)," ORNL LTR-NGCSSX-011, Oak Ridge National Laboratory, Oak Ridge, TN, April 2011.

N. J. Williams and B. A. Moyer, "Temperature Dependence of the Next-Generation Caustic Side Solvent Extraction (NG-CSSX) Process Solvent," ORNL LTR-NGCSSX-012, Oak Ridge National Laboratory, Oak Ridge, TN, August 5, 2011.

B. D. Roach, N. J. Williams, N. C. Duncan, and B. A. Moyer, "Effect of Possible Impurities on the Performance of the Next-Generation Caustic Side Solvent Extraction (NG-CSSX) Process," ORNL LTR-NGCSSX-013, Oak Ridge National Laboratory, Oak Ridge, TN, August 19, 2011.

N. J. Williams and B. A. Moyer, "Kinetics of Cesium Extraction and Stripping in the Next Generation 
Caustic Side Solvent Extraction (NG-CSSX) Process," ORNL LTR-NGCSSX-014, Oak Ridge National Laboratory, Oak Ridge, TN, September 16, 2011.

J. F. Birdwell, D. L. Lee, and S. H. Bruffey, "Property Determinations and Preliminary Engineering Evaluation of Next Generation CSSX Processing," ORNL LTR-NGCSSX-015, Oak Ridge National Laboratory, Oak Ridge, TN, September 30, 2011.

L. H. Delmau, J. F. Birdwell, Jr., P. V. Bonnesen, N. B. Ladd, B. A. Moyer, E. L. Stoner, D. L. Lee, F. V. Sloop, Jr., "Next-Generation Caustic-Side Solvent Extraction (NG-CSSX) Process," ORNL LTR-NGCSSX-016, Oak Ridge National Laboratory, Oak Ridge, TN, September 30, 2011; Proc. International Solvent Extraction Conference ISEC 2011, Oct. 3-7, 2011, Santiago, Chile.

N. J. Williams, B. D. Roach, B. A. Moyer, and D. L. Lee, "Effect of Radiolytic and Thermal Treatment on the Performance of the Next-Generation Caustic-Side Solvent Extraction (NG-CSSX) Solvent," ORNL LTR-NGCSSX-017, Oak Ridge National Laboratory, Oak Ridge, TN, September 30, 2011.

B. D. Roach, N. J. Williams, N. C. Duncan, and B. A. Moyer, "N, $N$ '-Dicyclohexyl- $N "-$ isotridecylguanidine Suppressor Variants for the Next Generation Caustic Side Solvent Extraction (NG-CSSX) Process," ORNL LTR-NGCSSX-018, Oak Ridge National Laboratory, Oak Ridge, TN, September 30, 2011.

B. A. Moyer, "Development of the Next Generation Caustic Side Solvent Extraction (NG-CSSX) Process: ORNL Accomplishments in FY 2010 and 2011," ORNL LTR-NGCSSX-019, Oak Ridge National Laboratory, Oak Ridge, TN, September 30, 2011.

B.A. Moyer, L.H. Delmau, N.C. Duncan, D.D. Ensor, T.G. Hill, D.L. Lee, B.D. Roach, F.V. Sloop, Jr., N.J. Williams, "Recommended DCiTGnidine Suppressor for the Next-Generation Caustic-Side Solvent Extraction Process," ORNL/TM-2012/625. Oak Ridge National Laboratory, Oak Ridge, TN. 2013.

B. A. Moyer, J. Birdwell, L. H. Delmau, N. C. Duncan, D. Ensor, T. Hill, D. Lee, B. D. Roach, F. V. Sloop, Jr., E. Stoner and N. J. Williams, "Next Generation Solvent Development for Caustic-Side Solvent Extraction of Cesium," ORNL / TM-2013/136, Oak Ridge National Laboratory, Oak Ridge, TN, April 15, 2013. (This report)

\subsubsection{Report (ANL via subcontract)}

R. A. Leonard, "Initial Flowsheet for the Next Generation CSSX Process in the MCU, Report for FY2010," ANL LTR-NGCSSX-001, Argonne National Laboratory, Argonne, IL, September 30, 2010.

\subsubsection{Presentations}

N. C. Duncan, A. Rajbanshi, N. J. Williams, B. D. Roach, P. V. Bonnesen, B. A. Moyer, "Synthesis and Evaluation of DCiTGnidine Suppressors Used in the Next Generation Caustic Side Solvent Extraction (NG-CSSX) Process," $17^{\text {th }}$ Symposium on Separation Science and Technology for Energy Applications, Gatlinburg, TN, Oct. 23-27, 2011. 
L. H. Delmau, J. F. Birdwell, Jr., P. V. Bonnesen, N. B. Ladd, B. A. Moyer, E. L. Stoner, D. L. Lee, F. V. Sloop, Jr., "Development of the Next-Generation Caustic-Side Solvent Extraction (NG-CSSX) Process," $17^{\text {th }}$ Symposium on Separation Science and Technology for Energy Applications, Gatlinburg, TN, Oct. 23-27, 2011.

L. H. Delmau, J. F. Birdwell, Jr., P. V. Bonnesen, N. B. Ladd, B. A. Moyer, E. L. Stoner, D. L. Lee, F. V. Sloop, Jr., "Next-Generation Caustic-Side Solvent Extraction (NG-CSSX) Process," Proc. International Solvent Extraction Conference ISEC 2011, Oct. 3-7, 2011, Santiago, Chile.

B. D. Roach, N. J. Williams, N. C. Duncan, L. H. Delmau, B. A. Moyer, D. L. Lee, J. F. Birdwell, Jr., "Thermal and Radiolytic Stability of the Next-Generation Caustic-Side Solvent Extraction (NGCSSX) Solvent," International Solvent Extraction Conference ISEC 2011, Oct. 3-7, 2011, Santiago, Chile.

B. A. Moyer, P. V. Bonnesen, L. H. Delmau, F. V. Sloop, Jr., N. J. Williams, J. F. Birdwell, Jr., D. L. Lee, R. A. Leonard, S. D. Fink, T. B. Peters, and M. W. Geeting, "Development of the NextGeneration Caustic-Side Solvent Extraction (NG-CSSX) Process for Cesium Removal from High Level Tank Waste," Paper 11346, Proc. Waste Management 2011, February 27-March 3, 2011, Phoenix, AZ; Waste Management Symposia, Tempe, AZ. 2011.

B. A. Moyer, J. F. Birdwell, Jr.; L. H. Delmau, D. L. Schuh, E. L. Stoner, N. J. Williams, S. D. Fink, T. B. Peters, R. A. Pierce, R. A. Leonard, M. W. Geeting. Next-Generation Cesium Solvent, EM Technical Exchange Meeting, Atlanta, GA, Nov. 16-18, 2010. 



\section{APPENDIX A. QUALITY ASSURANCE IMPLEMENTATION FOR ORNL/TM-2013/224 REV 1}

In terms of the applicable NQA-1 based requirements, Table 1 identifies the corresponding ORNL QA criterion/criteria. ORNL employs an electronic Standard Based Management System (SBMS) to deliver procedures that implement the criteria. Procedures are organized by subject areas under management systems and are implemented using a graded approach appropriate for the work. Documents that tailor the ORNL criteria to the project also appear in the table.

\begin{tabular}{|c|c|c|c|}
\hline \multicolumn{4}{|c|}{ Table A.1 NQA-1 Based Criteria } \\
\hline Implementing Procedures & Document No. & YES & NO \\
\hline Organization & $\begin{array}{l}\text { QAPD (criteria 1, 10); } \\
\text { QAP-X-94-CSD-001 }\end{array}$ & $\mathrm{X}$ & \\
\hline Quality Assurance Program & $\begin{array}{l}\text { QAPD (criteria } \\
1,2,3,9,10 \text { ); QAP-X-94- } \\
\text { CSD-001 }\end{array}$ & $\mathrm{X}$ & \\
\hline Control of Research and Development Activities & QAP-X-94-CSD-001 & $\mathrm{X}$ & \\
\hline $\begin{array}{l}\text { QA Program Requirements for Analytical Measurement } \\
\text { Systems }\end{array}$ & QAP-X-94-CSD-001 & $\mathrm{X}$ & \\
\hline Design Control & N/A & & \\
\hline Procurement Document Control & $\begin{array}{l}\text { QAPD (criterion 7); } \\
\text { QAP-X-94-CSD-001 }\end{array}$ & $\mathrm{X}$ & \\
\hline Instructions, Procedures and Drawings & $\begin{array}{l}\text { QAPD (criteria 4,5); } \\
\text { QAP-X-94-CSD-001 }\end{array}$ & $\mathrm{X}$ & \\
\hline Document Control & $\begin{array}{l}\text { QAPD (criterion 4); } \\
\text { QAP-X-94-CSD-001; } \\
\text { CSD-OP-000-AD01 }\end{array}$ & $\mathrm{X}$ & \\
\hline Control of Purchased Items and Services & $\begin{array}{l}\text { QAPD (criterion 7); } \\
\text { QAP-X-94-CSD-001 }\end{array}$ & $\mathrm{X}$ & \\
\hline Identification and Control of Items & $\begin{array}{l}\text { QAPD (criteria 5, 8); } \\
\text { QAP-X-94-CSD-001 }\end{array}$ & $\mathrm{X}$ & \\
\hline Control of Processes & $\begin{array}{l}\text { QAPD (criterion 5); } \\
\text { QAP-X-94-CSD-001 }\end{array}$ & $\mathrm{X}$ & \\
\hline Control of Nondestructive Examination & N/A & & \\
\hline Control of Welding and Other Joining Processes & $\mathrm{N} / \mathrm{A}$ & & \\
\hline Work Planning and Control & QAPD (criterion 5) & $\mathrm{X}$ & \\
\hline Inspection & $\begin{array}{l}\text { QAPD (criteria 8, 10); } \\
\text { QAP-X-94-CSD-001 }\end{array}$ & $\mathrm{X}$ & \\
\hline Test Control & $\begin{array}{l}\text { QAPD (criteria 8, 10); } \\
\text { QAP-X-94-CSD-001 }\end{array}$ & $\mathrm{X}$ & \\
\hline Control of Measuring and Test Equipment & $\begin{array}{l}\text { QAPD (criteria 5, 8); } \\
\text { QAP-X-94-CSD-001 }\end{array}$ & $\mathrm{X}$ & \\
\hline Control of Installed Process Instrumentation & $\begin{array}{l}\text { QAPD (criterion 5); } \\
\text { QAP-X-94-CSD-001 }\end{array}$ & $\mathrm{X}$ & \\
\hline Packaging, Handling, Shipping and Storage & $\begin{array}{l}\text { QAPD (criterion 5); } \\
\text { QAP-X-94-CSD-001 }\end{array}$ & $\mathrm{X}$ & \\
\hline
\end{tabular}




\section{Table A.1 NQA-1 Based Criteria}

\begin{tabular}{|c|c|c|c|}
\hline Implementing Procedures & Document No. & YES & NO \\
\hline $\begin{array}{l}\text { Notes: } \\
\text { QAPD - ORNL Quality Assurance Program Description - des } \\
\text { flowed into implementing procedures through subject areas de } \\
\text { QAP-X-94-CSD-001 - Chemical Sciences Division Quality As } \\
\text { within the Chemical Sciences Division. Criterion } 5 \text { includes se } \\
\text { registered research notebook process. The handling of register } \\
\text { exhibit: Instructions for Use of this Research and Technical N }\end{array}$ & $\begin{array}{l}\text { are parsed to management } \\
\text { t systems. } \\
\text { how the ORNL subject are } \\
\text { cumentation of research d } \\
\text { escribed in the SBMS Rec } \\
\text { are handled as permanent. }\end{array}$ & $\begin{array}{l}\text { Is and } \\
\text { graded } \\
\text { ng the } \\
\text { ibject area }\end{array}$ & \\
\hline
\end{tabular}




\section{APPENDIX B. ORNL PUBLICATION TRACKING SYSTEM APPROVALS}

\begin{tabular}{|c|c|c|c|c|c|}
\hline Pub ID & \multicolumn{5}{|l|}{44254} \\
\hline Title & \multicolumn{5}{|c|}{ Next Generation Solvent Development for Caustic-Side Solvent Extraction of Cesium } \\
\hline Status & \multicolumn{5}{|l|}{ Distributed } \\
\hline ORNL Review? & \multicolumn{5}{|c|}{ Scientific communication that requires ORNL review } \\
\hline Information Category & \multicolumn{5}{|l|}{ Unlimited } \\
\hline ORNL Report Classification & \multicolumn{5}{|l|}{ Other } \\
\hline Contact Person & \multicolumn{5}{|c|}{ Sloop Jr, Frederick $\{$ Fred $\} \mathrm{V}$} \\
\hline Responsible Organization & \multicolumn{5}{|c|}{ Chemical Sciences Division (50000105) } \\
\hline Prepared at & \multicolumn{5}{|c|}{ This scientific communication is being prepared by someone at ORNL. } \\
\hline Document Access & \multicolumn{5}{|l|}{ Available to the public. } \\
\hline Authors & \multicolumn{2}{|c|}{$\begin{array}{l}\text { Duncan, Nathan C. } \\
\text { Delmau, Laetitia Helene } \\
\text { Ensor, Dale } \\
\text { Lee, Denise L. } \\
\text { Birdwell Jr, Joseph F. } \\
\text { Hill, Talon G. } \\
\text { Williams, Neil J. } \\
\text { Stoner, Erica L. } \\
\text { Roach, Benjamin D. } \\
\text { Moyer, Bruce A. } \\
\text { Sloop Jr, Frederick \{Fred\} V. }\end{array}$} & $\begin{array}{l}\text { ORNL }(956737 \\
\text { ORNL (37967) } \\
\text { Tennessee Tec } \\
\text { ORNL }(923397 \\
\text { ORNL }(23072) \\
\text { ORNL }(300330 \\
\text { ORNL }(977118 \\
\text { ORNL }(965527 \\
\text { ORNL }(985945 \\
\text { ORNL }(22206) \\
\text { ORNL }(34531)\end{array}$ & ological University & \\
\hline Workflow & $\begin{array}{l}06 / 24 / 201308: 40: 27 \\
06 / 24 / 201318: 42: 13 \\
06 / 24 / 201318: 42: 13 \\
06 / 24 / 201318: 39: 49 \\
06 / 25 / 201308: 58: 00 \\
06 / 28 / 201311: 42: 14 \\
06 / 28 / 201311: 50: 41 \\
06 / 28 / 201314: 15: 24 \\
07 / 11 / 201308: 50: 29 \\
07 / 11 / 201314: 08: 59 \\
07 / 11 / 201314: 08: 59 \\
07 / 11 / 201314: 38: 04\end{array}$ & $\begin{array}{l}\text { Draf } \\
\text { Auth } \\
\text { Sub } \\
\text { Sup } \\
\text { Deri } \\
\text { Exp } \\
\text { Tech } \\
\text { Tech } \\
\text { Sup } \\
\text { Divi } \\
\text { Awa } \\
\text { Dist }\end{array}$ & $\begin{array}{l}\text { or Certification } \\
\text { nitted for review } \\
\text { rvisor } \\
\text { vative Classifier } \\
\text { rt control check } \\
\text { nical Reviewer } \\
\text { nical Editor } \\
\text { rvisor } \\
\text { ion Approver } \\
\text { ting Distribution } \\
\text { ibuted }\end{array}$ & $\begin{array}{l}\text { Sloop Jr, Frederick }\{\text { Fred }\} \vee \text { by Lord, Kathryn Lee } \\
\text { Sloop Jr, Frederick }\{\text { Fred\} } \vee \\
\text { Sloop Jr, Frederick }\{\text { Fred\} } \vee \\
\text { Moyer, Bruce A } \\
\text { Hamrin, David Robert } \\
\text { Migun, Rolf P } \\
\text { McFarlane, Joanna } \\
\text { Sloop Jr, Frederick }\{\text { Fred\} } \vee \\
\text { Moyer, Bruce A } \\
\text { Britt, Phillip F } \\
\text { Britt, Phillip F } \\
\text { Sloop Jr, Frederick }\{\text { Fred }\}\end{array}$ & $\begin{array}{l}\text { Auto-Approved } \\
\text { Approved } \\
\text { Approved } \\
\text { Recommended } \\
\text { Recommended } \\
\text { Approved } \\
\text { Approved }\end{array}$ \\
\hline
\end{tabular}


A-6 


\section{APPENDIX C. DCiTG GUANIDINES}

The following appendix is supplementary information for the journal article " $N, N$ '-DICYCLOHEXYL$N "$ "ISOTRIDECYLGUANIDINE AS SUPPRESSOR FOR THE NEXT GENERATION CAUSTIC SIDE SOLVENT EXTRACTION (NG-CSSX) PROCESS" published in separation science and technology in 2012 (reference 21 in main document) ${ }^{1}$.

This work was undertaken to investigate the importance of the purity, concentration, and source of the $N, N^{\prime}$-dicyclohexyl- $N "$-isotridecylguanidine (DCiTG) suppressor (guanidine) used in the NG-CSSX process and their implications for solvent effectiveness. In batch extract/scrub/strip (ESS) tests, it is shown that the source of and, to a certain extent, the purity of the guanidine has little effect on the cesium distribution behavior of the NG-CSSX solvent for three simulants of tank salt waste. The simulants included average SRS salt waste simulant (SRS-15, $\left[\mathrm{K}^{+}\right]=0.015 \mathrm{M}$ ), SRS salt waste simulant at the bounding potassium concentration (SRS-45, $\left[\mathrm{K}^{+}\right]=0.045 \mathrm{M}$ ), and Hanford tank 241-AP-108 simulant with a moderately high potassium concentration $\left(\left[\mathrm{K}^{+}\right]=0.174 \mathrm{M}\right)$. It is also shown that the concentration of the suppressor (nominally $3 \times 10^{-3} \mathrm{M}$ ) could drop to as low as $2.5 \times 10^{-4} \mathrm{M}$ before stripping performance is significantly impaired. While the potassium concentration in the feed apparently does not affect the function of the guanidine suppressor in stripping, the addition of the surfactant sodium dodecylsulfate (SDS) to the SRS-15 simulant has a strong effect, revealing a range of abilities among the DCiTG candidates to suppress the effect of rogue surfactant anions on stripping. The significant partitioning of all of the tested DCiTG suppressors to the strip solution as measured by gas chromatography is, however, a minor concern for ease of process monitoring, and further value to the NG-CSSX technology could be gained by identifying a more lipophilic guanidine analog that is less aqueous soluble and also that lends itself to easier analysis.

The guanidine suppressors under investigation include the active agent of the LIX 79 (DCiTG-L), a commercially available reagent supplied by Cognis (now BASF); DCiTG-E, a reagent synthesized at ORNL; and DCiTG-M1 and DCiTG-M2, two batches of suppressor synthesized using different conditions by Marshallton Research Laboratories. At Oak Ridge National Laboratory (ORNL), a

preparation was developed ${ }^{2}$ based in part on the Cognis patent (now expired) ${ }^{3}$, but employing Exxal-13 as the starting alcohol ${ }^{4}$. Marshallton Research Laboratories subsequently made available their own variant of DCiTG, employing the isotridecyl alcohol feedstock Jarcol ${ }^{\mathrm{TM}} \mathrm{I}-13$. Whereas Exxal-13 has a distribution of molecular weights around a carbon number of 13, Jarcol I-13 has only 13-carbon branched chains, though still with many isomers. 
The study confirms the efficacy of variants of DCiTG from different sources and examines conditions that could potentially impact NG-CSSX solvent performance. Mass-spectral analysis, batch ESS tests and gas chromatography (GC) were used to assess the effect of DCiTG concentration and purity and the nature of the simulant on ESS performance.

\section{EXPERIMENTAL SECTION}

Scrub solution $(0.025 \mathrm{M} \mathrm{NaOH})$ and other $\mathrm{NaOH}$ solutions were prepared by dilution of $1.0 \mathrm{M} \mathrm{NaOH}$ standard solution (Sigma Aldrich). Strip solution $\left(0.010 \mathrm{M} \mathrm{H}_{3} \mathrm{BO}_{3}\right)$ was obtained by dilution of a $0.1 \mathrm{M}$ $\mathrm{H}_{3} \mathrm{BO}_{3}$ stock solution prepared from lab-grade $\mathrm{H}_{3} \mathrm{BO}_{3}$ (min 95.5\%, Sigma Aldrich). Solutions of $\mathrm{HCl}$ were made from 1.0 M HCl standard solution (Baker). Water for preparation of all aqueous solutions was first distilled and then deionized using a Milli- $\mathrm{Q}^{\circledR}$ gradient A10 filtering system equipped with a Quantum $^{\mathrm{TM}}$ Ex Ultrapure Organex Cartridge $\left(18.2 \mathrm{M} \Omega \bullet \mathrm{cm}\right.$ at $25^{\circ} \mathrm{C}$, total organic content $\left.4 \mathrm{ppb}\right)$.

\section{METHODS}

\section{Cesium Distribution Ratios in ESS Tests}

\section{Survey of DCiTS suppressors}

Cesium distribution ratios in ESS tests were determined in a manner similar to that described in Chapter 2 with one extraction stage followed by two scrub stages using $0.025 \mathrm{M} \mathrm{NaOH}$ and three strip stages with $0.010 \mathrm{M} \mathrm{H}_{3} \mathrm{BO}_{3}$. The sequence, abbreviated as $\mathrm{ES}_{2} \mathrm{~S}_{3}$, was run in duplicate for each set of conditions. The organic and aqueous phases were contacted in polypropylene microtubes mounted by clips on a disk rotated at ca. $60 \mathrm{rpm}$ for $60 \mathrm{~min}$ for extractions and $45 \mathrm{~min}$ for scrubs and strips. The solutions were contacted inside an air-box maintained at a temperature of $25 \pm 0.2{ }^{\circ} \mathrm{C}$. After the contacting period, the tubes were centrifuged for $5 \mathrm{~min}$ at $3000 \mathrm{RPM}$ at $25 \pm 0.2^{\circ} \mathrm{C}$. An appropriate aliquot of each phase was subsampled and counted for 5 min using a Packard Cobra II Auto-Gamma counter. A spike of ${ }^{137} \mathrm{Cs}(0.05$ $\mathrm{mCi} / \mathrm{mL}$ stock, $\mathrm{CsCl}$ in $\mathrm{H}_{2} \mathrm{O}$, Eckert \& Ziegler Isotope Products Inc., formally Isotope Products, Burbank, CA) was added to the second and third aqueous strip solutions, owing to the low number of counts remaining after the each strip. To keep samples at the equilibration temperature, tubes were removed individually from the temperature-controlled centrifuge for subsampling. Cesium distribution ratios $\left(D_{\mathrm{Cs}}\right)$ are calculated as the ratio of organic- to aqueous-phase ${ }^{137} \mathrm{Cs}$ activity. 
Based upon the agreement of duplicate samples run within the same set of measurements, the precision of $D_{\mathrm{Cs}}$ values within an ESS experiment run as described has generally been found to worsen in the steps of the sequence as follows: $\pm 5 \%$ (extraction and scrubs), $\pm 10 \%$ (first strip), and $\pm 30 \%$ (second and third strips). This duplicate precision correlates with volumetric precision $( \pm 3 \%)$ and counting precision, which is approximately $\pm 3 \%$ (extraction), $\pm 1 \%$ (scrubs), $\pm 10 \%$ (first strip), and $\pm 30-50 \%$ (second and third strips). Owing to the temperature sensitivity of cesium distribution (ca. $10 \%$ change in $D_{\mathrm{Cs}}$ per ${ }^{\circ} \mathrm{C}^{5}$ ), sample handling can introduce additional error. Thus, effective overall precision of extraction, scrub, and first strip $D_{\mathrm{CS}}$ values is estimated to be on the order of $\pm 10 \%$. Each value presented in the tables in this report is the average of $D_{\mathrm{Cs}}$ values from duplicate ESS runs; the error given represents the standard deviation of the duplicates, the parenthetic number referring to the precision of the corresponding previous digit or, in a few cases, two digits [e.g., 2.11(3) $\times 10^{-1}$ means $0.211 \pm 0.003$ and $3.5(15) \times 10^{-4}$ means $0.00035 \pm 0.00015]$.

\section{Suppressor concentration study}

Standard $\mathrm{ES}_{2} \mathrm{~S}_{3}$ tests were performed according to the procedure described above whilst varying the concentration of the suppressor. This was done in order to ascertain a lower suppressor concentration limit for the NG-CSSX solvent before process failure occurs. NG-CSSX solvents were prepared with DCiTG-M2 suppressor. Concentrations of $0,0.1,0.25,0.5,1,2,3$ and $10 \mathrm{mmol}$ were used in this study and each solution was analyzed within 1 week of preparation.

\section{MASS SPECTROMETRY}

Electrospray (ESI) mass spectrometry was used to investigate the composition of each of the DCiTG guanidines.

\section{PARTITIONING OF DCiTG TO $10 \mathrm{mM}$ BORIC ACID}

Solvent comprised of 0.05 M MaxCalix, 0.5 M Cs-7SB modifier, and 0.003 M DCiTG (DCiTG-M2 from Marshallton) in Isopar ${ }^{\mathrm{TM}} \mathrm{L}$ was used in the following studies. The solvent was prepared as described and prewashed as described in Chapter 2. The NG-CSSX solvents were contacted with $0.010 \mathrm{M}$ boric acid at O:A ratios of 1:10, 1:25, 1:50 and 1:100 in $50 \mathrm{~mL}$ polypropylene tubes sealed with Teflon ${ }^{\circledR}$ tape to avoid organic loss via leakage, mounted by clips on a disk and rotated at ca. $60 \mathrm{rpm}$ for $60 \mathrm{~min}$. The solutions were contacted inside an air-box maintained at a temperature of $25 \pm 0.2{ }^{\circ} \mathrm{C}$. After the contacting period, the tubes were centrifuged for $5 \mathrm{~min}$ at $3000 \mathrm{RPM}$ at $25 \pm 0.2{ }^{\circ} \mathrm{C}$. The organic layers were removed using 
micropipettes and briefly contacted with an equal volume of $1 \mathrm{M} \mathrm{NaOH}$ to ensure the guanidine suppressor was in its neutral form. The solvents were then separated again by centrifugation.

\section{Direct Analysis of DCiTG Concentration Using Gas Chromatography}

Each sample was injected $(1 \mu \mathrm{L})$ into a HP6850 GC-System equipped with hydrogen generator and analyzed using the parameters as discussed in detail in Chapter 5.

\section{RESULTS AND DISCUSSION}

\section{Mass Spectral Analysis of DCiTG Composition}

The ESI mass spectra of DCiTG-based guanidines revealed the differing compositions of the various DCiTG suppressors. The DCiTG-L sample supplied by Cognis and the DCiTG-E synthesized at ORNL using Exxal 13 alcohol feedstock clearly show the varying distribution of the branched $\mathrm{C}_{10}-\mathrm{C}_{15}$ chains in the principal component $[\mathrm{DCiTGH}]^{+}\left(\left[\mathrm{C}_{13}-\mathrm{DCiTGH}\right]^{+}\right.$is found at $\left.\mathrm{m} / \mathrm{z} 406.4\right)$, although $\mathrm{C}_{8}-\mathrm{C}_{14}$ are expected ${ }^{5}$. In all spectra additional peaks are present, identified as trisubstituted alkyl metathesis products together with some di- and monoalkylguanidines.

The identity of the peaks for the various guanidinium components and possible hydrolysis products of DCiTG-M1 and DCiTG-M2 were determined. As expected from the nature of the starting alcohol used by Marshallton, there is no distribution of alkyl carbon number, as species having the isotridecyl group have a single peak. there is a higher percentage of the $N, N^{\prime}, N^{\prime \prime}$-tricyclohexylguanidine (m/z 306.2) and the diand tri-substituted isotridecylguanidine species (506.6 and 606.6, respectively) in the DCiTG-M1 spectrum, suggesting that a higher degree of metathesis has occurred upon synthesis and that the purity of the desired product, $N, N^{\prime}$-dicyclohexyl- $N "$-isotridecylguanidine, is lower.

The observed relative peak intensity for the desired $N, N^{\prime}$-dicyclohexyl- $N "$-isotridecylguanidinium increases from $50 \%$ to $90 \%$ for the DCiTG-M2 suppressor. Although the relative peak intensities are not calibrated, a qualitative assessment can be made that the relative peak intensities can be related to solution concentration and it is clear that the DCiTG-M2 (second batch of DCiTG from Marshallton) is a higherpurity material. 


\section{Extract/Scrub/Strip (ESS) Batch Cesium Distribution Testing}

\section{Reference Limits For Evaluating Ess Performance}

The reference limits for this study were as reported earlier:

$$
\begin{gathered}
D_{\mathrm{Cs}} \geq 40(\mathrm{SRS}) / 5 \text { (Hanford) for extraction, } \\
0.5 \leq D_{\mathrm{Cs}} \leq 10 \text { for scrubbing, } \\
D_{\mathrm{Cs}} \leq 0.1 \text { for stripping. }
\end{gathered}
$$

By contrast with our reference limits, actual process $D_{C s}$ limits corresponding to probable process upset would depend on feed conditions, equipment design, and site requirements and thus have to be set for a given flowsheet. Thus, it should be understood that the above selected limits are reference points that do not necessarily indicate that the performance has deteriorated anywhere near the level of process upset. In fact, multistage centrifugal-contactor flowsheet testing on simulated and real SRS high-level salt waste operated with consistent DF values an order of magnitude above the target performance despite having some scrub and strip stages operating outside of the reference $D_{C s}$ limits given above ${ }^{6}$.

Extraction performance depends strongly on feed composition, particularly the potassium concentration ${ }^{7}$. At the SRS, the bounding limit for potassium is thought to be $0.045 \mathrm{M}^{5}$, which depresses the $D_{C s}$ value noticeably but not severely ${ }^{8}$. With its many stages, the SWPF would be less impacted by a decrease in extraction $D_{\mathrm{Cs}}$ value than the MCU. A value of 40 could be accommodated at the MCU, particularly as it could be increased as needed by dropping the extraction temperature a few degrees ${ }^{8}$. However, at Hanford a wide range of potassium concentrations is found, up to as high as $1 \mathrm{M}$, and we estimate the $D_{\mathrm{Cs}}$ value for NG-CSSX could fall to as low as 3, worst case, based on a previous correlation ${ }^{7}$ adjusted for higher calixarene concentration. Such a low value of $D_{C s}$ can be tolerated for Hanford, because the decontamination requirements for Hanford wastes are generally lower ${ }^{9}$ and because any facility design for Hanford is not constrained by an existing number of contactor stages. A Hanford modular facility could still be designed that employs a few more extraction stages, perhaps compensated by fewer stripping stages $^{7,10}$.

Scrubbing performance can vary somewhat with waste feed, but stripping should perform approximately the same regardless of feed composition in that a properly functioning scrub section should normalize the solvent so that it enters the strip section in approximately the same chemical state. Accordingly, the scrub reference limits $0.5 \leq D_{\mathrm{Cs}} \leq 10$ are not critical in themselves, but rather they indicate potential impact to 
extraction and stripping as follows. If the scrub $D_{\mathrm{Cs}}$ falls much below 0.5 , a significant cesium recycle back to extraction will occur depending on the scrub O:A used (assumed to be at least 3.75:1). If the scrub $D_{\mathrm{Cs}}$ value exceeds 10 , a significant amount of potassium may start to report to the stripping section, impairing its performance.

\section{Survey of DCiTG Performance in ESS Tests}

\section{Performance with SRS-15, SRS-45, and hanford simulants}

Tables 14.1-14.5 compare the $\mathrm{ES}_{2} \mathrm{~S}_{3}$ batch performance of the NG-CSSX solvents prepared with the four different DCiTGs using the three different simulants representing real SRS and Hanford compositions. These compositions are expected to impact extraction mainly through the variation in concentration of potassium, this metal being the primary metal competing with cesium in the waste. Control solvent with no guanidine was run for each simulant, demonstrating in dramatic fashion the inability to strip without a suppressor component. In each case, stripping $D_{C s}$ values exceed the reference limit of 0.1 and tend to increase with successive strips (though not for the Hanford simulant). As a general observation concerning the DCiTG suppressors used at $0.003 \mathrm{M}$, within some experimental scatter all of the DCiTG variants behave similarly for simulants SRS-15, SRS-45, and Hanford.

\section{Cesium distribution ratios for ESS batch tests using different DCiTG based guanidine suppressors with}

\section{SRS-15 simulant ${ }^{a}$}

\begin{tabular}{llllll}
\hline \multicolumn{1}{c}{ Stage } & \multicolumn{1}{c}{ No Suppressor } & \multicolumn{1}{c}{ DCiTG-L } & DCiTG-E & DCiTG-M1 & DCiTG-M2 \\
\hline Extraction & $57.3(26)$ & $51.0(8)$ & $61.0(5)$ & $50.8(5)$ & $55.2(6)$ \\
Scrub 1 & $3.06(6)$ & $2.06(3)$ & $2.8(2)$ & $2.07(3)$ & $2.57(3)$ \\
Scrub 2 & $1.45(7)$ & $8.9(2) \times 10^{-1}$ & $1.01(3)$ & $8.7(3) \times 10^{-1}$ & $1.03(1)$ \\
Strip 1 & $2.11(3) \times 10^{-1}$ & $8.8(4) \times 10^{-4}$ & $1.3(1) \times 10^{-3}$ & $1.09(4) \times 10^{-3}$ & $1.06(1) \times 10^{-3}$ \\
Strip 2 & $6.66(4) \times 10^{-1}$ & $3.2(5) \times 10^{-4}$ & $2.1(8) \times 10^{-4}$ & $5(5) \times 10^{-5}$ & $3.35(5) \times 10^{-4}$ \\
Strip 3 & $1.00(5)$ & $3.5(15) \times 10^{-4}$ & $1.5(2) \times 10^{-4}$ & $1.5(10) \times 10^{-4}$ & $2.8(4) \times 10^{-4}$ \\
\hline
\end{tabular}

${ }^{\mathrm{a}}$ The aqueous phases were SRS-15 waste simulant for extraction, $0.025 \mathrm{M} \mathrm{NaOH}$ for the scrub stages, and $0.01 \mathrm{M}$ H3BO3for the strip stages. Solvent compositions are defined in Table 1 with the DCiTG variant as specified. The O:A ratios were 1:4 for extraction and 3.75:1 for both scrubbing and stripping. Experiments were carried out at a constant temperature of $25 \pm 0.2{ }^{\circ} \mathrm{C}$. 
Cesium distribution ratios for ESS batch tests using different DCiTG based guanidine suppressors with SRS-45 simulant ${ }^{a}$

\begin{tabular}{llllll}
\hline \multicolumn{1}{r}{ Stage } & No Suppressor & \multicolumn{1}{c}{ DCiTG-L } & DCiTG-E & DCiTG-M1 & DCiTG-M2 \\
\hline Extraction & $48(1)$ & $40.7(9)$ & $43(6)$ & $38(2)$ & $42.4(5)$ \\
Scrub 1 & $4.5(11)$ & $2.98(1)$ & $3.46(6)$ & $2.9(1)$ & $3.30(7)$ \\
Scrub 2 & $1.60(2)$ & $1.04(2)$ & $1.6(5)$ & $1.1(1)$ & $1.30(4)$ \\
Strip 1 & $2.43(6) \times 10^{-1}$ & $1.36(9) \times 10^{-3}$ & $1.51(8) \times 10^{-3}$ & $1.6(2) \times 10^{-3}$ & $2.08(2) \times 10^{-3}$ \\
Strip 2 & $5.95(4) \times 10^{-1}$ & $1(1) \times 10^{-4}$ & $3.8(2) \times 10^{-4}$ & $2.9(16) \times 10^{-4}$ & $4.4(8) \times 10^{-4}$ \\
Strip 3 & $8.9(8) \times 10^{-1}$ & $3(1) \times 10^{-4}$ & $2.0(2) \times 10^{-4}$ & $2.6(24) \times 10^{-4}$ & $3.7(5) \times 10^{-4}$ \\
\hline
\end{tabular}

${ }^{\mathrm{a}}$ The aqueous phases were SRS-45 waste simulant for extraction, $0.025 \mathrm{M} \mathrm{NaOH}$ for the scrub stages, and $0.01 \mathrm{M}$ $\mathrm{H}_{3} \mathrm{BO}_{3}$ for the strip stages. Solvent compositions are defined in Table 1 with the DCiTG variant as specified. The O:A ratios were 1:4 for extraction and 3.75:1 for both scrubbing and stripping. Experiments were carried out at a constant temperature of $25 \pm 0.2^{\circ} \mathrm{C}$.

Cesium distribution ratios for ESS batch tests using different DCiTG based guanidine suppressors using hanford simulant ${ }^{a}$

\begin{tabular}{llllll}
\hline \multicolumn{1}{r}{ Stage } & No Suppressor & DCiTG-L & DCiTG-E & DCiTG-M1 & DCiTG-M2 \\
\hline Extraction & $30(1)$ & $25.20(2)$ & $26(2)$ & $26.6(3)$ & $27.4(1)$ \\
Scrub 1 & $4.0(2)$ & $3.45(2)$ & $3.96(2)$ & $3.7(2)$ & $3.7(1)$ \\
Scrub 2 & $1.50(6)$ & $1.07(2)$ & $1.250(4)$ & $1.10(3)$ & $1.00(6)$ \\
Strip 1 & $1.66(7) \times 10^{-1}$ & $4.3(3) \times 10^{-4}$ & $7.7(1) \times 10^{-4}$ & $6.48(6) \times 10^{-4}$ & $6.1(9) \times 10^{-4}$ \\
Strip 2 & $1.39(3) \times 10^{-1}$ & $1.3(3) \times 10^{-5}$ & $2.0(6) \times 10^{-4}$ & $2(3) \times 10^{-4}$ & $2.9(3) \times 10^{-4}$ \\
Strip 3 & $9(3) \times 10^{-2}$ & $2.8(15) \times 10^{-4}$ & $3.0(4) \times 10^{-4}$ & $4(5) \times 10^{-4}$ & $2(1) \times 10^{-4}$ \\
\hline
\end{tabular}

${ }^{a}$ The aqueous phases were Hanford waste simulant for extraction, $0.025 \mathrm{M} \mathrm{NaOH}$ for the scrub stages, and $0.01 \mathrm{M}$ $\mathrm{H}_{3} \mathrm{BO}_{3}$ for the strip stages. Solvent compositions are defined in Table 1 with the DCiTG variant as specified. The O:A ratios were 1:4 for extraction and 3.75:1 for both scrubbing and stripping. Experiments were carried out at a constant temperature of $25 \pm 0.2^{\circ} \mathrm{C}$.

Although the DCiTG variants behaved similarly using the regular waste simulants, addition of surfactant revealed differences in capacity, as shown in Table 6.

Cesium distribution ratios for ESS batch tests using different DCiTG based guanidine suppressors SRSSDS simulant with $5 \times 10^{-4} \mathrm{M}^{a}$

\begin{tabular}{llllll}
\hline \multicolumn{1}{r}{ Stage } & No Suppressor & \multicolumn{1}{c}{ DCiTG-L } & DCiTG-E & DCiTG-M1 & DCiTG-M2 \\
\hline Extraction & $59(1)$ & $63(2)$ & $58(2)$ & $70(2)$ & $60(3)$ \\
Scrub 1 & $4.8(1)$ & $14.2(6)$ & $4.1(6)$ & $30(4)$ & $5.2(6)$ \\
Scrub 2 & $6.10(9)$ & $30(1)$ & $1.9(4)$ & $79(5)$ & $4.0(2)$ \\
Strip 1 & $2.4(2)$ & $6(1) \times 10^{-2}$ & $2.4(5) \times 10^{-3}$ & $17(6)$ & $6(2) \times 10^{-3}$ \\
Strip 2 & $4.6(2)$ & $8.6(20) \times 10^{-3}$ & $3.7(4) \times 10^{-3}$ & $6(5) \times 10^{1}$ & $6.6(4) \times 10^{-4}$ \\
Strip 3 & $7.98(6)$ & $2.1(2) \times 10^{-3}$ & $2.6(6) \times 10^{-3}$ & $4(3) \times 10^{2}$ & $5(1) \times 10^{-4}$ \\
\hline
\end{tabular}

${ }^{a}$ The aqueous phases were SRS-SDS waste simulant for extraction, $0.025 \mathrm{M} \mathrm{NaOH}$ for the scrub stages, and $0.01 \mathrm{M}$ $\mathrm{H}_{3} \mathrm{BO}_{3}$ for the strip stages. Solvent compositions are defined in Table 1 with the DCiTG variant as specified. The O:A ratios were 1:4 for extraction and 3.75:1 for both scrubbing and stripping. Experiments were carried out at a constant temperature of $25 \pm 0.2^{\circ} \mathrm{C}$.

\section{Dependence of ESS Performance on DCiTG Concentration}

The effect of varying the DCiTG-M2 suppressor concentration on the $D_{C s}$ values in the $\mathrm{ES}_{2} \mathrm{~S}_{3}$ sequence is shown in Table 7. From the results, it is apparent that the NG-CSSX solvent can still function effectively 
at suppressor concentrations as low as $2.5 \times 10^{-4} \mathrm{M}$, the stripping reference value of 0.1 not being exceeded until the suppressor concentration reaches $1 \times 10^{-4} \mathrm{M}$. 
Cesium distribution ratios for ESS batch tests with varying concentration of the guanidine suppressor ${ }^{a}$

\begin{tabular}{|c|c|c|c|c|c|c|c|c|}
\hline Stage & $\begin{array}{c}\text { No } \\
\text { Suppressor }^{b}\end{array}$ & $0.0001 \mathrm{M}$ & $0.00025 \mathrm{M}$ & $0.0005 \mathrm{M}$ & $0.001 \mathrm{M}$ & $0.002 \mathrm{M}$ & $0.003 \mathrm{M}$ & $0.01 \mathrm{M}$ \\
\hline Extraction & $66.20(2)$ & $64.2(8)$ & $66.6(15)$ & $64.3(4)$ & $67(1)$ & $69.80(4)$ & $68.5(7)$ & $63.9(6)$ \\
\hline Scrub 1 & $2.80(1)$ & $2.70(3)$ & $2.80(2)$ & $2.80(6)$ & $2.80(1)$ & $2.80(7)$ & $2.80(7)$ & $2.80(2)$ \\
\hline Scrub 2 & $1.3(1)$ & $1.20(4)$ & $1.30(1)$ & $1.20(2)$ & $1.20(1)$ & $1.20(3)$ & $1.20(4)$ & $1.10(2)$ \\
\hline Strip 1 & $2.0(3) \times 10^{-1}$ & $1.15(5) \times 10^{-1}$ & $2.8(4) \times 10^{-2}$ & $7.460(5) \times 10^{-3}$ & $3.3(3) \times 10^{-3}$ & $1.84(8) \times 10^{-3}$ & $1.53(2) \times 10^{-3}$ & $9.9(4) \times 10^{-4}$ \\
\hline Strip 2 & $6.4(2) \times 10^{-1}$ & $3.92(5) \times 10^{-1}$ & $1.24(1) \times 10^{-2}$ & $3.4(5) \times 10^{-4}$ & $2.7(6) \times 10^{-4}$ & $4(3) \times 10^{-4}$ & $5(6) \times 10^{-4}$ & $1.3(8) \times 10^{-4}$ \\
\hline Strip 3 & $1.0(1)$ & $5.0(9) \times 10^{-1}$ & $5.6(9) \times 10^{-3}$ & $3.4(2) \times 10^{-4}$ & $1.6(7) \times 10^{-4}$ & $1.1(5) \times 10^{-4}$ & $1.3(16) \times 10^{-4}$ & $1.62(6) \times 10^{-4}$ \\
\hline
\end{tabular}

${ }^{\mathrm{a}}$ The aqueous phases were SRS-15 waste simulant for extraction, $0.025 \mathrm{M} \mathrm{NaOH}$ for the scrub stages, and $0.01 \mathrm{M} \mathrm{H}_{3} \mathrm{BO}_{3}$ for the strip stages. Solvent composition is defined in Table 1 with DCiTG-M2 as the suppressor. The O:A ratios were 1:4 for extraction and 3.75:1 for both scrubbing and stripping. Experiments were carried out at a constant temperature of $25 \pm 0.2^{\circ} \mathrm{C}$.

${ }^{\mathrm{b}}$ In order to plot a log scale in Figure 6 the suppressor concentration was set at $1 \times 10^{-5} \mathrm{M}$. 


\section{Gas Chromatographic Analysis of DCiTG Partitioning}

Partitioning experiments using $\mathrm{GC}^{11}$, were carried out to quantify the loss of DCiTG to the $0.010 \mathrm{M}$ $\mathrm{H}_{3} \mathrm{BO}_{3}$ strip solution. It is shown in Table 8 that the $P_{\mathrm{GUA}}$ values for DCiTG-M2 are much lower than those of TOA to 0.001 and $0.050 \mathrm{M} \mathrm{HNO}_{3}$, which are on the order of $10^{45}$.

\section{DCiTG-M2 concentration in the NG-CSSX solvent before and after contact with $0.010 \mathrm{M}$ boric acid at different O:A ratios, with corresponding calculated PGUA values}

\begin{tabular}{lcc}
\hline \multicolumn{1}{c}{ O:A Contacts } & [DCiTG-M2] (mM) & P $_{\text {GUA }}{ }^{\mathrm{a}}$ \\
\hline Uncontacted Solvent & $2.36(13)$ & - \\
$\mathrm{O}: \mathrm{A}=1: 10$ & $1.97(4)$ & $40(4)^{b}$ \\
$\mathrm{O}: \mathrm{A}=1: 25$ & $1.33(5)$ & $32(4)$ \\
$\mathrm{O}: \mathrm{A}=1: 50$ & $0.92(9)$ & $32(6)$ \\
$\mathrm{O}: \mathrm{A}=1: 100$ & $0.67(2)$ & $40(3)$ \\
\hline \multicolumn{2}{c}{${ }^{\mathrm{a}}$ The calculated PGUA in each case is the average of 6 analyses, 2 duplicate samples with 3 replicate analyses for } \\
each.
\end{tabular}

\section{CONCLUSIONS AND RECOMMENDATIONS}

Tests of four DCiTG variants from different sources confirmed their efficacy in promoting cesium stripping in standard $\mathrm{ES}_{2} \mathrm{~S}_{3}$ protocol. Extraction $D_{\mathrm{Cs}}$ values decreased with increasing feed concentration of potassium as expected, and good performance overall for the Hanford simulant suggests the feasibility of a modular NG-CSSX unit at Hanford.

Measurements by GC showed that DCiTG-M2 partitions measurably to the aqueous $0.01 \mathrm{M} \mathrm{H}_{3} \mathrm{BO}_{3}$ strip solution. Experimental values of $P_{\mathrm{GUA}}$ vary somewhat with no particular trend, with an average value of $35 \pm 8$. If a lower limit of $1 \mathrm{mM}$ of guanidine is considered a control limit with a comfortable margin before stripping is significantly impaired (i.e., at $<0.25 \mathrm{mM}$ ), then the guanidine will have to be added at intervals of at most 144 solvent cycles to keep the guanidine concentration in the range 1$3 \mathrm{mM}$.

While the ESS results herein have raised no particular issues with regard to differences in the performance of the four DCiTG variants tested with simulated SRS and Hanford waste solutions, the significant partitioning of the DCiTG suppressors to the aqueous strip solution suggests that either or both of the following be considered for improvement of NG-CSSX operation: replace DCiTG with a more lipophilic analog or implement additional process monitoring with regular guanidine makeup. 


\section{APPENDIX C REFERENCES}

1 Duncan, N. C.; Roach, B. D.; Williams, N. J.; Bonnesen, P. V.; Rajbanshi, A.; Moyer, B. A., "N,N',-Dicyclohexyl-N"-Isotridecylguanidine as Suppressor for the Next Generation Caustic Side Solvent Extraction (NG-CSSX) Process." Separation Science and Technology, 47, (January 2012), 2074-2087.

2 Bonnesen, P. V.; Duncan, N. C.; Rajbanshi, A. "Preparation of 1,3-dicyclohexyl-2(isotridecyl)guanidine and 1,3-dicyclohexyl-2-(isotridecyl)guanidine hydrochloride (DCiTG and DCiTG.HCl, suppressor for Next Generation CSSX process solvent)," ORNL-LTRNGCSSX-007, Oak Ridge National Laboratory, Oak Ridge, TN, April 2011.

3 Lin, L.-T. W.; Mattison, P. L.; Virnig, M. J. "Recovery of Precious Metal." United States Patent 5,028,259, July 2, 1991 (Filed March 6, 1991).

4 ExxonMobil Chemical-“Exxal ${ }^{\circledR} 13$ Tridecyl Alcohol Sales Specification Rev. 3", http://www.exxonmobilchemical.com/Chem-English/brands/exxalalcohols.aspx? $\ln =$ productsservices, 2008.

5 Moyer, B. A.; Alexandratos, S. D.; Bonnesen, P. V.; Brown, G. M.; Caton, Jr., J. E.; Delmau, L. H.; Duchemin, C. R.; Haverlock, T. J.; Levitskaia, T. G.; Maskarinec, M. P.; Sloop, Jr., F. V.; Stine, C. L. "Caustic-Side Solvent Extraction Chemical and Physical Properties: Progress in FY 2000 and FY 2001.” ORNL/TM-2001/285, Oak Ridge National Laboratory, Oak Ridge, TN, 2002.

6 Pierce, R. A.; Peters, T. B.; Crowder, M. L.; Caldwell, T. D.; Pak, D. J.; Fink, S. D.; Blessing, R. W.; Washington, A. L. "Demonstration of the Next-Generation Caustic Side Solvent Extraction Solvent with 2-cm Centrifugal Contactors using Tank 49H Waste and Waste Simulant," SRNL-STI-2011-00589. Savannah River National Laboratory, Aiken, SC, September 2011.

7 Delmau, L. H.; Birdwell, Jr., J. F.; McFarlane, J.; Moyer, B. A.; "Robustness of the CSSX Process to Feed Variation: Efficient Cesium Removal from the High Potassium Wastes at Hanford." Solvent Extr. Ion Exch., 28, 19-48, 2010.

8 Moyer, B. A.; Bonnesen, P. V.; Delmau, L. H.; Sloop, Jr., F. V.; Williams, N. J.; Birdwell, Jr., J. F.; Lee, D. L.; Leonard, R. A.; Fink, S. D.; Peters, T. B.; Geeting, M. W. "Development of the Next-Generation Caustic-Side Solvent Extraction (NG-CSSX) Process for Cesium Removal from High-Level Tank Waste." Paper 11346, Proc. Waste Management 2011, February 27-March 3, 2011, Phoenix, AZ; Waste Management Symposia, Tempe, AZ.

9 May, T. H.; Pajunen, A.; Baker, J.; Himes, M.; Stegen, G. "Project W-551 Interim Pretreatment System Pre-conceptual Candidate Technology Descriptions," RPP-RPT-37551, Rev. 1. CH2MHILL Hanford Group (for the U.S. Department of Energy), Richland, WA (2008).

10 Moyer, B. A.; Birdwell, Jr., J. F.; Delmau, L. H.; McFarlane, J. "Caustic-Side Solvent Extraction Modeling for Hanford Interim Waste Pretreatment System," ORNL/TM-2008/073. Oak Ridge National Laboratory, Oak Ridge, TN, 2008.

11 Duncan, N. C.; Bonnesen, P. V. "Method Development for the Gas Chromatography (GC) Analysis of 1,3-Dicyclohexyl-2-(isotridecyl)guanidine (DCITG, Suppressor for Next Generation CSSX Process Solvent)," ORNL-LTR-NGCSSX-011, Oak Ridge National Laboratory, Oak Ridge, TN, April 2011. 



\section{REFERENCES}

1 "Tank Waste Research and Development Plan," United States Department of Energy Office of Environmental Research, Office of Technology Innovation and Development, Washington, DC, 2010.

2 "Report to Congress: Status of Environmental Management Initiatives to Accelerate the Reduction of Environmental Risks and Challenges Posed by the Legacy of the Cold War." DOE/EM-0001, U.S. Department of Energy, Office of Environmental Management, Washington, DC, 2009.

${ }^{3}$ Moyer, B. A.; Bonnesen, P. V.; Delmau, L. H.; Sloop, Jr., F. V.; Williams, N. J.; Birdwell, Jr., J. F.; Lee, D. L.; Leonard, R. A.; Fink, S. D.; Peters, T. B.; Geeting, M. W. "Development of the NextGeneration Caustic-Side Solvent Extraction (NG-CSSX) Process for Cesium Removal from High Level Tank Waste.” Paper 11346, Proc. Waste Management 2011, February 27-March 3, 2011, Phoenix, AZ; Waste Management Symposia, Tempe, AZ.

${ }^{4}$ Leonard, R. "Initial Flowsheet for the Next Generation CSSX Process in the MCU. Report for 2010, ANL LTR-NGCSSX-001. Argonne National Laboratory, Illinois, 2010.

${ }^{5}$ Moyer, B. A.; Delmau, L. H.; Duncan, N. C.; Ensor, D. D.; Hill, T. G.; Lee, D. L.; Roach, B. D.; Sloop, Jr., F. V.; Williams, N. J. "Recommended DCiTG Guanidine Suppressor for the Next-Generation Caustic-Side Solvent Extraction Process," ORNL/TM-2012/625. Oak Ridge National Laboratory, Oak Ridge, TN. 2013.

6 Roach, B. D.; Williams, N. J.; Duncan, N. C.; Moyer, B. A. "N,N'-Dicyclohexyl-N"Isotridecylguanidine Supressor Variants for the Next Generation Caustic Side Solvent Extraction (NG-CSSX) Process," ORNL LTR-NGCSSX-018. Oak Ridge National Laboratory, Oak Ridge, TN, 2011.

${ }^{7}$ Moyer, B. A.; Alexandratos, S. D.; Bonnesen, P. V.; Brown, G. M.; Caton, Jr., J. E.; Delmau, L. H.; Duchemin, C. R.; Haverlock, T. J.; Levitskaia, T. G.; Maskarinec, M. P.; Sloop, Jr., F. V.; Stine, C. L. "Caustic-Side Solvent Extraction Chemical and Physical Properties: Progress in FY 2000 and FY 2001." ORNL/TM-2001/285, Oak Ridge National Laboratory, Oak Ridge, TN, 2002.

${ }^{8}$ Moyer, B. A.; Birdwell, Jr., J. F.; Delmau, L. H.; McFarlane, J. "Caustic-Side Solvent Extraction Modeling for Hanford Interim Waste Pretreatment System," ORNL/TM-2008/073. Oak Ridge National Laboratory, Oak Ridge, TN, 2008.

${ }^{9}$ Peterson, R. A. "Preparation of Simulated Waste Solutions for Solvent Extraction Testing." WSRC-RP2000-00361, Westinghouse Savannah River Company, Aiken, SC, 2000.

${ }^{10}$ Delmau, L. H.; Birdwell, Jr., J. F.; McFarlane, J.; Moyer, B. A.; "Robustness of the CSSX Process to Feed Variation: Efficient Cesium Removal from the High Potassium Wastes at Hanford." Solvent Extr. Ion Exch. 2010, 28, 19-48.

${ }^{11}$ Delmau, L. H., Haverlock, T. J.; Bazelaire, E.; Bonnesen, P. V.; Ditto, M. E.; Moyer, B. A. "Alternatives to Nitric Acid Stripping in the Caustic-Side Solvent Extraction (CSSX) Process." Solvent Extr. Ion Exch. 2009, 27, 172-198.

${ }^{12}$ Lamarsh, J.; Baratta, A. "Introduction to Nuclear Engineering," $3^{\text {rd }}$ ed.; Prentice Hall, Upper Saddle River, 2001.

${ }^{13}$ Peterman, D. R.; Meikrantz, D. H.; Law, J. D.; Riddle, C. L.; Todd, T. A.; Greenhalgh, M. R.; Tillotson, R. D.; Bartsch, R. A.; Moyer, B. A.; Delmau, L. H.; Bonnesen, P. V. Extractant Compositions for Co-extracting Cesium and Strontium, A Method of Separating Cesium and Strontium from an Aqueous Feed, Calixarene Compounds, and An Alcohol Modifier. US Patent No. 8158088, Apr. 17, 2012.

${ }^{14}$ Delmau, L. H.; Van Berkle, G. J.; Bonnesen, P. V. ; Moyer, B. A. "Improved Performance of the Alkaline-Side CSEX Process for Cesium Extraction from Alkaline High Level Waste Obtained by 
Characterization of the Effect of Surfactant Impurities," ORNL/TM-1999/209, Oak Ridge National Laboratory, Oak Ridge, TN, October 1999.

${ }^{15}$ Koeckelberghs, G.; De Cremer, L.; Vanormelingen, W.; Dehaen, W.; Verbiest, T.; Persoons, A.; Samyn, C. "Improved synthesis of N-alkyl substituted dithienyl[3,2-b:2',3'-d]pyrroles." Tetrahedron, 2005, 61, 687-691.

${ }^{16}$ Lin, L.-T. W.; Mattison, P. L.; Virnig, M. J. "Recovery of Precious Metal." United States Patent 5,028,259, July 2, 1991 (Filed March 6, 1991).

${ }^{17}$ Mitsunobu, O. "The Use of Diethyl Azidodicarboxylate and Triphenylphosphine in Synthesis and Transformation of Natural Products." Synthesis, 1981, 1-28 (review).

${ }^{18}$ Baes, C. F., "Modeling solvent extraction systems with SXFIT Source", Solvent Extr. Ion Exch. 2001, 19(2), 193-213.

${ }^{19}$ Delmau, L. H.; Bostick, D. A.; Haverlock, T. J.; Moyer, B. A. "Caustic-Side Solvent Extraction: Extended Equilibrium Modeling of Cesium and Potassium Distribution Behavior," Report ORNL/TM-2002/116. Oak Ridge National Laboratory, Oak Ridge, TN, May 2002.

${ }^{20}$ Baes, C. F., Jr. "SXLSQI: A Program for Modeling Solvent Extraction Systems," Report ORNL/TM13604. Oak Ridge National Laboratory, Oak Ridge, TN, December 1998.

${ }^{21}$ Duncan, N. C.; Roach, B. D.; Williams, N. J.; Bonnesen, P. V.; Rajbanshi, A.; Moyer, B. A., "N,N',Dicyclohexyl-N"-Isotridecylguanidine as Suppressor for the Next Generation Caustic Side Solvent Extraction (NG-CSSX) Process." Sep. Sci. Technol. 2012, 47, 2074-2087.

${ }^{22}$ Harris, D. C. Quantitative Chemical Analysis. 8th ed. W.H. Freeman and Company: New York, 2010; Chapter 5.

${ }^{23}$ Delmau, L. H.; Haverlock, T. J.; Sloop, Jr., F. V.; Moyer, B. A. "Caustic-Side Solvent Extraction: Prediction of Cesium Extraction from Actual Wastes and Actual Waste Simulants," ORNL Report ORNL/TM-2003/011. Oak Ridge National Laboratory, Oak Ridge, TN, 2003.

${ }^{24}$ Peters, T. B.; Fink, S. D. "Results of the First Extraction-Scrub-Strip Testing Using Improved Solvent Formulations and Actual Savannah River Site Waste," SRNL-STI-2010-00586. Savannah River National Laboratory, Aiken, SC, 2010.

25 Peters, T. B.; Poirier, M. R.; Nash, C. A.; Fondeur, F. F.; Fink, S. D.; Brown, S. J.; Brass, E. A. "Testing and Startup of the Savannah River Site Integrated Salt Disposition Process," Paper 9193. Proc. Waste Management 2009 (WM '09), Phoenix, AZ, Mar. 1-5, 2009, Waste Management Symposia, Tempe, AZ (2009).

${ }^{26}$ Bonnesen, P. V.; Delmau, L. H.; Haverlock, T. J.; Moyer, B. A. "Alkaline-Side Extraction of Cesium from Savannah River Tank Waste Using a Calixarene-Crown Ether Extractant." ORNL/TM-13704, Oak Ridge National Laboratory, Oak Ridge, TN, 1998.

${ }^{27}$ Birdwell, Jr., J. F.; Anderson, K. K. "Evaluation of 5-cm Centrifugal Contactor Hydraulic and Mass Transfer Performance for Caustic-Side Solvent Extraction of Cesium." ORNL/TM-2001/137, Oak Ridge National Laboratory, Oak Ridge, TN, 2001.

${ }^{28}$ Birdwell, Jr., J. F.; Anderson, K. K. "Evaluation of Mass Transfer Performance for Caustic-Side Solvent Extraction of Cesium in a Conventional 5-cm Centrifugal Contactor." ORNL/TM2001/278, Oak Ridge National Laboratory, Oak Ridge, TN, January 2002.

${ }^{29}$ Law, J. D.; Tillotson, R. D.; Todd, T. A. "Evaluation of the Hydraulic Performance and Mass Transfer Efficiency of the CSSX Process with the Optimized Solvent in a Single stage of 5.5-Cm Diameter Centrifugal Contactor." INEEL/EXT-02-01109, Idaho National Engineering and Environmental Laboratory, Idaho Falls, ID, September 2002.

${ }^{30}$ Lentsch, R. D.; Stephens, A. B.; Bartling, K. E.; Singer, S. A. "Caustic-Side Solvent Extraction FullScale Test." Paper 8431, Proc. Waste Management 2008, Feb. 24-28, 2008, Phoenix, AZ; Waste Management Symposia, Tempe, AZ.

${ }^{31}$ Brown, S. J. "Caustic Side Solvent Extraction at the Savannah River Site: Operating Experience and 
Lessons Learned." Paper 10105, Proc. Waste Management 2010, Phoenix, AZ, Mar. 7-11, 2010; Waste Management Symposia, Tempe, AZ.

${ }^{32}$ Moyer, B. A.; Birdwell, Jr., J. F.; Bonnesen, P. V.; Delmau, L. H. "Use of Macrocycles in NuclearWaste Cleanup: A Real-World Application of a Calixcrown in Technology for the Separation of Cesium." In Macrocyclic Chemistry-Current Trends and Future, K. Gloe, Ed., Springer, Dordrecht, pp. 383-405 (2005).

${ }^{33}$ Delmau, L. H.; Birdwell, Jr., J. F.; Bonnesen, P. V.; Foote, L. J.; Haverlock, T. J.; Klatt, L. N.; Lee, D. D.; Leonard, R. A.; Levitskaia, T. G.; Maskarinec, M. P.; Moyer, B. A.; Sloop, Jr., F. V.; Tomkins, B. A. "Caustic-Side Solvent Extraction: Chemical and Physical Properties of the Optimized Solvent." ORNL/TM-2002/190, Oak Ridge National Laboratory, Oak Ridge, TN (2002).

${ }^{34}$ Sachleben, R. A.; Bonnesen, P. V.; Descezeaud, T.; Haverlock, T. J.; Urvoas, A.; Moyer, B. A. "Surveying the Extraction of Cesium Nitrate by 1,3-Alternate Calix[4]arene Crown-6 Ethers in 1,2Dichloroethane." Solvent Extr. Ion Exch. 1999, 17, 1445-1459.

${ }^{35}$ Shevchenko, V. B.; Smelov, V. S. J. Nucl. Energy. Part A. Reactor Science 1960, 11, 196.

${ }^{36}$ Viswanadhan, V. N.; Ghose, A. K.; Revankar, G. R.; Robins, R. K. J. Chem. Inf. Comput. Sci. 1989, 29, 163.

${ }^{37}$ Kerr, G. D.; Eckerman, K. F.; "Radiation Dosimetry for the CSSX Process," ORNL letter report to K. J. Rueter, Westinghouse Savannah River Company, October 12, 2000.

${ }^{38}$ Lamarsh, J.; Baratta, A. "Introduction to Nuclear Engineering," $3^{\text {rd }}$ ed.; Prentice Hall, Upper Saddle River, 2001.

${ }^{39}$ Olszanski, A.; Klassen, N.; Ross, C.; Shortt, K. "The IRS Fricke Dosimetry System. Ionizing Radiation Standards," PIRS-0815. Institute for National Measurement Standards, Ottawa, Canada, August 2002.

${ }^{40}$ Standard Practice for Using the Fricke Reference-Standard Dosimetry System. ASTM Standard E 1026-04.

${ }^{41}$ Geeting, M. W.; Savannah River Remediation Co., personal communication to B. A. Moyer, September $16,2011$.

${ }^{42}$ Aguayo, S.; Valenzuela, J. L.; Parga, J. R.; Lewis, R. G.; Cruz, M. "Continuous Laboratory Gold Solvent Extraction from Cyanide Solutions using LIX ${ }^{\circledR} 79$ Reagent." Chem. Eng. Technol. 2007, 30(11), 1532-1536.

${ }^{43}$ Delmau, L. H.; Moyer, B. A. "Solvent Blending Strategy to Upgrade MCU CSSX Solvent to Equivalent Next-Generation CSSX Solvent," ORNL LTR-NGCSSX-010, Oak Ridge National Laboratory, Oak Ridge, TN, July 22, 2011.

${ }^{44}$ Pierce, R. A.; Peters, T. B.; Crowder, M. L.; Caldwell, T. D.; Pak, D. J.; Fink, S. D.; Blessing, R. W.; Washington, A. L. "Demonstration of the Next-Generation Caustic Side Solvent Extraction Solvent with 2-cm Centrifugal Contactors using Tank 49H Waste and Waste Simulant," SRNL-STI-201100589. Savannah River National Laboratory, Aiken, SC, September 2011. 